JOURNAL OF THE

AMERICAN MATHEMATICAL SOCIETY

Volume 10, Number 4, October 1997, Pages 865-937

S 0894-0347(97)00243-9

\title{
DETERMINANT OF PERIOD INTEGRALS
}

\author{
TAKESHI SAITO AND TOMOHIDE TERASOMA
}

\section{INTRODUCTION}

We prove a formula for the determinant of period integrals. Period integrals arise from comparison between Betti cohomologies and de Rham cohomologies. Our formula Theorem 1 in Section 4 expresses the determinant of period integrals as the product of the periods evaluated at the relative canonical cycles and special values of the $\Gamma$-function. The formula is a Hodge version of Theorem 1 of [S2] for $\ell$-adic cohomology. Together with this, it gives a motivic formula, Theorem 2 in Section 5. It particularly implies that the category of motives of rank 1 associated to an algebraic Hecke character is closed under taking the determinant of cohomology. Hence it gives a support to a conjecture of Deligne, Conjecture 8.1 iii [D4]; a motive of rank 1 is associated to an algebraic Hecke character. A typical example of our formula is that the period of a Fermat hypersurface is a product of special values of the $\Gamma$-function (cf. proof of Lemma 5.4). The main theorem for $X=\mathbb{P}^{1}$ is a reformulation of a theorem of the second named author [T], Theorem 1.2. The theorem is proved by reducing to this case by induction on dimension using a Lefschetz pencil.

We describe the result. Let $U$ be a smooth quasi-projective variety over a subfield $k$ of $\mathbb{C}$ and $F$ another subfield of $\mathbb{C}$. The determinant of the periods, the main subject of the paper, is defined for a triple $\mathcal{M}=((\mathcal{E}, \nabla), V, \rho)$ consisting of the following three data:

(1) $\mathcal{E}$ is a locally free sheaf on $U$ with an integrable connection $\nabla$ regular singular along the boundary. It gives rise to a local system

$$
\operatorname{Ker}\left(\nabla^{a n}\right)=\operatorname{Ker}\left(\nabla^{a n}: \mathcal{E}^{a n} \rightarrow\left(\mathcal{E} \otimes \Omega_{U}^{1}\right)^{a n}\right) .
$$

(2) $V$ is a local system of $F$-vector spaces on $U^{a n}$.

(3) $\rho$ is an isomorphism between local systems of $\mathbb{C}$-vector spaces

$$
\rho: V \otimes_{F} \mathbb{C} \rightarrow \operatorname{Ker}\left(\nabla^{a n}\right) .
$$

The category of such triples is denoted by $M_{k, F}(U)$. We consider the compact support cohomology of the data (1) and (2). For the datum (1), we define the de Rham cohomology with compact support $H_{c}^{q}(U, D R(\mathcal{E}, \nabla))$ by taking a small extension to a compactification $X$ of $U$ such that the complement $D$ is a divisor with normal crossings. The precise definitions will be given in Section 1. For the

Received by the editors June 3, 1996 and, in revised form, May 7, 1997.

1991 Mathematics Subject Classification. Primary 14F99.

(C)1997 American Mathematical Society 
data (2), $H_{c}^{q}\left(U^{a n}, V\right)$ denotes the singular cohomology with compact support. The comparison theorem gives us an isomorphism:

$$
H_{c}^{q}(\rho): H_{c}^{q}\left(U^{a n}, V\right) \otimes_{F} \mathbb{C} \rightarrow H_{c}^{q}(U, D R(\mathcal{E}, \nabla)) \otimes_{k} \mathbb{C} .
$$

Note that the cohomologies $H_{c}^{q}\left(U^{a n}, V\right)$ and $H_{c}^{q}(U, D R(\mathcal{E}, \nabla))$ are vector spaces over $F$ and $k$ respectively. The determinant of the periods is defined as the alternating product

$$
\operatorname{per}_{c}(\mathcal{M})=\prod_{q} \operatorname{det} P_{q}^{(-1)^{q+1}}
$$

where $P_{q}=\left(p_{i j}^{q}\right)$ is the matrix representing the $\mathbb{C}$-linear map $H_{c}^{q}(\rho)$ for a basis $\left(v_{i}^{q}\right)_{i}$ of $H_{c}^{q}\left(U^{a n}, V\right)$ over $F$ and a basis $\left(e_{j}^{q}\right)_{j}$ of $H_{c}^{i}(U, D R(\mathcal{E}, \nabla))$ over $k: \rho\left(v_{i}^{q}\right)=$ $\sum_{k} p_{j i}^{q} e_{j}$. It is well-defined as an element in $k^{\times} \backslash \mathbb{C}^{\times} / F^{\times}$.

Theorem 1. (in vague form) Under the notation as above,

$$
\operatorname{per}_{c}(\mathcal{M}) \operatorname{per}_{c}(\mathbf{1})^{-\operatorname{rank} \mathcal{M}}=\Gamma(\nabla: \mathcal{M})^{-1} \times\left(\operatorname{det} \mathcal{M}, c_{X} \bmod D\right)
$$

in $k^{\times} \backslash \mathbb{C}^{\times} / F^{\times}$.

The precise statement is given in Section 4. In the right hand side, the symbol $X$ means a compactification of $U$ having a divisor $D$ with simple normal crossing as the complement. The $\Gamma$-factor $\Gamma(\nabla: \mathcal{M})$ is a suitable product of the values of the $\Gamma$-function at the eigenvalues of the residue of an extension of the connection $\nabla$ along components of divisor $D$, which will be defined at the end of Section 1. The relative canonical cycle $c_{X \bmod D}$ is the refined top Chern class of the locally free sheaf $\Omega_{X}^{1}(\log D)$ with respect to the residues res ${ }_{i}$ : $\Omega_{X}^{1}(\log D) \otimes \mathcal{O}_{D_{i}} \rightarrow \mathcal{O}_{D_{i}}$ defined in Section 2. From the point of view of RiemannRoch, the reason why the canonical class appears in the formula is the equality $\sum(-1)^{q} \operatorname{ch}\left(\Omega_{X}^{q}(\log D)\right)=(-1)^{n} c_{n}\left(\Omega_{X}(\log D)\right)$. (See the Remark in Section 6.) The pairing $\left(\operatorname{det} \mathcal{M}, c_{X} \bmod D\right)$ is defined as the tame symbol which will be defined in Section 3.

We give a motivic formula Theorem 2 in Section 5 . Note that Theorem 1 gives the determinant of periods for virtual objects in $M_{k, F}(U)$ of rank 0 . To obtain a formula in general, it is enough to consider this for the unit object $\mathbf{1}$. If $D=\emptyset$, $\operatorname{per}_{c}(\mathbf{1})^{2}=(2 \pi i)^{\operatorname{dim} X \chi_{c}(X)}$ by Poincaré duality and $\operatorname{per}_{c}(\mathbf{1})^{2}$ is a multiple of $2 \pi i$ in general. For the $\ell$-adic setting, a similar formula is given in [S2].

Let us explain the contents of each section. In Section 1, we review generalities on integrable connections and local systems. Using the comparison theorem for the de Rham cohomology and the singular cohomology, we define the determinant of periods $\operatorname{per}_{c}(\mathcal{M})$. At the end of the section, the $\Gamma$-factor $\Gamma(\nabla: \mathcal{M})$ is defined.

Next, in Section 2, we define the relative Chow group $C H^{n}(X \bmod D)$ as $H^{n}\left(X, \mathcal{K}_{n}(X \bmod D)\right)$, where $\mathcal{K}_{n}$ stands for the sheaf of Quillen's $K$-groups, $\mathcal{K}_{n}(X \bmod D)$ is the complex $\mathcal{K}_{n}(X) \rightarrow \bigoplus_{i} \mathcal{K}_{n}\left(D_{i}\right)$, and the $D_{i}$ 's are the components of the divisor $D$. We give the definition of the top Chern classes of a locally free $\mathcal{O}_{X}$-module trivialized on $D$, and give the basic properties. This is quite similar to [S1]. The main result in the section is an adelic description of $C H^{n}(X \bmod D)$ as the cokernel of the complex $\bigoplus_{y \in X_{1}} B_{y} \rightarrow \bigoplus_{x \in X_{0}} A_{x}$. Here $A_{x}$ is an extension of $\mathbb{Z}$ by $\bigoplus_{i \in I_{x}} \kappa(x)^{\times}$( $I_{x}$ is the set of $i$ 's such that $x$ belongs to $D_{i}$ and $\kappa(x)$ is the residue field), $B_{y}$ is defined in terms of $K_{2}$ and the boundary 
map is defined in terms of tame symbols. Its proof makes heavy use of techniques in K-theory and will be given in the Appendix.

Section 3 is devoted to the construction of the pairing:

$$
M P i c_{k, F}(U) \times C H^{n}(X \bmod D) \rightarrow M P i c_{k, F}(k)=k^{\times} \backslash \mathbb{C}^{\times} / F^{\times}
$$

where $M P i c_{k, F}(U)$ denotes the group of isomorphism classes of rank one objects in $M_{k, F}(U)$. By the description of $C H^{n}(X \bmod D)$, given in Section 2, it is enough to define the local pairing $M P i c_{k, F}(U) \times A_{x} \rightarrow M P i c_{k, F}(k)$ and prove a reciprocity law, the annihilation of the pairing $M P i c_{k, F}(U) \times B_{y} \rightarrow M P i c_{k, F}(k)$. For a point $x \in U$, the pairing $(\mathcal{M}, x)$ with the class $[x]$ is the norm $\left.N_{x / k} \mathcal{M}\right|_{x}$ of the fiber of $\mathcal{M}$ at $x$. We prove the reciprocity law in two steps. First we prove it for curves. Using the norm map, it is reduced to the case for $\mathbb{P}^{1}$. To prove it in higher dimension, we define and use a generalization of the tame symbol. We reduce it to the case for curves. Section 3 is closed with a remark on the philosophical background for the definition of tame symbol.

The precise statement and the proof of the main theorem are given in Section 4 . First we prove it for $\mathbb{P}^{1}$. This is a slight modification of the result in $[\mathrm{T}]$. We prove the general case by induction on dimension using a Lefschetz pencil $X \rightarrow Y=\mathbb{P}^{1}$. We compute the residue of the Gauss-Manin connection on the relative de Rham cohomology. This computation is local on $Y$. Then we apply a formula for Chern classes for the fibration and the induction proceeds. At the end of the section, a variant of the main result, which will be used in the next section, is presented.

In Section 5, we first give a reformulation Theorem $\mathrm{S}$ of Theorem 1 [S2] on the determinant of $\ell$-adic cohomology using the relative Chow group. To state and prove it, an etale version of the tame symbol is defined and studied. It is a partial generalization to higher dimension of the integration of torsor in [D3]. The main result in Section 5 is a motivic version, Theorem 2. The Jacobi sum à la Anderson appears in the formulation of the theorem. We introduce the notion of being "determinantally motivic" and prove it is stable under taking the determinant of the cohomology as a corollary.

In Section 6 we give a refinement Theorem 3 of Theorem 1 for curves. Theorem 1 only means an equality of the isomorphism classes but Theorem 2 provides an isomorphism in terms of Deligne's Riemann-Roch [D7].

This is a full version of [ST].

Remark. We have changed the definition of the determinant of the period in [ST] to its inverse, in order to make it compatible with [D4], 8.7. Because of this change, the sign in Theorem loc.cit. and Corollary of Theorem 1 in Section 4 looks apparently inconsistent. We apologize for the inconvenience.

\section{NOTATION}

Section 1. $\left(\mathcal{E}_{X}, \nabla\right)$ (logarithmic integrable connection) $\ldots . . . \quad 1$ (a)

$D R\left(\mathcal{E}_{X}\right)$ (logarithmic de Rham complex) ............ 1 (a)

$\operatorname{res}_{i} \nabla$ (residue of a logarithmic connection) $\ldots \ldots \ldots \ldots \ldots$.

$\Phi_{\mathcal{E}_{X}, i}(T)$ (eigenpolynomial of the residue) $\ldots \ldots \ldots \ldots \ldots$.

$H_{c}^{q}(U, D R(\mathcal{E}))$ (de Rham cohomology with compact support) $\ldots . \quad 1$ (a)

$T_{i}$ (monodromy operator) $\ldots \ldots \ldots \ldots \ldots \ldots \ldots \ldots . \ldots \ldots$

$M_{k_{0}, F}(U)$ (category of triples) $\ldots \ldots \ldots \ldots \ldots \ldots \ldots . \ldots \ldots \ldots$

$\operatorname{det} \mathcal{M}$ (determinant) $\ldots \ldots \ldots \ldots \ldots \ldots \ldots \ldots$ 
$P_{k_{0}, F}$ (category of triples of rank 1) $\ldots \ldots \ldots \ldots \ldots \ldots . .1(\mathrm{~b})$

$M P i c_{k_{0}, F}$ (isomorphism class group of $\left.P_{k_{0}, F}\right) \ldots \ldots \ldots \ldots .1$ (b)

$\operatorname{per}(\mathcal{M}), \operatorname{per}_{c}(\mathcal{M})$ (period, period with compact support) $\ldots \ldots . \quad 1$ (b)

$\Gamma(\nabla: \mathcal{M})$ (Gamma-factor) ................. 1 (c)

$\operatorname{det}^{\Gamma} R \Gamma_{c}(U, \mathcal{M})$ (determinant modified by Gamma-factor) $\ldots . . \quad 1$ (c)

Section 2. $C H^{n}(X \bmod D)($ relative Chow group) $\ldots \ldots \ldots . \quad 2$ (a)

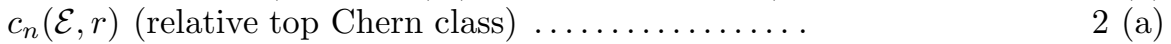

$c_{X \bmod D}$ (relative canonical class) $\ldots \ldots \ldots \ldots \ldots \ldots . .2$ (a)

$X_{i}$ (set of points of dimension $i$ ) $\ldots \ldots \ldots \ldots \ldots \ldots . \quad 2$ (b)

$A(L)$ (group of tensors of $L) \ldots \ldots \ldots \ldots \ldots \ldots . .2(\mathrm{~b})$

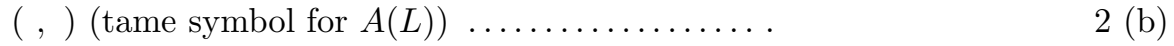

$A_{x}$ (extension of $\mathbb{Z}$ by direct sum of $\left.\kappa(x)^{\times}\right) \ldots \ldots \ldots \ldots .2(\mathrm{~b})$

$B_{y}$ (extension of $\kappa(y)^{\times}$by direct sum of $\left.K_{2}(y)\right) \ldots \ldots \ldots \ldots .2(\mathrm{~b})$

$\partial_{x, y}$ ( boundary map) $\ldots \ldots \ldots \ldots \ldots \ldots \ldots \ldots . .2(\mathrm{~b})$

Section 3. ( , ) (tame symbol) .............. 3 (a)

$(,)_{x}$ (tame symbol) $\ldots \ldots \ldots \ldots \ldots \ldots \ldots \ldots$.

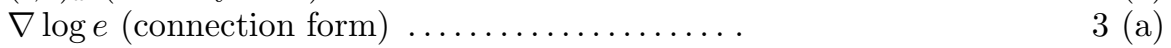

$(\nabla, f)^{\sim}$ (connection defining tame symbol) $\ldots \ldots \ldots \ldots . .3($ a)

$(V, f)^{\sim}$ (local system defining tame symbol) $\ldots . \ldots \ldots \ldots . .3$ (a)

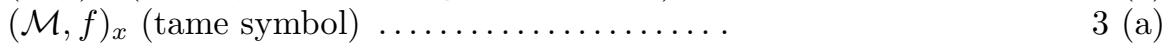

$(\varphi, f)^{\sim}$ (holomorphic function defining tame symbol) $\ldots . . . . \quad 3$ (a)

$(\varphi, f)_{x}$ (tame symbol) $\ldots \ldots \ldots \ldots \ldots \ldots \ldots \ldots . . . \ldots \ldots$

$N_{L / k}, N_{x / k}$ (norm) $\ldots \ldots \ldots \ldots \ldots \ldots \ldots \ldots \ldots$

$N_{X / Y}($ norm $) \ldots \ldots \ldots \ldots \ldots \ldots \ldots \ldots \ldots . .3(\mathrm{~b})$

$(,)_{i}$ (tame symbol along a boundary component) $\ldots \ldots \ldots . .3$ (c)

Section 4. $\quad M_{k_{0}, F}^{\prime}(U), M \operatorname{Pic}_{k_{0}, F}^{\prime}, \operatorname{per}_{c, F}(\mathcal{M}), \operatorname{per}_{c, F}^{\Gamma}(\mathcal{M})$ (variants) . 4 (f) $\left(\mathcal{E}^{(F)}, \nabla^{(F)}\right)$ (connection on $\left.U \otimes_{k_{0}} F\right) \ldots \ldots \ldots \ldots \ldots$.

Section 5. $\quad \pi_{1}(k)^{\mathrm{ab}}, \pi_{1}(X)^{\mathrm{ab}}, \pi_{1}(U)^{\mathrm{ab}, \text { tame }}$ (abelian fundamental group) ...................... 5 (a)

$B_{F}(k)^{0}, \mathbb{B}^{0}$ (group of data to define Jacobi sums) $\ldots \ldots \ldots \ldots . \quad 5$ (b)

$J(\mathbf{a})($ Jacobi sum) $\ldots \ldots \ldots \ldots \ldots \ldots \ldots \ldots \ldots$

$J_{D, V_{\lambda}}$ (Jacobi sum defined by ramification) $\ldots \ldots \ldots \ldots \ldots .5$ (b)

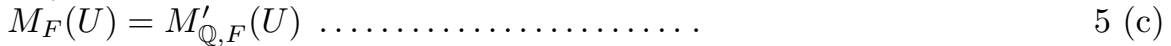

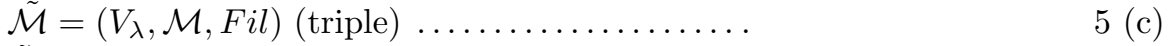

$\tilde{J}(\mathbf{a})$ (triple defined by Jacobi sum) $\ldots \ldots \ldots \ldots \ldots \ldots . .5$ (c)

$\operatorname{det}_{F} R \Gamma_{c}(U, \tilde{\mathcal{M}}),\left(\operatorname{det}_{F} \tilde{\mathcal{M}}, c\right), J_{D, \tilde{\mathcal{M}}} \ldots \ldots \ldots \ldots \ldots \ldots$

Section 6. $\quad(,(\mathcal{L}, r))$ (tame symbol) $\ldots \ldots \ldots \ldots \ldots \ldots .6$ (a)

$\left(\operatorname{det} \mathcal{M}, \mathbf{c}_{X \bmod D}\right)$ (pairing with relative canonical class) $\ldots \ldots .6$ (a)

$(\mathcal{M}, f)$ (tame symbol) $\ldots . \ldots \ldots \ldots \ldots \ldots \ldots .6$ (a)

$D R R$ (isomorphism of Deligne-Riemann-Roch) ......... 6 (a)

\section{Connections and Local Systems}

(a) Integrable connections with regular singularities. Let $k$ be a field of characteristic 0 and $U$ be a smooth separated scheme over $k$. An integrable connection $\nabla$ on a locally free $\mathcal{O}_{U}$-module $\mathcal{E}$ of finite rank is an additive morphism 
$\nabla: \mathcal{E} \rightarrow \mathcal{E} \otimes \Omega_{U}^{1}$ satisfying $\nabla(f e)=f \nabla e+e \otimes d f$ for local sections of $f$ of $\mathcal{O}_{U}$ and $e$ of $\mathcal{E}$ and $\nabla^{2}=0: \mathcal{E} \rightarrow \mathcal{E} \otimes \Omega_{U}^{2}$. Let $X$ be a smooth separated scheme over $k$ of dimension $n$ including $U$ as the complement of a divisor $D$ with simple normal crossings. A divisor $D$ is said to have simple normal crossings if its irreducible components $D_{i, i \in I}$ are smooth and their intersections $D_{J}=\bigcap_{i \in J} D_{i}$ for $J \subset I$ are transversal. Let $\Omega_{X}^{q}(\log D)$ be the sheaf of differential $q$-forms with logarithmic poles along $D$. For a coherent $\mathcal{O}_{X}$-module $\mathcal{E}_{X}$, we call a logarithmic integrable connection an additive morphism $\nabla: \mathcal{E}_{X} \rightarrow \mathcal{E}_{X} \otimes \Omega_{X}^{1}(\log D)$ satisfying $\nabla(f e)=f \nabla e+e \otimes d f$ for local sections of $f$ of $\mathcal{O}_{X}$ and $e$ of $\mathcal{E}_{X}$ and $\nabla^{2}=0$. We call a logarithmic integrable connection $\left(\mathcal{E}_{X}, \nabla\right)$ an extension of an integrable connection $(\mathcal{E}, \nabla)$ on $U$ to $X$ when an isomorphism $\left.\left(\mathcal{E}_{X}, \nabla\right)\right|_{U} \stackrel{\sim}{\rightarrow}(\mathcal{E}, \nabla)$ is given.

Lemma 1.1. For an integrable connection $(\mathcal{E}, \nabla)$ on $U$, the following conditions (1)-(3) are equivalent.

(1) There exists an extension $\left(\mathcal{E}_{X}, \nabla\right)$ to $X$.

(2) There exists an extension $\left(\mathcal{E}_{X}, \nabla\right)$ to $X$ such that $\mathcal{E}_{X}$ is reflexive.

(3) For the generic point $\eta_{i}$ of each irreducible component $D_{i}$ of $D$, there exists a free $\mathcal{O}_{X, \eta_{i}}$-module $\mathcal{E}_{i}$ of finite rank and a logarithmic integrable connection $\nabla: \mathcal{E}_{i} \rightarrow \mathcal{E}_{i} \otimes \Omega_{X}^{1}(\log D)_{\eta_{i}}$ extending the generic fiber $\nabla: \mathcal{E}_{\xi} \rightarrow \mathcal{E}_{\xi} \otimes \Omega_{X, \xi}^{1}$ at the generic point $\xi$ of $X$.

Proof. It is clear that (2) implies (1) and (3). On the other hand, each of (1) and (3) implies that there exist an open subscheme $U^{\prime}$ of $X$ including $U$ such that the codimension of $X-U^{\prime} \geq 2$ and a locally free extension $\mathcal{E}_{U^{\prime}}$ of $\mathcal{E}$ to $U^{\prime}$. If $j: U^{\prime} \rightarrow X$ denotes the open immersion, $\mathcal{E}_{X}=j_{*} \mathcal{E}_{U^{\prime}}$ is a reflexive extension of $\mathcal{E}$ to $X$.

We say an integrable connection $(\mathcal{E}, \nabla)$ on $U$ has regular singularity along $D$ if the equivalent conditions in Lemma 1.1 are satisfied.

Corollary. Let $X$ and $X^{\prime}$ be proper smooth schemes over $k$ including $U$ as the complement of divisors $D$ and $D^{\prime}$ with simple normal crossings respectively. An integrable connection $(\mathcal{E}, \nabla)$ on $U$ has regular singularity along $D$ if and only if it also does along $D^{\prime}$.

Proof. We may assume there is a proper morphism $X^{\prime} \rightarrow X$ inducing the identity on $U$. Then by (1), regular singularity along $D$ implies that along $D^{\prime}$. By (3) we have the inverse implication.

We say an integrable connection $(\mathcal{E}, \nabla)$ on $U$ has regular singularity along the boundary, if there is one (hence for any) proper $X$ including $U$ as the complement of a divisor $D$ with simple normal crossings such that it has regular singularity along $D$. The integrable connections on $U$ with regular singularity along the boundary form a neutral Tannakian category.

We define the de Rham cohomology with compact support $H_{c}^{q}(U, D R(\mathcal{E}))$ for an integrable connection $(\mathcal{E}, \nabla)$ with regular singularity along the boundary. In the following, we always consider reflexive extensions unless stated otherwise. Let $\left(\mathcal{E}_{X}, \nabla\right)$ be a reflexive logarithmic integrable connection on $(X, D)$. The de Rham complex

$$
\begin{gathered}
D R\left(\mathcal{E}_{X}\right)=\left(\mathcal{E}_{X} \otimes \Omega_{X}^{\bullet}(\log D)\right) \\
=\left[\mathcal{E}_{X} \stackrel{\nabla}{\rightarrow} \mathcal{E}_{X} \otimes \Omega_{X}^{1}(\log D) \stackrel{\nabla}{\rightarrow} \mathcal{E}_{X} \otimes \Omega_{X}^{2}(\log D) \rightarrow \cdots\right]
\end{gathered}
$$


is defined as usual. For each component $D_{i}$ of $D$, the residue $\operatorname{res}_{i} \nabla \in \operatorname{End}_{\mathcal{O}_{D_{i}}}\left(\left.\mathcal{E}_{X}\right|_{D_{i}}\right)$ of $\nabla$ at $D_{i}$ is defined as the map induced by

$$
\mathcal{E}_{X} \stackrel{\nabla}{\rightarrow} \mathcal{E}_{X} \otimes \Omega_{X}^{1}(\log D) \stackrel{i d \otimes \text { res }_{i}}{\longrightarrow} \mathcal{E}_{X} \otimes_{\mathcal{O}_{X}} \mathcal{O}_{D_{i}} .
$$

We define the characteristic polynomial $\Phi_{\mathcal{E}_{X, i}}(T)=\operatorname{det}\left(T-\operatorname{res}_{i} \nabla\right) \in \kappa\left(\eta_{i}\right)[T]$. It is in $k_{i}[T]$ where $k_{i}$ is the constant field of $D_{i}$ by [D1], Chapitre II, Proposition 3.10 (ii).

Definition 1. Let $(\mathcal{E}, \nabla)$ be an integrable connection on $U$ with regular singularity along the boundary and let $X$ be a proper smooth $k$-scheme including $U$ as the complement of a divisor $D$ with simple normal crossings.

(1) An extension $\left(\mathcal{E}_{X}, \nabla\right)$ of $(\mathcal{E}, \nabla)$ is said to be small (resp. big) at a component $D_{i}$ of $D$ if any integer $n \leq 0$ (resp. $n \geq 1$ ) is not a root of the polynomial $\Phi_{\mathcal{E}_{X}, i}(T)=0$. We say $\left(\mathcal{E}_{X}, \nabla\right)$ is small if it is small at every irreducible component $D_{i}$.

(2) We define the compact support cohomology

$$
H_{c}^{q}(U, D R(\mathcal{E}))
$$

to be $H^{q}\left(X, D R\left(\mathcal{E}_{X}\right)\right)$ for a small extension $\left(\mathcal{E}_{X}, \nabla\right)$ of the small extension of $(\mathcal{E}, \nabla)$ to $X$.

The extension $\left(\mathcal{O}_{X}(-D), d\right)$ of $\left(\mathcal{O}_{U}, d\right)$ is small since $\Phi_{i}(T)=T-1$. For an integrable connection $(\mathcal{E}, \nabla)$ on $U$ with regular singularity along the boundary, there exists a small extension (resp. big extension) of $(\mathcal{E}, \nabla)$ to $X$ and they form a cofinal system since $\Phi_{\mathcal{E}_{X}\left(\sum n_{j} D_{j}\right), i}(T)=\Phi_{\mathcal{E}_{X}, i}\left(T+n_{i}\right)$. The definition (2) of the compact support cohomology is justified by the following Lemma 1.2. By Riemann-Roch, the Euler number $\chi_{c}(U, D R(\mathcal{E}))=\sum_{p}(-1)^{p} \operatorname{dim}_{k} H_{c}^{p}(U, D R(\mathcal{E}))$ is rank $\mathcal{E} \times \chi_{c}(U)$, where $\chi_{c}(U)$ is the Euler number $\chi_{c}\left(U, D R\left(\mathcal{O}_{U}\right)\right)$.

Lemma 1.2. Let $(\mathcal{E}, \nabla)$ be an integrable connection on $U$ with regular singularity along the boundary and let $X$ be a proper smooth $k$-scheme including $U$ as the complement of a divisor $D$ with simple normal crossings.

(1) For small (resp. big) extensions $\mathcal{E}_{X} \subset \mathcal{E}_{X}^{\prime}$ of $(\mathcal{E}, \nabla)$, the inclusion of the de Rham complexes $D R\left(\mathcal{E}_{X}\right) \subset D R\left(\mathcal{E}_{X}^{\prime}\right)$ is a quasi-isomorphism.

(2) For a small extension $\mathcal{E}_{X}$, there is a canonical perfect pairing

$$
H^{p}\left(X, D R\left(\mathcal{E}_{X}\right)\right) \otimes H^{q}\left(U, D R\left(\mathcal{E}^{*}\right)\right) \rightarrow k,
$$

$$
\text { for } p+q=2 n \text {. }
$$

Proof. (1) We give a proof for the small case and the big case is done in the same way. There is a sequence of extensions $\mathcal{E}_{X}=\mathcal{E}_{0} \subset \mathcal{E}_{1} \subset \cdots \subset \mathcal{E}_{\ell} \subset \cdots \subset \mathcal{E}_{m}=\mathcal{E}_{X}^{\prime}$ of extensions of $(\mathcal{E}, \nabla)$ such that $\mathcal{E}_{\ell} / \mathcal{E}_{\ell-1}$ is an $\mathcal{O}_{D_{i(\ell)}}$-module for $1 \leq \ell \leq m$. In fact, a reflexive extension $\mathcal{E}_{X} \subset \mathcal{E}_{X}^{\prime \prime} \subset \mathcal{E}_{X}^{\prime}$ is determined by a family of sub $\mathcal{O}_{X, \eta_{i}}$-modules $\mathcal{E}_{X, i} \subset \mathcal{E}_{X, i}^{\prime \prime} \subset \mathcal{E}_{X, i}^{\prime}$ such that $\nabla\left(\mathcal{E}_{X, i}^{\prime \prime}\right) \subset \mathcal{E}_{X, i}^{\prime \prime} \otimes \Omega_{X}^{1}(\log D)$ for each irreducible component $D_{i}$ (cf. proof of Lemma 1.1).

By induction, we may assume that there exists an irreducible component $D_{i}$ such that the quotient $\mathcal{E}_{X}^{\prime} / \mathcal{E}_{X}$ is an $\mathcal{O}_{D_{i}}$-module. We show that the quotient $D R\left(\mathcal{E}_{X}^{\prime}\right) / D R\left(\mathcal{E}_{X}\right)=\left(\mathcal{E}_{X}^{\prime} /\left.\mathcal{E}_{X} \otimes_{\mathcal{O}_{D_{i}}} \Omega_{X}^{\bullet}(\log D)\right|_{D_{i}}\right)$ is acyclic. We define a decreasing 
filtration $F$ on $D R\left(\mathcal{E}_{X}^{\prime}\right) / D R\left(\mathcal{E}_{X}\right)$ by

$$
F^{p}\left(D R\left(\mathcal{E}_{X}^{\prime}\right) / D R\left(\mathcal{E}_{X}\right)\right)^{q}= \begin{cases}0, & q<p, \\ \mathcal{E}_{X}^{\prime} / \mathcal{E}_{X} \otimes_{\mathcal{O}_{D_{i}}} \Omega_{D_{i}}^{q}\left(\left.\log D\right|_{D_{i}}\right), & q=p, \\ \mathcal{E}_{X}^{\prime} /\left.\mathcal{E}_{X} \otimes_{\mathcal{O}_{D_{i}}} \Omega_{X}^{q}(\log D)\right|_{D_{i}}, & q>p .\end{cases}
$$

Here $\left.D\right|_{D_{i}}=\bigcup_{j \neq i} D_{j} \cap D_{i}$. The $g r$-complex $G r_{F}^{p}$ is given by

$$
\operatorname{res}_{i} \nabla \otimes i d: \mathcal{E}_{X}^{\prime} / \mathcal{E}_{X} \otimes_{\mathcal{O}_{D_{i}}} \Omega_{D_{i}}^{p}\left(\left.\log D\right|_{D_{i}}\right) \rightarrow \mathcal{E}_{X}^{\prime} / \mathcal{E}_{X} \otimes_{\mathcal{O}_{D_{i}}} \Omega_{D_{i}}^{p}\left(\left.\log D\right|_{D_{i}}\right) .
$$

By the assumption that $\mathcal{E}_{X}$ is small, it is enough to show the following lemma.

Lemma 1.3. Let $\left(\mathcal{E}_{X}, \nabla\right)$ be a reflexive extension. If $\operatorname{res}_{i} \nabla \in \operatorname{End}_{\mathcal{O}_{D_{i}}}\left(\left.\mathcal{E}_{X}\right|_{D_{i}}\right)$ is an isomorphism at $\eta_{i}$, it is an isomorphism on $D_{i}$.

Proof. We use the following elementary fact.

Lemma 1.4. Let $\mathcal{E}$ be a reflexive $\mathcal{O}_{X}$-module on a regular scheme $X$. Then the complement of the open subscheme $W$ of $X$ where $\mathcal{E}$ is locally free is of codimension $\geq 3$.

Proof. See [O], Ch. 2, Lemma 1.1.10.

Let $j: W \cap D_{i} \rightarrow D_{i}$ be the open immersion. Since $\mathcal{E}_{X}$ is reflexive, we see that $\left.\mathcal{E}_{X}\right|_{D_{i}}$ is a submodule of the reflexive sheaf $j_{*} j^{*}\left(\left.\mathcal{E}_{X}\right|_{D_{i}}\right)$. By the assumption, $\operatorname{det} \operatorname{res}_{i} \nabla$ is a non-zero constant on $W \cap D_{i}$. Hence $\operatorname{res}_{i} \nabla$ induces an automorphism of $j_{*} j^{*}\left(\left.\mathcal{E}_{X}\right|_{D_{i}}\right)$. Now the assertion is a consequence of the following elementary fact.

Lemma 1.5. Let $f$ be an automorphism of a coherent $\mathcal{O}_{X}$-module $\mathcal{M}$ on a noetherian scheme $X$ and $\mathcal{N} \subset \mathcal{M}$ be a sub-coherent $\mathcal{O}_{X}$-module stable under $f$. Then the restriction $\left.f\right|_{\mathcal{N}}$ is an automorphism.

Proof. We have an increasing sequence of submodules $\mathcal{N} \subset f^{-1}(\mathcal{N}) \subset \cdots$. Hence we have $f^{-n}(\mathcal{N})=f^{-n-1}(\mathcal{N})$ for some $n$ and $f(\mathcal{N})=\mathcal{N}$.

(2) The desired pairing is induced from the pairing

$$
D R\left(\mathcal{E}_{X}\right) \otimes D R\left(\mathcal{E}_{X}^{*}\right) \rightarrow \Omega_{X}^{n}[-n] .
$$

We prove its perfectness by a transcendental argument.

Let $k_{0}$ be a subfield of the complex number field $\mathbb{C}$. We assume $k$ is a finite extension of $k_{0}$. Let $X, U$ and $D$ over $k$ be as above. Let $X^{a n}$ be the complex manifold with underlying set $X(\mathbb{C})=\operatorname{Hom}_{k_{0}-S c h}(\operatorname{Spec}(\mathbb{C}), X)$. An integrable connection $(\mathcal{E}, \nabla)$ on $U$ defines an analytic integrable connection $\left(\mathcal{E}^{a n}, \nabla^{a n}: \mathcal{E}^{a n} \rightarrow\right.$ $\left.\mathcal{E}^{a n} \otimes \Omega_{U}^{1}{ }^{a n}\right)$ on $U^{a n}$. The sheaf Ker $\nabla^{a n}$ of flat sections is a local system of $\mathbb{C}$ vector spaces on $U^{a n}$. The canonical map $\operatorname{Ker} \nabla^{a n} \rightarrow D R\left(\mathcal{E}^{a n}\right)=\left(\mathcal{E}^{a n} \otimes \Omega_{U}^{\bullet a n}\right)$ is a quasi-isomorphism by Poincaré's lemma.

Lemma 1.6. A reflexive extension $\mathcal{E}_{X}$ is small if and only if the analytic de Rham complex $D R\left(\mathcal{E}_{X}\right)^{\text {an }}$ is quasi-isomorphic to $j_{!}^{a n} D R(\mathcal{E})^{\text {an }}$.

Proof. We may assume $k=\mathbb{C}$. We may further assume $\mathcal{E}_{X}$ is small and locally free by (1) and [D1], p. 94, Prop. 5.4. In this case, we can show that the natural morphism $j_{!} D R(\mathcal{E})^{a n} \rightarrow D R\left(\mathcal{E}_{X}\right)^{\text {an }}$ is a quasi-isomorphism by a similar argument in [D1], p. 102, Prop. 6.8.

We show that, if $\mathcal{E}_{X}$ is not small, the cohomology sheaf $\left.\mathcal{H}^{0}\left(D R\left(\mathcal{E}_{X}\right)^{a n}\right)\right|_{D^{a n}} \neq 0$. Take a maximal small extension $\mathcal{E}_{X}^{\prime} \subset \mathcal{E}_{X}$. By replacing $\mathcal{E}_{X}$ by $\mathcal{E}_{X} \cap \mathcal{E}_{X}^{\prime}\left(D_{i}\right)$ and 
shrinking $X$ if necessary, we may assume that $\mathcal{E}_{X}$ and $\mathcal{E}_{X}^{\prime}$ are free, $D=D_{i}$ is irreducible and that $\mathcal{F}=\mathcal{E}_{X} / \mathcal{E}_{X}^{\prime}$ is a free $\mathcal{O}_{D}$-module. By the maximality of $\mathcal{E}_{X}^{\prime}$, the characteristic polynomial $\operatorname{det}(T-\operatorname{res} \nabla, \mathcal{F}) \in k_{i}[T]$ has 0 as a solution. We show that the coherent sheaf $\mathcal{E}_{X}^{\prime \prime}=\operatorname{Ker}\left(\operatorname{res} \nabla: \mathcal{E}_{X} \rightarrow \mathcal{F}\right)$ is an extension of $(\mathcal{E}, \nabla)$. The composition $\mathcal{E}_{X} \otimes \Omega_{X}^{1}(\log D) \stackrel{\nabla}{\rightarrow} \mathcal{E}_{X} \otimes \Omega_{X}^{2}(\log D) \stackrel{\text { res }}{\rightarrow} \mathcal{E}_{X} / \mathcal{E}_{X}^{\prime} \otimes \Omega_{D}^{1}$ is given by res $\nabla \otimes i d$. Hence on $\mathcal{E}_{X}^{\prime \prime}$, we have $(\operatorname{res} \nabla \otimes i d) \circ \nabla=\operatorname{res} \circ \nabla^{2}=0$. This means $\mathcal{E}_{X}^{\prime \prime}$ is an extension. Replacing $\mathcal{E}_{X}$ by $\mathcal{E}_{X}^{\prime \prime}$, we may further assume that res $\nabla$ is 0 on $\mathcal{F}=\mathcal{E}_{X} / \mathcal{E}_{X}^{\prime}$. The logarithmic connection $\nabla$ on $\mathcal{E}_{X}$ induces an integrable connection $\nabla: \mathcal{F} \rightarrow \mathcal{F} \otimes \Omega_{D}^{1}$. Then we have exact sequences of complexes

$$
\begin{gathered}
0 \rightarrow D R_{X}\left(\mathcal{E}_{X}^{\prime}\right) \rightarrow D R_{X}\left(\mathcal{E}_{X}\right) \rightarrow \mathcal{F} \otimes \Omega_{X}^{\bullet}(\log D) \rightarrow 0, \\
0 \rightarrow D R_{D}(\mathcal{F}) \rightarrow \mathcal{F} \otimes \Omega_{X}^{\bullet}(\log D) \rightarrow D R_{D}(\mathcal{F})[-1] \rightarrow 0 .
\end{gathered}
$$

By the assumption that $\mathcal{E}_{X}^{\prime}$ is small we obtain

$$
\mathcal{H}^{q}\left(D R_{X}\left(\mathcal{E}_{X}\right)^{a n}\right) \simeq \begin{cases}\operatorname{Ker} \nabla^{a n} \text { on } \mathcal{F}^{a n}, & q=0,1, \\ 0 & \text { otherwise. }\end{cases}
$$

Thus Lemma 1.6 is proved.

We return to the proof of (2). We assume $k=\mathbb{C}$. Since $\mathcal{E}_{X}$ is small, we have

$$
H^{p}\left(X, D R\left(\mathcal{E}_{X}\right)\right) \simeq H^{p}\left(X^{a n}, D R\left(\mathcal{E}_{X}\right)^{a n}\right) \simeq H^{p}\left(X^{a n}, j_{!} \operatorname{Ker}\left(\nabla^{a n}\right)\right)
$$

by (1). By [D1], p. 98, Théorème 6.2,

$$
H^{q}\left(U, D R\left(\mathcal{E}^{*}\right)\right) \simeq H^{q}\left(U^{a n}, \operatorname{Ker}\left(\nabla^{*, a n}\right)\right) .
$$

The assertion is reduced to the Poincaré duality.

The local system $\operatorname{Ker} \nabla^{a n}$ defines a monodromy representation of the fundamental group $\pi_{1}\left(U^{a n}, \bar{x}\right) \rightarrow \operatorname{Aut}(\mathcal{E}(\bar{x}))$ for $\bar{x} \in U^{a n}$. For an open subscheme $D_{i}^{\prime}$ of an irreducible component $D_{i}$ of $D$ where $\left.\mathcal{E}_{X}\right|_{D_{i}^{\prime}}$ is locally free, let $T_{i} \in \operatorname{Aut}\left(\left.\mathcal{E}_{X}^{a n}\right|_{D_{i}^{\prime a n}}\right)$ be the limit of the positive generator of the monodromy along $D_{i}^{a n}$. Then we have

$$
T_{i}=\exp \left(-2 \pi \sqrt{-1} \operatorname{res}_{i} \nabla^{a n}\right)
$$

by [D1], Chapitre II, Proposition 3.11.

(b) Definition of period. Let $F$ be another subfield of $\mathbb{C}$.

Definition 2. For a smooth separated scheme $U$ over $k_{0}$, we define $M_{k_{0}, F}(U)$ to be the category consisting of triples $\mathcal{M}=((\mathcal{E}, \nabla), V, \rho)$ :

(1) A locally free $\mathcal{O}_{U}$-module $\mathcal{E}$ of finite rank with an integrable connection $\nabla$ : $\mathcal{E} \rightarrow \mathcal{E} \otimes \Omega_{U}^{1}$ which has regular singularity along the boundary.

(2) A local system $V$ of $F$-vector spaces on the complex manifold $U^{a n}$.

(3) A morphism $\rho: V \rightarrow \mathcal{E}^{a n}$ on $U^{a n}$ inducing an isomorphism $V \otimes_{F} \mathbb{C} \stackrel{\sim}{\rightarrow} \operatorname{Ker} \nabla^{a n}$ of local systems of $\mathbb{C}$-vector spaces on $U^{\text {an }}$.

We call $(\mathcal{E}, \nabla)$ the de Rham component, $V$ the Betti component and $\rho$ the comparison isomorphism of a triple $\mathcal{M}$. For an object $\mathcal{M}=((\mathcal{E}, \nabla), V, \rho)$ of $M_{k_{0}, F}(U)$, we call $\operatorname{rank}_{\mathcal{O}_{X}} \mathcal{E}_{X}=\operatorname{rank}_{F} V$ the rank of $\mathcal{M}$ and define the determinant $\operatorname{det} \mathcal{M}$ to be the triple $\left(\left(\operatorname{det}_{\mathcal{O}_{X}} \mathcal{E}\right.\right.$, trace $\left.\nabla\right)$, $\operatorname{det} V$, $\left.\operatorname{det} \rho\right)$. Let $M P i c_{k_{0}, F}(U)$ be the group of the isomorphism classes with respect to the tensor product of the subcategory $P_{k_{0}, F}(U)$ of $M_{k_{0}, F}(U)$ consisting of the rank 1 objects and the isomorphisms. The unit element is the class of $\mathbf{1}=\left(\left(\mathcal{O}_{U}, d\right), F\right.$, canonical map). For a finite extension $k$ of $k_{0}$, the class group $M P i c_{k_{0}, F}(\operatorname{Spec} k)$ is canonically isomorphic to 
$k^{\times} \backslash\left(k \otimes_{k_{0}} \mathbb{C}\right)^{\times} /\left(F^{\times}\right)^{\text {Hom }_{k_{0}}(k, \mathbb{C})}$. For an object $\mathcal{M}=(\mathcal{E}, V, \rho)$ of $P_{k_{0}, F}(k)$ with bases $e$ of $\mathcal{E}$ over $k$ and $v$ of $V$ over $F$, its isomorphism class [M] is given by the class of $e / \rho(v)$. Here we identify $k \otimes \mathbb{C} \simeq \mathbb{C}^{\mathrm{Hom}_{k_{0}}(k, \mathbb{C})}$ by $x \otimes y \mapsto(\sigma(x) y)_{\sigma}$.

For a smooth separated scheme $U$ over a finite extension $k$ of $k_{0}$ and $\mathcal{M}=$ $((\mathcal{E}, \nabla), V, \rho) \in M_{k_{0}, F}(U)$, we define the determinant of periods

$$
\operatorname{per}(\mathcal{M}), \operatorname{per}_{c}(\mathcal{M}) \in k^{\times} \backslash\left(k \otimes_{k_{0}} \mathbb{C}\right)^{\times} /\left(F^{\times}\right)^{\operatorname{Hom}_{k_{0}}(k, \mathbb{C})}
$$

as the classes $[\operatorname{det} R \Gamma(U, \mathcal{M})]$ and $\left[\operatorname{det} R \Gamma_{c}(U, \mathcal{M})\right] \in M P i c_{k_{0}, F}(\operatorname{Spec} k)$ defined below. Let $X$ be a proper smooth scheme over $k$ including $U$ as the complement of a divisor $D$ with simple normal crossings. For an extension $\left(\mathcal{E}_{X}, \nabla\right)$ of $(\mathcal{E}, \nabla)$ to $X$, we have an isomorphism $H^{q}\left(X, D R\left(\mathcal{E}_{X}\right)\right) \otimes_{k_{0}} \mathbb{C} \simeq H^{q}\left(X^{a n}, D R\left(\mathcal{E}_{X}\right)^{a n}\right)$ by GAGA. The isomorphism $\rho$ induces an isomorphism $H^{q}\left(X^{a n}, D R\left(\mathcal{E}_{X}\right)^{a n}\right) \simeq H_{c}^{q}\left(U^{a n}, V\right) \otimes_{F} \mathbb{C}$ (resp. $H^{q}\left(U^{a n}, V\right) \otimes_{F} \mathbb{C}$ ) if $\mathcal{E}_{X}$ is small (resp. big) by Lemma 1.6. Hence we obtain canonical isomorphisms $H_{c}^{q}(\rho): H_{c}^{q}(U, D R(\mathcal{E})) \otimes_{k} \mathbb{C} \simeq H_{c}^{q}\left(U^{a n}, V\right) \otimes_{F} \mathbb{C}$ and $H^{q}(\rho): H^{q}(U, D R(\mathcal{E})) \otimes_{k} \mathbb{C} \simeq H^{q}\left(U^{a n}, V\right) \otimes_{F} \mathbb{C}$. The triples

$$
\begin{aligned}
& H_{c}^{q}(U, \mathcal{M})=\left(H_{c}^{q}(U, D R(\mathcal{E})), H_{c}^{q}\left(U^{a n}, V\right), H_{c}^{q}(\rho)\right), \\
& H^{q}(U, \mathcal{M})=\left(H^{q}(U, D R(\mathcal{E})), H^{q}\left(U^{a n}, V\right), H^{q}(\rho)\right)
\end{aligned}
$$

are objects of $M_{k_{0}, F}(\operatorname{Spec} k)$.

Definition 3. For a triple $\mathcal{M} \in M_{k_{0}, F}(U)$, the determinants of the periods

$$
\operatorname{per}_{c}(\mathcal{M})=\left[\operatorname{det} R \Gamma_{c}(U, \mathcal{M})\right], \quad \operatorname{per}(\mathcal{M})=[\operatorname{det} R \Gamma(U, \mathcal{M})]
$$

$\in M P i c_{k_{0}, F}=k^{\times} \backslash\left(k \otimes_{k_{0}} \mathbb{C}\right)^{\times} /\left(F^{\times}\right)^{\text {Hom }_{k_{0}}(k, \mathbb{C})}$ with or without compact support are defined as the isomorphism classes of the alternating tensor product of the determinant,

$$
\begin{aligned}
\operatorname{det} R \Gamma_{c}(U, \mathcal{M}) & =\otimes_{q}\left(\operatorname{det} H_{c}^{q}(U, \mathcal{M})\right)^{\otimes(-1)^{q}}, \\
\operatorname{det} R \Gamma(U, \mathcal{M}) & =\otimes_{q}\left(\operatorname{det} H^{q}(U, \mathcal{M})\right)^{\otimes(-1)^{q}}
\end{aligned}
$$

$\in P_{k_{0}, F}(k)$.

More concretely, if $k=k_{0}$, and, for each $q$, if $e^{q}$ is a basis of the $k$-vector space $H_{c}^{q}(U, D R(\mathcal{E})), v^{q}$ is a basis of the $F$-vector space $H^{q}\left(U^{a n}, V\right)$ and if $P^{q}$ is the matrix representing $H_{c}^{q}(\rho)$ with respect to the bases $e^{q}$ and $v^{q}$, then $\operatorname{per}_{c}(\mathcal{M})=$ $\prod_{q}\left(\operatorname{det} P^{q}\right)^{(-1)^{q+1}} \in k^{\times} \backslash \mathbb{C}^{\times} / F^{\times}$. Our main theorem (Theorem 1 in Section 4 ) is a formula for the period $\operatorname{per}_{c}(\mathcal{M}) \cdot \operatorname{per}_{c}(\mathbf{1})^{-\operatorname{rank} \mathcal{M}}$.

By Serre duality and Poincaré duality, the canonical pairing

$$
H_{c}^{p}(U, \mathcal{M}) \times H^{q}\left(U, \mathcal{M}^{*}\right) \rightarrow \mathbf{1}(-\operatorname{dim} X)
$$

is perfect for $p+q=2 \operatorname{dim} X$. Hence we obtain

Lemma 1.7. The canonical pairing

$$
\operatorname{det} R \Gamma_{c}(U, \mathcal{M}) \times \operatorname{det} R \Gamma\left(U, \mathcal{M}^{*}\right) \rightarrow \mathbf{1}\left(-\operatorname{dim} X \cdot \chi_{c}(U, \mathcal{M})\right)
$$

is perfect. The equality

$$
\operatorname{per}_{c}(\mathcal{M}) \cdot \operatorname{per}_{c}(\mathbf{1})^{-\operatorname{rank} \mathcal{M}}=\left(\operatorname{per}\left(\mathcal{M}^{*}\right) \cdot \operatorname{per}(\mathbf{1})^{-\operatorname{rank} \mathcal{M}}\right)^{-1}
$$

holds. 
(c) $\Gamma$-factor. We define a variant $\operatorname{det}^{\Gamma} R \Gamma_{c}(U, \mathcal{M})$ of $\operatorname{det} R \Gamma_{c}(U, \mathcal{M})$ as the product with the $\Gamma$-factor $\Gamma(\nabla: \mathcal{M})$ defined as follows.

Let $k[T]^{\prime}$ denote the monoid of non-zero polynomials such that the coefficient of the highest degree is 1 and that it is not divisible by $(T-n)$ for any non-positive integers $n \leq 0$. For such a polynomial $f \in k[T]^{\prime}$, we define

$$
\Gamma(f) \in\left(k \otimes_{k_{0}} \mathbb{C}\right)^{\times}=\left(\mathbb{C}^{\times}\right)^{\text {Hom }_{k_{0}}(k, \mathbb{C})}
$$

as $\left(\prod_{i} \Gamma\left(\alpha_{\sigma, i}\right)\right)_{\sigma}$ where $\sigma(f)=\prod_{i}\left(T-\alpha_{\sigma, i}\right)$. For a matrix $A \in M_{n}(k)$, we define $\Gamma(A)=\Gamma(\operatorname{det}(T-A)) \in\left(k \otimes_{k_{0}} \mathbb{C}\right)^{\times}$.

Definition 4. Let $X$ be a proper smooth variety and $U \subset X$ be an open subscheme whose complement $D$ is a divisor with simple normal crossings.

(1) For a small extension $\left(\mathcal{E}_{X}, \nabla\right)$ of an integrable connection on $U$, we define the $\Gamma$-factor by

$$
\begin{array}{r}
\Gamma_{D_{i}}\left(\nabla: \mathcal{E}_{X}\right)=\Gamma\left(N_{k_{i} / k} \Phi_{\mathcal{E}_{X}, i}(T)\right)^{c_{i}} \in\left(k \otimes_{k_{0}} \mathbb{C}\right)^{\times} \\
\Gamma\left(\nabla: \mathcal{E}_{X}\right)=\prod_{i \in I} \Gamma_{D_{i}}\left(\nabla: \mathcal{E}_{X}\right) \in\left(k \otimes_{k_{0}} \mathbb{C}\right)^{\times} .
\end{array}
$$

Here $c_{i}$ is the Euler number $\chi_{c}\left(D_{i}^{*}\right)=\operatorname{dim}_{k_{i}} R \Gamma_{c}\left(D_{i}^{*}, D R\left(\mathcal{O}_{D_{i}^{*}}\right)\right)$ of $D_{i}^{*}=D_{i}-$ $\bigcup_{j \neq i} D_{j}$ and $\Phi_{\mathcal{E}_{X}, i}(T)$ denotes the characteristic polynomial of the residue of the logarithmic connection $\nabla$ at $D_{i}$.

(2) For a triple $\mathcal{M}=((\mathcal{E}, \nabla), V, \rho) \in M_{k_{0}, F}(U)$, we define the $\Gamma$-factor

$$
\Gamma(\nabla: \mathcal{M}) \in k^{\times} \backslash\left(k \otimes_{k_{0}} \mathbb{C}\right)^{\times}
$$

as the class of $\Gamma\left(\nabla: \mathcal{E}_{X}\right)$ for a small extension $\mathcal{E}_{X}$ of $\mathcal{E}$. We write

$$
\operatorname{per}_{c}^{\Gamma}(\mathcal{M})=\operatorname{per}_{c}(\mathcal{M}) \cdot \Gamma(\nabla: \mathcal{M}) .
$$

By the following Lemma, the definition (2) makes sense.

Lemma 1.8. (1) For a polynomial $f(T) \in k[T]^{\prime}$, the ratio $\Gamma(f(T)) / \Gamma(f(T-1))$ is in $k^{\times} \subset\left(k \otimes_{k_{0}} \mathbb{C}\right)^{\times}$.

(2) For extensions $\mathcal{E}_{X}^{\prime} \subset \mathcal{E}_{X}$ of an integrable connection $\mathcal{E}$, the ratio $\Phi_{\mathcal{E}_{X}, i}(T) /$ $\Phi_{\mathcal{E}_{X}^{\prime}, i}(T)$ is of the form $f(T) / f(T-1)$ for some $f(T) \in k_{i}[T]^{\prime}$. If $\mathcal{F}=\mathcal{E}_{X} / \mathcal{E}_{X}^{\prime}$ is an $\mathcal{O}_{D_{i}}$-module for an irreducible component $D_{i}$ of $D$, the residue $\operatorname{res}_{i} \nabla$ induces an $\mathcal{O}_{D_{i}}$-linear endomorphism of $\mathcal{F}$ and, if we put

$$
f(T)=\operatorname{det}\left(T-\operatorname{res}_{i} \nabla: \mathcal{F}\right),
$$

we have

$$
\Phi_{\mathcal{E}_{X}, i}(T) / \Phi_{\mathcal{E}_{X}^{\prime}, i}(T)=f(T) / f(T-1)
$$

in $k_{i}(T)$.

Proof. (1) Вy $\Gamma(s+1)=s \Gamma(s)$, it is $(-1)^{\operatorname{deg} f} f(0)^{-1} \in k^{\times}$.

(2) The second assertion follows from the exact sequence

$$
\left.\left.0 \rightarrow \mathcal{F} \otimes \mathcal{O}\left(-D_{i}\right)\left(=\mathcal{T} \operatorname{or}_{1}^{\mathcal{O}_{X}}\left(\mathcal{F}, \mathcal{O}_{D_{i}}\right)\right) \rightarrow \mathcal{E}_{X}^{\prime}\right|_{D_{i}} \rightarrow \mathcal{E}_{X}\right|_{D_{i}} \rightarrow \mathcal{F} \rightarrow 0
$$

The first assertion is deduced from the second one by the argument as in the proof of Lemma 1.2. 


\section{Relative Chow group}

(a) Fundamental properties of relative Chern classes. In this section, we define and study the relative Chow group $C H^{n}(X \bmod D)$ of dimension 0 and the relative canonical cycle $c_{X \bmod D} \in C H^{n}(X \bmod D)$. They are slight modifications of those in [S1], Section 1. Let $X$ be a smooth scheme over a field $k$. We call a finite family $D=\left(D_{i}\right)_{i \in I}$ of regular subschemes of $X$ a regular family in $X$. A typical example is the family of irreducible components of a divisor $D$ with simple normal crossings. By abuse of notation, we use the same letter $D$ for the divisor itself and for the family of its components. Let $\mathcal{K}_{n}(X)$ denote the sheaf of Quillen's K-group on $X_{Z a r}$. Namely, the Zariski sheafification of the presheaf $U \mapsto K_{n}(U)[\mathrm{Q}]$. For a regular family $D$ in $X$, let $\mathcal{K}_{n}(X \bmod D)$ be the complex $\left[\mathcal{K}_{n}(X) \rightarrow \bigoplus_{i} \mathcal{K}_{n}\left(D_{i}\right)\right]$. Here $\mathcal{K}_{n}(X)$ is put on degree 0 and $\mathcal{K}_{n}\left(D_{i}\right)$ denotes their direct image on $X$. It is the truncation at degree 1 of the complex $\mathcal{K}_{n, X, D}$ studied in [S1] and there is a natural map $\mathcal{K}_{n, X, D} \rightarrow \mathcal{K}_{n}(X \bmod D)$.

Definition 5. Let $n$ be the dimension of $X$. The $n$-th hypercohomology

$$
H^{n}\left(X, \mathcal{K}_{n}(X \bmod D)\right)
$$

of $\mathcal{K}_{n}(X \bmod D)$ is called the relative Chow group of dimension 0 and written as $C H^{n}(X \bmod D)$.

We recall the definition of the relative top Chern class. Let $\mathcal{E}$ be a locally free $\mathcal{O}_{X}$-module of rank $n$. We call a family of surjective morphisms $r_{i}:\left.\mathcal{E}\right|_{D_{i}} \rightarrow \mathcal{O}_{D_{i}}$ for $i \in I$ a partial trivialization of $\mathcal{E}$ on $D$. Let $V=\mathbb{V}(\mathcal{E})=\operatorname{Spec}\left(S\left(\mathcal{E}^{*}\right)\right)$ be the covariant vector bundle associated to $\mathcal{E}$. For $i \in I$, let $\Delta_{i}=r_{i}^{-1}(1)$, where $r_{i}:\left.V\right|_{D_{i}} \rightarrow \mathbb{A}_{D_{i}}^{1}$ is the induced map by $r_{i}:\left.\mathcal{E}\right|_{D_{i}} \rightarrow \mathcal{O}_{D_{i}}$ and $1 \subset \mathbb{A}^{1}$ is the 1 section. The family $\Delta=\left\{\Delta_{i}\right\}_{i \in I}$ is a regular family in $V$. Let $\{0\} \subset V$ denote the zero section. Then the horizontal arrows in the diagram below are isomorphisms:

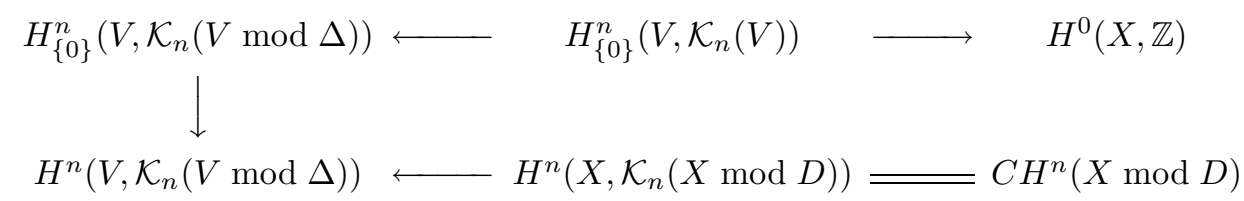

by the purity and homotopy property of K-cohomology.

Definition 6. The image of $1 \in H^{0}(X, \mathbb{Z})$ under the homomorphism $H^{0}(X, \mathbb{Z}) \rightarrow$ $C H^{n}(X \bmod D)$ is called the relative top Chern class of $(\mathcal{E}, r)$ and is denoted by $c_{n}(\mathcal{E}, r)$. Let $D$ be a divisor with simple normal crossings and $n$ the dimension of $X$. The relative canonical class $c_{X \bmod D}$ is defined as $(-1)^{n}$-times the relative top Chern class of $\left(\Omega_{X}^{1}(\log D)\right.$, res):

$$
c_{X \bmod D}=(-1)^{n} c_{n}\left(\Omega_{X}^{1}(\log D), \text { res }\right) \in C H^{n}(X \bmod D) .
$$

Here res denotes a partial trivialization of $\Omega_{X}^{1}(\log D)$ defined by the Poincaré residue $\operatorname{res}_{i}:\left.\Omega_{X}^{1}(\log D)\right|_{D_{i}} \rightarrow \mathcal{O}_{D_{i}}$.

We give some basic properties (cf. [S1], Section 1) of relative Chern classes used in the proof of the main results in Section 4. For $i \in I$, the natural map $\mathcal{K}_{n}\left(D_{i}\right)[-1] \rightarrow \mathcal{K}_{n}(X \bmod D)$ induces a map

$$
i_{i *}: H^{n-1}\left(D_{i}, \mathcal{K}_{n}\left(D_{i}\right)\right) \rightarrow H^{n}\left(X, \mathcal{K}_{n}(X \bmod D)\right) .
$$


Lemma 2.1. Let $(\mathcal{E}, \rho)$ be a locally free $\mathcal{O}_{X}$-module of rank $n$ partially trivialized on a regular family $D$. For $i \in I$, let $f_{i} \in \Gamma\left(D_{i}, \mathbb{G}_{m}\right)$ and $\sigma=\left(\sigma_{i}\right)_{i \in I}$ be another partial trivialization of $\mathcal{E}$ defined by $\sigma_{i}=f_{i}^{-1} \rho_{i}$. Then we have

$$
c_{n}(\mathcal{E}, \sigma)=c_{n}(\mathcal{E}, \rho)-\sum_{i \in I} i_{i *}\left(f_{i} \cup c_{n-1}\left(\mathcal{E}_{i}\right)\right) .
$$

Here $c_{n-1}\left(\mathcal{E}_{i}\right) \in H^{n-1}\left(D_{i}, \mathcal{K}_{n-1}\right)$ is the Chern class of the locally free $\mathcal{O}_{D_{i}}$-module $\mathcal{E}_{i}=\operatorname{Ker}\left(\rho_{i}:\left.\mathcal{E}\right|_{D_{i}} \rightarrow \mathcal{O}_{D_{i}}\right)$ and $\cup$ is the cup-product.

In Lemma 2.2, we consider a partition $I=I_{1} \amalg I_{2}$ of the index set $I$ of a regular family $D$. We put $D_{1}=\left(D_{i}\right)_{i \in I_{1}}$ and $D_{2}=\left(D_{i}\right)_{i \in I_{2}}$. There is a cup-product

$$
H^{m}\left(X, \mathcal{K}_{m}\left(X \bmod D_{1}\right)\right) \times H^{r}\left(X, \mathcal{K}_{r}\left(X \bmod D_{2}\right)\right) \rightarrow H^{n}\left(X, \mathcal{K}_{n}(X \bmod D)\right)
$$

for $n=m+r$.

Lemma 2.2. Let $0 \rightarrow \mathcal{F} \rightarrow \mathcal{E} \rightarrow \mathcal{G} \rightarrow 0$ be an exact sequence of locally free $\mathcal{O}_{X}$ modules of rank $m, n$ and $r$ respectively, and $\rho$ be a partial trivialization of $\mathcal{E}$ on a regular family $D$. Assume that on $D_{1}$, the partial trivialization $\rho$ induces a partial trivialization $\sigma=\left.\rho\right|_{\mathcal{F}}$ of $\mathcal{F}$ and that on $D_{2}$ it induces $\tau$ of $\mathcal{G}$. In other words, for $i \in I_{1}$, the restriction $\rho_{i}: \mathcal{F} \rightarrow \mathcal{O}_{D_{i}}$ is surjective and, for $i \in I_{2}$, the restriction $\rho_{i}: \mathcal{F} \rightarrow \mathcal{O}_{D_{i}}$ is 0 . Then we have

$$
c_{n}(\mathcal{E}, \rho)=c_{m}(\mathcal{F}, \sigma) \cup c_{r}(\mathcal{G}, \tau) .
$$

Lemma 2.3. Let $E$ be a regular divisor of $X$ and let $0 \rightarrow \mathcal{E} \stackrel{\varphi}{\rightarrow} \mathcal{F} \rightarrow \mathcal{G} \rightarrow 0$ be an exact sequence of locally free $\mathcal{O}_{X}$-modules $\mathcal{E}$ and $\mathcal{F}$ of the same rank $n$ and a locally free $\mathcal{O}_{E}$-module $\mathcal{G}$ of rank $m$. Let $\varphi: V=\mathbb{V}(\mathcal{E}) \rightarrow W=\mathbb{V}(\mathcal{F})$ be the associated map, $j: K \rightarrow V$ be the inclusion of the sub-vector bundle $K=\mathbb{V}\left(\left.\operatorname{Ker} \varphi\right|_{E}\right) \subset V_{E}$ of rank $m$ and $\mathcal{N}_{E / X}^{*}$ be the conormal invertible $\mathcal{O}_{E}$-module. Then we have

$$
\varphi^{*}\left(\left[0_{W}\right]\right)=\left[0_{V}\right]+j_{*}\left(\sum_{k=0}^{m-1} c_{k}(\mathcal{G}) c_{1}\left(\mathcal{N}_{E / X}^{*}\right)^{m-1-k}\right)
$$

in $H^{n}\left(V, \mathcal{K}_{n}(V \bmod \Delta)\right)$ for a regular family $\Delta \subset V$ such that $\Delta \cap\left(\left\{0_{V}\right\} \cup K\right)=\emptyset$. Here $\left[0_{V}\right]$ is the image of 1 by

$$
H^{0}\left(\left\{0_{V}\right\}, \mathbb{Z}\right) \simeq H_{\left\{0_{V}\right\}}^{n}\left(V, \mathcal{K}_{n}\right) \rightarrow H^{n}\left(V, \mathcal{K}_{n}(V \bmod \Delta)\right)
$$

and $j_{*}$ denotes the Gysin map

$$
H^{m-1}\left(K, \mathcal{K}_{m-1}\right)\left(\simeq H^{m-1}\left(E, \mathcal{K}_{m-1}\right)\right) \rightarrow H_{K}^{n}\left(V, \mathcal{K}_{n}\right) \rightarrow H^{n}\left(V, \mathcal{K}_{n}(V \bmod \Delta)\right) .
$$

Corollary 1. Let $\rho$ and $\sigma$ be partial trivializations on $\mathcal{E}$ and $\mathcal{F}$ respectively on a regular family $D$. Assume the divisor $E$ does not meet $D$ and $\left.\sigma\right|_{\mathcal{E}}=\rho$. Let $\mathcal{I}=$ $\left.\operatorname{Im} \varphi\right|_{E}$ and $i_{E *}: H^{n-1}\left(E, \mathcal{K}_{n-1}(E)\right) \rightarrow H_{E}^{n}\left(X, \mathcal{K}_{n}(X)\right) \rightarrow H^{n}\left(X, \mathcal{K}_{n}(X \bmod D)\right)$ be the Gysin map. Then we have

$$
c_{n}(\mathcal{F}, \sigma)=c_{n}(\mathcal{E}, \rho)+i_{E *}\left(c_{n-m}(\mathcal{I}) \sum_{k=0}^{m-1} c_{k}(\mathcal{G}) c_{1}\left(\mathcal{N}_{E / X}^{*}\right)^{m-1-k}\right)
$$

in $H^{n}\left(X, \mathcal{K}_{n}(X \bmod D)\right)$. 
Proof. The proof is clear from the commutative diagram

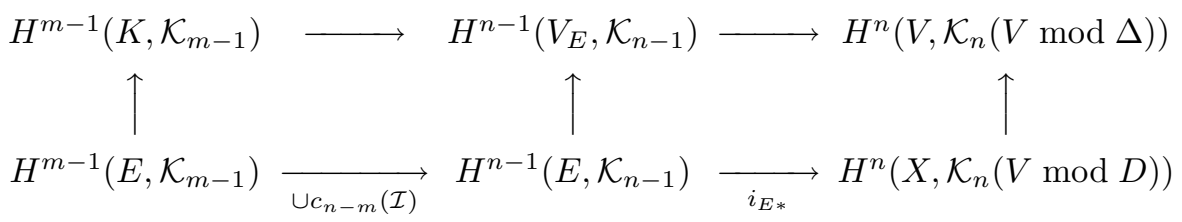

where the vertical arrows are the pull-back and the upper horizontal arrows are the Gysin maps.

In Corollary 2, we assume the regular divisor $E$ of $X$ meets transversely with $D_{i}$ for $i \in I$. Let $D_{E}$ be the regular family $\left(D_{i} \cap E\right)_{i \in I}$ and let $i_{E *}$ denote the canonical map

$$
\begin{aligned}
i_{E *}: H^{n-1}\left(E, \mathcal{K}_{n-1}\left(E \bmod D_{E}\right)\right) \simeq & H_{E}^{n}\left(X, \mathcal{K}_{n}(X \bmod D)\right) \\
& \rightarrow H^{n}\left(X, \mathcal{K}_{n}(X \bmod D)\right) .
\end{aligned}
$$

Corollary 2. Let $0 \rightarrow \mathcal{E} \stackrel{\varphi}{\rightarrow} \mathcal{F} \rightarrow \mathcal{G} \rightarrow 0$ be an exact sequence of locally free $\mathcal{O}_{X}$ modules $\mathcal{E}$ and $\mathcal{F}$ of the same rank $n$ and an invertible $\mathcal{O}_{E}$-module $\mathcal{G}$. Let $\rho$ and $\sigma$ be partial trivializations of $\mathcal{E}$ and $\mathcal{F}$ on $D$ such that $\rho=\left.\sigma\right|_{\mathcal{E}}$. Then $\sigma$ induces a partial trivialization $\left.\sigma\right|_{\mathcal{I}}$ on the locally free $\mathcal{O}_{E}$-module $\mathcal{I}=$ Image $\left.\varphi\right|_{E}$ of rank $n-1$ and we have

$$
c_{n}(\mathcal{F}, \sigma)=c_{n}(\mathcal{E}, \rho)+i_{E *} c_{n-1}\left(\mathcal{I},\left.\sigma\right|_{\mathcal{I}}\right) .
$$

In Lemma 2.4, we fix an element $0 \in I$ and assume that $E=D_{0}$ is a regular divisor of $X$ and that $E$ meets transversely with $D_{i}$ for $i \in I, \neq 0$.

Lemma 2.4. Let $0 \rightarrow \mathcal{E} \stackrel{\varphi}{\rightarrow} \mathcal{F} \rightarrow \mathcal{G} \rightarrow 0$ be an exact sequence of locally free $\mathcal{O}_{X}$ modules $\mathcal{E}$ and $\mathcal{F}$ of the same rank $n$ and locally free $\mathcal{O}_{E}$-module $\mathcal{G}$ of rank $m$. Let $\rho$ and $\sigma$ be partial trivializations of $\mathcal{E}$ and $\mathcal{F}$ on $D$ such that $\rho=\left.\sigma\right|_{\mathcal{E}}$. For $i \in I, \neq 0$, we assume that on $D_{i} \cap E$ either $\rho_{0} \oplus \rho_{i}: \mathcal{E} \rightarrow \mathcal{O}_{D_{i} \cap E}^{\oplus 2}$ is surjective or $\left.\rho_{0}\right|_{D_{i} \cap E}=\left.\rho_{i}\right|_{D_{i} \cap E}$. Then we have

$$
c_{n}(\mathcal{F}, \sigma)=c_{n}(\mathcal{E}, \rho) .
$$

Proofs of Lemmas 2.1, 2.2 and 2.3 and of Corollary 2 are done in the same way as Propositions 1, 2 and 3 and the Corollary loc.cit. respectively and we omit them. The proof of Lemma 2.4 can be simplified considerably and we give it here.

Proof of Lemma 2.4. Let $V=\mathbb{V}(\mathcal{E})$ and $W=\mathbb{V}(\mathcal{F})$ and let $\Delta_{V}$ and $\Delta_{W}$ be the regular family of closed subschemes of $V$ and $W$ defined by partial trivializations $\rho$ and $\sigma$ respectively. Since $K \cap \rho^{-1}(1)=\emptyset$, we have a commutative diagram

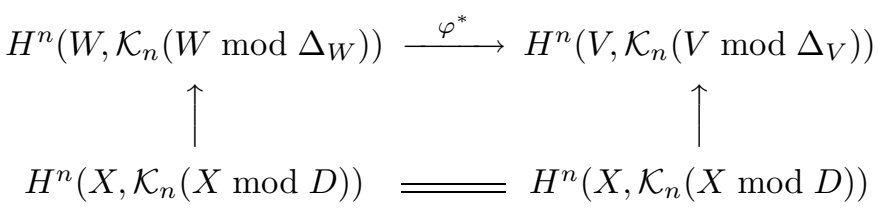

of isomorphisms. By Lemma 2.3, it is enough to show that $j_{*}: H^{m-1}\left(K, \mathcal{K}_{m-1}\right) \rightarrow$ $H^{n}\left(V, \mathcal{K}_{n}\left(V \bmod \Delta_{V}\right)\right)$ is the 0-map. We consider a complex

$$
\mathcal{K}_{n}(V \bmod \Delta)^{\prime}=\left[\mathcal{K}_{n}(V) \rightarrow \bigoplus_{i \in I} \mathcal{K}_{n}\left(\Delta_{i}\right) \rightarrow \bigoplus_{i \in I, \neq 0} \mathcal{K}_{n}\left(\Delta_{i} \cap \Delta_{0}\right)\right] .
$$


Since $j_{*}$ factors $H^{n}\left(V, \mathcal{K}_{n}(V \bmod \Delta)^{\prime}\right)$, it is enough to show $j_{*}: H^{m-1}\left(K, \mathcal{K}_{m-1}\right) \rightarrow$ $H^{n}\left(V, \mathcal{K}_{n}\left(V \bmod \Delta_{V}\right)^{\prime}\right)$ is the 0-map. It is proved by the same argument as in the last 6 lines of the first paragraph of the proof of Proposition 4 loc.cit.

(b) Adelic presentation. In the rest of this section, we give an adelic presentation of the form

$$
C H^{n}(X \bmod D) \simeq \operatorname{Coker}\left(\partial: \bigoplus_{y \in X_{1}} B_{y} \rightarrow \bigoplus_{x \in X_{0}} A_{x}\right) .
$$

Here $X_{i}$ denotes the set of the points of $X$ of dimension $i$.

To define the right hand side, we first introduce some terminology on the tame symbol. For a vector space $L$ of dimension 1 over a field $K$, we put $A(L)=$ $\left(\bigoplus_{m \in \mathbb{Z}} L^{\otimes m}\right)^{\times}$. It is an extension of $\mathbb{Z}$ by $K^{\times}$since the algebra $\bigoplus_{m \in \mathbb{Z}} L^{\otimes m}$ is isomorphic to the Laurent polynomial ring $K\left[T, T^{-1}\right]$. Its underlying set is the disjoint union $\amalg_{m \in \mathbb{Z}}\left\{K\right.$-basis of $\left.L^{\otimes m}\right\}$. Let $R$ be a noetherian local integral ring of dimension 1 such that the normalization $\tilde{R}$ is finite over $R$. Let $K$ be the fraction field and $\kappa$ be the residue field of $R$. We review the definition of the order ord : $K^{\times} \rightarrow \mathbb{Z}$ and the tame symbol $\partial: K_{2}(K) \rightarrow \kappa^{\times}$. For a maximal ideal $\tilde{x}$ of $\tilde{R}$, let $\operatorname{ord}_{\tilde{x}}: K^{\times} \rightarrow \mathbb{Z}$ and $\partial_{\tilde{x}}: K_{2}(K) \rightarrow \kappa(\tilde{x})^{\times}$be the usual order and the tame symbol

$$
\partial_{\tilde{x}}(\{f, g\})=(-1)^{\operatorname{ord} \tilde{x} f \cdot \operatorname{ord} \tilde{x} g} f^{\operatorname{ord} \tilde{x} g} g^{-\operatorname{ord} \tilde{x} f}(\tilde{x})
$$

for the discrete valuation ring $\tilde{R}_{\tilde{x}}$. Then ord $=\sum_{\tilde{x} \mapsto x}[\kappa(\tilde{x}): \kappa] \operatorname{ord}_{\tilde{x}}$ and $\partial=$ $\prod_{\tilde{x} \mapsto x} N_{\tilde{x} / x} \circ \partial_{\tilde{x}}$. For an invertible $R$-module $L$, we define a pairing

$$
(,): A\left(L_{K}\right) \times K^{\times} \rightarrow A\left(L_{\kappa}\right)
$$

generalizing the tame symbol as follows. We put $A(L)=\amalg_{m \in \mathbb{Z}}\left\{R\right.$-basis of $\left.L^{\otimes m}\right\}$ with the structure of abelian group defined by the tensor product. The abelian group $A\left(L_{K}\right)$ is the amalgamated sum $A(L) \oplus_{R^{\times}} K^{\times}$. We define the pairing by requiring that, on $A(L) \times K^{\times}$, it is the composition of (the reduction) $\times$ord : $A(L) \times K^{\times} \rightarrow A\left(L_{\kappa}\right) \times \mathbb{Z}$ and the obvious pairing and that, on $K^{\times} \times K^{\times}$, it is the tame symbol $\partial: K^{\times} \times K^{\times} \rightarrow \kappa^{\times}$. Since they coincide on $R^{\times} \times K^{\times}$, the pairing is well-defined.

The pairing $():, A\left(L_{K}\right) \times K^{\times} \rightarrow A\left(L_{\kappa}\right)$ is computed on the normalization $\tilde{R}$ as follows. Let $\tilde{x}$ be a maximal ideal of $\tilde{R}$. The tame symbol $(,)_{\tilde{x}}: A\left(L_{K}\right) \times$ $K^{\times} \rightarrow A\left(L_{\kappa(\tilde{x})}\right)$ is defined in the same way as above. We also have the norm map $A\left(L_{\kappa(\tilde{x})}\right) \rightarrow A\left(L_{\kappa}\right)$. A basis $\ell$ of $L_{\kappa(\tilde{x})}^{\otimes e}$ is of the form $a \cdot \ell_{0}^{\otimes e}$ for $a \in \kappa(\tilde{x})^{\times}$ and a basis $\ell_{0}$ of $L_{\kappa}$. The basis $N(\ell)=N_{\kappa(\tilde{x}) / \kappa}(a) \cdot \ell_{0}^{\otimes e \cdot[\kappa(\tilde{x}): \kappa]}$ of $L_{\kappa}^{\otimes e \cdot[\kappa(\tilde{x}): \kappa]}$ is independent of the choice of $\ell_{0}$ and well-defined. The norm map $\ell \mapsto N(\ell)$ defines a homomorphism $N_{\kappa(\tilde{x}) / x}: A\left(L_{\kappa(\tilde{x})}\right) \rightarrow A\left(L_{\kappa}\right)$ and the diagram

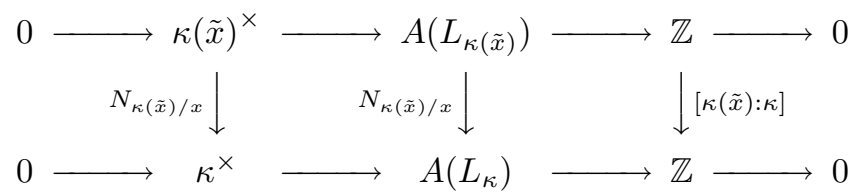

is commutative. We have

$$
(,)_{\kappa}=\prod_{\tilde{x}} N_{\kappa(\tilde{x}) / \kappa} \circ(,)_{\tilde{x}}
$$


Let $X$ and $D$ be as before and we define $A_{x}$ for $x \in X$. For $x \in X$, we put $I_{x}=\left\{i ; x \in D_{i}\right\}$. For $i \in I_{x}$, let $N_{i}(x)$ be the one-dimensional $\kappa(x)$-vector space $\mathcal{O}_{X}\left(-D_{i}\right) \otimes \kappa(x)$. We put $A_{x, i}=A\left(N_{i}(x)\right)$ and write the canonical map $\operatorname{ord}_{i}: A_{x, i} \rightarrow \mathbb{Z}$. The abelian group $A_{x}$ is the fiber product $\left(\prod_{i \in I_{x}}\right)_{\mathbb{Z}} A_{x, i}$ with respect to $\operatorname{ord}_{i}$. It is an extension of $\mathbb{Z}$ by $\bigoplus_{i \in I_{x}} \kappa(x)^{\times}$. We define $B_{y}$ for $y \in X$. For $i \in I_{y}$, let $B_{y, i}$ be the amalgamated $\operatorname{sum}\left(A_{y, i} \otimes \kappa(y)^{\times}\right) \oplus_{\kappa(y) \times \otimes \kappa(y) \times} K_{2}(y)$. It is an extension of $\kappa(y)^{\times}$by $K_{2}(y)$. The group $B_{y}$ is defined as the fiber product $\left(\prod_{i \in I_{y}}\right)_{\kappa(y) \times} B_{y, i}$ with respect to ord $\otimes i d: B_{y, i} \rightarrow \kappa(y)^{\times}$.

The homomorphism $\partial$ is the direct sum of the $(x, y)$-components $\partial_{x, y}: B_{y} \rightarrow A_{x}$ for $x \in X_{0}$ and $y \in X_{1}$. This fits in the commutative diagram

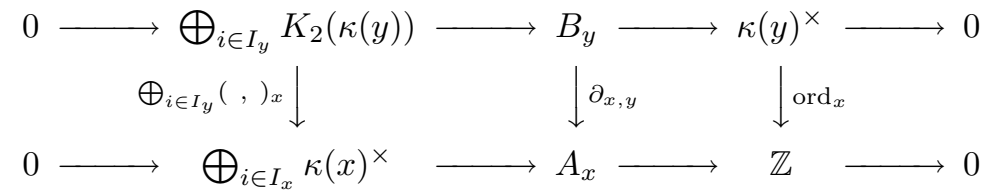

and is 0 unless $x$ is not in the closure $Y$ of $\{y\}$. Here $\operatorname{ord}_{x}: \kappa(y)^{\times} \rightarrow \mathbb{Z}$ is the usual order and $(,)_{x}: K_{2}(\kappa(y)) \rightarrow \kappa(x)^{\times}$is the tame symbol. The local component $\partial_{x, y}: B_{y} \rightarrow A_{x}$ is determined by its $i$-th components $\partial_{x, y, i}: B_{y, i} \rightarrow A_{x, i}$ for $i \in I_{y}$ and $\partial_{x, y, i}: \kappa(y)^{\times} \rightarrow A_{x, i}$ for $i \in I_{x}-I_{y}$. We use the tame symbol (, ) defined above for the local ring $\mathcal{O}_{Y, x}$ and the invertible module $\mathcal{O}\left(-D_{i}\right)$. For $i \in I_{y}$, the $i$-th component $\partial_{x, y, i}$ is induced by the pairing $():, A\left(N_{i}(y)\right) \times \kappa(y)^{\times} \rightarrow A\left(N_{i}(x)\right)=$ $A_{x, i}$. For $i \in I_{x}-I_{y}$, it is defined as $(-1)^{\operatorname{ord}_{x}} \times(1):, \kappa(y)^{\times} \rightarrow A\left(N_{i}(x)\right)=A_{x, i}$. Here $(1$,$) is the pairing with 1$ regarded as a $\kappa(y)$-basis of $\mathcal{O}\left(-D_{i}\right) \otimes \kappa(y)$ which is an element of $A\left(\mathcal{O}\left(-D_{i}\right) \otimes \kappa(y)\right)$. It is clear that we have a commutative diagram

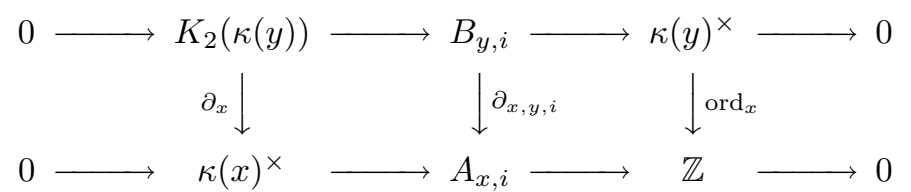

for $i \in I_{y}$ and that the composition $\kappa(y) \stackrel{\partial_{x, y, i}}{\rightarrow} A_{x, i} \stackrel{\text { ord }_{i}}{\longrightarrow} \mathbb{Z}$ is $\operatorname{ord}_{x}$ for $i \in I_{x}-I_{y}$. Hence $\partial_{x, y}: B_{y} \rightarrow A_{x}$ is defined and satisfies the commutative diagram above.

We give an explicit computation of $\partial_{x, y}$. For an inverse image $\tilde{x}$ of $x$ in the normalization $\tilde{Y}$ of the closure $Y$ of $\{y\}$, let $A_{\tilde{x}, i}=A\left(N_{i}(x) \otimes_{\kappa(x)} \kappa(\tilde{x})\right)$ and define the fiber product $A_{\tilde{x}}=\prod_{i \in I_{x} \mathbb{Z}} A_{\tilde{x}, i}$ and the boundary map $\partial_{\tilde{x}, y}: B_{y} \rightarrow A_{\tilde{x}}$ in the same way as above. The norm maps defined above induce $N_{\kappa(\tilde{x}) / \kappa(x)}: A_{\tilde{x}} \rightarrow A_{x}$ and we have

$$
\partial_{x, y}=\prod_{\tilde{x}} N_{\kappa(\tilde{x}) / \kappa(x)} \partial_{\tilde{x}, y} .
$$

We compute $\partial_{\tilde{x}, y}$ explicitly by taking a basis $\pi_{i}$ of $\left.\mathcal{O}\left(-D_{i}\right)\right|_{Y}$ at $x$ for $i \in I_{x}$. The basis defines splittings $A_{\tilde{x}}=\mathbb{Z} \oplus \bigoplus_{i \in I_{x}} \kappa(\tilde{x})^{\times}$and $B_{y}=\kappa(y)^{\times} \oplus \bigoplus_{i \in I_{y}} K_{2}(y)$ by $1 \mapsto \pi_{i}$. Since the other components of $\partial_{\tilde{x}, y}$ are evident, we compute the component $\kappa(y)^{\times} \rightarrow \kappa(\tilde{x})^{\times}$. If $i \in I_{y}$, it is 0 and if $i \in I_{x}-I_{y}$, it is given by

$$
f \mapsto f /\left.\pi_{i}^{\operatorname{ord} \tilde{x} f}\right|_{\tilde{x}}=\left(-\pi_{i}, f\right)_{\tilde{x}}^{-1} .
$$

In fact in the latter case, the rational section 1 of $\left.\mathcal{O}\left(-D_{i}\right)\right|_{Y}$ is $\pi_{i}^{-1}$-times the basis $\pi_{i}$. 
Proposition 1. Let $X$ be a smooth scheme of dimension $n$ over a field $k$ and $D$ be a divisor with simple normal crossings. Under the notation above, there exists a canonical isomorphism

$$
C H^{n}(X \bmod D) \simeq \operatorname{Coker}\left(\partial: \bigoplus_{y \in X_{1}} B_{y} \rightarrow \bigoplus_{x \in X_{0}} A_{x}\right) .
$$

The proof will be given in the Appendix.

Corollary. Let $U^{\prime}$ be a dense open subscheme of $U$. The relative Chow group $C H^{n}(X \bmod D)$ is generated by the classes $[x]$ for $x \in U_{0}^{\prime}$.

Proof of the Corollary. First we assume $U^{\prime}=U$. Let $U_{m}=\left\{x \in X\right.$; Card $\left.I_{x} \leq m\right\}$. We have $X=U_{n}$ and $U=U_{0}$. By induction on $m$, it is sufficient to show that the image of $\bigoplus_{x \in U_{m}} A_{x}$ in $C H_{0}(X, D)$ is in that of $\bigoplus_{x \in U_{m-1}} A_{x}$ for $m \geq 1$. Let $x \in X_{0}$ such that Card $I_{x}=m \geq 1$. We take a basis $\pi_{i}$ of $N_{i}(x)=\mathcal{O}\left(-D_{i}\right)(x)$ for each $i \in$ $I_{x}$. It defines a splitting $A_{x}=\mathbb{Z} \oplus \bigoplus_{i \in I_{x}} \kappa(x)^{\times}$as above. Let $f \in A_{x}$ be an arbitrary element and write it as a sum $f=\sum_{i} f_{i}$, where $f_{i} \in \mathbb{Z} \oplus\left(i\right.$-th component of $\left.\kappa(x)^{\times}\right)$. For each $i \in I_{x}$, we put $I_{i}=I_{x}-\{i\}$ and take a closed integral curve $Y_{i} \subset D_{I_{i}}, \ni x$ regular at $x$ and meeting $D_{i}$ transversally at $x$. Let $y_{i}$ be the generic point of $Y_{i}$ and $S_{i} \subset Y_{i}$ be the closed subset $S_{i}=\bigcup_{j \in I-I_{i}}\left(Y_{i} \cap D_{j}\right)$ of $Y_{i}$. Let $f$ be an arbitrary element of $A_{x}$. We take a rational function $g_{i} \in \kappa\left(y_{i}\right)^{\times}$for $i \in I_{x}$ and a rational section $\pi_{i, j}$ of $\left.\mathcal{O}\left(-D_{j}\right)\right|_{Y_{i}}$ for $i \neq j, \in I_{x}$ satisfying the following conditions.

The pair $\left(\operatorname{ord}_{x} g_{i},\left(-\pi_{i}, g_{i}\right)_{x}^{-1}\right) \in \mathbb{Z} \times \kappa(x)^{\times}$is equal to $f_{i}$ and the pull-back of $g_{i}$ to the normalization of $Y_{i}$ is invertible and congruent to 1 at the inverse image of $S_{i}-\{x\}$. The section $\pi_{i, j}$ is a basis of $\left.\mathcal{O}\left(-D_{j}\right)\right|_{Y_{i}}$ at $S_{i}$ and the fiber $\pi_{i, j}(x)$ is $\pi_{j}$ for $j \neq i$.

Let $h_{i} \in B_{y_{i}}$ be the element defined by $\left(\pi_{i, j}\right)_{j} \otimes g_{i}$. Then by the explicit computation given above, we see $f_{i}=\partial_{x, y_{i}} h_{i}$. Since $\partial\left(\bigoplus_{i \in I_{x}} h_{i}\right)$ is supported on $\{x\} \cup \bigcup_{i}\left(Y_{i}-S_{i}\right)$ and $Y_{i}-S_{i}=Y_{i} \cap U_{m-1}$, the Corollary for $U^{\prime}=U$ is proved.

To prove the Corollary for general $U^{\prime}$, it is sufficient to show that the image of $\bigoplus_{x \in U} \mathbb{Z}$ in $C H_{0}(X, D)$ is in that of $\bigoplus_{x \in U^{\prime}} \mathbb{Z}$. For a closed point $x \in U-U^{\prime}$, take a closed integral curve $Y \ni x$ regular at $x$ and meeting $U$, and let $y$ be the generic point of $Y$. Let $f$ be an arbitrary integer. Take a rational function $g \in$ $\kappa(y)^{\times}=B_{y}$ such that $\operatorname{ord}_{x} g=f$ and that the pull-back of $g$ to the normalization of $Y$ is invertible and congruent to 1 at the inverse image of $Y-U^{\prime}$. Then we have $\partial_{x, y}(g)=f$ and $\partial(g)$ is supported on $\{x\} \cup\left(Y \cap U^{\prime}\right)$. Thus the Corollary is proved.

\section{TAME SYMBOL}

(a) The definition of pairing. Let $k_{0}, F \subset \mathbb{C}$ be subfields and $k$ be a finite extension of $k_{0}$. Let $X$ be a proper smooth scheme over $k$ and $U$ be the complement of a divisor $D$ of $X$ with simple normal crossings as in Section 1. Recall that $M \operatorname{Pic}_{k_{0}, F}(U)$ denotes the class group of $P_{k_{0}, F}(U)$. The purpose of this section is to define a canonical pairing

$$
\begin{aligned}
(, \quad): M P i c_{k_{0}, F}(U) \otimes C H^{n}(X \bmod D) \rightarrow & M P i c_{k_{0}, F}(k) \\
& \simeq k^{\times} \backslash\left(k \otimes_{k_{0}} \mathbb{C}\right)^{\times} /\left(F^{\times}\right)^{\text {Hom }_{k_{0}}(k, \mathbb{C}) .}
\end{aligned}
$$

First, for a closed point $x \in X_{0}$, we define the local pairing:

$$
(,)_{x}: M P i c_{k_{0}, F}(U) \times A_{x} \rightarrow M P i c_{k_{0}, F}(x) .
$$


Let $A_{I_{x}}$ be the inverse image of the diagonal $\mathbb{Z} \rightarrow \mathbb{Z}^{I_{x}}$ by ord, where ord is the product of order homomorphisms:

$$
\prod_{i \in I_{x}} \Gamma\left(X_{x}-D_{i}, \mathbb{G}_{m}\right) \stackrel{\prod_{i} \text { ord }_{i}}{\rightarrow} \prod_{i \in I_{x}} \mathbb{Z}
$$

Here $X_{x}$ is the spectrum $\operatorname{Spec} \mathcal{O}_{X, x}$ of the local ring $\mathcal{O}_{X, x}$. Considering the pushout diagram

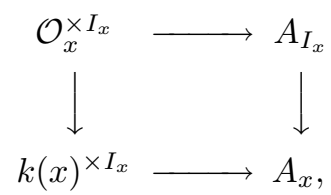

to define the pairing ( , ), it is enough to define $M P i c_{k_{0}, F}(U) \times A_{I_{x}} \rightarrow M P i c_{k_{0}, F}(x)$ whose restriction to $\mathcal{O}_{X, x}^{\times I_{x}}$ depends only on the image to $k(x)^{\times I_{x}}$.

For an object $\mathcal{M}=((\mathcal{E}, \nabla), V, \rho)$ of $P_{k_{0}, F}(U)$, we choose an extension $\left(\mathcal{E}_{X}, \nabla_{X}\right)$ of $(\mathcal{E}, \nabla)$ with regular singularity. The $\mathcal{O}_{X}$-module $\mathcal{E}_{X}$ is invertible. For a local basis $e$ of $\mathcal{E}_{X}$, we let $\nabla \log e$ denote the local section of $\Omega_{X}^{1}(\log D)$ satisfying $\nabla e=$ $e \otimes \nabla \log e$. Let $\nabla_{i}$ be

$$
\operatorname{res}_{i}\left(\nabla: \mathcal{E}_{X}\right)=\operatorname{res}_{i} \nabla \log e \in \operatorname{End}_{\mathcal{O}_{D_{i}}}\left(\left.\mathcal{E}_{X}\right|_{D_{i}}\right)=\Gamma\left(D_{i}, \mathcal{O}_{D_{i}}\right) .
$$

For an element $f=\left(f_{i}\right)$ in $A_{I_{x}}$, we define a triple

$$
\left(\left(\mathcal{E}_{X}^{\otimes \operatorname{ord}_{x} f},(\nabla, f)^{\sim}\right),(V, f)^{\sim},(\rho, f)^{\sim}\right)
$$

in an etale neighborhood $\tilde{X} \ni x$ of $x$ and we define $(\mathcal{M}, f)_{x} \in M P i c_{k_{0}, F}(X)$ to be the isomorphism class of its restriction. We define an integrable connection $(\nabla, f)^{\sim}$ on $\mathcal{E}_{X}^{\otimes \operatorname{ord}_{x} f}$ by

$$
(\nabla, f)^{\sim} \log \left(e^{\otimes \operatorname{ord}_{x} f}\right)=\operatorname{ord}_{x} f \nabla \log e-\sum_{i \in I_{x}} \nabla_{i} d \log f_{i} .
$$

Since the residues of the connection are 0 at $x$, it is extended to a connection in a neighborhood of $x$. We define a local system $(V, f)^{\sim}$ of $F$-vector spaces on $U^{a n}$ as a subsheaf of $\mathcal{E}^{a n \otimes \text { ord } f}$ by

$$
(V, f)^{\sim}=(-1)^{-\operatorname{ord} f \cdot \nabla} f^{\nabla} \rho(V)^{\otimes \text { ord } f} \subset \mathcal{E}^{a n \otimes \operatorname{ord} f}
$$

where

$$
(-1)^{-\operatorname{ord} f \cdot \nabla} f^{\nabla}=\prod_{i \in I_{x}}(-1)^{-\operatorname{ord} f \cdot \nabla_{i}} f_{i}^{\nabla_{i}}=\exp \left(\sum_{i \in I_{x}} \nabla_{i} \cdot\left(-\operatorname{ord} f \cdot \pi \sqrt{-1}+\log f_{i}\right)\right) .
$$

It may seem to depend on the choice of a branch of the logarithm. However, since $\exp \left(2 \pi \sqrt{-1} \nabla_{i}\right)$ is the inverse of the action of the local monodromy along $D_{i}$, it is in $F$ by [D1], II, Proposition 3.11. Therefore $(V, f)^{\sim}$ is well-defined as a local system of $F$-vector spaces of rank 1. Since $d \log (-1)^{-\operatorname{ord} f \cdot \nabla} f^{\nabla}=\sum_{i \in I_{x}} \nabla_{i} d \log f_{i}$, the local system $\mathbb{C} \otimes_{F}(V, f)^{\sim}$ is the sheaf of flat sections of $\left(\mathcal{E}^{a n \otimes \text { ord } f},(\nabla, f)^{\sim}\right)$. Hence $(V, f)^{\sim}$ is extended to a local system on a neighborhood of $x^{a n}$ in $X^{a n}$. We let $(\rho, f)^{\sim}$ denote the natural inclusion $(V, f)^{\sim} \rightarrow \mathcal{E}_{X}^{a n \otimes \operatorname{ord} f}$. By restricting to $x^{a n}$, we obtain a triple $(\mathcal{M}, f)_{x}=\left(\mathcal{E}_{X}^{\otimes \operatorname{ord}_{x} f} \otimes k(x),\left.(V, f)^{\sim}\right|_{x^{a n}},\left.(\rho, f)^{\sim}\right|_{x^{a n}}\right)$ and we use the same symbol $(\mathcal{M}, f)_{x} \in M P i c_{k_{0}, F}(X)$ to denote its isomorphism class. It is easily checked that the isomorphism class is independent of the choice of an extension $\mathcal{E}_{X}$ and also depends only on the image of $f$ in $A_{x}$. 
The tame symbol

$$
(\mathcal{M}, f)_{x} \in M P i c_{k_{0}, F}(k(x)) \simeq k(x)^{\times} \backslash\left(k(x) \otimes_{k_{0}} \mathbb{C}\right)^{\times} /\left(F^{\times}\right)^{\operatorname{Hom}_{k_{0}}(k(x), \mathbb{C})}
$$

can be computed explicitly as follows. Let $e$ be a local basis of $\mathcal{E}_{X}$ at $x$ and $v$ be a (multivalued) local basis of $V$ on $W-D^{a n}$ for a sufficiently small neighborhood $W$ of $x$ in $X^{a n}$. Set $\varphi$ as the analytic (multivalued) function $\rho(v) / e$ on $W-D^{a n}$. We define $(\varphi, f)^{\sim}$ to be

$$
(\varphi, f)^{\sim}=(-1)^{-\operatorname{ord} f \cdot \operatorname{res} \nabla \log e} f^{\operatorname{res} \nabla \log e} \times \varphi^{\text {ord } f},
$$

where

$$
\begin{aligned}
& (-1)^{-\operatorname{ord} f \cdot \operatorname{res} \nabla \log e} f^{\mathrm{res} \nabla \log e} \\
= & \exp \left(\sum_{i \in J} \operatorname{res}_{i}(\nabla \log e) \cdot\left(-\operatorname{ord} f \cdot \pi \sqrt{-1}+\log f_{i}\right)\right)
\end{aligned}
$$

for some choice of branch of the logarithm. Note that $(\varphi, f)^{\sim}$ is well-defined up to a multiplicative constant in $F^{\times}$by the same reason as above. Since $\rho(v)$ is a horizontal section, $\varphi$ satisfies the differential equation $\nabla \log e=-d \log \varphi$. Hence the logarithmic differential

$$
d \log (\varphi, f)^{\sim}=\sum_{i \in J} \operatorname{res}_{i}(\nabla \log e) \cdot d \log f_{i}+\operatorname{ord} f \cdot d \log \varphi
$$

does not have a pole on $U$. Therefore $\log (\varphi, f)^{\sim}$ is a holomorphic function and $(\varphi, f)^{\sim}$ is invertible and holomorphic. The inverse class of the value $(\varphi, f)_{x}$ of $(\varphi, f)^{\sim}$ at $x^{a n}$ gives the tame symbol

$$
(\mathcal{M}, f) \in k(x)^{\times} \backslash\left(k(x) \otimes_{k_{0}} \mathbb{C}\right)^{\times} /\left(F^{\times}\right)^{\operatorname{Hom}_{k_{0}}(k(x), \mathbb{C})} .
$$

Now we define the global pairing. For a finite extension $L / k$, the norm map $\left(L \otimes_{k_{0}} \mathbb{C}\right)^{\times} \rightarrow\left(k \otimes_{k_{0}} \mathbb{C}\right)^{\times}$induces a norm map $N_{L / k}: M P i c_{k_{0}, F}(L) \rightarrow M P i c_{k_{0}, F}(k)$. For an element $\left(p_{\tau}\right)_{\tau: L \rightarrow \mathbb{C}}$ in $M P i c_{k_{0}, F}(L)$, the $\sigma: k \rightarrow \mathbb{C}$ component $N_{L / k}\left(\left(p_{\tau}\right)\right)_{\sigma}$ is given by $\prod_{\left.\tau\right|_{k}=\sigma} p_{\tau}$. By composing with the norm $N_{x / k}: M P i c_{k_{0}, F}(x) \rightarrow$ $M P i c_{k_{0}, F}(k)$, we obtain a pairing

$$
(,)_{X}=\prod_{x \in X_{0}} N_{x / k} \circ(,)_{x}: M P i c_{k_{0}, F}(U) \times \bigoplus_{x \in X_{0}} A_{x} \rightarrow M P i c_{k_{0}, F}(k) .
$$

Proposition 2. Assume further that $X$ is projective and $n=\operatorname{dim} X$. Then the pairing $(,)_{X}: M P i c_{k_{0}, F}(U) \times \bigoplus_{x \in X_{0}} A_{x} \rightarrow M P i c_{k_{0}, F}(k)$ annihilates the image of $\partial: \bigoplus_{y \in X_{1}} B_{y} \rightarrow \bigoplus_{x \in X_{0}} A_{x}$.

Definition 7. By Proposition 2 and the adelic presentation, Proposition 1,

$$
C H^{n}(X \bmod D)=\operatorname{Coker}\left(\partial: \bigoplus_{y \in X_{1}} B_{y} \rightarrow \bigoplus_{x \in X_{0}} A_{x}\right),
$$

the pairing $(,)_{X}$ induces a pairing

$$
(,)_{X}: M P i c_{k_{0}, F, X}(U) \times C H^{n}(X \bmod D) \rightarrow M P i c_{k_{0}, F}(k) .
$$

This pairing is called the tame symbol.

Proof. First we prove Proposition 2 for curves and then reduce the general case to it. 
(b) One-dimensional case. Assume $X$ is a curve. We may assume $X$ is integral and $y$ is the generic point of $X$. The group $B_{y}$ is then the function field $\kappa(y)^{\times}$. Hence it is enough to show that for an object $\mathcal{M}=((\mathcal{E}, \nabla), V, \rho)$ of $P_{k_{0}, F}(U)$ and for $f \in k(X)^{\times}$, we get

$$
(\mathcal{M}, \partial f)_{X}=1
$$

First we consider the case where a function $f \in k(X)^{\times}$is constant. By considering the norm from the constant field to $k$, we may assume that the constant field of $X$ is $k$. We take a branch of the $\operatorname{logarithm} \log f \in \mathbb{C}(1)^{\text {Hom }_{k_{0}}(k, \mathbb{C})}$ of $f \in k^{\times}$. Let $\mathcal{M}=((\mathcal{E}, \nabla), V, \rho)$ be an object of $P_{k_{0}, F}(U)$. Let $e$ be a non-zero rational section of $\mathcal{E}$. For $x \in D$, let $\nabla_{x}=\operatorname{res}_{x} \nabla \log e \in \kappa(x)$ and $\left[f^{\nabla_{x}}\right]$ be the class of $f^{\nabla_{x}}$ in $M P i c_{k_{0}, F}(x)$ where $f^{\nabla_{x}}=\exp \left(\nabla_{x} \cdot \log f\right) \in\left(\kappa(x) \otimes_{k_{0}} \mathbb{C}\right)^{\times}$. Note that $\partial_{x} f \neq 0$ if and only if $x \in D$ and then $\partial_{x} f=f \in \kappa(x)^{\times} \subset A_{x}$. Then by the definition of $\partial_{x}$, $\left[f^{\nabla_{x}}\right]=\left(\mathcal{M}, \partial_{x} f\right)$. Therefore it is enough to prove that $\prod_{x \in D} N_{x / k}\left(\left[f^{\nabla_{x}}\right]\right)=1$ in $M$ Pic $_{k_{0}, F}(k)$. We have $\sum_{x \in D} \operatorname{Tr}_{x / k} \nabla_{x}=-\sum_{x \notin D}[\kappa(x): k] \operatorname{ord}_{x}$ e by the residue formula $\sum_{x \in X} \operatorname{Tr}_{x / k} \operatorname{res}_{x} \nabla \log e=0$ and by $\operatorname{ord}_{x} e=\operatorname{res}_{x} \nabla \log e$ for $x \notin D$. Since $\operatorname{ord}_{x} e \in \mathbb{Z}$, we get the required identity.

We assume that a function $f \in k(X)^{\times}$is not constant. It defines a finite flat morphism $f: X \rightarrow Y=\mathbb{P}_{k}^{1}$ denoted by the same character. By shrinking $U$ if necessary, we may assume that $U=f^{-1}(W)$ for $W=f(U)$ and that $f: U \rightarrow W$ is finite and etale. We put $E=\mathbb{P}_{k}^{1}-W$ and let $t$ be the coordinate of $\mathbb{P}_{k}^{1}$. We reduce to the case $X=\mathbb{P}^{1}$ and $f=t$ using norm argument.

First we define the norm functor for a finite flat morphism $f: X \rightarrow Y$ of proper smooth curves over $k$. Let $W \subset Y$ be an open subscheme such that $U=f^{-1}(W) \rightarrow$ $W$ is finite etale and let the reduced divisors $E=Y-W$ and $D=X-U$ be the complements. We define the norm functor

$$
N_{X / Y}: P_{k_{0}, F}(U) \rightarrow P_{k_{0}, F}(W) .
$$

Let $\mathcal{M}=((\mathcal{E}, \nabla), V, \rho)$ be an object of $P_{k_{0}, F}(U)$. The invertible $\mathcal{O}_{W}$-module $N(\mathcal{E})$ is given in [D7], 7.1. For a basis $e$ of $\mathcal{E}$ on the inverse image $f^{-1}(S)$ of an open subset $S \subset W$, the invertible $\mathcal{O}_{Y}$-module $N(\mathcal{E})$ is generated by the symbol $N(e)$ on $S$. For another local basis $e^{\prime}=g e$, we require $N\left(e^{\prime}\right)=N_{X / Y}(g) N(e)$. We define a connection $\nabla$ on $N(\mathcal{E})$ by $\nabla \log N e=\operatorname{Tr}_{X / Y} \nabla \log e$. It is clear from this that $(N(\mathcal{E}), \nabla)$ is regular along $E$. The local system $N(V)$ is defined similarly. For a basis $v$ of $V$ on the inverse image $f^{-1}(S)$ of an open subset $S \subset W^{a n}$, the local system $N(V)$ is locally generated by the symbol $N(v)$ on $S$. For another local basis $v^{\prime}=g v$, we impose $N\left(v^{\prime}\right)=N_{X / Y}(g) N(v)$. The comparison map $N(\rho)$ is defined by $N(\rho) N(v)=N_{X / Y}(\varphi) N(e)$ for an analytic function $\varphi$ such that $\rho(v)=\varphi e$. It is easily checked that the norm is an additive functor.

For the reduction to $X=\mathbb{P}^{1}, f=t$, it is enough to show $(\mathcal{M}, \partial f)_{X}=\left(N_{f} \mathcal{M}, \partial t\right)_{Y}$, by taking $f: X \rightarrow Y=\mathbb{P}^{1}$. By the definition of the tame symbol, it is reduced to a local equality $\prod_{x \mapsto y} N_{x / y}(\mathcal{M}, f)_{x}=\left(N_{f} \mathcal{M}, t\right)_{y} \in M P i c_{k_{0}, F}(y)$. Thus we are led to study compatibility of the tame symbol and the norm. Since $A_{x}=k(X)^{\times} / 1+m_{x}$ if $x \in D$ and $=\mathbb{Z}$ otherwise, there is a natural map $f^{*}: A_{y} \rightarrow A_{x}$ for $y=f(x)$. It induces the multiplication by the ramification index $m$ on the quotient $\mathbb{Z}$. The following Lemma will reduce the proof for curves to the case where $X=\mathbb{P}^{1}, f=t$.

Lemma 3.1. Let $f: X \rightarrow Y$ be a finite flat morphism of proper smooth curves over $k$, inducing a finite etale morphism $f: U \rightarrow W$ on dense open subschemes 
and let $y$ be a closed point of $Y$. For $\mathcal{M}=((\mathcal{E}, \nabla), V, \rho) \in P_{k_{0}, F}(U)$, we have

$$
\left(N_{X / Y} \mathcal{M}, t\right)_{y}=\prod_{f(x)=y} N_{x / y}\left(\mathcal{M}, f^{*} t\right)_{x}
$$

Proof. We choose a regular singular extension $\left(\mathcal{E}_{X}, \nabla_{X}\right)$ of $(\mathcal{E}, \nabla)$ and take and fix a local basis $e$ of $\mathcal{E}_{X}$ at $f^{-1}(y)$. Let $v$ be a multivalued local section of $V$ on $f^{-1}\left(W-y^{a n}\right)$ where $W$ is a sufficiently small neighborhood of $y^{a n}$, and let $\varphi=\rho(v) / e$ be a multivalued analytic function on $f^{-1}\left(W-y^{a n}\right)$ as in the definition of the tame symbol. We prove

$$
\left(N_{X / Y} \varphi, t\right)_{y}=\prod_{f(x)=y} N_{x / y}\left(\varphi, f^{*} t\right)_{x}
$$

in $\left(k(y) \otimes_{k_{0}} \mathbb{C}\right)^{\times} / F^{\times H_{0} m_{k_{0}}}(k(y), \mathbb{C})=\left(\mathbb{C}^{\times} / F^{\times}\right)^{\operatorname{Hom}_{k_{0}}(k(y), \mathbb{C})}$. It is clear that the Lemma follows from this.

To prove the equality, we may assume $k=k_{0}=\mathbb{C}$ and we may consider locally in the classical topology by dropping the assumption that $X$ and $Y$ are compact. Hence it is enough to consider the $m$-fold branched covering of the punctured disc $f: \Delta^{*} \rightarrow \Delta^{*}$. Take uniformizers $\pi, \pi^{\prime}$ of $\Delta$ on lower and upper stairs respectively satisfying $\pi=\pi^{\prime m}$. Let $\nabla_{x}=\operatorname{res}_{x} \nabla \log e$, and define holomorphic invertible functions

$$
\psi=\left(\varphi, \pi^{\prime}\right)^{\sim}=(-1)^{-\nabla_{x}} \pi^{\prime \nabla_{x}} \times \varphi
$$

and $u=t \cdot \pi^{-r}$ where $r=\operatorname{ord}_{y} t$ on $\Delta$. Then the right hand side is computed as

$$
N_{x / y}\left(\varphi, f^{*} t\right)_{x}=\left(\varphi, f^{*} t\right)_{x}=\psi(x)^{\operatorname{ord}_{x}\left(f^{*} t\right)} u(y)^{\nabla_{x}}=\psi(x)^{m r} u(y)^{\nabla_{x}} .
$$

We compute the left hand side. By $\nabla \log N e=\operatorname{Tr} \nabla \log e$, we have

$$
\nabla_{y}\left(=\operatorname{res}_{y} \nabla \log N e\right)=\nabla_{x}\left(=\operatorname{res}_{x} \nabla \log e\right) .
$$

Hence

$$
\begin{aligned}
& N \psi=(N(-1))^{-\nabla_{x}}(N \pi)^{\prime \nabla_{x}} \times N \varphi \\
= & (-1)^{-m \nabla_{x}}\left((-1)^{m-1} \pi\right)^{\nabla_{x}} \times N \varphi=(-1)^{-\nabla_{x}} \pi^{\nabla_{y}} \times N \varphi=(N \varphi, \pi)^{\sim}
\end{aligned}
$$

and we obtain

$$
(N \varphi, t)_{y}=N \psi(y)^{r} u(y)^{\nabla_{y}}=\psi(x)^{m r} u(y)^{\nabla_{y}}
$$

and the right hand sides of the equalities (1) and (2) are the same. Thus the Lemma is proved.

Now we assume $k=k_{0}, X=\mathbb{P}_{k}^{1}$ and $f=t$ is the coordinate. We prove the identity $(\mathcal{M}, \partial t)_{X}=1 \in M \operatorname{Pic}_{k_{0}, F}(k)$ for an object $\mathcal{M}=((\mathcal{E}, \nabla), V, \rho)$ in $P_{k_{0}, F, X}(U)$. By shrinking $U$ if necessary, we may assume $0, \infty \in D$ and we may take a basis $e$ of $\mathcal{E}$ on $U$. We consider an extension $\mathcal{E}_{X}=\mathcal{O}_{X} \cdot e$. It is enough to show that $\prod_{x} N_{x / k}(\varphi, f)_{x}$ is contained in $F^{\times} \subset \mathbb{C}^{\times}$where $\varphi=\rho(v) / e$ as in the description of the tame symbol. Since we have chosen a $k$-rational base $e$ of $\mathcal{E}$, we may assume $k=\mathbb{C}$.

We take a simply connected path $\gamma$ connecting 0 to $\infty$ which does not pass other points in $D$. Let $|\gamma|$ denote the underlying set and put $|\gamma|^{*}=|\gamma|-\{0, \infty\}$. We take a small tubular neighborhood $T$ of $|\gamma|^{*}$. There are 2 connected components $T^{+}$ and $T^{-}$of $T-|\gamma|^{*}$. We assume that a small circle around 0 with positive direction cuts $\gamma$ from $T^{-}$to $T^{+}$. We take a branch $\log t$ of the logarithm of the coordinate $t$ 
on the simply connected region $\mathbb{P}_{\mathbb{C}}^{1}-|\gamma|$. Let $\log ^{+} t$ and $\log ^{-} t=\log ^{+} t+2 \pi \sqrt{-1}$ be the branch of the logarithm on $|\gamma|^{*}$ continuous on $|\gamma|^{*} \cup T^{+}$and $|\gamma|^{*} \cup T^{-}$ respectively. Take a basis $v$ of the $F$-vector space $V\left(|\gamma|^{*}\right)$ of dimension 1 . We define a basis $\left(v, \partial_{x} t\right)$ of $\left(V, \partial_{x} t\right)$ for $x \in D$. For $x \neq 0, \infty$, let $(v, t)=t^{\nabla_{x}}$ be the section $\exp \left(\nabla_{x} \cdot \log t\right)$ of $(V, t)^{\sim}=\mathcal{O}_{X}^{a n}$ and $\left(v, \partial_{x} t\right)$ be its fiber at $x$. For $x=0$, let $(v, t)=(-1)^{-\nabla_{0}} t^{\nabla_{0}} v$ be the section $\exp \left(\nabla_{0}\left(-\pi \sqrt{-1}+\log ^{-} t\right)\right) \cdot v$ of $\left.(V, t)^{\sim}\right|_{|\gamma|^{*}}$ and $\left(v, \partial_{0} t\right)$ be its fiber at 0 . Similarly for $x=\infty$, let $(v, t)=(-1)^{\nabla \infty} t^{\nabla} v^{\otimes-1}$ be the section $\exp \left(\nabla_{\infty}\left(\pi \sqrt{-1}+\log ^{+} t\right)\right) \cdot v^{\otimes-1}$ of $\left.(V, t)^{\sim}\right|_{|\gamma|^{*}}$ and $\left(v, \partial_{\infty} t\right)$ be its fiber at $\infty$.

It is enough to show that

$$
\prod_{x \in D} \frac{\left(\rho, \partial_{x} t\right)\left(v, \partial_{x} t\right)}{\left(e, \partial_{x} t\right)}=1
$$

We compute each term for $x \in D$. For $x \neq 0, \infty$, it is $t(x)^{\nabla_{x}}=\exp \left(\nabla_{x} \log t(x)\right)$. Here $\log$ is the branch chosen above and $\nabla_{x}=\operatorname{res}_{x}(\nabla \log e)$. We take a base point $b$ on $|\gamma|^{*}$. Then for $x=0$ and $\infty$ they are

$$
\begin{gathered}
\exp \left(\nabla_{0}\left(-\pi \sqrt{-1}+\log ^{-} t(b)\right)+\int_{b}^{0}\left(\nabla_{0} d \log t-\nabla \log e\right)\right) \times \frac{\rho(v)(b)}{e(b)}, \\
\exp \left(\nabla_{\infty}\left(\pi \sqrt{-1}+\log ^{+} t(b)\right)+\int_{b}^{\infty}\left(\nabla_{\infty} d \log t+\nabla \log e\right)\right) \times\left(\frac{\rho(v)(b)}{e(b)}\right)^{-1} .
\end{gathered}
$$

Therefore it is sufficient to show

$$
\begin{gathered}
\nabla_{0}\left(\pi \sqrt{-1}+\log ^{+} t(b)\right)+\int_{b}^{0}\left(\nabla_{0} d \log t-\nabla \log e\right) \\
+\nabla_{\infty}\left(\pi \sqrt{-1}+\log ^{+} t(b)\right)+\int_{b}^{\infty}\left(\nabla_{\infty} d \log t+\nabla \log e\right) \\
+\sum_{x \in D, \neq 0, \infty} \nabla_{x} \cdot \log t(x)=0 .
\end{gathered}
$$

For $x \in D, \neq 0, \infty$, let $\gamma_{x}$ be a small circle with positive direction around $x$. For $x=0$, let $\gamma_{0}$ be a loop starting at $b$, going along $\gamma$ in $T^{+}$, turning around 0 in the positive direction and going back to $b$ along $\gamma$ in $T^{-}$. Similarly for $x=\infty$, let $\gamma_{\infty}$ be a loop starting at $b$, going along $\gamma$ in $T^{-}$, turning around $\infty$ in the positive direction and going back to $b$ along $\gamma$ in $T^{+}$. Then by Cauchy's integral theorem, the sum of the integral of the holomorphic 1 -form

$$
\sum_{x \in D}\left(\frac{1}{2 \pi \sqrt{-1}} \int_{\gamma_{x}} \log t \nabla \log e\right)=0
$$

Hence it is enough to show that the integrals are equal to the corresponding terms in the equality above. For $x \in D, \neq 0, \infty$, it is the theorem of residue. For 0 and $\infty$, we have

$$
\frac{1}{2 \pi \sqrt{-1}} \int_{\gamma_{0}} \log t d \log t=-\frac{1}{2 \pi \sqrt{-1}} \int_{\gamma_{\infty}} \log t d \log t=\log ^{+} t(b)+\pi \sqrt{-1} .
$$

Since $\nabla \log e-\nabla_{0} d \log t$ is holomorphic at 0 , we have

$$
\frac{1}{2 \pi \sqrt{-1}} \int_{\gamma_{0}} \log t\left(\nabla \log e-\nabla_{0} d \log t\right)=\int_{b}^{0} \nabla_{0} d \log t-\nabla \log e .
$$


Similarly for $\infty$, we have

$$
\frac{1}{2 \pi \sqrt{-1}} \int_{\gamma_{\infty}} \log t\left(\nabla \log e+\nabla_{\infty} d \log t\right)=\int_{b}^{\infty} \nabla \log e+\nabla_{0} d \log t .
$$

Thus Proposition 2 is proved for $X=\mathbb{P}_{k}^{1}$ and hence for curves.

(c) Higher-dimensional case. We prove Proposition 2 for higher dimensions. First we consider the case $f \in \bigoplus_{i \in I_{y}} K_{2}(y)=\operatorname{Ker}\left(B_{y} \rightarrow \kappa(y)^{\times}\right)$. Let $D_{i}$ be an irreducible component of $D$ and $k_{i}$ be the constant field of $D_{i}$. We define a pairing

$$
(,)_{i}: M P i c_{k_{0}, F, X}(U) \times k_{i}^{\times} \rightarrow M P i c_{k_{0}, F}\left(k_{i}\right)
$$

by putting $(\mathcal{M}, u)=u^{\mathrm{res}_{i} \nabla \log e}$ where $\operatorname{res}_{i} \nabla \log e \in k_{i}$ is the residue of the logarithmic 1-form $\nabla \log e$ for a local basis $e$ of $\mathcal{E}$. It is independent of the choice of the basis $e$ and the pairing is well-defined.

For $x \in X_{0}$, the restriction of the pairing $N_{x / k} \circ(,)_{x}: M P i c_{k_{0}, F, X}(U) \times$ $A_{x} \rightarrow M P i c_{k_{0}, F}(k)$ to the $i$-component $\kappa(x)^{\times} \subset A_{x}$ is identified with the pairing $N_{k_{i} / k} \circ\left(, N_{x / k_{i}}()\right)_{i}$ for $i \in I_{x}$. On $\bigoplus_{i \in I_{y}} K_{2}(y)=\operatorname{Ker}\left(B_{y} \rightarrow \kappa(y)^{\times}\right)$, the map $\partial_{x, y}: B_{y} \rightarrow A_{x}$ is the direct sum of the tame symbol $\partial_{x}: K_{2}(y) \rightarrow \kappa(x)^{\times}$. Hence the restriction of the pairing $(, \partial)_{X}: P_{k_{0}, F, X}(U) \times B_{y} \rightarrow P_{k_{0}, F}(k)$ to the $i$-component $K_{2}(y) \subset B_{y}$ for $i \in I_{y}$ is identified with the pairing $N_{k_{i} / k} \circ\left(, \prod_{x \in Y} N_{x / k_{i}}\left(\partial_{x}\right)\right)_{i}$ where $Y$ is the closure of $\{y\}$. Hence by the reciprocity law $1=\prod_{x \in Y} N_{x / k_{i}}\left(\partial_{x}\right)$ : $K_{2}(y) \rightarrow k_{i}^{\times}$, the restriction of the pairing $(, \partial)_{X}$ to $\operatorname{Ker}\left(B_{y} \rightarrow \kappa(y)^{\times}\right)$is trivial.

We prove the general case by reducing to a curve. The assertion has been proved for an element in the kernel of $B_{y} \rightarrow \kappa(y)^{\times}$for a point $y \in X_{1}$. Hence it is sufficient to show that, for $f \in \kappa(y)^{\times}$, there exists an element $\tilde{f} \in B_{y}$ lifting $f$ such that $(, \partial \tilde{f})_{X}$ is identical to one. A lifting $\tilde{f}$ may be constructed by taking a family $\pi=\left(\pi_{i}\right)_{i \in I_{y}}$ of local bases of $\mathcal{O}_{X}\left(-D_{i}\right)$. In fact, if $\pi$ denotes its class in $A_{y}$, the image of the product $\tilde{f}=\{\pi, f\} \in B_{y}$ in $\kappa(y)^{\times}$is $f$ since $\operatorname{ord}_{y} \pi=1$. Let $Y$ be the closure of $\{y\}$ and $W$ be an open subscheme of $Y$ such that on a neighborhood of $W, \pi_{i}$ defines a basis of $\mathcal{O}_{X}\left(-D_{i}\right)$. We compute $(\mathcal{M}, \partial\{\pi, f\})_{X}$ using tame symbols and will show $(\mathcal{M}, \partial\{\pi, f\})_{X}=1$ in $M P i c_{k_{0}, F}(k)$ for a suitable choice of $\pi$.

Let $\tilde{Y}$ be the normalization of $Y, \tilde{W}$ be the inverse image of $W$ in $\tilde{Y}$ and $\tilde{W}^{*}$ be the inverse image of $W-\bigcup_{i \notin I_{y}} D_{i}$. For an object $\mathcal{M}$ of $P_{k_{0}, F, X}(U)$, we define an object $(\mathcal{M}, \pi)$ of $P_{k_{0}, F}\left(\tilde{W}^{*}\right)$ as follows. The construction is quite similar to that in the definition of the tame symbol and it is in fact possible to give a unified treatment. We take an extension $\mathcal{E}_{X}$ of $\mathcal{E}$. It is an invertible $\mathcal{O}_{X}$-module. Let $\nabla_{i}=\operatorname{res}_{i}\left(\nabla: \mathcal{E}_{X}\right) \in k_{i}$. We define an integrable connection $(\nabla, \pi)^{\sim}$ on $\mathcal{E}_{X}$ by

$$
(\nabla, \pi)^{\sim} \log e=\nabla \log e-\sum_{i} \nabla_{i} d \log \pi_{i}
$$

on an etale neighborhood $\tilde{X} \supset W$. The residues for $i \in I_{y}$ of the connection $(\nabla, \pi)^{\sim}$ are 0 and hence the connection is defined in a neighborhood of $W-\bigcup_{i \notin I_{y}} D_{i}$. By pulling back, we obtain an integrable connection $((\mathcal{E}, \pi),(\nabla, \pi))$ on $\tilde{W}^{*}$. We apply a similar construction for the local systems. Let $(V, \pi)^{\sim}$ be the subsheaf of $\mathcal{E}_{X}^{a n}$ defined by

$$
(V, \pi)^{\sim}=(-1)^{-\nabla} \pi^{\nabla} \rho(V),
$$

where $(-1)^{-\nabla} \pi^{\nabla}=\exp \left(\sum_{i} \nabla_{i}\left(-\pi \sqrt{-1}+\log \pi_{i}\right)\right)$ as before. The local system $(V, \pi)^{\sim} \otimes \mathbb{C}$ is equal to the sheaf of horizontal sections of $(\nabla, \pi)^{\sim, a n}$. Since the 
residues are 0 , the sheaf $(V, \pi)^{\sim}$ is unramified along $D_{i}^{a n}$ for $i \notin I_{y}$ and is extended to an neighborhood of $W^{a n}-\bigcup_{i \notin I_{y}} D_{i}^{a n}$ as a local system of $F$-vector spaces of rank 1. By pulling back, we obtain a local system of $F$-vector spaces $(V, \pi)$ on $\tilde{W}^{* a n}$. The inclusion $(V, \pi)^{\sim} \rightarrow \mathcal{E}^{a n}$ induces a comparison morphism $(\rho, \pi):(V, \pi) \rightarrow(\mathcal{E}, \pi)^{a n}$. Thus a triple $(\mathcal{M}, \pi)=(((\mathcal{E}, \pi),(\nabla, \pi)),(V, \pi),(\rho, \pi))$ is defined.

To compute $(\mathcal{M},\{\pi, f\})_{X}$, we define $\left(\mathcal{M}, \partial_{\tilde{x}} \tilde{f}\right)_{\tilde{x}} \in M P i c_{k_{0}, F}(\tilde{x})$ for a closed point $\tilde{x} \in \tilde{Y}$. It is enough to define a pairing $(,)_{\tilde{x}}: M P i c_{k_{0}, F}(U) \times A_{\tilde{x}} \rightarrow M P i c_{k_{0}, F}(\tilde{x})$. (The group $A_{\tilde{x}}$ is defined in Section 2(b), just before defining the boundary map $\left.\partial_{x, y}.\right)$ Since $A_{\tilde{x}}$ is the pushout of the diagram

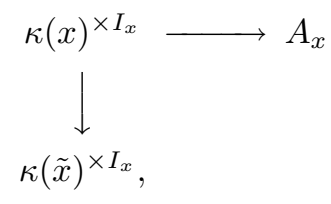

for the image $x \in X$ of $\tilde{x}$, it is enough to define a pairing $(,)_{\tilde{x}}: M P i c_{k_{0}, F}(U) \times$ $\kappa(\tilde{x})^{\times I_{x}} \rightarrow M P i c_{k_{0}, F}(\tilde{x})$ extending the restriction

$$
\begin{aligned}
M \operatorname{Pic}_{k_{0}, F}(U) \times \kappa(x)^{\times I_{x}} & \rightarrow M P i c_{k_{0}, F}(U) \times A_{x} \\
& \stackrel{(,)_{x}}{\longrightarrow} \operatorname{MPic}_{k_{0}, F}(x) \rightarrow M P i c_{k_{0}, F}(\tilde{x}) .
\end{aligned}
$$

It is defined by

$$
(\mathcal{M}, u)_{\tilde{x}}=\prod_{i \in I_{x}} u_{i}^{\operatorname{res}_{i} \nabla \log e} \in M P i c_{k_{0}, F}(\tilde{x})
$$

for $u=\left(u_{i}\right)_{i} \in \kappa(\tilde{x})^{\times I_{x}}$ and a local basis $e$ of the de Rham component $\mathcal{E}$ of $\mathcal{M}=((\mathcal{E}, \nabla), V, \rho) \in P_{k_{0}, F}(U)$.

Now we compute $(\mathcal{M},\{\pi, f\})_{X}$ for a suitable choice of $\pi$. Since we assume $X$ is projective, we may take $\pi$ so that $W$ satisfies the following condition.

$W$ contains the singular points of $Y$, the intersection $Y \cap D_{j}$ for $j \notin I_{y}$ and the zeroes and poles of $f$.

We will take such $\pi=\left(\pi_{i}\right)_{i}$ and show $(\mathcal{M},\{\pi, f\})_{X}=1$ in $M P i c_{k_{0}, F}(k)$ which completes the proof of Proposition 2.

Claim. Assume $\pi$ satisfies the condition above. The triple $(\mathcal{M}, \pi)$ is in $P_{k_{0}, F}\left(\tilde{W}^{*}\right)$, namely, it is regular along $\tilde{x} \in \tilde{Y}$. For a closed point $\tilde{x} \in \tilde{Y}$, we have

$$
\left(\mathcal{M}, \partial_{\tilde{x}} \tilde{f}\right)_{\tilde{x}}=((\mathcal{M}, \pi), f)_{\tilde{x}}
$$

in $M \operatorname{Pic}_{k_{0}, F}(\tilde{x})$.

The Claim will complete the proof of Proposition 2. In fact, for $f \in \kappa(y)^{\times}$, we take $\pi$ as above and then we have

$$
\begin{aligned}
& (\mathcal{M},\{\pi, \partial f\})_{X}=\prod_{\tilde{x}} N_{\tilde{x} / k}(\mathcal{M},\{\pi, f\})_{\tilde{x}} \\
= & \prod_{\tilde{x}} N_{\tilde{x} / k}((\mathcal{M}, \pi), f)_{\tilde{x}}=((\mathcal{M}, \pi), \partial f)_{\tilde{Y}}=1
\end{aligned}
$$

by the Claim and by Proposition 2 for a curve $\tilde{Y}$ already proved. 
Proof of the Claim. The assertion is local at $\tilde{x} \in \tilde{Y}$. Let $\mathcal{M}=((\mathcal{E}, \nabla), V, \rho)$ be an object of $P_{k_{0}, F, X}(U)$. Take bases $e$ of $\mathcal{E}$ and $v$ of $V$ in the intersection of $U$ with a neighborhood of $x$ and let $\varphi=\rho(v) / e$ be an analytic function.

First we consider $\tilde{x} \in \tilde{W}$. By the definition of the connection $(\nabla, \pi)$, it is clearly regular singular at $\tilde{x}$. We show the equality. It is enough to show that

$$
\left(\varphi, \partial_{\tilde{x}} \tilde{f}\right)_{\tilde{x}}=((\varphi, \pi), f)_{\tilde{x}}
$$

modulo $F^{\times}$. Extend the family $\pi=\left(\pi_{i}\right)_{i \in I_{y}}$ to the family $\tilde{\pi}=\left(\pi_{i}\right)_{i \in I_{x}}$ of bases of $\mathcal{O}_{X_{\Sigma}}\left(-D_{i}\right)$ at $x$ as before. By the explicit computation

$$
\partial_{\tilde{x}, y}\{\pi, \bar{f}\}=\tilde{\pi}^{\operatorname{ord}_{\tilde{x}} f} \times\left((-1)^{\operatorname{ord}_{\tilde{x}} f}\left(\pi_{i}, f\right)_{\tilde{x}}^{-1}\right)_{i \in I_{x}-I_{y}},
$$

the left hand side is

$$
\left((-1)^{-\sum_{i \in I_{x}} \operatorname{res}_{i} \nabla \log e} \varphi \cdot \prod_{i \in I_{x}} \pi_{i}^{\operatorname{res}_{i} \nabla \log e}\right)^{\operatorname{ord} \tilde{x} f} \cdot \prod_{i \in I_{x}-I_{y}}\left((-1)^{\operatorname{ord} \tilde{x} f}\left(\pi_{i}, f\right)_{\tilde{x}}^{-1}\right)^{\operatorname{res}_{i} \nabla \log e} .
$$

By $\operatorname{res}_{\tilde{x}} \nabla \log (e, \pi)=\sum_{i \in I_{x}-I_{y}} m_{i} \cdot \operatorname{res}_{i} \nabla \log e$ where $m_{i}=\operatorname{ord}_{\tilde{x}} \pi_{i}$ for $i \in I_{x}-I_{y}$, the right hand side is

$$
\begin{gathered}
(-1)^{-\sum_{i \in I_{x}-I_{y}} m_{i} \operatorname{res}_{i} \nabla \log e \cdot \operatorname{ord}_{\tilde{x}} f} \times\left((-1)^{-\sum_{i \in I_{y}} \operatorname{res}_{i} \nabla \log e} \varphi \cdot \prod_{i \in I_{y}} \pi_{i}^{\operatorname{res}_{i} \nabla \log e}\right)^{\operatorname{ord}_{\tilde{x}} f} \\
\times f^{\sum_{i \in I_{x}-I_{y}} m_{i} \operatorname{res}_{i} \nabla \log e} .
\end{gathered}
$$

Canceling the factors of $\varphi$ and $i \in I_{y}$, the equality follows from the definition of the tame symbol $\pi_{i}^{\operatorname{ord}_{\tilde{x}} f}\left(\pi_{i}, f\right)_{\tilde{x}}^{-1}=(-1)^{-m_{i} \operatorname{ord}_{\tilde{x}} f} f^{m_{i}}$ for $i \in I_{x}-I_{y}$.

We consider $\tilde{x} \notin \tilde{W}$. By the condition on $W$ above, $Y$ is isomorphic to $\tilde{Y}$ and we write $x=\tilde{x}$. Further, by the condition, $f$ is invertible at $x$ and $x \notin D_{j}$ for $j \notin I_{y}$. Let $\pi^{\prime}=\left(\pi_{i}^{\prime}\right)_{i \in I_{y}}$ be a family of bases of $\mathcal{O}_{Y}\left(-D_{i}\right)$ at $x$ and put $u_{i}=\pi_{i} / \pi_{i}^{\prime} \in \kappa(y)^{\times}$. By the definition of the tame symbol, we have

$$
\nabla \log (e, \pi)=\nabla \log \left(e, \pi^{\prime}\right)-\sum_{i \in I_{y}} \operatorname{res}_{i}(\nabla \log e) \cdot d \log u_{i}
$$

for a basis $e$ of $\mathcal{E}$ on the intersection of $U$ with a neighborhood of $x$. Since $\nabla \log \left(e, \pi^{\prime}\right)$ is regular at $x$ and $d \log u_{i}$ has at most a logarithmic pole at $x$, the connection is regular singular at $x$.

To show the equality, by the same argument as in the proof for $\tilde{x} \in \tilde{W}$ above, it is enough to show

$$
\left(\varphi, \partial_{x} \tilde{f}\right)_{x}=((\varphi, \pi), f)_{x}
$$

for $\varphi=\rho(v) / e$ as above. By $\partial_{x, y} f=\pi^{\prime \operatorname{ord}_{x} f} \times\left(\left\{u_{i}, f\right\}_{x}\right)_{i}=\left(f(x)^{-\operatorname{ord}_{x} \pi_{i}}\right)_{i} \in A_{x}$, the left hand side is

$$
f(x)^{-\sum_{i} \operatorname{ord}_{x} \pi_{i} \cdot \operatorname{res}_{i}(\nabla \log e)}=\exp \left(\sum_{i}-\operatorname{ord}_{x} \pi_{i} \cdot \operatorname{res}_{i}(\nabla \log e) \log f(x)\right) .
$$

By $\operatorname{res}_{x} \nabla \log (e, \pi)=-\sum_{i \in I_{y}} \operatorname{res}_{i}(\nabla \log e) \cdot \operatorname{res}_{x} d \log u_{i}=-\sum_{i \in I_{y}} \operatorname{res}_{i}(\nabla \log e) \cdot$ $\operatorname{ord}_{x} \pi_{i}$, the right hand side is the same. Thus the Claim and hence Proposition 2 is proved. 
Remark. We explain the philosophy behind the definition. This remark of heuristic argument is logically independent of the other part of the paper. Let $P(U)$ be the category of a rank-1 object on $U$ and assume that the class group $C l P(U)$ is $H^{1}(U, G)$ for some group $G$. For a set $I$ of indices of irreducible components of a divisor $D$ with simple normal corssings, let $D_{I}=\bigcap_{i \in I} D_{i}$ and $A_{I}$ be an extension of $\mathbb{Z}$ by $\bigoplus_{i \in I} \Gamma\left(D_{I}, \mathbb{G}_{m}\right)$ defined similarly as above. The pairing $C l P(U) \times A_{I} \rightarrow$ $\mathrm{Cl}\left(D_{I}\right)$ defined by the tame symbol should be interpreted by cohomology as follows. We regard $A_{I}$ as a rough approximation of $H_{D_{I}}^{2 d}(X, j ! \mathbb{Z}(d))$ where $d=$ Card $I$ and $j: U \rightarrow X$ is the open immersion. Then the pairing $C l P(U) \times A_{I} \rightarrow C l P\left(D_{I}\right)$ is induced by the cup-product

$$
H^{1}(U, G) \times H_{D_{I}}^{2 d}(X, j ! \mathbb{Z}(d)) \rightarrow H_{D_{I}}^{2 d+1}(X, G(d))
$$

and the Gysin isomorphism $H_{D_{I}}^{2 d+1}(X, G(d)) \simeq H^{1}\left(D_{I}, G\right)=P\left(D_{I}\right)$. When $d=1$, we put $j ! \mathbb{Z}(1)=\operatorname{Ker}\left(\mathbb{G}_{m, X} \rightarrow \mathbb{G}_{m, D}\right)[1]$. Then we get a map $A \rightarrow H_{D}^{2}(X, j ! \mathbb{Z}(1))$. For general $d$, by cup-product, we get a map $\bigotimes_{i \in I} A_{i} \rightarrow H_{D_{I}}^{2 d}(X, j ! \mathbb{Z}(d))$. The fiber product $A_{I}$ approximates the quotient of the tensor product $\bigotimes_{i \in I} A_{i}$ by the subgroup $\left\langle\otimes a_{i} ; \operatorname{ord}_{i}\left(a_{i}\right)=0\right.$ for at least $2 i$ 's $\rangle$.

There is another way to explain this. We consider the case $J=I$. As in [D5], (1.7.10), we should have a functor $P(U) \rightarrow P\left(N_{I}^{*}\right)$. Here $N_{I}^{*}=N_{I}-\bigcup_{i \in I} N_{i}$ is the complement in the normal bundle $N_{I}$ of $D_{I}$ in $X$ of the normal bundles $N_{i}$, $D_{I}$ in $D_{i}$ for $i \in I$. An element $f$ in $A_{I}$ defines a section $\bar{f}: D_{I} \rightarrow N_{I}^{*}$. Then the pairing $(, f): P(U) \rightarrow P\left(D_{I}\right)$ is the composition of the pull-back by $\bar{f}$ with $P(U) \rightarrow P\left(N_{I}^{*}\right)$.

\section{MAin RESUlt}

(a) Statement of the Theorem. Let $k_{0}$ and $F$ be subfields of $\mathbb{C}$ and $U$ be a smooth separated scheme over a finite extension $k$ of $k_{0}$. Let $X$ be a proper smooth scheme over $k$ including $U$ as the complement of a divisor $D$ with simple normal crossings. For an object $\mathcal{M}$ of $M_{k_{0}, F}(U)$ (Definition 2), we have defined the period $\operatorname{per}_{c}(\mathcal{M})$ (Definition 3), the $\Gamma$-factor $\Gamma(\nabla: \mathcal{M})$ (Definition 4) and the tame symbol $\left(\operatorname{det} \mathcal{M}, c_{X \bmod D}\right) \in M P i c_{k_{0}, F}(k)=k^{\times} \backslash\left(k \otimes_{k_{0}} \mathbb{C}\right)^{\times} /\left(F^{\times}\right)^{\text {Hom }_{k_{0}}(k, \mathbb{C})}$ (Definition 6, Definition 7).

Theorem 1. Assume $X$ is projective. Then for an object $\mathcal{M}$ of $M_{k_{0}, F}(U)$, we have an equality

$$
\begin{aligned}
& \operatorname{per}_{c}(\mathcal{M})=\operatorname{per}_{c}(\mathbf{1})^{\operatorname{rank} \mathcal{M}} \times \Gamma(\nabla: \mathcal{M})^{-1} \times\left(\operatorname{det} \mathcal{M}, c_{X \bmod D}\right) \\
& \text { in } \operatorname{MPic}_{k_{0}, F}(k)=k^{\times} \backslash\left(k \otimes_{k_{0}} \mathbb{C}\right)^{\times} /\left(F^{\times}\right)^{\operatorname{Hom}_{k_{0}}(k, \mathbb{C})} .
\end{aligned}
$$

We give a more precise statement, Theorem 3 , giving an isomorphism for a curve $X$ in Section 6. The following equivalent form of Theorem 1, stated in terms of usual cohomology, is that announced in [ST].

Corollary. Under the same assumption, we have an equality

$$
\begin{array}{r}
\operatorname{per}(\mathcal{M}) \cdot \operatorname{per}(\mathbf{1})^{-\operatorname{rank} \mathcal{M}}=\Gamma(-\nabla: \mathcal{M}) \times\left(\operatorname{det} \mathcal{M}, c_{X \bmod D}\right) \\
\text { in } k^{\times} \backslash\left(k \otimes_{k_{0}} \mathbb{C}\right) \times /\left(F^{\times}\right)^{\text {Hom }_{k_{0}}(k, \mathbb{C})} .
\end{array}
$$

Proof of the Corollary. By Theorem 1 for the dual $\mathcal{M}^{*}$ and by Lemma 1.7, we have $\operatorname{per}(\mathcal{M}) \cdot \operatorname{per}(\mathbf{1})^{-\operatorname{rank} \mathcal{M}}=\Gamma\left(\nabla: \mathcal{M}^{*}\right) \times\left(\operatorname{det} \mathcal{M}^{*}, c_{X \bmod D}\right)^{-1}$. Since $\Gamma\left(\nabla: \mathcal{M}^{*}\right)=$ $\Gamma(-\nabla: \mathcal{M})$ and $\operatorname{det} \mathcal{M}^{*}=(\operatorname{det} \mathcal{M})^{\otimes-1}$, the Corollary follows. 
We explain an outline of the proof of Theorem 1. First, we deduce it for $X=\mathbb{P}_{k}^{1}$ from $[\mathrm{T}]$, Theorem 1.2. Then we study the vanishing cycles of a fibration to a curve to prove the Corollary of Lemma 4.6 stated later. Finally we complete the proof by taking a Lefschetz pencil. The following elementary lemma will be repeatedly used in the proof.

Lemma 4.1. Let $E \subset X$ be a smooth divisor such that $D^{\prime}=D \cup E$ has simple normal crossings. Put $U^{\prime}=X-D^{\prime}$ and $E^{*}=E-D$. Let $\mathcal{M}$ be an object of $M_{k_{0}, F, X}(U)$. Then Theorem 1 for two of $\mathcal{M}, \mathcal{M}^{\prime}=\left.\mathcal{M}\right|_{U^{\prime}}$ and $\mathcal{M}^{\prime \prime}=\left.\mathcal{M}\right|_{E^{*}}$ implies that for the rest.

Proof. It is enough to show

$$
\begin{gathered}
\operatorname{per}_{c}(U, \mathcal{M})=\operatorname{per}_{c}\left(U^{\prime}, \mathcal{M}^{\prime}\right) \cdot \operatorname{per}_{c}\left(E^{*}, \mathcal{M}^{\prime \prime}\right) \\
\Gamma(\nabla: \mathcal{M})=\Gamma\left(\nabla: \mathcal{M}^{\prime}\right) \cdot \Gamma\left(\nabla: \mathcal{M}^{\prime \prime}\right) \\
\left(\operatorname{det} \mathcal{M}, c_{X \bmod D}\right)=\left(\operatorname{det} \mathcal{M}^{\prime}, c_{X \bmod D^{\prime}}\right) \cdot\left(\operatorname{det} \mathcal{M}^{\prime \prime}, c_{E \bmod D \cap E}\right) .
\end{gathered}
$$

To show (1), it is enough to define a canonical isomorphism

$$
\operatorname{det} R \Gamma_{c}(U, \mathcal{M}) \simeq \operatorname{det} R \Gamma_{c}\left(U^{\prime},\left.\mathcal{M}\right|_{U^{\prime}}\right) \otimes \operatorname{det} R \Gamma_{c}\left(E^{*},\left.\mathcal{M}\right|_{E^{*}}\right) .
$$

Let $\mathcal{M}=((\mathcal{E}, \nabla), V, \rho)$ and $\left(\mathcal{E}_{X}, \nabla\right)$ be a small extension of $(\mathcal{E}, \nabla)$. Then $\mathcal{E}_{X}(-E)$ is a small extension of $\left(\left.\mathcal{E}\right|_{U^{\prime}}, \nabla\right)$ and $\left.\mathcal{E}_{X}\right|_{E}$ is a small extension of $\left(\left.\mathcal{E}\right|_{E^{*}}, \nabla\right)$. Let $D R_{X}^{\prime}\left(\mathcal{E}_{X}(-E)\right)$ be the de Rham complex $\left(\mathcal{E}_{X}(-E) \otimes \Omega_{X}^{\bullet}\left(\log D^{\prime}\right)\right)$. Then by the exact sequence

$$
0 \rightarrow \Omega_{X}^{1}\left(\log D^{\prime}\right)(-E) \rightarrow \Omega_{X}^{1}(\log D) \rightarrow \Omega_{E}^{1}(\log (D \cap E)) \rightarrow 0,
$$

we have an exact sequence of de Rham complexes

$$
0 \rightarrow D R_{X}^{\prime}\left(\mathcal{E}_{X}(-E)\right) \rightarrow D R_{X}\left(\mathcal{E}_{X}\right) \rightarrow D R_{E}\left(\left.\mathcal{E}_{X}\right|_{E}\right) \rightarrow 0
$$

From this we obtain an isomorphism

$$
\operatorname{det} R \Gamma_{c}(U, \mathcal{M}) \simeq \operatorname{det} R \Gamma_{c}\left(U^{\prime},\left.\mathcal{M}\right|_{U^{\prime}}\right) \otimes \operatorname{det} R \Gamma_{c}\left(E^{*},\left.\mathcal{M}\right|_{E^{*}}\right)
$$

by $[\mathrm{K}-\mathrm{M}]$.

The equality $\Gamma\left(\nabla: \mathcal{E}_{X}\right)=\Gamma\left(\nabla: \mathcal{E}_{X}^{\prime}\right) \Gamma\left(\nabla:\left.\mathcal{E}_{X}\right|_{E}\right)$ is easily checked and hence the equality (2) follows.

We show (3). Applying Corollary 2 of Lemma 2.3 to $(\mathcal{E}, \rho)=\left(\Omega_{X}^{1}(\log D)\right.$, res) and $(\mathcal{F}, \sigma)=\left(\Omega_{X}^{1}\left(\log D^{\prime}\right)\right.$, res $)$, we obtain $c_{X \bmod D}=c_{X \bmod D^{\prime}}+i_{*} c_{E \bmod D \cap E}$ in $C H^{n}(X \bmod D)$. From this and the definition, the equality (3) follows.

(b) Proof for $X=\mathbb{P}_{k}^{1}$. We deduce Theorem 1 for $X=\mathbb{P}_{k}^{1}$ from [T], Theorem 1.2. Though it may seem that we are giving too much detail in the proof, it is absolutely necessary to prove a stronger result, Theorem 3 in Section 6 . First, we give an equivalent reformulation, Theorem $\mathrm{T}$, stated in the terminology of this paper. Then we deduce it from Theorem 1.2 loc.cit.

Let $D$ be a reduced divisor of $X=\mathbb{P}_{k}^{1}$. We may assume $\infty \notin D$, and put $D^{\prime}=D \cup\{\infty\}$ and $U=X-D^{\prime}$. Let $\mathcal{M}=((\mathcal{E}, \nabla), V, \rho)$ be an object of $M_{k_{0}, F, X}(U)$ and $\left(\mathcal{E}_{X}, \nabla\right)$ be an extension of $(\mathcal{E}, \nabla)$ to $X$.

First we compute the de Rham cohomology assuming that $\mathcal{E}_{X}$ is a free $\mathcal{O}_{X^{-}}$ module $E \otimes_{k} \mathcal{O}_{X}$ for $E=\Gamma\left(X, \mathcal{E}_{X}\right)$. By $\Gamma\left(X, \Omega_{X}^{1}\left(\log D^{\prime}\right)\right) \simeq \mathcal{O}_{D}$ and $H^{1}\left(X, \mathcal{O}_{X}\right)=$ 
$H^{1}\left(X, \Omega_{X}^{1}\left(\log D^{\prime}\right)\right)=0$, we have a quasi-isomorphism

$$
\beta: R \Gamma\left(X, D R\left(\mathcal{E}_{X}\right)\right) \simeq\left[\nabla=\bigoplus_{x \in D} \operatorname{res}_{x} \nabla: E \rightarrow E \otimes \mathcal{O}_{D}\right] .
$$

By identifying $E=\mathcal{E}_{X} \otimes \kappa(\infty)$, we obtain an isomorphism also denoted by $\beta$ :

$$
\beta: \operatorname{det} R \Gamma\left(X, D R\left(\mathcal{E}_{X}\right)\right) \simeq\left(\operatorname{det}\left(E \otimes \mathcal{O}_{D}\right)\right)^{\otimes-1} \otimes \operatorname{det}\left(\mathcal{E}_{X} \otimes \kappa(\infty)\right) .
$$

Next we consider the singular cohomology. Let $A=\mathbb{A}_{k}^{1} \subset X$ and $j: U \rightarrow A$ be the open immersion. We compute $R \Gamma\left(A^{a n}, j ! V\right)$ explicitly by choosing some paths. For each embedding $k \rightarrow \mathbb{C}$ over $k_{0}$, we take a base point $b \in U^{a n}$, a small disc $\Delta_{x} \subset X^{a n}$ with the center at $x$ for each $x \in D^{\prime a n}$, a base point $b_{x}$ of the punctured $\operatorname{disc} \Delta_{x}^{*}=\Delta_{x}-\{x\}$ and a path $\gamma_{x}$ connecting $b$ and $b_{x}$. We assume that the union $|\gamma|=\bigcup_{x \in D^{\prime a n}}\left|\gamma_{x}\right|$ of the underlying sets $\left|\gamma_{x}\right|$ of paths $\gamma_{x}$ is contractible. Then we have a distinguished triangle

$$
\rightarrow R \Gamma\left(A^{a n}, j ! V\right) \rightarrow R \Gamma\left(U^{a n}, V\right) \rightarrow \bigoplus_{x \in D^{a n}} R \Gamma\left(\Delta_{x}^{*}, V\right) \rightarrow .
$$

Since the topological space $U^{a n}$ is homotopically equivalent to $\bigcup_{x \in D^{a n}}\left(\left|\gamma_{x}\right| \cup \Delta_{x}^{*}\right)$, we obtain a distinguished triangle

$$
\rightarrow R \Gamma\left(A^{a n}, j_{!} V\right) \rightarrow R \Gamma\left(\bigcup_{x \in D^{a n}}\left|\gamma_{x}\right|, V\right) \rightarrow \bigoplus_{x \in D^{a n}} R \Gamma\left(b_{x}, V\right) \rightarrow
$$

In other word, we have a quasi-isomorphism

$$
\alpha: R \Gamma\left(A^{a n}, j_{!} V\right) \simeq\left[\bigoplus \gamma_{x *}: V_{b} \rightarrow \bigoplus_{x \in D^{a n}} V_{b_{x}}\right] .
$$

Here the isomorphism $\gamma_{x *}: V_{b} \rightarrow V_{b_{x}}$ is defined by the path $\gamma_{x}$. By identifying by the isomorphism $\gamma_{\infty *}: V_{b} \rightarrow V_{b_{\infty}}$, we obtain an isomorphism also denoted by $\alpha$ :

$$
\alpha: \operatorname{det} R \Gamma\left(A^{a n}, j_{!} V\right) \simeq\left(\operatorname{det} \bigoplus_{x \in D^{a n}} V_{b_{x}}\right)^{\otimes-1} \otimes \operatorname{det} V_{b_{\infty}} .
$$

Thirdly, we consider tame symbols. Assuming $k_{0}=k=\mathbb{C}$, we define an isomorphism

$$
(\rho, d t):\left(\left(\operatorname{det} \bigoplus_{x \in D} V_{b_{x}}\right)^{\otimes-1} \otimes \operatorname{det} V_{b_{\infty}}\right) \otimes_{F} \mathbb{C} \rightarrow \operatorname{det}\left(E \otimes \mathcal{O}_{D}\right)^{\otimes-1} \otimes \operatorname{det} \mathcal{E}_{X} \kappa(\infty)
$$

of 1-dimensional $\mathbb{C}$-vector spaces using tame symbols. Since $\operatorname{det} \bigoplus_{x \in D} V_{b_{x}} \simeq$ $\bigotimes_{x \in D} \operatorname{det} V_{b_{x}}$ and $\operatorname{det}\left(E \otimes \mathcal{O}_{D}\right) \simeq \bigotimes_{x \in D} \operatorname{det} \mathcal{E}_{X}(x)$, it is enough to define isomorphisms

$$
\begin{gathered}
(\rho, t-t(x))_{x}: \operatorname{det} V_{b_{x}} \otimes_{F} \mathbb{C} \rightarrow \operatorname{det} \mathcal{E}_{X} \otimes \kappa(x), \\
\left(\rho,-t^{-1}\right)_{\infty}: \operatorname{det} V_{b_{\infty}} \rightarrow \operatorname{det} \mathcal{E}_{X} \otimes \kappa(\infty)
\end{gathered}
$$

for $x \in D$ and $\infty$ and put $(\rho, d t)=\bigotimes_{x}\left(\rho,-t^{-1}\right)_{\infty}$. To simplify the notation, we replace $F$ by $\mathbb{C}$ and $V$ by $\operatorname{det} V$ and assume $F=\mathbb{C}$ and the rank is 1 . For $x \in D$ and $\infty$, let $(\nabla, t-t(x))$ and $\left(\nabla,-t^{-1}\right)$ be the integrable connection on $\mathcal{E}_{X}$ and 
$(V, t-t(x))$ and $\left(V,-t^{-1}\right)$ be the sheaf of their flat sections defined in Section 3 . We define $(\rho, t-t(x))_{x}$ and $\left(\rho,-t^{-1}\right)_{\infty}$ to be the composites

$$
\begin{aligned}
V_{b_{x}} \stackrel{\sim}{\rightarrow}(V, t-t(x))_{b_{x}} & \stackrel{\sim}{\leftarrow} \Gamma\left(\Delta_{x},(V, t-t(x))\right) \stackrel{\sim}{\rightarrow}(V, t-t(x))_{x} \stackrel{\sim}{\rightarrow} \mathcal{E}_{X} \otimes \kappa(x), \\
V_{b_{\infty}} \stackrel{\sim}{\rightarrow}\left(V,-t^{-1}\right)_{b_{\infty}} & \stackrel{\sim}{\leftarrow} \Gamma\left(\Delta_{\infty},\left(V,-t^{-1}\right)\right) \stackrel{\sim}{\rightarrow}\left(V,-t^{-1}\right)_{\infty} \stackrel{\sim}{\rightarrow} \mathcal{E}_{X} \otimes \kappa(\infty) .
\end{aligned}
$$

Here the first arrows are the multiplication by

$$
\begin{aligned}
& (-1)^{\nabla_{x}}\left(t\left(b_{x}\right)-t(x)\right)^{\nabla_{x}}=\exp \left(\left(-\pi \sqrt{-1}+\log \left(t\left(b_{x}\right)-t(x)\right)\right) \nabla_{x}\right) \text { for } x \in D, \\
& (-1)^{\nabla_{\infty}}\left(-t\left(b_{\infty}\right)\right)^{\nabla_{\infty}}=\exp \left(\log t\left(b_{\infty}\right) \nabla_{\infty}\right) \text { for } \infty
\end{aligned}
$$

with the normalization of the branches of logarithm as follows. We choose the branch of the $\operatorname{logarithm} \log (t-t(x))$ for $x \in D$ and $\log t$ on $|\gamma|$ so that

$$
\log \left(t\left(b_{\infty}\right)-t(x)\right)-\log \left(t\left(b_{\infty}\right)\right)=-\sum_{n=1}^{\infty}\left(t(x) / t\left(b_{\infty}\right)\right)^{n} / n
$$

where $|\gamma|$ is as above.

If the extension $\mathcal{E}_{X}$ is small at $D$ and big at $\infty$, the map $\rho$ induces a quasiisomorphism by Lemma 1.6:

$$
R \Gamma(\rho): R \Gamma\left(A^{a n}, j_{!} V\right) \rightarrow R \Gamma\left(X, D R\left(\mathcal{E}_{X}\right)\right) .
$$

Theorem T. Assume $k_{0}=k=\mathbb{C}, \mathcal{E}_{X} \simeq \mathcal{O}_{X}^{r}$ and the real parts of eigenvalues of $\nabla_{x}=\operatorname{res}_{x} \nabla$ are positive for $x \in D$ and negative for $x=\infty$. Put $(-1)^{\operatorname{Tr}\left(\nabla_{\infty}\right)}=$ $\exp \left(\operatorname{Tr}\left(\nabla_{\infty}\right) \pi \sqrt{-1}\right)$ and $\Gamma=\prod_{x \in D} \Gamma\left(\operatorname{res}_{x} \nabla\right) \cdot(-1)^{\operatorname{Tr}\left(\nabla_{\infty}\right)} \cdot \Gamma\left(1-\operatorname{res}_{\infty} \nabla\right)^{-1}$. Then the diagram

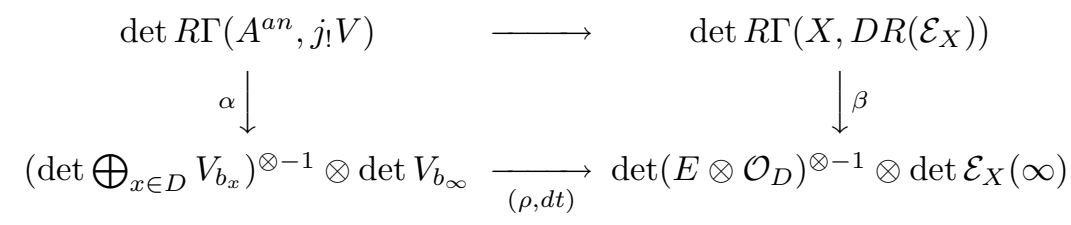

is commutative. Here the upper horizontal arrow is $(-1)^{r(d-1)} \Gamma^{-1}$-times $\operatorname{det} R \Gamma(\rho)$ and $r=\operatorname{rank} \mathcal{M}$ and $d=\operatorname{deg} D$.

Remark. The isomorphisms $\alpha$ and $(\rho, d t)$ may depend on the paths $\left\{\gamma_{i}\right\}_{i}$, but the composite is independent.

Proof. We may assume $F=\mathbb{C}$. We show that the statement is equivalent to Theorem 1.2 of $[\mathrm{T}]$. We define isomorphisms

$$
\begin{gathered}
\left(\bigotimes_{x \in D} \operatorname{det} E\right)^{\otimes-1} \otimes \operatorname{det} E \simeq\left(\operatorname{det} \bigoplus_{x \in D} V_{b_{x}}\right)^{\otimes-1} \otimes \operatorname{det} V_{b_{\infty}} \\
\left(\bigotimes_{x \in D} \operatorname{det} E\right)^{\otimes-1} \otimes \operatorname{det} E \simeq \operatorname{det}\left(E \otimes \mathcal{O}_{D}\right)^{\otimes-1} \otimes \operatorname{det} \mathcal{E}_{X}(\infty)
\end{gathered}
$$

as follows. The second isomorphism is defined by the canonical isomorphism $E \rightarrow$ $\mathcal{E}_{X} \otimes \kappa(x)$ for $x \in D^{\prime}$. The first one is induced by the composition of the canonical isomorphisms $E \rightarrow \mathcal{E}_{X} \otimes \kappa(b), \rho(b): \mathcal{E}_{X} \otimes \kappa(b) \rightarrow V_{b}$ and $\gamma_{x *}: V_{b} \rightarrow V_{b_{x}}$ for $x \in D^{\prime}$. By the isomorphisms, the maps $\beta \circ \operatorname{det} R \Gamma(\rho) \circ \alpha^{-1}$ and $(\rho, d t)$ are regarded as the 
multiplications by numbers. Let $\operatorname{det} R \Gamma(\rho)$ and $(\rho, d t)$ also denote the corresponding numbers respectively. We prove

$$
\begin{aligned}
(-1)^{r(\operatorname{deg} D-1)} \operatorname{det} R \Gamma(\rho) \times \operatorname{det}\left(-\operatorname{res}_{\infty} \nabla\right) & =\operatorname{det} H_{c}, \\
(-1)^{-\operatorname{Tr}\left(\nabla_{\infty}\right)} \Gamma \times \operatorname{det}\left(-\operatorname{res}_{\infty} \nabla\right) & =\prod_{i=1}^{n} \Gamma_{\lambda_{i}}(P) \Gamma_{\infty}(-P)^{-1}, \\
(-1)^{\operatorname{Tr}\left(\nabla_{\infty}\right)}(\rho, d t) & =\prod_{i=1}^{n}\left(P, x-\lambda_{i}\right)_{\gamma_{i}} \times(P, 1 / x)_{\gamma_{\infty}}^{-1} .
\end{aligned}
$$

Here the notation in the right hand sides is that in Theorem 1.2 loc.cit. The equalities imply that the statements are equivalent.

We give a brief correspondence between the notation here and loc.cit. The logarithmic integrable connection $\nabla: \mathcal{E}_{X} \rightarrow \mathcal{E}_{X} \otimes \Omega_{X}^{1}(\log D)$ here corresponds to $\partial_{P}: \mathcal{O}\left(V^{*}\right)_{\log } \rightarrow \Omega^{1}\left(V^{*}\right)_{\log }$ defined by $\partial_{P} f=d f+f P$ in Section 1.2 loc.cit. The global section $E=\Gamma\left(X, \mathcal{E}_{X}\right)$ here is $V^{*}$ there. The coordinate $t$ here is denoted by $x$ there and the value $t\left(x_{i}\right)$ for $x_{i} \in D$ is $\lambda_{i} \in \mathbb{C}$ there. The residues $\nabla_{i}=\operatorname{res}_{x_{i}} \nabla$ at $x_{i} \in D$ here is the right multiplication by the matrix $B^{(i)}: V^{*} \rightarrow V^{*}$.

We reformulate the definition of $\operatorname{det} H_{c}$. Fix a numbering $D=\left\{x_{1}, \ldots, x_{n}\right\}$. We define isomorphisms $E^{n-1} \simeq H^{1}\left(X, D R\left(\mathcal{E}_{X}\right)\right)$ and $E^{* n-1} \simeq H^{1}\left(A^{a n}, j_{!} V\right)^{*}$. Here * denotes the dual. For de Rham cohomology, we have defined a quasi-isomorphism

$$
\beta: R \Gamma\left(X, D R\left(\mathcal{E}_{X}\right)\right) \simeq\left[\bigoplus_{x} \operatorname{res}_{x} \nabla: E \rightarrow \bigoplus_{x \in D} E\right] .
$$

Since $\sum_{x \in D} \operatorname{res}_{x} \nabla=-\operatorname{res}_{\infty} \nabla: E \rightarrow E$ is an isomorphism, sum : $\bigoplus_{x \in D} E \rightarrow E$ induces a retraction of $\bigoplus_{x} \operatorname{res}_{x} \nabla: E \rightarrow \bigoplus_{x \in D} E$. Hence $H^{q}\left(X, D R\left(\mathcal{E}_{X}\right)\right)=0$ for $q \neq 1$ and an isomorphism $\beta^{\prime}: H^{1}\left(X, D R\left(\mathcal{E}_{X}\right)\right) \rightarrow \operatorname{Ker}\left(\operatorname{sum}: \bigoplus_{x \in D} E \rightarrow E\right)$. For singular cohomology, we have

$$
\left.\alpha: R \Gamma\left(A^{a n}, j_{!} V\right) \simeq\left[\bigoplus_{x} \gamma_{x *}: V_{b} \rightarrow \bigoplus_{x \in D} V_{b_{x}}\right] \simeq \text { diagonal }: E \rightarrow \bigoplus_{x \in D} E\right] .
$$

Hence we have $H^{q}\left(A^{a n}, j ! V\right)=0$ for $q \neq 1$ and

$$
\left.H^{1}\left(A^{a n}, j_{!} V\right)=\text { Coker(diagonal }: E \rightarrow \bigoplus_{x \in D} E\right) .
$$

Taking the dual, we obtain an isomorphism

$$
\alpha^{*}: H^{1}\left(A^{a n}, j_{!} V\right)^{*}=\operatorname{Ker}\left(\operatorname{sum}: \bigoplus_{x \in D} E^{*} \rightarrow E^{*}\right) .
$$

We identify $E^{n-1} \simeq \operatorname{Ker}\left(\operatorname{sum}: E^{n} \rightarrow E\right.$ ) by $i d \times 0-0 \times i d$. Here $i d \times 0$ : $E^{n-1} \rightarrow E^{n-1} \times E=E^{n}$ and $0 \times i d: E^{n-1} \rightarrow E \times E^{n-1}=E^{n}$. Similarly we identify $E^{* n-1} \simeq \operatorname{Ker}\left(\operatorname{sum}: E^{* n} \rightarrow E^{*}\right)$ by $i d \times 0-0 \times i d$. Thus we have defined isomorphisms $E^{n-1} \simeq H^{1}\left(X, D R\left(\mathcal{E}_{X}\right)\right)$ and $E^{* n-1} \simeq H^{1}\left(A^{a n}, j_{!} V\right)^{*}$. The isomorphism $H^{1}(\rho): H^{1}\left(A^{a n}, j_{!} V\right)^{*} \rightarrow H^{1}\left(X, D R\left(\mathcal{E}_{X}\right)\right)$ induces a perfect pairing

$$
\begin{aligned}
& E^{* n-1} \times E^{n-1} \simeq H^{1}\left(A^{a n}, j_{!} V\right)^{*} \times H^{1}\left(X, D R\left(\mathcal{E}_{X}\right)\right) \\
& \stackrel{i d \times H^{1}(\rho)^{-1}}{\longrightarrow} H^{1}\left(A^{a n}, j ! V\right)^{*} \times H^{1}\left(A^{a n}, j ! V\right) \stackrel{\text { can }}{\longrightarrow} \mathbb{C} .
\end{aligned}
$$

Then the determinant $\operatorname{det} H_{c}$ is characterized by the condition that the induced pairing $\operatorname{det}\left(E^{* n-1}\right) \times \operatorname{det}\left(E^{n-1}\right) \rightarrow \mathbb{C}$ is $\operatorname{det} H_{c^{-}}$times the canonical pairing. 
We prove the equality (1). The definition of $\operatorname{det} H_{c}$ above is rephrased as follows. We have quasi-isomorphisms

$$
\begin{aligned}
\beta^{\prime}: R \Gamma\left(X, D R\left(\mathcal{E}_{X}\right)\right) & \simeq\left[\operatorname{sum}: \bigoplus_{x \in D} E \rightarrow E\right], \\
\alpha^{*}: R \Gamma\left(A^{a n}, j_{!} V\right)^{*} & \simeq\left[\operatorname{sum}: \bigoplus_{x \in D} E^{*} \rightarrow E^{*}\right] .
\end{aligned}
$$

They induce isomorphisms

$$
\begin{aligned}
\beta^{\prime}: \operatorname{det} R \Gamma\left(X, D R\left(\mathcal{E}_{X}\right)\right) & \simeq \operatorname{det} E^{\otimes 1-n} \\
\alpha^{*}: \operatorname{det} R \Gamma\left(A^{a n}, j_{!} V\right)^{*} & \simeq \operatorname{det} E^{* \otimes 1-n} .
\end{aligned}
$$

The isomorphism $R \Gamma(\rho): R \Gamma\left(A^{a n}, j_{!} V\right) \rightarrow R \Gamma\left(X, D R\left(\mathcal{E}_{X}\right)\right)$ induces a perfect pairing

$$
\begin{gathered}
\left(\operatorname{det} E^{*}\right)^{\otimes 1-n} \times(\operatorname{det} E)^{\otimes 1-n} \simeq \operatorname{det} R \Gamma\left(A^{a n}, j_{!} V\right)^{*} \times \operatorname{det} R \Gamma\left(X, D R\left(\mathcal{E}_{X}\right)\right) \\
\stackrel{i d \times \operatorname{det} R \Gamma(\rho)^{-1}}{\longrightarrow} \operatorname{det} R \Gamma\left(A^{a n}, j_{!} V\right)^{*} \times \operatorname{det} R \Gamma\left(A^{a n}, j_{!} V\right) \stackrel{\text { can }}{\longrightarrow} \mathbb{C} .
\end{gathered}
$$

Then $\operatorname{det} H_{c}$ is characterized by the condition that the pairing is $\left(\operatorname{det} H_{c}\right)^{-1}$-times the canonical one. Since the isomorphism $\alpha^{*}: \operatorname{det} R \Gamma\left(A^{a n}, j_{!} V\right)^{*} \simeq \operatorname{det} E^{* \otimes 1-n}$ is the dual of $\alpha: \operatorname{det} R \Gamma\left(A^{a n}, j_{!} V\right) \simeq \operatorname{det} E^{\otimes 1-n}$, we have

$$
\operatorname{det} H_{c}=\beta^{\prime} \circ \operatorname{det} R \Gamma(\rho) \circ \alpha^{-1}=\left(\beta^{\prime} \circ \beta^{-1}\right) \operatorname{det} R \Gamma(\rho) .
$$

Hence the equality (1) will follow from $\beta^{\prime} \circ \beta^{-1}=(-1)^{r(n-1)} \operatorname{det}\left(-\operatorname{res}_{\infty} \nabla\right)$. This is the consequence of the Lemma below applied to $E^{\prime}=E^{n}, r=\bigoplus_{x \in D} \operatorname{res}_{x} \nabla$ and $s=$ sum.

Lemma 4.2. Let $r: E \rightarrow E^{\prime}$ and $s: E^{\prime} \rightarrow E$ be homomorphisms of vector spaces $E$ and $E^{\prime}$ of finite dimensions such that $s \circ r$ is an automorphism of E. Consider the quasi-isomorphism $b$

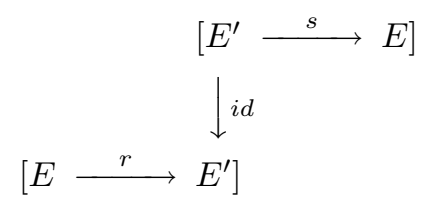

of complexes where $E^{\prime}$ is put on degree 1. Then the automorphism $\operatorname{det} b$ of $\operatorname{det} E \otimes$ $\left(\operatorname{det} E^{\prime}\right)^{\otimes-1}$ is the multiplication by $(-1)^{\operatorname{dim} E\left(\operatorname{dim} E^{\prime}-\operatorname{dim} E\right)} \times \operatorname{det}(s \circ r)^{-1}$.

Proof. Since the determinant of $(s \circ r, i d):\left[E \stackrel{r}{\rightarrow} E^{\prime}\right] \rightarrow\left[E \stackrel{r(s \circ r)^{-1}}{\rightarrow} E^{\prime}\right]$ is det $s \circ r$, we may assume $s \circ r=1$. Then the assertion follows from Koszul's rule on the sign.

The equality (2) follows immediately from the definition, the dictionary $\operatorname{res}_{x_{i}} \nabla=$ $B^{(i)}$ and from the formula $(-s) \Gamma(1-s)=\Gamma(-s)$.

We prove the equality (3). By taking the determinant, we may assume the rank $r$ is 1 . By identification $E \simeq \mathcal{E}_{X}(b) \stackrel{\rho_{b}}{\rightarrow} V_{b} \stackrel{\gamma_{x *}}{\rightarrow} V_{b_{x}}$ and $E \simeq \mathcal{E}_{X}(x)$ for $x \in D^{\prime}$ above, we regard $(\rho, t-t(x))_{x}$ for $x \in D,\left(\rho,-t^{-1}\right)_{\infty}$ etc. as numbers. By the definition $(\rho, d t)=\prod_{x}(\rho, t-t(x))_{x}^{-1} \times\left(\rho,-t^{-1}\right)_{\infty}$ and by the residue formula $\sum_{x \in D, \infty} \nabla_{x}=0$, 
it is sufficient to show that

$$
\begin{gathered}
\left(\rho, t-t\left(x_{i}\right)\right)_{x_{i}}=(-1)^{-\nabla_{x}}\left(P, x-\lambda_{i}\right)_{\gamma_{i}}^{-1}, \\
\left(\rho,-t^{-1}\right)_{\infty}=(P, 1 / x)_{\gamma_{\infty}}^{-1}
\end{gathered}
$$

for $x_{i} \in D$ and $\infty$. We compute the left hand sides following the definition. For $x \in D,=\infty$ and $e \in E$, let $v$ be the (multivalued) section of $V$ on $\Delta_{x}^{*}$ such that stalk $v$ at $b_{x}$ is $\gamma_{x *}\left(\rho_{b}(e)\right)$. Let $\varphi$ be the analytic function $\rho(v) / e$ on $\Delta_{x}^{*}$. Then for $x \in D$ by the definition in Section $3,(\rho, t-t(x))_{x}=(\varphi, t-t(x))_{x}$ is the value at $x$ of the branch of the invertible function $(-1)^{-\nabla_{x}}(t-t(x))^{\nabla_{x}} h$ taking the value $\exp \left(\nabla_{x}\left(-\pi \sqrt{-1}+\log \left(t\left(b_{x}\right)-t(x)\right)\right)\right) \rho(v) / e$ at $b_{x}$. On the other hand, the notation

$$
\left(P, x-\lambda_{i}\right)_{\gamma_{i}}=\lim _{x \rightarrow \lambda_{i}} D(x) \cdot\left(x-\lambda_{i}\right)^{-\operatorname{tr} B^{(i)}}
$$

loc.cit., Section 1.1, Definition, is translated here in the following way. $D(x)=F(x)$ there is $\varphi(x)^{-1}$ here, $\operatorname{tr} B^{(i)}=B^{(i)}$ there is $\nabla_{x_{i}}$ here and $\left(x-\lambda_{i}\right)^{-\operatorname{tr} B^{(i)}}$ there is $\exp \left(-\nabla_{x_{i}} \log \left(t-t\left(x_{i}\right)\right)\right)$. Hence we have

$$
\left(\rho, t-t\left(x_{i}\right)\right)_{x_{i}}=\exp \left(-\nabla_{x_{i}} \pi \sqrt{-1}\right)\left(P, x-\lambda_{i}\right)_{\gamma_{i}}^{-1} .
$$

Similarly $\left(\rho,-t^{-1}\right)_{\infty}=\left(\varphi,-t^{-1}\right)_{\infty}$ is the value at $\infty$ of the branch of the invertible function $t^{-\nabla_{\infty}} \varphi$ taking the value $\exp \left(-\nabla_{\infty} \log \left(t\left(b_{\infty}\right)\right)\right) \rho(v) / e$ at $b_{\infty}$ and $(P, 1 / x)_{\gamma_{\infty}}=\lim _{x \rightarrow \infty} D(x) x^{\operatorname{tr} B^{(\infty)}}$. Hence by a similar dictionary, we have

$$
\left(\rho,-t^{-1}\right)_{\infty}=(P, 1 / x)_{\gamma_{\infty}}^{-1} .
$$

Thus the equality (3) and hence Theorem $\mathrm{T}$ is proved.

Proof of Theorem 1 for $X=\mathbb{P}_{k}^{1}$. Now we deduce Theorem 1 for $X=\mathbb{P}_{k}^{1}$ from Theorem $\mathrm{T}$. We need to eliminate the assumption that $\mathcal{E}_{X}$ is free and to compare $R \Gamma\left(\mathbb{A}^{1},\left.j_{!} V\right|_{U \cap \mathbb{A}^{1}}\right)$ with $R \Gamma_{c}(U, V)$, i.e. to compare $R j_{*}$ with $j_{*}$ at $\infty$.

Since Theorem $\mathrm{T}$ is a statement over $\mathbb{C}$, first we modify it into a more applicable form, Claim 1 below. Changing the coordinate if necessary, we always assume $\infty \in U$. Put $U^{\prime}=U-\{\infty\}$. Let $\mathcal{M}=((\mathcal{E}, \nabla), V, \rho)$ be an object of $M_{k_{0}, F, X}\left(U^{\prime}\right)$. We define a variant of period $\operatorname{per}^{\prime}(\mathcal{M}) \in M P i c_{k_{0}, F}(k)$ as follows. We consider an extension $\mathcal{E}_{X}$ satisfying the condition

(1) $\mathcal{E}_{X}$ is an extension of $\mathcal{E}$ small at $D$ and big at $\infty$.

We obtain a triple

$$
R \Gamma^{\prime}\left(U^{\prime}, \mathcal{M}\right)=\left(R \Gamma\left(X, D R\left(\mathcal{E}_{X}\right)\right), R \Gamma\left(A^{a n}, j_{!} V\right), R \Gamma(\rho)\right) \in M_{k_{0}, F}(k) .
$$

Here the de Rham complex is defined using $\Omega_{X}^{1}(\log D \cup\{\infty\})$. We define

$$
\operatorname{per}^{\prime}(\mathcal{M})=\left[\left(\operatorname{det} R \Gamma\left(X, D R\left(\mathcal{E}_{X}\right)\right), \operatorname{det} R \Gamma\left(A^{a n}, j_{!} V\right), \operatorname{det} R \Gamma(\rho)\right)\right]
$$

where $\mathcal{E}_{X}$ is an extension satisfying the condition (1) above. It is easily checked that the isomorphism class is independent of the choice of $\mathcal{E}_{X}$ provided it satisfies the condition (1).

For an extension $\mathcal{E}_{X}$ of $\mathcal{E}$ satisfying (1), we put

$$
\Gamma^{\prime}\left(\nabla: \mathcal{E}_{X}\right)=\prod_{x \in D} N_{x / k} \Gamma\left(\operatorname{res}_{x} \nabla\right) \times(-1)^{\operatorname{Tr}\left(\nabla_{\infty}\right)} \Gamma\left(1-\operatorname{res}_{\infty} \nabla\right)^{-1} \in\left(k \otimes_{k_{0}} \mathbb{C}\right)^{\times} .
$$

By the equality $s(-1)^{-(s-1)} \Gamma(1-(s-1))=(-1)^{-s} \Gamma(1-s)$, its class $\Gamma^{\prime}(\nabla: \mathcal{M}) \in$ $M P i c_{k_{0}, F}(k)$ is independent of the choice of an extension $\mathcal{E}_{X}$ satisfying (1). 
Theorem $\mathrm{T}$ implies the following.

Claim 1. Assume $\infty \in U \subset \mathbb{P}_{k}^{1}$. Let $\mathcal{M}=((\mathcal{E}, \nabla), V, \rho)$ be an object of $M_{k_{0}, F, X}(U)$. Assume that there exists an extension $\left(\mathcal{E}_{X}, \nabla\right)$ of $(\mathcal{E}, \nabla)$ such that $\mathcal{E}_{X} \simeq \mathcal{O}_{X}(-m)^{r}$ for some $m \in \mathbb{Z}$. Then we have

$$
\operatorname{per}^{\prime}(\mathcal{M})=\operatorname{per}^{\prime}(\mathbf{1})^{r} \cdot \Gamma^{\prime}(\nabla: \mathcal{M})^{-1} \cdot\left(\left.\operatorname{det} \mathcal{M}\right|_{U^{\prime}}, c_{X \bmod D^{\prime}}\right) .
$$

Proof of Claim 1. We assume $\mathcal{E}_{X} \simeq \mathcal{O}_{X}(-m)^{r}$ for some $m$. Replacing $\mathcal{E}_{X}$ by $\mathcal{E}_{X}\left(-m^{\prime} D\right)$ for large $m^{\prime} \in \mathbb{N}$ if necessary, we may assume that

(2) $m>0$ and the real parts of all the conjugates in $\mathbb{C}$ over $k_{0}$ of the eigenvalues of $\operatorname{res}_{x} \nabla \in \kappa(x)$ for $x \in D$ are positive.

We put $\mathcal{E}_{X}^{\prime}=\mathcal{E}_{X}(m[\infty])$. Then $\mathcal{E}_{X}^{\prime} \simeq \mathcal{O}_{X}^{r}$ and $\operatorname{res}_{\infty} \nabla_{\mathcal{E}_{X}^{\prime}}$ is the multiplication by $-m$ and the assumption of Theorem $\mathrm{T}$ is satisfied. The condition is also satisfied for the extension $\mathcal{O}_{X}(-D)$ of $\left(\mathcal{O}_{U}, d\right)$ and $\mathcal{O}_{X}^{\prime}=\mathcal{O}_{X}(-D+\operatorname{deg} D[\infty])$. Now Theorem $\mathrm{T}$ applied to $\mathcal{E}_{X}^{\prime}$ and to $\mathcal{O}_{X}^{\prime}$ implies that

$$
\operatorname{per}^{\prime}(\mathcal{M})=\Gamma^{\prime}(\nabla: \mathcal{M})^{-1}(\operatorname{det} \rho, d t), \quad \operatorname{per}^{\prime}(\mathbf{1})=\Gamma^{\prime}(\nabla: \mathbf{1})^{-1}(\operatorname{can}, d t) .
$$

In fact the isomorphism $\beta$ in Theorem $\mathrm{T}$ is defined over $k$. By the definition of the tame symbol, we have $(\operatorname{det} \rho, d t)=\left(\mathcal{M}, c_{X \bmod D^{\prime}}\right)$. Since $\Gamma^{\prime}(\nabla: \mathbf{1})=(\operatorname{can}, d t)=1$ in $M P i c_{k_{0}, F}^{\prime}(k)$, the Claim is proved.

Finally we complete the proof of Theorem 1 for $\mathbb{P}_{k}^{1}$. We show that the assumption of Claim 1 is not restrictive.

Claim 2. Shrinking $U$ if necessary, there exists an extension $\left(\mathcal{E}_{X}, \nabla\right)$ such that $\mathcal{E}_{X} \simeq \mathcal{O}_{X}(m)^{r}$ for some $m \in \mathbb{Z}$.

Proof of Claim 2. By a theorem of Grothendieck, a locally free $\mathcal{O}_{X}$-module $\mathcal{E}_{X}$ is isomorphic to $\bigoplus_{j} \mathcal{O}_{X}\left(m_{j}\right)$ for some $\left(m_{j}\right)_{j=1, \cdots, r}$. We prove the Claim by induction on $M=\sum_{j}\left(m_{j}-m\right)$ where $m=\min _{j} m_{j}$. If it is 0 , there is nothing to prove. Otherwise, take $j_{0}$ such that $m_{j_{0}} \neq m$ and a $k$-rational point $x \in U$. Then the kernel $\mathcal{E}_{X}^{\prime}$ of the composite $\mathcal{E}_{X} \rightarrow \mathcal{E}_{X}(x) \stackrel{p r_{j_{0}}}{\rightarrow} \mathcal{O}_{X}\left(m_{j_{0}}\right)(x)$ is an extension of $\left.\mathcal{E}\right|_{U-x}$ and $M$ for $\mathcal{E}_{X}^{\prime}$ is $M-1$. Thus Claim 2 is proved.

We show that the conclusion of Claim 1 implies Theorem 1 for $X=\mathbb{P}^{1}$. Let $\mathcal{M}=((\mathcal{E}, \nabla), V, \rho)$ be an object of $M_{k_{0}, F, X}(U)$ and $\mathcal{E}_{X}$ be an extension of $\mathcal{E}$ as in the assumption of Claim 1. It is sufficient to prove the equalities

$$
\begin{gathered}
\operatorname{per}_{c}(\mathcal{M})=\operatorname{per}_{c}\left(U^{\prime},\left.\mathcal{M}\right|_{U^{\prime}}\right) \times\left[\operatorname{det}\left(\left.\mathcal{M}\right|_{\infty}(-1)\right)\right] \\
\Gamma(\nabla: \mathcal{M})=\Gamma^{\prime}(\nabla: \mathcal{M}), \\
\left(\operatorname{det} \mathcal{M}, c_{X \bmod D}\right)=\left(\left.\operatorname{det} \mathcal{M}\right|_{U^{\prime}}, c_{X \bmod D^{\prime}}\right) \times\left[\operatorname{det}\left(\left.\mathcal{M}\right|_{\infty}\right)\right] .
\end{gathered}
$$

The equality (1) follows from the distinguished triangles

$$
\begin{aligned}
& \rightarrow R \Gamma\left(X, D R\left(\mathcal{E}_{X}\right)\right) \rightarrow R \Gamma\left(X, D R^{\prime}\left(\mathcal{E}_{X}\right)\right) \rightarrow \mathcal{E}_{X}(\infty)[-1] \rightarrow \\
& \rightarrow R \Gamma_{c}\left(U^{a n}, V\right) \rightarrow R \Gamma_{c}\left(U^{\prime a n},\left.V\right|_{U^{\prime a n}}\right) \rightarrow V_{\infty}(-1)[-1] \rightarrow .
\end{aligned}
$$

Since $\operatorname{res}_{\infty} \nabla=0$, we have $\Gamma\left(\nabla: \mathcal{E}_{X}\right)=\Gamma^{\prime}\left(\nabla: \mathcal{E}_{X}\right)$ and the equality (2) follows. Finally the equality (3) is a consequence of the formula $c_{X \bmod D}=c_{X \bmod D^{\prime}}+[\infty]$ in Lemma 2.1. Thus the proof of Theorem 2 for $X=\mathbb{P}_{k}^{1}$ is completed. 
(c) Local study of fibration. To prove the general case, we study a fibration $f: X \rightarrow Y$ to a curve $Y$. In this and the next subsections, we keep the following notation.

$\star X$ is a smooth scheme over $k$ and $D=X-U$ is a divisor with simple normal crossings. $Y$ is a smooth curve over $k$ and $E$ is a reduced divisor. $f: X \rightarrow Y$ is a proper flat morphism such that the underlying set of $f^{*}(E)$ is in $D$. On the complement $W=Y-E$, the restriction $f_{W}: X_{W} \rightarrow W$ is smooth and $D_{W}=D \cap X_{W}$ is a divisor with relative normal crossings.

In this subsection, we study the local situation at a closed point $y$ in $E$ and prove Lemma 4.3 stated later.

Let $(\mathcal{E}, \nabla)$ be an integrable connection on $U=X-D$ and $\left(\mathcal{E}_{X}, \nabla\right)$ be a small extension to $X$. Let $\Omega_{X / Y}^{1}(\log D / \log E)$ be the cokernel of the natural morphism $f^{*} \Omega_{Y}^{1}(\log E) \rightarrow \Omega_{X}^{1}(\log D)$. It is a locally free $\mathcal{O}_{X}$-module of $\operatorname{rank} \operatorname{dim} X-1$. Let $\Omega_{X / Y}^{q}(\log D / \log E)=\bigwedge^{q} \Omega_{X / Y}^{1}(\log D / \log E)$. For a logarithmic integrable connection $\left(\mathcal{E}_{X}, \nabla\right)$, we define the relative de Rham complex

$$
\begin{aligned}
& D R_{X / Y}\left(\mathcal{E}_{X}\right)=\left(\mathcal{E}_{X} \otimes \Omega_{X / Y}^{\bullet}(\log D / \log E)\right) \\
= & {\left[\mathcal{E}_{X} \stackrel{\nabla}{\rightarrow} \mathcal{E}_{X} \otimes \Omega_{X / Y}^{1}(\log D / \log E) \stackrel{\nabla}{\rightarrow} \cdots\right] . }
\end{aligned}
$$

On the higher direct image $\mathcal{F}_{Y}^{q}=R^{q} f_{*} D R_{X / Y}\left(\mathcal{E}_{X}\right)$, the Gauss-Manin connection $\nabla: \mathcal{F}_{Y}^{q} \rightarrow \mathcal{F}_{Y}^{q} \otimes \Omega_{Y}^{1}(\log E)$ is defined as follows. By the definition of $D R_{X / Y}\left(\mathcal{E}_{X}\right)$, we have an exact sequence of complexes

$$
0 \rightarrow D R_{X / Y}\left(\mathcal{E}_{X}\right) \otimes f^{*} \Omega_{Y}^{1}(\log E)[-1] \rightarrow D R_{X}\left(\mathcal{E}_{X}\right) \rightarrow D R_{X / Y}\left(\mathcal{E}_{X}\right) \rightarrow 0 .
$$

The connection $\nabla$ is defined as the boundary map of the induced long exact sequence

$$
\rightarrow R^{q} f_{*} D R_{X}\left(\mathcal{E}_{X}\right) \rightarrow \mathcal{F}_{Y}^{q} \stackrel{\nabla}{\rightarrow} \mathcal{F}_{Y}^{q} \otimes \Omega_{Y}^{1}(\log E) \rightarrow .
$$

We generalize the terminology for logarithmic integrable connections on locally free $\mathcal{O}_{Y}$-modules defined in Section 1 to those on coherent modules. Let $(\mathcal{F}, \nabla)$ be an integrable connection on $W$. Let $\left(\mathcal{F}_{Y}, \nabla\right)$ be the pair of a coherent $\mathcal{O}_{Y}$-module $\mathcal{F}_{Y}$ and a logarithmic integrable connection $\nabla: \mathcal{F}_{Y} \rightarrow \mathcal{F}_{Y} \otimes \Omega_{Y}^{1}(\log E)$ extending $(\mathcal{F}, \nabla)$. The connection $\nabla$ induces a connection on the torsion part $\mathcal{F}_{Y, \text { tors }} \rightarrow$ $\mathcal{F}_{Y, \text { tors }} \otimes \Omega_{Y}^{1}(\log E)$. We say an extension $\mathcal{F}_{Y}$ is small if $\mathcal{F}_{Y} / \mathcal{F}_{Y, \text { tors }}$ is small in the sense of Section 1 and if $\mathcal{F}_{Y, \text { tors }} \rightarrow \mathcal{F}_{Y \text {,tors }} \otimes \Omega_{Y}^{1}(\log E)$ is an isomorphism. If $\mathcal{F}_{Y}$ and $\mathcal{F}_{Y}^{\prime}$ are small extensions of $\mathcal{F}$, a morphism $\left(\mathcal{F}_{Y}, \nabla\right) \rightarrow\left(\mathcal{F}_{Y}^{\prime}, \nabla\right)$ extending the identity on $\mathcal{F}$ induces a quasi-isomorphism $D R\left(\mathcal{F}_{Y}\right) \rightarrow D R\left(\mathcal{F}_{Y}^{\prime}\right)$. An extension $\mathcal{F}_{Y}$ is small if and only if $D R\left(\mathcal{F}_{Y}\right)^{a n}=j_{W !}^{a n} D R(\mathcal{F})^{a n}$ by Lemma 1.6.

We define the characteristic rational function $\Phi_{\mathcal{F}_{Y, y}}(T) \in \kappa(y)(T)^{\times}$as follows. The $\kappa(y)$-vector spaces $T_{i}=\operatorname{Tor}_{i}^{\mathcal{O}_{Y, y}}\left(\mathcal{F}_{Y, y}, \kappa(y)\right)$ for $i=0,1$ are the cokernel and the kernel of the map $\mathcal{F}_{Y}(-E)_{y} \rightarrow \mathcal{F}_{Y, y}$ respectively. Hence the connection $\nabla$ induces a $\kappa(y)$-linear endomorphism

$$
\operatorname{res}_{i, y} \nabla: T_{i} \rightarrow T_{i} \otimes \Omega_{Y}^{1}(\log E)_{y} \stackrel{i d \otimes \mathrm{res}_{y}}{\longrightarrow} T_{i} .
$$

We define

$$
\Phi_{\mathcal{F}_{Y, y}}(T)=\prod_{i=0,1} \operatorname{det}\left(T-\operatorname{res}_{i, y} \nabla: \operatorname{Tor}_{i}^{\mathcal{O}_{Y, y}}\left(\mathcal{F}_{Y, y}, \kappa(y)\right)\right)^{(-1)^{i}} .
$$


When $Y$ is proper, we put

$$
\Phi_{\mathcal{F}_{Y}}(T)=\prod_{y \in E} N_{y / k}\left(\Phi_{\mathcal{F}_{Y, y}}(T)\right) \in k(T)^{\times}
$$

and $\Gamma\left(\nabla: \mathcal{F}_{Y}\right)=\left(\Gamma\left(\Phi_{\mathcal{F}_{Y}}(T)\right)\right) \in\left(k \otimes_{k_{0}} \mathbb{C}\right)^{\times}$as in Section 1. For an object $\mathcal{M}=((\mathcal{F}, \nabla), V, \rho)$ of $M_{k_{0}, F, Y}(W)$, the determinant $\operatorname{det}^{\Gamma} R \Gamma_{c}(W, \mathcal{M})$ is canonically isomorphic to the triple

$$
\left(\operatorname{det} R \Gamma\left(Y, D R\left(\mathcal{F}_{Y}\right)\right), \operatorname{det} R \Gamma_{c}(U, V), \Gamma\left(\nabla: \mathcal{F}_{Y}\right)^{-1} \cdot \operatorname{det} R \Gamma_{c}(\rho)\right)
$$

for a small extension $\mathcal{F}_{Y}$ of $\mathcal{F}$.

Lemma 4.3. Let $f: X \rightarrow Y$ and $\left(\mathcal{E}_{X}, \nabla\right)$ be a small extension as above. Then

(1) For each $q$, the Gauss-Manin connection $\left(\mathcal{F}_{Y}^{q}, \nabla\right)$ is small.

(2) For $y \in E$, the characteristic rational functions $\Phi^{q}(T)=\Phi_{\mathcal{F}_{Y}^{q}, y}(T)$ satisfy

$$
\prod_{D_{i} \subset f^{-1}(y)} N_{k_{i} / \kappa(y)}\left(\prod_{\ell=0}^{m_{i}-1} \frac{\Phi_{\mathcal{E}_{X}, i}\left(m_{i} T-\ell\right)}{m_{i}^{\text {rank } \mathcal{E}}}\right)^{c_{i}}=\prod_{q} \Phi^{q}(T)^{(-1)^{q}}
$$

where $k_{i}$ is the constant field of $D_{i}$ and $m_{i}$ is the multiplicity of $D_{i}$ in $f^{-1}(y)$.

Proof. (1) It is sufficient to show that $D R\left(\mathcal{F}_{Y}^{q}\right)^{a n} \simeq j_{W !}^{a n} D R\left(\mathcal{F}^{q}\right)^{a n}$. By the long exact sequence

$$
\rightarrow R^{q} f_{*} D R_{X}\left(\mathcal{E}_{X}\right) \rightarrow \mathcal{F}_{Y}^{q} \rightarrow \mathcal{F}_{Y}^{q} \otimes \Omega_{Y}^{1}(\log E) \rightarrow
$$

and

$$
R^{q} f_{*} D R_{X}\left(\mathcal{E}_{X}\right)^{a n} \simeq R^{q} f_{*} j_{!} D R_{U}(\mathcal{E})^{a n} \simeq j_{W !} D R_{W}\left(\mathcal{F}^{q}\right)^{a n},
$$

we see $\left.D R\left(\mathcal{F}_{Y}^{q}\right)^{a n}\right|_{E^{a n}}=0$. Thus the assertion is proved.

(2) By the exact sequence

$$
0 \rightarrow \mathcal{F}_{Y}^{q} \otimes \kappa(y) \rightarrow H^{q}\left(X_{y}, D R_{X / Y}\left(\mathcal{E}_{X}\right)\right) \rightarrow \operatorname{Tor}_{1}^{\mathcal{O}_{Y}}\left(\mathcal{F}_{Y}^{q+1}, \kappa(y)\right) \rightarrow 0
$$

the right hand side is equal to

$$
\begin{aligned}
& \operatorname{det}\left(T-\operatorname{res}_{y} \nabla: R \Gamma\left(X_{y}, D R_{X / Y}\left(\mathcal{E}_{X}\right)\right)\right) \\
:= & \prod_{q} \operatorname{det}\left(T-\operatorname{res}_{y} \nabla: H^{q}\left(X_{y}, D R_{X / Y}\left(\mathcal{E}_{X}\right)\right)\right)^{(-1)^{q}} .
\end{aligned}
$$

We prove that $\operatorname{det}\left(T-\operatorname{res}_{y} \nabla: R \Gamma\left(X_{y}, D R_{X / Y}\left(\mathcal{E}_{X}\right)\right)\right)$ is equal to the left hand side. We take a sequence of divisors $0=D_{0}<D_{1}<\cdots<D_{\ell}=\sum_{i} \ell_{i} D_{i}<\cdots<D_{M}=$ $X_{y}$ such that $D_{\ell+1}-D_{\ell}=D_{i(\ell)}$ is irreducible. The map $[0, M) \rightarrow \amalg_{i \in I_{y}}\left[0, m_{i}\right)$ defined by $\ell \mapsto \ell_{i(\ell)} \in\left[0, m_{i(\ell)}\right)$ is a bijection. We have

$$
\begin{gathered}
\operatorname{det}\left(T-\operatorname{res}_{y} \nabla: R \Gamma\left(X_{y}, D R_{X / Y}\left(\mathcal{E}_{X}\right)\right)\right) \\
=\prod_{\ell=0}^{N-1} \operatorname{det}\left(T-\operatorname{res}_{y} \nabla: R \Gamma\left(D_{i(\ell)},\left.D R_{X / Y}\left(\mathcal{E}_{X}\left(-D_{\ell}\right)\right)\right|_{D_{i(\ell)}}\right)\right) .
\end{gathered}
$$

Since $\Phi_{i, \mathcal{E}_{X}\left(-D_{\ell}\right)}(T)=\Phi_{i, \mathcal{E}_{X}}\left(T-\ell_{i}\right)$, it is sufficient to show that

$\operatorname{det}\left(T-\operatorname{res}_{y} \nabla: R \Gamma\left(D_{i},\left.D R_{X / Y}\left(\mathcal{E}_{X}\left(-D_{\ell}\right)\right)\right|_{D_{i}}\right)\right)=N_{k_{i} / \kappa(y)}\left(\frac{\Phi_{\mathcal{E}_{X}\left(-D_{\ell}\right), i}\left(m_{i} T\right)}{m_{i}^{\text {rank } \mathcal{E}}}\right)^{c_{i}}$

for $D_{i} \subset f^{-1}(y)$. Changing the notation, we write $\mathcal{E}_{X}$ for $\mathcal{E}_{X}\left(-D_{\ell}\right)$. 
We show that $\operatorname{res}_{y} \nabla$ on $R \Gamma\left(D_{i},\left.D R_{X / Y}\left(\mathcal{E}_{X}\right)\right|_{D_{i}}\right)$ is induced by $m_{i}^{-1} \operatorname{res}_{i} \nabla$ on $\left.\mathcal{E}_{X}\right|_{D_{i}}$.

Lemma 4.4. Let $0 \rightarrow A \stackrel{a}{\rightarrow} B \stackrel{b}{\rightarrow} C \rightarrow 0$ be an exact sequence of complexes. Let $r: B \rightarrow A$ be a component-wise retraction and $s: C \rightarrow B$ be the corresponding section: $1_{B}=a r+s b$. Then the canonical map $\tilde{b}: K=\operatorname{cone}[A \rightarrow B] \rightarrow C$ admits a homotopy inverse $\tilde{s}=(s, r d s): C^{p} \rightarrow B^{p} \oplus A^{p+1}$. The composite $C \rightarrow K \rightarrow A[+1]$ is given by $r d s: C^{p} \rightarrow A^{p+1}$.

Proof. A homotopy connecting $1_{B}-\tilde{s} \circ \tilde{b}$ is given by $K^{p} \rightarrow B^{p} \stackrel{r}{\rightarrow} A^{p} \rightarrow K^{p-1}$. The rest is straightforward to check.

We apply Lemma 4.4 to the exact sequence

$$
\left.\left.\left.0 \rightarrow f^{*} \Omega_{Y}^{1}(\log E) \otimes D R_{X / Y}\left(\mathcal{E}_{X}\right)\right|_{D_{i}}[-1] \rightarrow D R_{X}\left(\mathcal{E}_{X}\right)\right|_{D_{i}} \rightarrow D R_{X / Y}\left(\mathcal{E}_{X}\right)\right|_{D_{i}} \rightarrow 0
$$

and the retraction

$$
\begin{aligned}
& m_{i}^{-1} d \log \pi \otimes i d \otimes \operatorname{res}_{i}:\left.\mathcal{E}_{X} \otimes \Omega_{X}^{q}(\log D)\right|_{D_{i}} \\
\rightarrow & \left.f^{*} \Omega_{Y}^{1}(\log E) \otimes \mathcal{E}_{X} \otimes \Omega_{X / Y}^{q-1}(\log D / \log E)\right|_{D_{i}}
\end{aligned}
$$

for a prime element $\pi$ of $\mathcal{O}_{Y, y}$. The section $s$ is induced by the canonical map

$$
\left.\left.\Omega_{X / Y}^{q}(\log D / \log E)\right|_{D_{i}} \simeq \Omega_{D_{i}}^{q}\left(\left.\log D\right|_{D_{i}}\right) \rightarrow \Omega_{X}^{q}(\log D)\right|_{D_{i}}
$$

where $\left.D\right|_{D_{i}}$ is the divisor $\bigcup_{j \neq i} D_{j} \cap D_{i}$ with simple normal crossings of $D_{i}$. By an elementary computation, the map $r d s$ in Lemma 4.4 is

$$
\begin{gathered}
m_{i}^{-1} d \log \pi \otimes \operatorname{res}_{i} \nabla \otimes i d:\left.\mathcal{E}_{X} \otimes \Omega_{X / Y}^{\bullet}(\log D / \log E)\right|_{D_{i}} \\
\left.\rightarrow f^{*} \Omega_{Y}^{1}(\log E) \otimes \mathcal{E}_{X} \otimes \Omega_{X / Y}^{\bullet}(\log D / \log E)\right|_{D_{i}} .
\end{gathered}
$$

Therefore by Lemma $4.4, \operatorname{res}_{y} \nabla$ on $R \Gamma\left(D_{i},\left.D R_{X / Y}\left(\mathcal{E}_{X}\right)\right|_{D_{i}}\right)$ is induced by $m_{i}^{-1} \operatorname{res}_{i} \nabla$ on $\left.\mathcal{E}_{X}\right|_{D_{i}}$.

To complete the proof of (2), it is sufficient to show

$$
\operatorname{det}\left(T-\operatorname{res}_{i} \nabla: R \Gamma\left(D_{i},\left.D R_{X / Y}\left(\mathcal{E}_{X}\right)\right|_{D_{i}}\right)\right)=N_{k_{i} / k} \Phi_{\mathcal{E}_{X}, i}(T)^{c_{i}} .
$$

This follows from Lemma 4.5 below applied for $X=D_{i}, \mathcal{E}=\left.\mathcal{E}_{X}\right|_{D_{i}}, f=\operatorname{res}_{i} \nabla$ and $\mathcal{F}=\left.\Omega_{X / Y}^{1}(\log D / \log E)\right|_{D_{i}} \simeq \Omega_{D_{i}}^{1}\left(\left.\log D\right|_{D_{i}}\right)$.

Lemma 4.5. Let $X$ be a proper smooth scheme of dimension $n$ over a field $k$ and let $k^{\prime}$ be the constant field of $X$. Let $\mathcal{E}$ be a torsion-free coherent $\mathcal{O}_{X}$-module and $f$ be an endomorphism of $\mathcal{E}$. Then

(1) The eigenpolynomial $\Phi(T)=\operatorname{det}(T-f: \mathcal{E}(\xi)) \in \kappa(\xi)[T]$ of $f$ at the generic point $\xi$ has coefficients in $k^{\prime}$.

Further let $\mathcal{F}$ be a locally free $\mathcal{O}_{X}$-module of rank $n$ and $c$ be the degree deg: $C H^{n}(X) \rightarrow C H^{0}\left(k^{\prime}\right)=\mathbb{Z}$ of the top Chern class $c_{n}(\mathcal{F})$. Then

(2) We have

$$
\prod_{q=0}^{n} \operatorname{det}\left(T-f \otimes i d: R \Gamma\left(X, \mathcal{E} \otimes \wedge^{q} \mathcal{F}\right)\right)^{(-1)^{q}}=N_{k^{\prime} / k} \Phi(T)^{c}
$$

in $k(T)^{\times}$. 
Proof. We may assume $k^{\prime}=k$. To show (1), it is enough to prove that the minimal polynomial $\Phi_{0}(T) \in \kappa(\xi)[T]$ of $f$ at $\xi$ divides a non-zero polynomial in $k[T]$. The minimal polynomial of $f \in \operatorname{End}_{\mathcal{O}_{X}}(\mathcal{E})$ over $k$ is in $k[T]$ and divisible by $\Phi_{0}(T)$. We show (2). By taking a direct sum decomposition, we may assume $\Phi(T)$ is a power of an irreducible polynomial $\Phi_{1}(T) \in k[T]$. Then both sides are powers of $\Phi_{1}(t)$ and it is enough to compare the degrees. By Riemann-Roch

$$
\begin{aligned}
\sum_{q=0}^{n}(-1)^{q} \chi\left(X, \mathcal{E} \otimes \wedge^{q} \mathcal{F}\right) & =\left(\left(\operatorname{ch}(\mathcal{E}) \cdot \sum_{q}(-1)^{q} \operatorname{ch}\left(\wedge^{q} \mathcal{F}\right)\right) \cdot t d \Omega_{X}^{1 *}\right)_{\operatorname{dim} 0} \\
& =\operatorname{rank} \mathcal{E} \cdot c_{n}(\mathcal{F}) .
\end{aligned}
$$

Lemma 4.5 is proved.

Thus Lemma 4.3 is proved.

Corollary. For $D_{i} \in f^{-1}(y)$, let $\nabla_{i}=\operatorname{Tr} \operatorname{res}_{i} \nabla \in k_{i}$ and $c_{i}$ be the Euler number of $D_{i}^{*} \otimes_{k_{i}} \bar{k}_{i}$. We put $m_{i}^{\nabla_{i}}=\exp \left(\nabla_{i} \log m_{i}\right) \in\left(k_{i} \otimes_{k_{0}} \mathbb{C}\right)^{\times}$where $\log m_{i} \in \mathbb{R}$. Then we have

$$
\begin{gathered}
\prod_{D_{i} \subset f^{-1}(y)} N_{k_{i} / \kappa(y)}\left(\frac{\Gamma_{D_{i}}\left(\nabla: \mathcal{E}_{X}\right)}{\Gamma_{D_{i}}\left(\nabla: \mathcal{O}_{X}(-D)\right)^{r}}\right)^{c_{i}} \\
=\prod_{q}\left(\frac{\Gamma_{y}\left(\nabla: R^{q} f_{*} D R_{X / Y}\left(\mathcal{E}_{X}\right)\right)}{\Gamma_{y}\left(\nabla: R^{q} f_{*} D R_{X / Y}\left(\mathcal{O}_{X}(-D)\right)\right)^{r}}\right)^{(-1)^{q}} \times \prod_{D_{i} \subset f^{-1}(y)} N_{k_{i} / \kappa(y)} m_{i}^{\nabla_{i} c_{i} r}
\end{gathered}
$$

in $\left(\kappa(y) \otimes_{k_{0}} \mathbb{C}\right)^{\times}$. (For the definition of $\Gamma_{D_{i}}$, see Definition 4.)

Proof. We may assume $k=\mathbb{C}$. By the formula

$$
\frac{\Gamma(s)}{\Gamma(1)}=\prod_{\ell=0}^{m-1} \frac{\Gamma((s+\ell) / m)}{\Gamma((1+\ell) / m)} \cdot m^{s},
$$

we have

$$
\Gamma\left(\frac{\Phi(T)}{(T-1)^{r}}\right)=\Gamma\left(\prod_{\ell=0}^{m-1} \frac{\Phi(m T-\ell)}{(m T-(1+\ell))^{r}}\right) \cdot m^{\Sigma(\Phi(T))}
$$

for a polynomial $\Phi(T)$ of degree $r$. For $\Phi(T)=\sum_{i=0}^{r} a_{i} T^{i}=a_{r} \prod_{i=1}^{r}\left(T-\alpha_{i}\right)$, we put $\Sigma(\Phi(T))=-a_{r-1} / a_{r}=\sum_{i=1}^{r} \alpha_{i}$.

The Corollary follows immediately from the definitions, the formula and Lemma $4.3(2)$.

(d) Global study of fibration. We continue the study of fibration $X \rightarrow Y$ to a curve. The aim in this subsection is to prove Lemma 4.6 below. We keep the assumption $\star$ in the last subsection and assume further that $Y$ and hence $X$ is proper.

Let $I_{1} \subset I$ be the index set of the components $f\left(D_{i}\right) \subset E$ and $I_{2}$ be the complement $I-I_{1}$.

Definition 8. We put $m_{\mathcal{E}_{X}}^{\nabla}=\prod_{i \in I_{1}}\left(c_{i} \cdot \exp \left(\sigma\left(\operatorname{Tr}_{k_{i} / k}\left(\operatorname{Tr}\left(\operatorname{res}_{i} \nabla\right)\right)\right) \cdot \log m_{i}\right)\right)_{\sigma} \in$ $\left(k \otimes_{k_{0}} \mathbb{C}\right)^{\times}$where $m_{i}$ is the multiplicity of $D_{i}$ in the fiber $f^{*} f\left(D_{i}\right)$ and $\log m_{i} \in \mathbb{R}$. The class of $\left[m_{\mathcal{E}_{X}}^{\nabla}\right]$ in $M P i c_{k_{0}, F}(k)$ is denoted by $m_{\mathcal{M}}^{\nabla}$. It is independent of a choice of small extension $\mathcal{E}_{X}$. The element $\prod_{i \in I_{2}} N_{k_{i} / k} \Gamma\left(\operatorname{res}_{i} \nabla: \mathcal{E}_{X}\right)^{c_{i}}$ (cf. Definition 4) in $\left(k \otimes_{k_{0}} \mathbb{C}\right)^{\times}$is denoted by $\Gamma_{2}\left(\nabla: \mathcal{E}_{X}\right)$ and the class $\left[\Gamma_{2}\left(\nabla: \mathcal{E}_{X}\right)\right]$ of $\Gamma_{2}\left(\nabla: \mathcal{E}_{X}\right)$ 
in $M P i c_{k_{0}, F}(k)$ is denoted by $\Gamma_{2}(\nabla: \mathcal{M})$. It is also independent of the choice of a small extension $\mathcal{E}_{X}$.

Lemma 4.6. We have the following equalities in $M P i c_{k_{0}, F}(k)$ :

$$
\begin{gathered}
\operatorname{per}_{c}(U, \mathcal{M})=\operatorname{per}_{c}\left(W, R f_{!} \mathcal{M}\right), \\
\frac{\Gamma(\nabla: \mathcal{M})}{\Gamma(\nabla: \mathbf{1})^{r}}=\frac{\Gamma\left(\nabla: R f_{!} \mathcal{M}\right)}{\Gamma\left(\nabla: R f_{!} \mathbf{1}\right)^{r}} \times m_{\mathcal{M}}^{\nabla} \times \Gamma_{2}(\nabla: \mathcal{M}) .
\end{gathered}
$$

If we assume that Theorem 1 holds for fibers of $f: U \rightarrow W$, we have

(3) $\left(\operatorname{det} \mathcal{M}, c_{X \bmod D}\right)=\left(\operatorname{det} R f_{!} \mathcal{M} \otimes \operatorname{det} R f_{!} 1^{\otimes-r}, c_{Y \bmod E}\right) \times m_{\mathcal{M}}^{\nabla} \times \Gamma_{2}(\nabla: \mathcal{M})$.

From Theorem 1 for $Y=\mathbb{P}_{k}^{1}$ and the Lemma, the following corollary is immediately deduced.

Corollary. If $Y=\mathbb{P}_{k}^{1}$ and if we assume that Theorem 1 holds for fibers of $f: U \rightarrow$ $W$, we have

$$
\frac{\operatorname{per}_{c}^{\Gamma}(U, \mathcal{M})}{\operatorname{per}_{c}^{\Gamma}(U, \mathbf{1})^{r}}=\left(\operatorname{det} \mathcal{M}, c_{X \bmod D}\right)
$$

(See Definition 4 for the definition of $\operatorname{per}_{c}^{\Gamma}(U, \mathcal{M})$.)

In the next subsection, we deduce Theorem 1 from the Corollary by taking a Lefschetz pencil.

Proof of Lemma 4.6. (1) Let $\left(\mathcal{E}_{X}, \nabla\right)$ be an extension of an integrable connection $(\mathcal{E}, \nabla)$ on $U$. Then we have a spectral sequence

$$
E_{2}^{p, q}=H^{p}\left(Y, D R_{Y} R^{q} f_{*} D R_{X / Y}\left(\mathcal{E}_{X}\right)\right) \Rightarrow H^{p+q}\left(X, D R_{X}\left(\mathcal{E}_{X}\right)\right) .
$$

In fact, the exact sequence of complexes

$$
0 \rightarrow D R_{X / Y}\left(\mathcal{E}_{X}\right) \otimes f^{*} \Omega_{Y}^{1}(\log E)[-1] \rightarrow D R_{X}\left(\mathcal{E}_{X}\right) \rightarrow D R_{X / Y}\left(\mathcal{E}_{X}\right) \rightarrow 0
$$

induces a quasi-isomorphism

$$
D R_{X}\left(\mathcal{E}_{X}\right) \rightarrow\left[D R_{X / Y}\left(\mathcal{E}_{X}\right) \rightarrow D R_{X / Y}\left(\mathcal{E}_{X}\right) \otimes f^{*} \Omega_{Y}^{1}(\log E)\right] .
$$

Taking the direct image, we get

$$
R f_{*} D R_{X}\left(\mathcal{E}_{X}\right) \rightarrow\left[R f_{*} D R_{X / Y}\left(\mathcal{E}_{X}\right) \rightarrow R f_{*} D R_{X / Y}\left(\mathcal{E}_{X}\right) \otimes \Omega_{Y}^{1}(\log E)\right] .
$$

The canonical filtration on $R f_{*} D R_{X / Y}\left(\mathcal{E}_{X}\right)$ induces an increasing filtration $\tau$ on $R f_{*} D R_{X}\left(\mathcal{E}_{X}\right)$ such that

$$
\begin{aligned}
G r_{q}^{\tau} R f_{*} D R_{X}\left(\mathcal{E}_{X}\right) & \simeq\left[R^{q} f_{*} D R_{X / Y}\left(\mathcal{E}_{X}\right) \rightarrow R^{q} f_{*} D R_{X / Y}\left(\mathcal{E}_{X}\right) \otimes \Omega_{Y}^{1}(\log E)\right] \\
& =D R_{Y} R^{q} f_{*} D R_{X / Y}\left(\mathcal{E}_{X}\right)[-q] .
\end{aligned}
$$

From this, we obtain the spectral sequence above by [D2], (1.4.8), taking its decalage. For a small extension $\mathcal{E}_{X}$, by Lemma 4.3 (1), the spectral sequence gives an isomorphism

$$
\operatorname{det} R \Gamma_{c}(U, D R(\mathcal{E})) \simeq \operatorname{det} R \Gamma_{c}\left(W, R f_{!} D R_{U / W}(\mathcal{E})\right) .
$$

Here we put $R f_{!} D R_{U / W}(\mathcal{E})=\left.R f_{*} D R_{X / Y}\left(\mathcal{E}_{X}\right)\right|_{W}$ for a small extension $\mathcal{E}_{X}$. It is easy to see that the isomorphism above for a small extension $\left(\mathcal{E}_{X}, \nabla\right)$ induces an isomorphism $\operatorname{det} R \Gamma_{c}(U, \mathcal{M}) \simeq \operatorname{det} R \Gamma_{c}\left(W, f_{*} \mathcal{M}\right) \in M_{k_{0}, F}(k)$ and hence (1) is proved. 
(2) It is sufficient to show an equality

$$
\frac{\Gamma_{X}\left(\nabla: \mathcal{E}_{X}\right)}{\Gamma_{X}\left(\nabla: \mathcal{O}_{X}(-D)\right)^{r}}=\frac{\Gamma_{Y}\left(\nabla: R f_{*} D R_{X / Y}\left(\mathcal{E}_{X}\right)\right)}{\Gamma_{Y}\left(\nabla: R f_{*} D R_{X / Y}\left(\mathcal{O}_{X}(-D)\right)\right)^{r}} \times m_{\mathcal{E}_{X}}^{\nabla} \times \Gamma_{2}\left(\nabla: \mathcal{E}_{X}\right)
$$

in $\left(k \otimes_{k_{0}} \mathbb{C}\right)^{\times}$. It is an immediate consequence of the Corollary of Lemma 4.5.

(3) By Lemma 2.1 for partial trivializations res and $m_{i}^{-1} \operatorname{res}_{i}$ of $\Omega_{X}^{1}(\log D)$ and by Lemma 2.2 applied to the exact sequence

$$
0 \rightarrow f^{*} \Omega_{Y}^{1}(\log E) \rightarrow \Omega_{X}^{1}(\log D) \rightarrow \Omega_{X / Y}^{1}(\log D / \log E) \rightarrow 0,
$$

we have

$$
c_{X \bmod D}=f^{*} c_{Y \bmod E} \cup c_{X / Y \bmod D / E}-\sum_{i \in I_{1}} m_{i} i_{i *} c_{D_{i}} .
$$

Since $\left(\operatorname{det} \mathcal{M}, \sum_{i \in I_{1}} m_{i} i_{i *} c_{D_{i}}\right)=m_{\mathcal{M}}^{\nabla}$ by the definition of the tame symbol, we have an equality

$$
\left(\operatorname{det} \mathcal{M}, c_{X \bmod D}\right)=\left(\operatorname{det} \mathcal{M}, f^{*} c_{Y \bmod E} \cup c_{X / Y \bmod D / E}\right) \times m_{\mathcal{M}}^{\nabla} .
$$

Therefore it is sufficient to prove

$$
\begin{aligned}
& \left(\operatorname{det} R f_{!} \mathcal{M} \otimes \operatorname{det} R f_{!} \mathbf{1}^{\otimes-r}, c_{Y \bmod E}\right) \times \Gamma_{2}(\nabla: \mathcal{M}) \\
= & \left(\operatorname{det} \mathcal{M}, f^{*} c_{Y \bmod E} \cup c_{X / Y \bmod D / E}\right) .
\end{aligned}
$$

Lemma 4.7. There is a dense open subset $W^{\prime} \subset W$ such that for $y \in W^{\prime}$ the restriction $\left.\mathcal{E}_{X}\right|_{X_{y}}=\mathcal{E}_{X} \otimes \mathcal{O}_{X} \mathcal{O}_{X_{y}}$ is also reflexive.

We finish the proof of Lemma 4.6 admitting Lemma 4.7. By Lemma 4.7 and the Corollary of Proposition 1 in Section 2, we may write $c_{Y \bmod E}=\sum_{y \in W^{\prime}} n_{y}[y]$. Then by proper base change and by the definition of $\Gamma_{2}(\nabla: \mathcal{M})$, the left hand side is

$$
\prod_{y \in W^{\prime}} N_{y / k}\left(\frac{\operatorname{per}_{c}^{\Gamma}\left(U_{y},\left.\mathcal{M}\right|_{U_{y}}\right)}{\left(\operatorname{per}_{c}^{\Gamma}\left(U_{y}, \mathbf{1}_{U_{y}}\right)\right)^{r}}\right)^{n_{y}}
$$

In fact, by $c_{i}=\sum_{y} n_{y} \operatorname{deg} y \cdot c_{i}^{\prime}$ where $c_{i}^{\prime}$ is the Euler number of a geometric fiber $\left.D_{i}^{*}\right|_{W} \rightarrow W$ for $i \in I_{2}$, we have $\Gamma_{2}\left(\nabla: \mathcal{E}_{X}\right)=\prod_{y \in W^{\prime}} N_{y / k} \Gamma\left(\nabla:\left.\mathcal{E}_{X}\right|_{X_{y}}\right)^{n_{y}}$ for a small extension $\mathcal{E}_{X}$. On the other hand,

$$
\left(\operatorname{det} \mathcal{M}, f^{*} c_{Y \bmod E} \cup c_{X / Y \bmod D / E}\right)=\prod_{y \in W^{\prime}} N_{y / k}\left(\left.\operatorname{det} \mathcal{M}\right|_{U_{y}}, c_{X_{y} \bmod D_{y}}\right)^{n_{y}} .
$$

In fact for the Gysin map $i_{y *}: C H^{n-1}\left(X_{y} \bmod D_{y}\right) \rightarrow C H^{n}(X \bmod D)$, we have $f^{*} c_{Y \bmod E} \cup c_{X / Y \bmod D / E}=\sum_{y \in W^{\prime}} n_{y} i_{y *}\left(c_{X_{y} \bmod D_{y}}\right)$ and $\left(\operatorname{det} \mathcal{M}, i_{y *} c\right)=$ $\left(\left.\operatorname{det} \mathcal{M}\right|_{U_{y}}, c\right)$.

Therefore to complete the proof of Lemma 4.6, it is enough to show Lemma 4.7.

Proof of Lemma 4.7. Let ${ }^{*}$ denote the dual. Let $W^{\prime}$ be a dense open subset such that for any closed point $y \in W^{\prime}$ the fiber $X_{y}$ does not contain any component of the associated cycles $A s s\left(\mathcal{E}_{x} t_{\mathcal{O}_{X}}^{1}\left(\mathcal{E}_{X}, \mathcal{O}_{X}\right)\right)$ and $A s s\left(\mathcal{E} x t_{\mathcal{O}_{X}}^{1}\left(\mathcal{E}_{X}^{*}, \mathcal{O}_{X}\right)\right)$. We show for $y \in W^{\prime}$ the canonical morphisms $\left.\mathcal{E}_{X}^{*}\right|_{X_{y}} \rightarrow\left(\left.\mathcal{E}_{X}\right|_{X_{y}}\right)^{*}$ and $\left.\mathcal{E}_{X}^{* *}\right|_{X_{y}} \rightarrow\left(\left.\mathcal{E}_{X}^{*}\right|_{X_{y}}\right)^{*}$ are isomorphisms. This proves the Lemma because the canonical map $\left.\mathcal{E}_{X}\right|_{X_{y}} \rightarrow$ 
$\left(\left.\mathcal{E}_{X}\right|_{X_{y}}\right)^{* *}$ is also an isomorphism. We show the assertion for $\mathcal{E}_{X}$ and the same argument works for $\mathcal{E}_{X}^{*}$. For a closed point $y \in W^{\prime}$, by the exact sequence

$$
\left.0 \rightarrow \mathcal{E}_{X}^{*}\right|_{X_{y}} \rightarrow\left(\left.\mathcal{E}_{X}\right|_{X_{y}}\right)^{*} \rightarrow \mathcal{E} x t_{\mathcal{O}_{X}}^{1}\left(\mathcal{E}_{X}, \mathcal{O}\left(-X_{y}\right)\right),
$$

and by the assumption that $\mathcal{E} t_{\mathcal{O}_{X}}^{1}\left(\mathcal{E}_{X}, \mathcal{O}\left(-X_{y}\right)\right)$ has no submodule supported on the fiber $X_{y}$, the canonical morphism $\left.\mathcal{E}_{X}^{*}\right|_{X_{y}} \rightarrow\left(\left.\mathcal{E}_{X}\right|_{X_{y}}\right)^{*}$ is an isomorphism. Thus Lemma 4.7 is proved.

(e) End of proof. We prove the general case by induction on $\operatorname{dim} X$. If $\operatorname{dim} X=0$, this is clear. Taking a Lefschetz pencil, we complete the proof following Step 2 and Step 3 in the proof of [S1], Theorem 1. By Lemma 3 loc.cit., there exists a Lefschetz pencil $\left(H_{y}\right)_{y \in \mathbb{P}_{k}^{1}}$ of $X$ satisfying the following properties (1)-(4).

(1) The axis of the pencil meets $D_{J}=\bigcap_{i \in J} D_{i}$ transversally for all $J \subset I$.

(2) There exists a dense open subscheme $W \subset Y=\mathbb{P}_{k}^{1}$ such that for all $y \in W$, the hyperplane $H_{y}$ meets $D_{J}$ transversally for all $J \subset I$.

(3) For all $y \in Y$ and all $J \subset I$, the hyperplane $H_{y}$ meets $D_{J}$ transversally except at isolated ordinary quadratic singularities.

For $J \subset I$, let $S_{J}=\left\{x \in D_{J} ; x\right.$ is a singular point of $H_{y} \subset D_{J}$ for some $\left.y \in Y\right\}$. Then

(4) $S_{J}$ and $S_{J^{\prime}}$ are disjoint for all $J \neq J^{\prime}, \subset I$.

We define a blowing-up $X_{3}$ of $X$, a morphism $f_{3}: X_{3} \rightarrow Y=\mathbb{P}_{k}^{1}$ and a divisor $D_{3}$ with simple normal crossings satisfying the condition $\star$ of (c) as follows. Let $X_{1}$ be the blowing-up of $X$ with the center the intersection $Z$ of $X$ with the axis of the pencil and $f_{1}: X_{1} \rightarrow Y=\mathbb{P}_{k}^{1}$ be the map defined by the pencil. Let $S \subset X_{1}$ be the disjoint union $S=\amalg_{J} S_{J}$. Let $X_{2}$ be the blowing up of $X_{1}$ at $S$. For $x \in S_{J}$ and $J \neq \emptyset$, let $B_{x}$ be the intersection of the exceptional divisor $E_{x}$ and the proper transform of $D_{J}$ in $X_{2}$. Let $X_{3}$ be the blowing-up of $X_{2}$ at all $B_{x}$ for $x \in S-S_{\emptyset}$. We put $E=f_{1}(S), W=Y-f_{1}(S)$ and let $D_{3}$ be the divisor $\varphi_{3}^{*} D \cup f_{3}^{*} E$ where $f_{3}: X_{3} \rightarrow Y$ and $\varphi_{3}: X_{3} \rightarrow X$. Then it is easy to check that $D_{3}$ has simple normal crossings and $f_{3}: X_{3} \rightarrow Y$ satisfies the condition $\star$.

We have proved the Theorem for $\left(X_{3}, D_{3}\right)$ by the Corollary of Lemma 4.6 by induction. Therefore the following Lemma completes the proof of Theorem 1.

Lemma 4.8. Let $X, D$ and $\mathcal{M}$ be as in the statement of Theorem 1. Assume Theorem 1 holds for lower dimensions. Let $Z$ be a regular closed subscheme of $X$ satisfying one of the following conditions (1)-(3) and let $\varphi: X^{\prime} \rightarrow X$ be the blowingup at $Z$. Then Theorem 1 for $\mathcal{M}$ on $U$ is equivalent to that for $\mathcal{M}^{\prime}=\varphi^{*} \mathcal{M}$ on $U^{\prime}=\varphi^{*}(U)$.

(1) $Z$ is a regular closed subscheme of codimension 2 meeting $D_{J}$ transversally for all $J \subset I$.

(2) $Z=\{x\}$ for a closed point $x$ of $X$.

(3) $Z=D_{J}$ for some $J \subset I$.

Proof. Let $D^{\prime}$ be the divisor $\varphi^{*} D=X^{\prime}-U^{\prime}$ with simple normal crossing.

(1) Let $D_{Z}=D \cap Z$ and $Z^{*}=Z-D_{Z}$. It is easy to see that

$$
\operatorname{per}_{c}^{\Gamma}\left(U^{\prime}, \varphi^{*} \mathcal{M}\right)=\operatorname{per}_{c}^{\Gamma}(U, \mathcal{M}) \times \operatorname{per}_{c}^{\Gamma}\left(Z^{*},\left.\mathcal{M}\right|_{Z^{*}}(-1)\right) .
$$


We show $\left(\operatorname{det} \varphi^{*} \mathcal{M}, c_{X^{\prime} \bmod D^{\prime}}\right)_{X^{\prime}}=\left(\operatorname{det} \mathcal{M}, c_{X \bmod D}\right)_{X} \times\left(\left.\operatorname{det} \mathcal{M}\right|_{Z^{*}}, c_{Z \bmod Z^{*}}\right)_{Z}$. We apply Corollary 2 of Lemma 2.3 to the exact sequence

$$
0 \rightarrow \varphi^{*} \Omega_{X}^{1}(\log D) \rightarrow \Omega_{X^{\prime}}^{1}\left(\log D^{\prime}\right) \rightarrow \Omega_{E / Z}^{1} \rightarrow 0 .
$$

By the exact sequence

$$
\begin{aligned}
& 0 \rightarrow N_{E / X}\left(=\mathcal{O}_{E}(1)\right) \rightarrow \text { Image }\left(\left.\left.\varphi^{*} \Omega_{X}^{1}(\log D)\right|_{E} \rightarrow \Omega_{X^{\prime}}^{1}\left(\log D^{\prime}\right)\right|_{E}\right) \\
& \rightarrow \varphi^{*} \Omega_{Z}^{1}\left(\log D_{Z}\right) \rightarrow 0,
\end{aligned}
$$

we obtain

$$
c_{X^{\prime} \bmod D^{\prime}}=\varphi^{*} c_{X \bmod D}+i_{E_{*}}\left(\varphi^{*} c_{Z \bmod D_{Z}} \cup c_{1}\left(\mathcal{O}_{E}(1)\right)\right)
$$

in $C H^{n}\left(X^{\prime} \bmod D^{\prime}\right)$. By writing an element of the relative Chow group by 0-cycles (the Corollary of Proposition 1 in Section 2), we have equalities $\left(\operatorname{det} \varphi^{*} \mathcal{M}, \varphi^{*} c\right)_{X^{\prime}}=$ $(\operatorname{det} \mathcal{M}, c)_{X}$ for $c \in C H^{n}(X \bmod D),\left(\operatorname{det} \varphi^{*} \mathcal{M}, i_{E_{*}} c\right)=\left(\left.\operatorname{det} \mathcal{M}\right|_{E \cap U^{\prime}}, c\right)$ for $c \in$ $C H^{n-1}\left(E \bmod D^{\prime} \cap E\right)$ and $\left(\operatorname{det} \varphi^{*} \mathcal{M}, i_{E_{*}}\left(\varphi^{*} c \cup c_{1}\left(\mathcal{O}_{E}(1)\right)\right)\right)=\left(\left.\operatorname{det} \mathcal{M}\right|_{Z^{*}}, c\right)$ for $c \in C H^{n-2}\left(Z \bmod D_{Z}\right)$. By the equalities above, we obtain the required equality. Under Theorem 1 for $Z,(1)$ is proved.

(2) for $x \in U$. It is easy to see that

$$
\operatorname{per}_{c}^{\Gamma}\left(U^{\prime}, \varphi^{*} \mathcal{M}\right)=\operatorname{per}_{c}^{\Gamma}(U, \mathcal{M}) \times \prod_{q=1}^{n-1} N_{x / k}(\operatorname{det}(\mathcal{M}(-q))(x)) .
$$

We show $\left(\operatorname{det} \varphi^{*} \mathcal{M}, c_{X^{\prime} \bmod D^{\prime}}\right)_{X^{\prime}}=\left(\operatorname{det} \mathcal{M}, c_{X \bmod D}\right)_{X} \times N_{x / k} \operatorname{det} \mathcal{M}(x)^{n-1}$. By Corollary 1 of Lemma 2.3 applied to the exact sequence

$$
0 \rightarrow \varphi^{*} \Omega_{X}^{1}(\log D) \rightarrow \Omega_{X^{\prime}}^{1}\left(\log D^{\prime}\right) \rightarrow \Omega_{E}^{1} \rightarrow 0
$$

and by an elementary computation, we obtain

$$
c_{X^{\prime} \bmod D^{\prime}}=\varphi^{*} c_{X \bmod D}+i_{E_{*}}\left((n-1)\left[x^{\prime}\right]\right)
$$

in $C H^{n}\left(X^{\prime} \bmod D^{\prime}\right)$ for a $\kappa(x)$-rational point $x^{\prime} \in E$. Similarly as in (1), we obtain the equality.

(2) for $x \in D$ and (3). In these cases, $\operatorname{per}_{c}^{\Gamma}\left(U^{\prime}, \varphi^{*} \mathcal{M}\right)=\operatorname{per}_{c}^{\Gamma}(U, \mathcal{M})$. In fact, except for the case (2) where Card $I_{x}=1$, the Euler numbers $c_{i}$ of $D_{i}^{*}$ do not change and that for the exceptional divisor $E^{*}$ is 0 . In the exceptional case, the Euler number of $c_{i_{0}}$ for $x \in D_{i_{0}}$ decreases by 1 , that for $E^{*}$ is 1 and the characteristic polynomials $\Phi_{\mathcal{E}_{X}}$ for $D_{i_{0}}$ and for $E$ are the same. We show $\left(\operatorname{det} \varphi^{*} \mathcal{M}, c_{X^{\prime} \bmod D^{\prime}}\right)_{X^{\prime}}=\left(\operatorname{det} \mathcal{M}, c_{X \bmod D}\right)_{X}$. Let $D_{1}$ be a regular family of subschemes $D \cup\{Z\}, \rho_{1}$ be the partial trivialization of $\Omega_{X}^{1}(\log D)$ defined by $\operatorname{res}_{i}$ for $D_{i}$ and $\sum_{D_{i} \supset Z}$ res $_{i}$ for $Z$ and let $\rho_{1}^{\prime}$ be the partial trivialization of $\varphi^{*} \Omega_{X}^{1}(\log D)$ on $D^{\prime}$ defined by pulling-back $\rho_{1}$. We consider the canonical maps

$$
H^{n}\left(X, \mathcal{K}_{n}(X \bmod D)\right) \leftarrow H^{n}\left(X, \mathcal{K}_{n}\left(X \bmod D_{1}\right)\right) \rightarrow H^{n}\left(X^{\prime}, \mathcal{K}_{n}\left(X^{\prime} \bmod D^{\prime}\right)\right) .
$$

The first one is an isomorphism since $H^{n-1}\left(Z, \mathcal{K}_{n}(Z)\right)=0$ and $H^{n}\left(Z, \mathcal{K}_{n}(Z)\right)=0$ by $\operatorname{dim} Z \leq n-2$. The images of the relative top Chern class $c_{n}\left(\Omega_{X}^{1}(\log D), \rho_{1}\right)$ are $c_{n}\left(\Omega_{X}^{1}(\log D)\right.$, res $)$ and $c_{n}\left(\varphi^{*} \Omega_{X}^{1}(\log D), \varphi^{*} \rho_{1}\right)$ respectively. Further by Lemma 2.4 applied to $\varphi^{*} \Omega_{X}^{1}(\log D) \rightarrow \Omega_{X^{\prime}}^{1}(\log D)$, we have $c_{n}\left(\varphi^{*} \Omega_{X}^{1}(\log D), \varphi^{*} \rho_{1}\right)=$ $c_{n}\left(\Omega_{X^{\prime}}^{1}(\log D)\right.$, res $)$. Thus we obtain an equality

$$
\left(\operatorname{det} \varphi^{*} \mathcal{M}, c_{X^{\prime} \bmod D^{\prime}}\right)_{X^{\prime}}=\left(\operatorname{det} \mathcal{M}, c_{X \bmod D}\right)_{X}
$$

by the Corollary of Proposition 1 and Lemma 4.8 is proved. 
Now the proof of Theorem 1 is completed.

(f) A variant. We give a variant of Theorem 1 for the category defined below, which will be used in the application given in the next section. Let $k_{0}$ be a subfield of $\mathbb{C}$ and $k$ and $F$ be finite extensions of $k_{0}$. Let $U$ be a smooth scheme over $k$. For an integrable connection $\nabla: \mathcal{E} \rightarrow \mathcal{E} \otimes \Omega_{U}^{1}$, we call a multiplication by $F$ on $(\mathcal{E}, \nabla)$ a $k_{0}$-homomorphism $F \rightarrow E n d_{\mathcal{O}_{X}}^{\nabla}(\mathcal{E})=\operatorname{Ker}\left(\nabla\right.$ on $\left.\operatorname{End}_{\mathcal{O}_{X}}(\mathcal{E})\right)$.

Definition 9. We define a category $M_{k_{0}, F}^{\prime}(U)$ whose objects are triples $\mathcal{M}=$ $((\mathcal{E}, \nabla), V, \rho)$ consisting of

(1) An integrable connection $(\mathcal{E}, \nabla)$ on $U$ regular along boundary with a multiplication by $F$.

(2) A local system $V$ of $F$-vector spaces on $U^{a n}$.

(3) A morphism $\rho: V \rightarrow \mathcal{E}^{a n}$ on $U^{a n}$ inducing an isomorphism $V \otimes_{k_{0}} \mathbb{C} \rightarrow$ Ker $\nabla^{a n}$ of local systems of $F \otimes_{k_{0}} \mathbb{C}$-modules.

For this category, we have analogues of Theorems 1 and 2 . We define briefly both sides of the isomorphisms there. Note that an integrable connection $(\mathcal{E}, \nabla)$ with a multiplication by $F$ is naturally identified with an integrable connection denoted $\left(\mathcal{E}^{(F)}, \nabla^{(F)}\right)$ on $U \otimes_{k_{0}} F$. Let $X$ be a proper smooth scheme over $k$ including $U$ as the complement of a divisor $D$ with simple normal crossings. For an integrable connection $(\mathcal{E}, \nabla)$ on $U$ with a multiplication by $F$, it is regular along $D$ if and only if $\left(\mathcal{E}^{(F)}, \nabla^{(F)}\right)$ is regular along $D \otimes_{k_{0}} F$. Hence if it is regular, there exists an extension $\left(\mathcal{E}_{X}, \nabla\right)$ to $X$ with an extended multiplication $F \rightarrow \operatorname{End}_{\mathcal{O}_{X}}^{\nabla}\left(\mathcal{E}_{X}\right)$. The rank of an object $\mathcal{M}=((\mathcal{E}, \nabla), V, \rho)$ of $M_{k_{0}, F}^{\prime}(U)$ is the rank of $\mathcal{E}^{(F)}$ as an $\mathcal{O}_{U \otimes F}$-module which is the same as the rank of $V$ as a local system of $F$-vector spaces. The determinant $\operatorname{det}_{F}(\mathcal{E}, \nabla)$ over $F$ of an integrable connection $(\mathcal{E}, \nabla)$ with a multiplication by $F$ is defined as that corresponding to $\operatorname{det}_{\mathcal{O}_{U \otimes F}} \mathcal{E}^{(F)}$. The class group $M P i c_{k_{0}, F}^{\prime}(U)$ of the rank 1 objects of $M_{k_{0}, F}^{\prime}(U)$ is defined similarly as in Section 1. For a finite extension $k$ of $k_{0}$, there is a canonical isomorphism $M P i c_{k_{0}, F}^{\prime}(k)=\left(k \otimes_{k_{0}} F\right)^{\times} \backslash\left(k \otimes_{k_{0}} F \otimes_{k_{0}} \mathbb{C}\right)^{\times} /\left(F^{\times}\right)^{\operatorname{Hom}_{k_{0}}(k, \mathbb{C})}$.

For an object $\mathcal{M}=((\mathcal{E}, \nabla), V, \rho)$ of $M_{k_{0}, F}^{\prime}(U)$, the determinant of cohomology $\operatorname{det}_{F} R \Gamma_{c}(U, \mathcal{M})$ is a rank 1 object in $M_{k_{0}, F}^{\prime}(k)$. It is the alternating tensor product of the determinant over $F$ of the compact support cohomology

$$
H_{c}^{q}(U, \mathcal{M})=\left(H^{q}\left(X, D R\left(\mathcal{E}_{X}\right)\right), H_{c}^{q}\left(U^{a n}, V\right), H_{c}^{q}(\rho)\right) \in M_{k_{0}, F}^{\prime}(k)
$$

where $\mathcal{E}_{X}$ is a small extension of $\mathcal{E}$ with a multiplication by $F$. The period (with compact support) $\operatorname{per}_{c, F}(\mathcal{M}) \in M P i c_{k_{0}, F}^{\prime}(k)$ is the class of $\operatorname{det}_{F} R \Gamma_{c}(U, \mathcal{M})$. We also define a variant $\operatorname{per}_{c, F}^{\Gamma}(\mathcal{M}) \in M P i c_{k_{0}, F}^{\prime}(k)$ as follows. Take a small extension $\left(\mathcal{E}_{X}, \nabla\right)$ with an extended multiplication by $F$. We define $\Gamma_{F}\left(\nabla: \mathcal{E}_{X}\right) \in(k \otimes F \otimes \mathbb{C})^{\times}$ as follows. By $k \otimes F=\prod_{j} K_{j}$ for fields $K_{j}$, the fiber product $X \otimes_{\mathbb{Q}} F$ is the disjoint union $\amalg X_{j}$. Let $\mathcal{E}_{X_{j}}$ be the restriction on $X_{j}$ of the corresponding connection $\mathcal{E}_{X}^{(F)}$. Then $\Phi_{\mathcal{E}_{X_{j}}}(T) \in K_{j}(T)^{\times}$and $\Gamma\left(\nabla: \mathcal{E}_{X_{j}}\right)=\Gamma\left(\Phi_{\mathcal{E}_{X_{j}}}(T)\right) \in\left(K_{j} \otimes_{\mathbb{Q}} \mathbb{C}\right)^{\times}$are defined in Section 1. We put $\Gamma_{F}\left(\nabla: \mathcal{E}_{X}\right)=\left(\Gamma\left(\nabla: \mathcal{E}_{X_{j}}\right)\right)_{j} \in \prod_{j}\left(K_{j} \otimes_{\mathbb{Q}} \mathbb{C}\right)^{\times}=(k \otimes F \otimes \mathbb{C})^{\times}$. Its class $\Gamma_{F}(\nabla: \mathcal{M})$ in $M P i c_{k_{0}, F}^{\prime}(k)=\left(k \otimes_{k_{0}} F\right)^{\times} \backslash\left(k \otimes_{k_{0}} F \otimes_{k_{0}} \mathbb{C}\right)^{\times} /\left(F^{\times}\right)^{H_{o m} m_{k_{0}}}(k, \mathbb{C})$ is independent of the choice of a small extension and is well defined. Similarly as in Section 1, we define $\operatorname{per}_{c, F}^{\Gamma}(U, \mathcal{M})$ to be the product $\operatorname{per}_{c, F}(U, \mathcal{M}) \times \Gamma_{F}(\nabla: \mathcal{M})^{-1}$. 
We also define a pairing

$$
M P i c_{k_{0}, F, X}^{\prime}(U) \times C H^{n}(X \bmod D) \rightarrow M P i c_{k_{0}, F}^{\prime}(k) .
$$

As is shown in Section 3, the essential point is the definition of the tame symbol $M P i c_{k_{0}, F, X}^{\prime}(U) \times A_{x} \rightarrow M P i c_{k_{0}, F}^{\prime}(x)$ for a closed point $x \in X$. Let $\mathcal{M}=$ $((\mathcal{E}, \nabla), V, \rho) \in M_{k_{0}, F}^{\prime}(U)$ be a triple of rank 1 and $f \in A_{x}$. Then in the same way as in Section 3 , by taking an extension $\mathcal{E}_{X}$ which is an invertible $\mathcal{O}_{X} \otimes_{k_{0}} F$ module, a triple $(\mathcal{M}, f)_{x} \in M_{k_{0}, F}^{\prime}(x)$ is defined. This construction induces the pairing $M P i c_{k_{0}, F, X}^{\prime}(U) \times C H^{n}(X \bmod D) \rightarrow M P i c_{k_{0}, F}^{\prime}(k)$.

By the same argument as above, we have

Theorem $\mathbf{1}^{\prime}$. Assume $X$ is projective. Then for an object $\mathcal{M}$ of $M_{k_{0}, F}^{\prime}(U)$, we have an equality

$$
\operatorname{per}_{c, F}^{\Gamma}(U, \mathcal{M})=\operatorname{per}_{c, F}^{\Gamma}(U, \mathbf{1})^{\operatorname{rank} \mathcal{M}} \cdot\left(\operatorname{det}_{F} \mathcal{M}, c_{X \bmod D}\right)
$$

in $M P i c_{k_{0}, F}^{\prime}(k)$.

\section{5. $\ell$-ADiC Sheaves. Motives}

(a) Tame symbol for abelian fundamental group. In this section, we first define a pairing

$$
C H^{n}(X \bmod D) \times \pi_{1}(k)^{\mathrm{ab}} \rightarrow \pi_{1}(U)^{\mathrm{ab}, \mathrm{tame}}
$$

compatible with the pairings

$$
C H^{n}(X) \times \pi_{1}(k)^{\mathrm{ab}} \rightarrow \pi_{1}(X)^{\mathrm{ab}}
$$

defined in [S2], Proposition 1. Let $k$ be a field, $X$ be a proper regular scheme over $k$ and $U$ be an open subscheme whose complement $D$ is a divisor with simple normal crossings. As in [S2], let $\pi_{1}(U)^{\text {ab,tame }}$ be the quotient of $\pi_{1}(U)^{\text {ab }}$ classifying the abelian coverings of $U$ tamely ramified along $D$. It is the Pontrjagin dual of $H^{1}(U, \mathbb{Q} / \mathbb{Z})^{\text {tame }}=H^{1}(U, \mathbb{Q} / \mathbb{Z}[1 / p]) \oplus H^{1}\left(X, \mathbb{Q}_{p} / \mathbb{Z}_{p}\right)$ for $p=\operatorname{char} k$.

Proposition 3. Let $X$ and $U$ be defined over a field $k$ as above. Then there is a pairing

$$
C H^{n}(X \bmod D) \times \pi_{1}(k)^{\mathrm{ab}} \rightarrow \pi_{1}(U)^{\mathrm{ab}, \mathrm{tame}}
$$

characterized by the following property. For a closed point $x \in U$, the pairing with the class $[x]$ is given by the composition

$$
\pi_{1}(k)^{\mathrm{ab}} \stackrel{[\kappa(x): k]_{i} \cdot t r_{x / k}}{\longrightarrow} \pi_{1}(x)^{\mathrm{ab}} \stackrel{i_{x *}}{\longrightarrow} \pi_{1}(U)^{\mathrm{ab}, \text { tame }}
$$

of the inseparable degree times the transfer $t r_{x / k}$ and the map induced by the inclusion $i_{x}$.

Proof. By the Corollary of Proposition 1, it is unique. By taking the dual it is sufficient to define a pairing

$$
H^{1}(U, \mathbb{Q} / \mathbb{Z})^{\text {tame }} \times C H^{n}(X \bmod D) \rightarrow H^{1}(k, \mathbb{Q} / \mathbb{Z})
$$

such that, for a closed point $x \in X_{0}$, the pairing with the class $[x]$ is the composition $H^{1}(U, \mathbb{Q} / \mathbb{Z})^{\text {tame }} \stackrel{i_{x}^{*}}{\rightarrow} H^{1}(x, \mathbb{Q} / \mathbb{Z}) \stackrel{[\kappa(x): k]_{i} \times \operatorname{Tr}_{x / k}}{\longrightarrow} H^{1}(k, \mathbb{Q} / \mathbb{Z})$. For the $p=\operatorname{char} k-$ part, this is Proposition 1 of [S2]. There $X$ is assumed to be smooth but it is enough to only assume $X$ is regular. Hence it is enough to define a pairing

$$
H^{1}(U, \mathbb{Z} / m) \times C H^{n}(X \bmod D) \rightarrow H^{1}(k, \mathbb{Z} / m)
$$


for an integer $m \in \mathbb{Z}$ invertible in $k$. Let $\kappa: \Gamma\left(U, \mathbb{G}_{m}\right) \rightarrow H^{1}\left(U, \mu_{m}\right)$ be the map induced by the Kummer sequence.

First we assume $X$ is a proper regular integral curve. Let $\mathbb{G}_{m}(X \bmod D)$ be the complex $\left[\mathbb{G}_{m, X} \rightarrow \mathbb{G}_{m, D}\right]$ on $X^{e t}$. The Kummer sequence induces a map $H^{1}\left(X, \mathbb{G}_{m}(X \bmod D)\right) \rightarrow H_{c}^{2}\left(U, \mu_{m}\right)$. The pairing $\operatorname{Tr}_{U / k} \circ \cup: H^{1}(U, \mathbb{Z} / m) \times$ $H_{c}^{2}\left(U, \mu_{m}\right) \rightarrow H^{1}(k, \mathbb{Z} / m)$ induces a pairing

$$
H^{1}(U, \mathbb{Z} / m) \times C H^{1}(X \bmod D) \rightarrow H^{1}(k, \mathbb{Z} / m)
$$

By the canonical isomorphism $C H^{1}(X \bmod D) \rightarrow H^{1}\left(X, \mathbb{G}_{m}(X \bmod D)\right)$, the required pairing is given.

For general $X$, similarly as in Section 3, we define a local pairing

$$
H^{1}(U, \mathbb{Z} / m) \times A_{x} \rightarrow H^{1}(x, \mathbb{Z} / m)
$$

for a closed point $x \in X_{0}$ using the tame symbol and check the reciprocity for $y \in X_{1}$. For a while, we drop the assumption that $X$ is proper and we define the tame symbol in this context. Let $J \subset I$ be a subset of the index set of the irreducible components of $D$ and let $T^{*}=T-\bigcup_{i \in I-J} D_{i} \subset T=\bigcap_{i \in J} D_{i}$ and $A_{J}=\left(\prod_{i \in J}\right)_{\mathbb{Z}} \Gamma\left(X-D_{i}, \mathbb{G}_{m}\right)$ be the fiber product as in Section 3. We define a pairing

$$
H^{1}(U, \mathbb{Z} / m) \times A_{J} \rightarrow H^{1}\left(T^{*}, \mathbb{Z} / m\right)
$$

Replacing $X$ by $X-\bigcup_{i \in I-J} D_{i}$, we may assume $T=T^{*}$ and $I=J$ and we put $A=A_{J}$. It is enough to define the pairing Zariski locally on $X$ and patch them afterward. Hence we may assume that the ideal sheaf $\mathcal{O}\left(-D_{i}\right)$ is a trivial invertible $\mathcal{O}_{X}$-module and $\operatorname{ord}_{i}: A_{i} \rightarrow \mathbb{Z}$ is surjective for $i \in I$. We show that the heuristic argument in the Remark in Section 3 actually works. For each $i \in I$, the Kummer sequence induces a map $A_{i} \rightarrow H^{1}\left(X-D_{i}, \mathbb{Z} / m(1)\right) \rightarrow H_{D_{i}}^{2}\left(X, j_{i} \mathbb{Z} / m(1)\right)$ where $j_{i}: X-D_{i} \rightarrow X$ is the open immersion. Let $\tilde{A}$ be the tenser product $\tilde{A}=\bigotimes_{i \in I} A_{i}$ and $r=\operatorname{Card} I$. By taking the cup-product, we obtain $\tilde{A} \rightarrow H_{T}^{2 r}(X, j ! \mathbb{Z} / m(r))$. The cup-product

$$
H^{1}(U, \mathbb{Z} / m) \times H_{T}^{2 r}(X, j ! \mathbb{Z} / m(r)) \rightarrow H_{T}^{2 r+1}(X, \mathbb{Z} / m(r)) \simeq H^{1}(T, \mathbb{Z} / m)
$$

induces a pairing $():, H^{1}(U, \mathbb{Z} / m) \times \tilde{A} \rightarrow H^{1}(T, \mathbb{Z} / m)$. Let $\tilde{A}^{2}$ be the subgroup of $\tilde{A}$ generated by $\bigotimes_{i} a_{i}$ such that $\operatorname{ord}_{i} a_{i}=0$ for at least two $i \in I$. Since $\operatorname{ord}_{i}: A_{i} \rightarrow \mathbb{Z}$ are surjective, the fiber product $A$ is isomorphic to $\tilde{A} / \tilde{A}^{2}$ by $\bigotimes a_{i} \mapsto$ $\left(a_{i}^{\prod_{j \neq i} \operatorname{ord}_{j} a_{j}}\right)_{i}$. We show that the pairing $($,$) annihilates \tilde{A}^{2}$. We may assume Card $I=2$ and $I=\{1,2\}$. The pairing factors as

$$
\begin{aligned}
H^{1}(U, \mathbb{Z} / m) \times A_{1} \times A_{2} \stackrel{(,)_{1} \times \text { res }}{\longrightarrow} H^{1}\left(D_{1}-T, \mathbb{Z} / m\right) \times \Gamma\left(D_{1}-T, \mathbb{G}_{m}\right) \\
\stackrel{(,)_{2}}{\longrightarrow} H^{1}(T, \mathbb{Z} / m) .
\end{aligned}
$$

Further, in the first pairing $(,)_{1}$, the restriction to $\Gamma\left(X, \mathbb{G}_{m}\right) \subset A_{1}$ is

$$
\begin{aligned}
H^{1}(U, \mathbb{Z} / m) \times \Gamma\left(X, \mathbb{G}_{m}\right) \stackrel{\partial \times(\kappa \circ \text { res })}{\longrightarrow} H^{0}\left(D_{1}, \mathbb{Z} / m(-1)\right) \times H^{1}\left(D_{1}, \mathbb{Z} / m(1)\right) \\
\stackrel{\cup}{\longrightarrow} H^{1}\left(D_{1}, \mathbb{Z} / m\right) .
\end{aligned}
$$

Here the first arrow is the product of the boundary map and the restriction followed by $\kappa: \Gamma\left(D_{1}, \mathbb{G}_{m}\right) \rightarrow H^{1}\left(D_{1}, \mathbb{Z} / m(1)\right)$ induced by the Kummer sequence. In the second pairing $(,)_{2}$, the restriction to $H^{1}\left(D_{1}, \mathbb{Z} / m\right) \times \Gamma\left(D_{1}, \mathbb{G}_{m}\right)$ is trivial. Hence 
the pairing annihilates $\tilde{A}^{2}$ and the pairing $():, H^{1}(U, \mathbb{Z} / m) \times A \rightarrow H^{1}(T, \mathbb{Z} / m)$ is defined.

Using the tame symbol defined above, we obtain a local pairing

$$
(,)_{x}: H^{1}(U, \mathbb{Z} / m) \times A_{x} \rightarrow H^{1}(x, \mathbb{Z} / m)
$$

as in Section 3. To define a pairing $(,)_{X}: H^{1}(U, \mathbb{Z} / m) \times C H^{n}(X \bmod D) \rightarrow$ $H^{1}(k, \mathbb{Z} / m)$, it is sufficient to show the reciprocity law that the pairing

$$
\bigoplus_{x} \operatorname{Tr}_{x / k} \circ(,)_{x}: H^{1}(U, \mathbb{Z} / m) \times \bigoplus A_{x} \rightarrow H^{1}(k, \mathbb{Z} / m)
$$

annihilates the image of the boundary map $\partial: \bigoplus_{y \in X_{1}} B_{y} \rightarrow \bigoplus_{x \in X_{0}} A_{x}$ by Proposition 1 in Section 2.

We prove the reciprocity for higher dimension as in the proof of Proposition 2 in Section 3. Let $D_{i}$ be an irreducible component of $D, k_{i}$ be the constant field of $D_{i}$ and $\partial_{i}: H^{1}(U, \mathbb{Z} / m) \rightarrow H^{0}\left(D_{i}, \mathbb{Z} / m(-1)\right)=H^{0}\left(k_{i}, \mathbb{Z} / m(-1)\right)$ be the boundary map. Then for a closed point $x \in D_{i}$, the restriction of the pairing to the $i$-th component $\kappa(x)^{\times} \subset A_{x, i} \cap A_{x}$ factors as

$$
\begin{aligned}
H^{1}(U, \mathbb{Z} / m) \times \kappa(x)^{\times} & \stackrel{\partial_{i} \times\left(\kappa \circ N_{x / k_{i}}\right)}{\longrightarrow} H^{0}\left(k_{i}, \mathbb{Z} / m(-1)\right) \times H^{1}\left(k_{i}, \mu_{m}\right) \\
& \stackrel{\cup}{\longrightarrow} H^{1}\left(k_{i}, \mathbb{Z} / m\right) .
\end{aligned}
$$

Hence the pairing annihilates the kernel of the norm $\bigoplus_{x \in\left(D_{i}\right)_{0}} \kappa(x)^{\times} \rightarrow k_{i}^{\times}$. Since the composition $\bigoplus_{y \in\left(D_{i}\right)_{1}} K_{2}(y) \rightarrow \bigoplus_{x \in\left(D_{i}\right)_{0}} \kappa(x)^{\times} \rightarrow k_{i}^{\times}$is the 0-map, the restriction of the pairing $(, \partial)_{X}$ to the kernel of $B_{y} \rightarrow \kappa(y)^{\times}$is trivial for $y \in X_{1}$. Therefore it is sufficient to show that for each $y \in X_{1}$ and $f \in \kappa(y)^{\times}$, there is a lifting $\tilde{f} \in B_{y}$ of $f$ such that the pairing $(, \partial \tilde{f})$ is trivial.

Let $Y$ be the closure of $y$ and $\tilde{Y}$ be the normalization of $Y$, and let $f \in \kappa(y)^{\times}$. We take an open subset $W \subset Y$ containing the singularities of $Y$, the intersection $Y \cap D_{i}$ for $i \notin I_{y}=\left\{i \in I ; y \notin D_{i}\right\}$ and the zeroes and poles of $f$ and take a family $\pi$ of bases $\pi_{i}$ of $\left.\mathcal{O}_{Y}\left(-D_{i}\right)\right|_{W}$ for $i \in I_{y}$. Let $\tilde{W}^{*}$ be the inverse image of $W-\bigcup_{i \in I-I_{y}} D_{i}$ in $\tilde{Y}$. The tame symbol $(, \pi)$ defines a map $H^{1}(U, \mathbb{Z} / m) \rightarrow H^{1}\left(\tilde{W}^{*}, \mathbb{Z} / m\right)$. Similarly as loc.cit., it is sufficient to show the equality of the map

$$
((, \pi), \partial f)_{\tilde{Y}}=(, \partial\{\pi, f\})_{X}: H^{1}(U, \mathbb{Z} / m) \rightarrow H^{1}(k, \mathbb{Z} / m) .
$$

It follows from the local equality

$$
\left((, \pi), \partial_{\tilde{x}} f\right)_{\tilde{x}}=\left(, \partial_{\tilde{x}, y}\{\pi, f\}\right)_{\tilde{x}}: H^{1}(U, \mathbb{Z} / m) \rightarrow H^{1}(\tilde{x}, \mathbb{Z} / m)
$$

for $\tilde{x} \in \tilde{Y}$.

First we assume the image $x$ of $\tilde{x}$ is in $W$. By replacing $X$ by an etale neighborhood $X^{\prime}$ with $x \in X^{\prime} \rightarrow X$, we may assume $H^{1}(U, \mathbb{Z} / m)$ is generated by $\bigoplus_{i \in I_{x}} H^{1}\left(X-D_{i}, \mathbb{Z} / m\right)$. Hence we may assume Card $I_{x} \leq 1$. If $I_{x}=\emptyset$, both sides $\left((, \pi), \partial_{\tilde{x}} f\right)$ and $\left(, \partial_{\tilde{x}, y}\{\pi, f\}\right)$ are simply $\operatorname{ord}_{\tilde{x}} f \cdot\left(\left.\right|_{\tilde{x}}\right): H^{1}(X, \mathbb{Z} / m) \rightarrow H^{1}(\tilde{x}, \mathbb{Z} / m)$. We assume Card $I_{x}=1$ and let 0 be the unique element and $\pi_{0}$ be a prime element of $D=D_{0}$ at $x$. First assume $y \in D=D_{0}$. We may assume $\pi=\left(\pi_{0}\right)$. Then they are equal to $\left.\operatorname{ord}_{\tilde{x}} f \cdot\left(, \pi_{0}\right)\right|_{\tilde{x}}: H^{1}(U, \mathbb{Z} / m) \rightarrow H^{1}(\tilde{x}, \mathbb{Z} / m)$. Finally assume $y \notin D$. Then $I_{y}=\emptyset, \pi$ is the empty family and $(\chi, \pi)=\left.\chi\right|_{\tilde{W}^{*}}$. We have an exact sequence

$$
0 \rightarrow H^{1}(X, \mathbb{Z} / m) \rightarrow H^{1}(U, \mathbb{Z} / m) \rightarrow H^{0}(D, \mathbb{Z} / m(-1))
$$


and the assertion is proved for $H^{1}(X, \mathbb{Z} / m)$. Hence by localizing if necessary, we may assume $\mathbb{Z} / m(1) \simeq \mathbb{Z} / m$ on $X$ and $\chi=\kappa\left(\pi_{0}\right) \in H^{1}(U, \mathbb{Z} / m) \simeq H^{1}(U, \mathbb{Z} / m(1))$. Then the left hand side is $\left(\left.\chi\right|_{\tilde{W}^{*}}, \partial_{\tilde{x}} f\right)=\kappa\left\{\pi_{0}, f\right\}_{\tilde{x}} \in H^{1}(\tilde{x}, \mathbb{Z} / m)$. We compute the right hand side. By the explicit computation $\partial_{\tilde{x}, y} f=\left(-\pi_{0}\right)^{\operatorname{ord}_{\tilde{x}} f} \cdot\left\{\pi_{0}^{-1}, f\right\}_{\tilde{x}}$, we have

$$
\left(\chi, \partial_{\tilde{x}, y} f\right)=\kappa\left(\left\{\pi_{0},-\pi_{0}\right\}^{\operatorname{ord}_{\tilde{x}} f} \cdot\left\{\pi_{0},\left\{\pi_{0}^{-1}, f\right\}_{\tilde{x}}\right\}\right)=\kappa\left(\left\{\pi_{0}, f\right\}_{\tilde{x}}\right) .
$$

Thus the local equality is proved if $x \in W$.

We assume $x \notin W$. We take a local basis $\pi_{i}^{\prime}$ of $\left.N_{i}\right|_{Y}$ at $x$ for $i \in I_{y}=I_{x}$ and put $\pi_{i}=u_{i} \cdot \pi_{i}^{\prime}, u_{i} \in \kappa(y)^{\times}$. Then for $\chi \in H^{1}(U, \mathbb{Z} / m)$, we have $(\chi, \pi)=$ $\left(\chi, \pi^{\prime}\right)+\sum_{i} \partial_{i} \chi \cup \kappa\left(u_{i}\right)$ and $\left(\chi, \pi^{\prime}\right)$ is unramified at $x$. Since $f$ is a unit at $x$, we have $\left(\left(\chi, \pi^{\prime}\right), \partial_{x} f\right)=0$ and

$$
\begin{aligned}
& \left((\chi, \pi), \partial_{x} f\right)=\sum_{i}\left(\partial_{i} \chi \cup \kappa\left(u_{i}\right), \partial_{x} f\right) \\
= & \sum_{i} \partial_{i} \chi \cup \kappa\left(\left\{u_{i}, f\right\}_{x}\right)=-\sum_{i} \operatorname{ord}_{x} u_{i} \cdot \partial_{i} \chi \cup \kappa(f(x)) .
\end{aligned}
$$

On the other hand, by $\partial_{x, y}\{\pi, f\}=\left(f^{-\operatorname{ord}_{x} u_{i}}\right)_{i} \in \bigoplus_{i} \kappa(x)^{\times} \subset A_{x}$, the left hand side $\left(\chi, \partial_{x, y}\{\pi, f\}\right)$ is also $-\sum_{i} \operatorname{ord}_{x} u \cdot \partial_{i} \chi \cup \kappa(f(x))$. Thus the equality is proved for $x \notin W$. Thus the proof of Proposition 3 is completed.

In the rest of the paper, we assume the Gersten conjecture for K-theory holds for a discrete valuation ring. By $[\mathrm{G}]$, it implies the Gersten conjecture for a smooth scheme over a discrete valuation ring.

Proposition 4. Let $X$ be a proper smooth scheme over a regular noetherian connected scheme $S$ and $D$ be a divisor of $X$ with simple normal crossings relative to $S$. Then there exists a unique pairing

$$
\pi_{1}(S)^{\mathrm{ab}} \times C H^{n}(X \bmod D) \rightarrow \pi_{1}(U)^{\mathrm{ab}, \text { tame }}
$$

such that for a point $s \in S$, the diagram

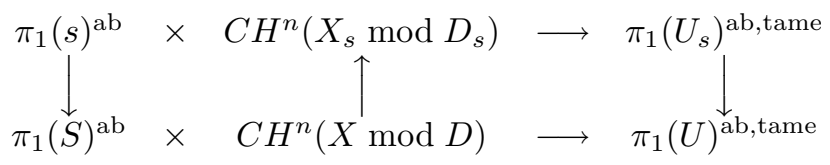

is commutative.

Proof. The uniqueness follows from the surjectivity of $\pi_{1}(\eta)^{\mathrm{ab}} \rightarrow \pi_{1}(S)^{\mathrm{ab}}$ for the generic point $\eta$ of $S$. We show the existence. We show the pairing $\pi_{1}(\eta)^{\text {ab }} \times$ $C H^{n}(X \bmod D) \rightarrow \pi_{1}(\eta)^{\mathrm{ab}} \times C H^{n}\left(X_{\eta} \bmod D_{\eta}\right) \rightarrow \pi_{1}\left(U_{\eta}\right)^{\mathrm{ab} \text {,tame }} \rightarrow \pi_{1}(U)^{\text {ab,tame }}$ factors the quotient $\pi_{1}(S)^{\mathrm{ab}}$. In other words, the image of the cup-product

$$
H^{1}(U, \mathbb{Q} / \mathbb{Z})^{\text {tame }} \times C H^{n}(X \bmod D) \rightarrow H^{1}(\eta, \mathbb{Q} / \mathbb{Z})
$$

is in $H^{1}(S, \mathbb{Q} / \mathbb{Z})$. By the purity of the branch locus, we may assume $S$ is the spectrum of a discrete valuation ring. Let $K$ be the fraction field and $F$ be the residue field of $S$.

For the $p$-part where $p=\operatorname{char} F$, it is Proposition 1 of [S2]. Let $m$ be an integer invertible in $F$ and consider the pairing

$$
H^{1}(U, \mathbb{Z} / m) \times C H^{n}\left(X_{\eta} \bmod D_{\eta}\right) \rightarrow H^{1}(\eta, \mathbb{Z} / m) .
$$


By the Gersten resolution, we have an isomorphism

$$
H_{X F}^{n}\left(X, \mathcal{K}_{n}(X \bmod D)\right) \rightarrow \bigoplus_{i} H^{n-1}\left(D_{i, F}, \mathcal{K}_{n-1}\left(D_{i, F}\right)\right)=\bigoplus_{i} C H^{n-1}\left(D_{i, F}\right)
$$

and an exact sequence

$$
C H^{n}(X \bmod D) \rightarrow C H^{n}\left(X_{K} \bmod D_{K}\right) \stackrel{\partial}{\rightarrow} \bigoplus_{i} C H^{n-1}\left(D_{i, F}\right) .
$$

The ramification theory gives a map $\partial_{i}: H^{1}(U, \mathbb{Z} / m) \rightarrow H^{0}\left(D_{i}, \mathbb{Z} / m(-1)\right)$ for a irreducible component $D_{i}$ and $\partial: H^{1}(K, \mathbb{Z} / m) \rightarrow H^{0}(F, \mathbb{Z} / m(-1))$. Since Ker $\partial=$ $H^{1}(S, \mathbb{Z} / m)$, the following Claim implies the existence of a pairing

$$
H^{1}(U, \mathbb{Z} / m) \times C H^{n}(X \bmod D) \rightarrow H^{1}(S, \mathbb{Z} / m)
$$

Claim 1. The diagram

$$
\begin{aligned}
H^{1}(U, \mathbb{Z} / m) \times C H^{n}\left(X_{K} \bmod D_{K}\right) & H^{1}(K, \mathbb{Z} / m) \\
\left(\oplus_{i} \partial_{i}\right) \times \partial \downarrow & \downarrow \partial \\
\bigoplus_{i} H^{0}\left(D_{i, F}, \mathbb{Z} / m(-1)\right) \times \bigoplus_{i} C H^{n-1}\left(D_{i, F}\right) & \longrightarrow H^{0}(F, \mathbb{Z} / m(-1))
\end{aligned}
$$

is commutative. Here the lower pairing is the sum of

$$
\begin{aligned}
H^{0}\left(D_{i, F}, \mathbb{Z} / m(-1)\right) & \times C H^{n-1}\left(D_{i, F}\right) \stackrel{\text { can } \times \operatorname{deg}_{F}}{\longrightarrow} H^{0}\left(F_{i}, \mathbb{Z} / m(-1)\right) \\
& \stackrel{\operatorname{Tr}_{F_{i} / F}}{\longrightarrow} H^{0}(F, \mathbb{Z} / m(-1))
\end{aligned}
$$

where $F_{i}$ is the constant field of $D_{i, F}$.

We prove Claim 1 later and assume a pairing is defined. We show the commutativity of the diagram in Proposition 4 for an arbitrary point $s$ of $S$. By considering the blowing-up at the closure of $s$ and by the same argument as in the proof of Proposition 1 in [S2], we may assume that the codimension of $s$ is 1 . Hence we may assume $S$ is the spectrum of a discrete valuation ring and $s$ is the closed point. For the $p=$ char $F$-part, it is proved in loc.cit. Let $m$ be an integer invertible in $F$. We take a prime element $\pi$ of $K$. Let $s p_{\pi}$ denote the composition

$$
\begin{gathered}
C H^{n}\left(X_{K} \bmod D_{K}\right) \stackrel{\{, \pi\}}{\longrightarrow} H^{n}\left(X_{K}, \mathcal{K}_{n+1}\left(X_{K} \bmod D_{K}\right)\right) \\
\stackrel{\partial}{\longrightarrow} H_{X_{F}}^{n+1}\left(X, \mathcal{K}_{n+1}(X \bmod D)\right) \\
\simeq H^{n}\left(X_{F}, \mathcal{K}_{n}\left(X_{F} \bmod D_{F}\right)\right)=C H^{n}\left(X_{F} \bmod D_{F}\right)
\end{gathered}
$$

and also

$$
H^{1}(K, \mathbb{Z} / m) \stackrel{\cup\{\pi\}}{\longrightarrow} H^{2}(K, \mathbb{Z} / m(1)) \rightarrow H^{1}(F, \mathbb{Z} / m) .
$$

The restriction to the closed fiber res : $C H^{n}(X \bmod D) \rightarrow C H^{n}\left(X_{F} \bmod D_{F}\right)$ is equal to the composition

$$
C H^{n}(X \bmod D) \rightarrow C H^{n}\left(X_{K} \bmod D_{K}\right) \stackrel{s p_{\pi}}{\longrightarrow} C H^{n}\left(X_{F} \bmod D_{F}\right)
$$

and the restriction $H^{1}(S, \mathbb{Z} / m) \rightarrow H^{1}(F, \mathbb{Z} / m)$ is

$$
H^{1}(S, \mathbb{Z} / m) \rightarrow H^{1}(K, \mathbb{Z} / m) \stackrel{s p_{\pi}}{\longrightarrow} H^{1}(F, \mathbb{Z} / m) .
$$

Hence it is sufficient to show the following. 
Claim 2. The diagram

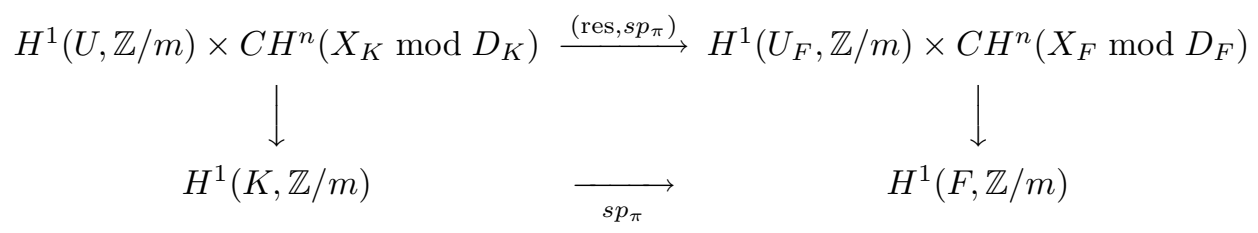

is commutative.

We deduce Claims 1 and 2 from

Claim 3. Let $y$ be a closed point of $U_{K}$ and $Y$ be the closure of $\{y\}$ in $X$. Then

(1) The image of the class $[y]$ under

$$
\partial: C H^{n}\left(X_{K} \bmod D_{K}\right) \rightarrow \bigoplus_{i} C H^{n-1}\left(D_{i, F}\right)
$$

is $\partial[y]=\left(\left(D_{i}, Y\right)\right)_{i} \in \bigoplus_{i} C H^{n-1}\left(D_{i, F}\right)$. Here $\left(D_{i}, Y\right)$ is the intersection product $\sum_{x \in Y \cap D_{i}}$ length $\mathcal{O}_{Y \cap D_{i}, x} \cdot[x]$.

(2) For a closed point $x$ of $Y_{0}=Y \cap X_{F}$, let $\partial_{x, y}$ denote the map $\kappa(y)^{\times} \rightarrow A_{x}$ defined in Section 2. Then the diagram

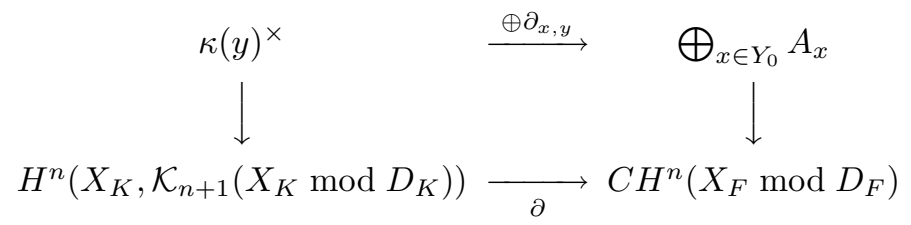

is commutative.

We show Claim 3 implies Claims 1 and 2 and hence Proposition 4.

Proof of Claim 1. Let $\chi \in H^{1}(U, \mathbb{Z} / m)$ and $y$ be a closed point of $U_{K}$. For a closed point $\tilde{x}$ of the normalization $\tilde{Y}$ of $Y$, the boundary $\partial_{\tilde{x}}\left(\left.\chi\right|_{y}\right) \in H^{0}(\tilde{x}, \mathbb{Z} / m(-1))$ is given by $\left.\sum_{i} \operatorname{ord}_{\tilde{x}}\left(\left.D_{i}\right|_{\tilde{Y}}\right) \cdot\left(\partial_{i} \chi\right)\right|_{\tilde{x}}$. Hence

$$
\partial(\chi,[y])=\partial\left(\operatorname{Tr}_{y / K}\left(\left.\chi\right|_{y}\right)\right)=\sum_{x \in \tilde{Y}_{0}} \operatorname{Tr}_{\tilde{x} / F} \partial_{\tilde{x}}\left(\left.\chi\right|_{y}\right)
$$

is equal to $\sum_{i} \sum_{\tilde{x}}\left[\kappa(\tilde{x}): F_{i}\right] \cdot\left(\operatorname{ord}_{\tilde{x}}\left(\left.D_{i}\right|_{\tilde{Y}}\right)\right) \cdot \operatorname{Tr}_{F_{i} / F}\left(\partial_{i} \chi\right)$. Since $\sum_{\tilde{x}}\left[\kappa(\tilde{x}): F_{i}\right]$. $\operatorname{ord}_{\tilde{x}}\left(\left.D_{i}\right|_{\tilde{Y}}\right)=\operatorname{deg}_{F_{i}}\left(D_{i}, Y\right)$, we have $\partial(\chi,[y])=\sum_{i}\left(\partial_{i} \chi,\left(D_{i}, Y\right)\right)$. Since the right hand side is $\left(\sum_{i} \partial_{i} \chi, \partial[y]\right)$ by Claim $3(1)$ and $C H^{n}\left(X_{K} \bmod D_{K}\right)$ is generated by the class of closed points of $U_{K}$, Claim 1 is proved.

Proof of Claim 2. We use the above notation. By Claim 3 (2), we have $\left(\chi, s p_{\pi}[y]\right)=$ $\sum_{x \in Y_{0}} \operatorname{Tr}_{x / F}\left(\chi, \partial_{x, y}(\pi)\right)_{x}$. On the other hand, we have $s p_{\pi}(\chi,[y])=\partial_{F} \operatorname{Tr}_{y / K}(\chi, \pi)$ $=\sum_{\tilde{x} \in \tilde{Y}_{0}} \operatorname{Tr}_{\tilde{x} / F}(\chi, \pi)_{\tilde{x}}$. Hence it is sufficient to show $\left(\chi, \partial_{\tilde{x}, y}(\pi)\right)_{\tilde{x}}=(\chi, \pi)_{\tilde{x}}$. This is proved by exactly the same argument as the equality $\left(\chi, \partial_{\tilde{x}, y}(\{\pi, f\})\right)_{\tilde{x}}=$ $\left((\chi, \pi), \partial_{\tilde{x}} f\right)$ in the proof of the reciprocity law in Proposition 3. 
Proof of Claim 3. The proof is similar to that for Proposition 1 given in the Appendix. It is sufficient to prove that the boundary maps

$$
\begin{gathered}
\mathbb{Z}=H_{y}^{n}\left(X, \mathcal{K}_{n}(X \bmod D)\right) \rightarrow H_{x}^{n+1}\left(X, \mathcal{K}_{n}(X \bmod D)\right)=\bigoplus_{D_{i} \ni x} \mathbb{Z}, \\
\kappa(y)^{\times}=H_{y}^{n}\left(X, \mathcal{K}_{n+1}(X \bmod D)\right) \rightarrow H_{x}^{n+1}\left(X, \mathcal{K}_{n+1}(X \bmod D)\right)=A_{x}
\end{gathered}
$$

are given by $1 \mapsto\left(\left(D_{i}, Y\right)_{x}\right)_{i}$ and $\partial_{x, y}$ respectively. The second one is proved by the same argument as loc.cit. and the first one is similar and easier. The only point to be proved is the technical Claim 1 loc.cit. However, it is proved by taking etale locally a smooth projection $X \rightarrow D$ over $S$ by the same argument. The rest is proved in the same way and we leave the details to the reader.

(b) Jacobi sum and determinant of $\ell$-adic cohomology. Using Propositions 3 and 4 above, we have a variant of Theorem 1 in [S2] for the relative canonical class in the relative Chow group. To state it, we review Jacobi sums following [A]. Let $k$ be a field of characteristic $p \geq 0$ and $F$ be a field of characteristic 0 . Let $\bar{k}$ and

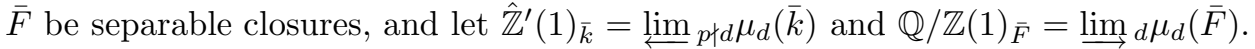
Let $B_{F}(k)$ be the free abelian group with basis $\operatorname{Hom}\left(\hat{\mathbb{Z}}^{\prime}(1)_{\bar{k}}, \mathbb{Q} / \mathbb{Z}(1)_{\bar{F}}\right)$ and $B_{F}^{0}(k)$ be the kernel of the natural map $B_{F}(k) \rightarrow \operatorname{Hom}\left(\hat{\mathbb{Z}}^{\prime}(1)_{\bar{k}}, \mathbb{Q} / \mathbb{Z}(1)_{\bar{F}}\right)$. If we choose an isomorphism $\hat{\mathbb{Z}}^{\prime}(1)_{\bar{k}} \simeq \hat{\mathbb{Z}}^{\prime}(1)_{\bar{F}}$, the group $\operatorname{Hom}\left(\hat{\mathbb{Z}}^{\prime}(1)_{\bar{k}}, \mathbb{Q} / \mathbb{Z}(1)_{\bar{F}}\right)$ is naturally identified with $(\mathbb{Q} / \mathbb{Z})^{\prime}=\operatorname{inj} \lim _{p \nmid d} \mathbb{Z} / d$ and there are inclusions $B_{F}(k) \rightarrow \mathbb{B}$ and $B_{F}^{0}(k) \rightarrow \mathbb{B}^{0}$. Here the free abelian group $\mathbb{B}$ with basis $\mathbb{Q} / \mathbb{Z}$ and $\mathbb{B}^{0}=\operatorname{Ker} \mathbb{B} \rightarrow \mathbb{Q} / \mathbb{Z}$ are as in [A]. The groups $B_{F}(k)$ and $B_{F}^{0}(k)$ have natural actions of the absolute Galois groups $G_{k}=\operatorname{Gal}(\bar{k} / k)$ and $G_{F}=\operatorname{Gal}(\bar{F} / F)$. The automorphism group of $\operatorname{Hom}\left(\hat{\mathbb{Z}}^{\prime}(1)_{\bar{k}}, \mathbb{Q} / \mathbb{Z}(1)_{\bar{F}}\right)$ as an abstract group is $\hat{\mathbb{Z}}^{\prime \times}=\varliminf_{p \nmid d}(\mathbb{Z} / d)^{\times}$. The action is given by the cyclotomic characters $G_{k} \rightarrow \hat{\mathbb{Z}}^{\prime \times}$ and $G_{F} \rightarrow \hat{\mathbb{Z}}^{\prime \times}$.

We define the Jacobi sum $J(\mathbf{a})$ for an element $\mathbf{a} \in B_{F}^{0}(k)^{G_{k} \times G_{F}}$ fixed by the actions of $G_{k}$ and $G_{F}$. First we consider the case where $k$ is a finite field of order $q$. The subgroup $B_{F}(k)^{G_{k}}$ of $B_{F}(k)$ consisting of the elements fixed by $G_{k}$ is generated by the elements of the form $\mathbf{a}=\sum_{i=0}^{f-1}\left[q^{i} a\right]$. Here the order $m(a)$ of $a \in$ $\operatorname{Hom}\left(\hat{\mathbb{Z}}^{\prime}(1)_{\bar{k}}, \mathbb{Q} / \mathbb{Z}(1)_{\bar{F}}\right)$ is prime to $q$ and $f$ is the order of $q$ in $(\mathbb{Z} / m(a))^{\times}$. For a non-trivial additive character $\psi_{0}: k \rightarrow \bar{F}^{\times}$, we define a map $g\left(, \psi_{0}\right): B_{F}(k)^{G_{k}} \rightarrow$ $\bar{F}^{\times}$by

$$
g\left(\mathbf{a}, \psi_{0}\right)=-\sum_{x \in E_{f}^{\times}} a^{-1}(x) \psi_{0}\left(\operatorname{Tr}_{E_{f} / k} x\right)
$$

for an element $\mathbf{a}=\sum_{i=0}^{f-1}\left[q^{i} a\right]$ as above. Here $E_{f}$ is the extension of $k$ of degree $f$ in $\bar{k}$ and $a$ is regarded as a character of $E_{f}^{\times}$by $E_{f}^{\times}=\mu_{q^{f}-1}(\bar{k})$. The restriction $J_{k}$ of $g\left(, \psi_{0}\right)$ to $B_{F}^{0}(k)^{G_{k} \times G_{F}}$ is independent of the choice of $\psi_{0}$ and the image is in $F^{\times}$. Hence $J_{k}$ is a map $B_{F}^{0}(k)^{G_{k} \times G_{F}} \rightarrow F^{\times}$.

We consider a general field $k$. Let $\mathbf{a}=\sum_{a} n_{a}[a] \in B_{F}^{0}(k)$ be an element fixed by $G_{k}$ and $G_{F}$, and let $m(\mathbf{a})$ denote the order of the subgroup of $\operatorname{Hom}\left(\hat{\mathbb{Z}}^{\prime}(1)_{\bar{k}}, \mathbb{Q} / \mathbb{Z}(1)_{\bar{F}}\right)$ generated by $\left\{a ; n_{a} \neq 0\right\}$. We define a subfield $F_{\mathbf{a}}$ of $F \subset \mathbb{Q}\left(\zeta_{m(\mathbf{a})}\right)$. Let $G_{\mathbf{a}}$ be the subgroup of $(\mathbb{Z} / m(\mathbf{a}))^{\times}$fixing a. We define $F_{\mathbf{a}}$ to be the subfield of $\mathbb{Q}\left(\zeta_{m(\mathbf{a})}\right) \subset \bar{F}$ fixed by the subgroup $G_{\mathbf{a}}$ of $(\mathbb{Z} / m(\mathbf{a}))^{\times}=\operatorname{Gal}\left(\mathbb{Q}\left(\zeta_{m(\mathbf{a})}\right) / \mathbb{Q}\right)$. Since $G_{\mathbf{a}}$ includes the image of the cyclotomic character $G_{F} \rightarrow(\mathbb{Z} / m(\mathbf{a}))^{\times}$, it is a subfield of $F$. 
Take an isomorphism $\hat{\mathbb{Z}}^{\prime}(1)_{\bar{k}} \simeq \hat{\mathbb{Z}}^{\prime}(1)_{\bar{F}}$. Then the image of $\mathbf{a} \in B_{F}^{0}(k)$ in $\mathbb{B}^{0}$ is fixed by $G_{F_{\mathbf{a}}}$ and defines an algebraic Hecke character $J(\mathbf{a})$ of $F_{\mathbf{a}}$ with values in $F_{\mathbf{a}}$ with conductor dividing a power of $m(\mathbf{a})$ by $[\mathrm{A}]$. Since $G_{\mathbf{a}}$ also includes the image of $G_{k}$, the isomorphism $\hat{\mathbb{Z}}^{\prime}(1)_{\bar{k}} \simeq \hat{\mathbb{Z}}^{\prime}(1)_{\bar{F}}$ chosen induces a homomorphism $\mathcal{O}_{F_{\mathbf{a}}}[1 / m(\mathbf{a})] \rightarrow k$ and a homomorphism $G_{k}^{\mathrm{ab}} \rightarrow \pi_{1}\left(\mathcal{O}_{F_{\mathbf{a}}}[1 / m(\mathbf{a})]\right)^{\text {ab }}$. If $F$ is a finite extension of $\mathbb{Q}_{\ell}$ for a prime $\ell \neq p$, the algebraic Hecke character $J(\mathbf{a})$ induces a character $\pi_{1}\left(\mathcal{O}_{F_{\mathbf{a}}}[1 / \ell m(\mathbf{a})]\right)^{\text {ab }} \rightarrow F^{\times}$. The composite character $J(\mathbf{a}): G_{k}^{\mathrm{ab}} \rightarrow F^{\times}$is independent of the choice of an isomorphism $\hat{\mathbb{Z}}^{\prime}(1)_{\bar{k}} \simeq \hat{\mathbb{Z}}^{\prime}(1)_{\bar{F}}$. If $k$ is a finite field, the value of the geometric Frobenius $F r_{k}$ is given by $J(\mathbf{a})\left(F r_{k}\right)=J_{k}(\mathbf{a}) \in F^{\times}$. Summing up, we have defined the Jacobi sums in the following sense.

Definition 10. Let $k$ be a field, $F$ a field of characteristic 0 and $\mathbf{a} \in B_{F}^{0}(k)^{G_{k} \times G_{F}}$.

(1) If $k$ is finite, the Jacobi sum $J_{k}(\mathbf{a}) \in F^{\times}$is a certain product of the Gauss sums defined by the formula (1), and is defined as the image of a defined by the map $J_{k}: B_{F}^{0}(k)^{G_{k} \times G_{F}} \rightarrow F^{\times}$.

(2) Assume $k=F=F_{\mathbf{a}}$ is the subfield of $\mathbb{Q}\left(\zeta_{m(\mathbf{a})}\right)$ defined as above. Then the Jacobi sum $J(\mathbf{a})$ is the algebraic Hecke character of $k$ with values in $F$ such that, for finite places $v \nmid m(\mathbf{a})$ of $k, J(\mathbf{a})(v)=J_{k(v)}(\mathbf{a}) \in F^{\times}$.

(3) Assume char $k \neq \ell$ and $F$ is a finite extension of $\mathbb{Q}_{\ell}$. Then the algebraic Hecke character $J(\mathbf{a})$ in $(2)$ defines a character $\pi_{1}\left(O_{F_{\mathbf{a}}}[1 / \ell m(\mathbf{a})]\right)^{\text {ab }} \rightarrow F^{\times}$ and the Jacobi sum $J(\mathbf{a}): G_{k}^{\mathrm{ab}} \rightarrow F^{\times}$is the composition of the map above with the natural map $G_{k}^{\mathrm{ab}} \rightarrow \pi_{1}\left(O_{F_{\mathbf{a}}}[1 / \ell m(\mathbf{a})]\right)^{\mathrm{ab}}$.

We return to a geometric situation. Let $X$ be a proper smooth scheme over a field $k$ of characteristic $p \geq 0$ and $U$ be an open subscheme of $X$ whose complement $D$ is a divisor with simple normal crossings. Let $F_{\lambda}$ be a finite extension of $\mathbb{Q}_{\ell}$, for $\ell \neq p$, and $V_{\lambda}$ be a smooth $F_{\lambda}$-sheaf on $U^{e t}$ of rank $r$. We assume that the ramification of $V_{\lambda}$ along $D$ is tame and the local monodromy at each irreducible component $D_{i}$ is quasi-unipotent. The assumption is automatically satisfied if $k$ is a number field by the monodromy theorem of Grothendieck.

We define an element $\mathbf{a}_{D, V_{\lambda}} \in B_{F_{\lambda}}^{0}(k)$ fixed by $G_{k}$ and $G_{F}$ and a character $J_{D, V_{\lambda}}=J\left(\mathbf{a}_{D, V_{\lambda}}\right): G_{k}^{\mathrm{ab}} \rightarrow F_{\lambda}^{\times}$. Let $k_{i}$ be the constant field of an irreducible component $D_{i}$. Since the ramification of $V_{\lambda}$ is tame, the local monodromy defines a continuous representation $\rho_{i}: \hat{\mathbb{Z}}^{\prime}(1)_{\bar{k}_{i}} \rightarrow G L_{r}\left(F_{\lambda}\right)$. Since it is assumed to be quasi-unipotent, its semi-simplification $\rho_{i}^{s s}$ is the direct sum $\bigoplus_{j=1}^{r} a_{i j}$ of characters $a_{i j}: \hat{\mathbb{Z}}^{\prime}(1)_{\bar{k}_{i}} \rightarrow \mathbb{Q} / \mathbb{Z}(1)_{\bar{F}_{\lambda}}$. We put $\mathbf{a}_{i}=\sum_{j=1}^{r}\left[a_{i j}\right] \in B_{F_{\lambda}}\left(k_{i}\right)$. It is fixed by $G_{k_{i}}$ and $G_{F_{\lambda}}$.

Definition 11. Let $V_{\lambda}$ be a smooth $F_{\lambda}$-sheaf on $U^{\text {et }}$ of rank $r$ such that the ramification of $V_{\lambda}$ along $D$ is tame and the local monodromy at each irreducible component $D_{i}$ is quasi-unipotent.

(1) We define an element $\mathbf{a}_{D, V_{\lambda}} \in B_{F_{\lambda}}^{0}(k)^{G_{k} \times G_{F}}$ to be $\sum_{i} c_{i} \operatorname{Tr}_{k_{i} / k}\left(\mathbf{a}_{i}\right)$. Here $c_{i}$ is the Euler number of $D_{i}^{*} \otimes_{k_{i}} \bar{k}_{i}$ where $D_{i}^{*}=D_{i}-\bigcup_{j \neq i} D_{j}$ and $\mathbf{a}_{i}$ is defined by the semi-simplification $\rho_{i}^{s s}$ as above.

(2) We define a character $J_{D, V_{\lambda}}: G_{k}^{\mathrm{ab}} \rightarrow F_{\lambda}^{\times}$as $J\left(\mathbf{a}_{D, V_{\lambda}}\right)$ (Definition 10(3)).

It is clear that $\mathbf{a}_{D, V_{\lambda}}$ is fixed by $G_{k}$ and $G_{F_{\lambda}}$. By the first assertion of Theorem 1 in $[\mathrm{S} 2]$, it is in $B_{F_{\lambda}}^{0}(k)$.

Now we state a variant of Theorem 1 in [S2] for the relative Chow group. Let $V_{\lambda}$ be a smooth $F_{\lambda}$-sheaf of rank $r$ on a smooth scheme $U$ over a field $k$, 
where $F_{\lambda}$ is a finite extension of $\mathbb{Q}_{\ell}, \ell \neq$ char $k$. We define an $F_{\lambda}$-representation $\left(\operatorname{det} V_{\lambda}, c_{X \bmod D}\right)$ of dimension 1 of $G_{k}$ to be the pull-back of the $F_{\lambda}$-representation $\wedge^{r} V_{\lambda}$ of $\pi_{1}(U)^{\text {ab,tame }}$ by the pairing $\left(, c_{X \bmod D}\right): G_{k} \rightarrow \pi_{1}(U)^{\text {ab,tame }}$ defined in Proposition 3 with the relative canonical class $c_{X \bmod D}$ defined in Definition 6 in Section 2.

Theorem S. Let $U$ be a smooth scheme over a field $k, F_{\lambda}$ be a finite extension of $\mathbb{Q}_{\ell}$ where $\ell \neq$ char $k$ and $V_{\lambda}$ be a smooth $F_{\lambda}$-sheaf of rank $r$ on $U$ as above. Assume the following conditions (1)-(3) are satisfied.

(1) There exists a projective smooth scheme $X$ over $k$ including $U$ as the complement of a divisor $D$ with relative normal crossings.

(2) The ramification of $V_{\lambda}$ along $D$ is tame.

(3) There exists a scheme $S_{0}$ of finite type over $\mathbb{Z}$ and an $S_{0}$-scheme $U_{0}$ such that $U=U_{0} \times_{S_{0}}$ Spec $k$ and $V_{\lambda}$ is a pull-back of a smooth $F_{\lambda}$-sheaf on $U_{0}$.

Then the local monodromies are quasi-unipotent and the character $J_{D, V_{\lambda}}: G_{k}^{\mathrm{ab}} \rightarrow$ $F_{\lambda}^{\times}$is defined. Assume also the Gersten conjecture for discrete valuation rings. Then there is an isomorphism of $F_{\lambda}$-representations of dimension 1 of $G_{k}$

$$
\operatorname{det} R \Gamma_{c}\left(U_{\bar{k}}, V_{\lambda}\right) \otimes\left(\operatorname{det} R \Gamma_{c}\left(U_{\bar{k}}, F_{\lambda}\right)\right)^{\otimes-r} \simeq\left(\operatorname{det} V_{\lambda}, c_{X \bmod D}\right) \otimes J_{D, V_{\lambda}}^{\otimes-1} .
$$

Here the $F_{\lambda}$-representations $\left(\operatorname{det} V_{\lambda}, c_{X \bmod D}\right)$ and $J_{D, V_{\lambda}}^{\otimes-1}$ of $G_{k}^{\mathrm{ab}}$ of dimension 1 are defined above and in Definition 11.

Proof. By the assumption (3), the first assertion is an immediate consequence of the monodromy theorem of Grothendieck. The isomorphism is proved in the same way as Theorem 1 loc.cit. By a specialization argument as in the proof of Theorem 1 in [S2] using Propositions 3 and 4 this time, it is reduced to the case where $k$ is finite. For a finite field $k$, we prove the same formula with $c_{X \bmod D} \in C H^{n}(X \bmod D)$ as in Theorem 1 in [S1] by the same argument using the product formula by Laumon and the Lemmas in Section 2. Then it is enough to show $J_{D, V_{\lambda}}\left(F r_{k}\right)=\tau_{D / F}\left(\rho, \psi_{0}\right)$ where the right hand side is as in loc.cit. It is easily checked by comparing the Definition 10(1) of the left hand side with the definition loc.cit. in terms of Gauss sums. We leave the details to the reader.

(c) Determinant and Jacobi sum for motives. Finally we consider an arithmetic situation. Let $k$ and $F$ be finite extensions of the rational number field $\mathbb{Q}$ and $U$ be a smooth $k$-scheme. We write $M_{\mathbb{Q}, F}^{\prime}(U)=M_{F}(U)$ etc. for short.

Lemma 5.1. Let $X$ be a proper smooth scheme over $k$ including $U$ as the complement of a divisor $D$ with simple normal crossings. Let $\mathcal{M}=((\mathcal{E}, \nabla), V, \rho)$ be an object of $M_{F}(U)$ and assume that the action of the local monodromy $T_{i}$ on $V$ at each component $D_{i}$ of $D$ is quasi-unipotent. Then there exists a unique extension $\left(\mathcal{E}_{X}, \nabla\right)$ of $(\mathcal{E}, \nabla)$ such that the roots $\tau$ of the characteristic polynomials $\Phi_{\mathcal{E}_{X}, i}(T)$ are rational numbers satisfying $0<\tau \leq 1$. Further the $\mathcal{O}_{X}$-module $\mathcal{E}_{X}$ is locally free and, if $(\mathcal{E}, \nabla)$ has a multiplication by $F$, it is extended to $\mathcal{E}_{X}$.

Proof. Since the monodromy is quasi-unipotent, and by $T_{i}=\exp \left(-2 \pi \sqrt{-1} \operatorname{res}_{i} \nabla\right)$ (Chap. II, Theorem 1.7, [D1]), the roots are rational numbers. By Proposition 5.4 loc.cit., there exists a unique extension $\left(\mathcal{E}_{X}, \nabla\right)$ satisfying the condition after extending the scaler to $\mathbb{C}$ and it is locally free. Since the condition is invariant by any automorphism of $\mathbb{C}$ over $k$, it is defined over $k$. By the uniqueness, the multiplication is extended. 
We call the extension $\mathcal{E}_{X}$ defined in the Lemma the canonical extension of $\mathcal{E}$. Let $(\mathcal{E}, \nabla)$ be as in Lemma 5.1 with a multiplication by $F$, and let $\mathcal{E}_{X}$ be the canonical extension. We consider a finite decreasing filtration $F i l$ on $\mathcal{E}_{X}^{(F)}$ by coherent

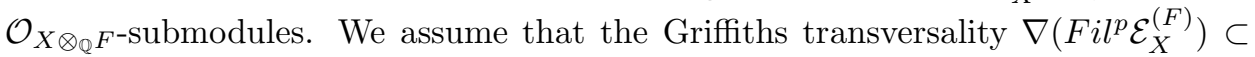
$F i l^{p-1} \mathcal{E}_{X}^{(F)} \otimes_{\mathcal{O}_{X}} \Omega_{X}^{1}(\log D)$ is satisfied. It induces a filtration also denoted by Fil on the de Rham complex $\operatorname{DR}\left(\mathcal{E}_{X}^{(F)}\right)=\left(\mathcal{E}_{X}^{(F)} \otimes \Omega_{X}^{\bullet}(\log D)\right)$ by

$$
\left(F i l^{p} \operatorname{DR}\left(\mathcal{E}_{X}^{(F)}\right)\right)^{q}=\left(F i l^{p-q} \mathcal{E}_{X}^{(F)} \otimes \Omega_{X}^{q}(\log D)\right) .
$$

In the following, we will consider triples $\tilde{\mathcal{M}}=\left(V_{\lambda}, \mathcal{M}, F i l\right)$ as in the definition below.

Definition 12. Let $k$ and $F$ be finite extensions of $\mathbb{Q}, U$ be a smooth variety over $k$ and $\lambda$ be a finite place of $F$. We define a collection $\tilde{\mathcal{M}}_{F, \lambda}(U)$ whose objects are triples $\tilde{\mathcal{M}}=\left(V_{\lambda}, \mathcal{M}, F i l\right)$ consisting of a smooth $F_{\lambda}$-sheaf $V_{\lambda}$ on $U^{\text {et }}$, an object $\mathcal{M}$ of $M_{F}(U)$ and a filtration $F i l$ on the canonical extension $\mathcal{E}_{X}^{(F)}$ satisfying the following finiteness and compatibility conditions:

(1) There exists an $\mathcal{O}_{k}$-scheme $U_{\mathcal{O}_{k}}$ of finite type and a smooth $F_{\lambda}$-sheaf $V_{\ell}$ on $U_{\mathcal{O}_{k}}^{e t}$ such that $U=U_{\mathcal{O}_{k}} \otimes k$ and $V_{\lambda}$ on $U$ is the pull-back of $V_{\lambda}$ on $U_{\mathcal{O}_{k}}$.

(2) The local system $V \otimes F_{\lambda}$ on $U^{a n}$ is isomorphic to the pull-back of $V_{\lambda}$ by $U^{a n} \rightarrow U^{e t}$.

By the assumption (1) and by the monodromy theorem of Grothendieck, the local monodromy of $V_{\lambda}$ along each component of $D$ is quasi-unipotent. By the assumption (2), it is also quasi-unipotent for $V$. Hence by Lemma 5.1, there is a canonical extension $\mathcal{E}_{X}^{(F)}$. If $X=U=\operatorname{Spec} k$ and $\operatorname{rank} \tilde{\mathcal{M}}=1$, a filtration $F i l$ is determined by a function $n: \operatorname{Spec} k \otimes F \rightarrow \mathbb{Z}$ by the condition $\operatorname{Gr}^{p} \mathcal{E}_{X}^{(F)}(x) \neq 0$ if and only if $p=n(x)$ for $x \in \operatorname{Spec} k \otimes F$. By this correspondence, we identify a filtration with an element in $\mathbb{Z}^{\mathrm{Spec} k \otimes F}$. The isomorphism class group of the triples in $\tilde{\mathcal{M}}_{F, \lambda}(U)$ of rank 1 on $\operatorname{Spec} k$ is

$$
\lim _{S} \operatorname{Hom}\left(G_{k, S}^{\mathrm{ab}}, F_{\lambda}^{\times}\right) \times\left((k \otimes F)^{\times} \backslash(k \otimes F \otimes \mathbb{C})^{\times} /\left(F^{\times}\right)^{\operatorname{Hom}(k, \mathbb{C})}\right) \times \mathbb{Z}^{\text {Spec } k \otimes F} .
$$

Here $S$ runs finite sets of places of $k$ and $G_{k, S}$ is the quotient of $G_{k}$ classifying extensions unramified outside $S$.

For $\mathbf{a}=\sum_{a} n_{a}[a] \in B_{F}^{0}(k)$ fixed by $G_{k}$ and $G_{F}$, we define a triple

$$
\tilde{J}(\mathbf{a})=(J(\mathbf{a}),[\Gamma(c \mathbf{a})],\langle\mathbf{a}\rangle) \in \tilde{\mathcal{M}}_{F, \lambda}(U)
$$

of rank 1. For $a \in \mathbb{Q} / \mathbb{Z}$, let $\langle a\rangle \in \mathbb{Q} \cap[0,1)$ denote the fractional part $\langle a\rangle \equiv a \bmod 1$. We define a morphism $\Gamma(c$ ? $) \mathbb{B}=\bigoplus_{\mathbb{Q} / \mathbb{Z}} \mathbb{Z} \rightarrow \mathbb{C}^{\times}$to be the multiplicative map defined by $a \in \mathbb{Q} / \mathbb{Z} \mapsto \Gamma(1-\langle a\rangle)$ and a morphism \langle\rangle$: \mathbb{B} \rightarrow \mathbb{Q}$ to be the linear map induced by \langle\rangle .

Definition 13. For $\mathbf{a}=\sum_{a} n_{a}[a] \in B_{F}^{0}(k)$ fixed by $G_{k}$ and $G_{F}$, we define $\tilde{J}(\mathbf{a}) \in$ $\tilde{\mathcal{M}}_{F, \lambda}(U)$ of rank 1 as a triple $(J(\mathbf{a}),[\Gamma(c \mathbf{a})],\langle\mathbf{a}\rangle)$ consisting of

(1) The $F_{\lambda}$-representation $J(\mathbf{a})$ of $G_{k}^{\text {ab }}$ defined in Definition 10 (3).

(2) The triple $[\Gamma(c \mathbf{a})]=\left(k \otimes F, F, \times \Gamma(c \mathbf{a})^{-1}: k \otimes F \otimes \mathbb{C} \rightarrow F^{H o m(k, \mathbb{C})}\right) \in M_{F}(k)$. Here $\Gamma(c \mathbf{a})=(\Gamma(c((\sigma, \tau)(\mathbf{a}))))_{\sigma, \tau} \in(k \otimes F \otimes \mathbb{C})^{\times}=\left(\mathbb{C}^{\times}\right)^{\operatorname{Hom}(k, \mathbb{C}) \times \operatorname{Hom}(F, \mathbb{C})}$. For $\sigma: k \rightarrow \mathbb{C}$ and $\tau: F \rightarrow \mathbb{C}$, we take isomorphisms $\hat{\mathbb{Z}}(1)_{\bar{k}} \simeq \hat{\mathbb{Z}}(1)_{\mathbb{C}}$ and 
$\hat{\mathbb{Z}}(1)_{\bar{F}} \simeq \hat{\mathbb{Z}}(1)_{\mathbb{C}}$ extending $\sigma$ and $\tau$ and $(\sigma, \tau): \operatorname{Hom}\left(\hat{\mathbb{Z}}(1)_{\bar{k}}, \mathbb{Q} / \mathbb{Z}(1)_{\bar{F}}\right) \rightarrow$ $\operatorname{Hom}\left(\hat{\mathbb{Z}}(1)_{\mathbb{C}}, \mathbb{Q} / \mathbb{Z}(1)_{\mathbb{C}}\right)=\mathbb{Q} / \mathbb{Z}$ denotes the induced isomorphism.

(3) The filtration on $k \otimes F$ such that the index where $G r^{n} \neq 0$ is given by the integers $\langle\mathbf{a}\rangle=\left(\left\langle\rho_{j}(\mathbf{a})\right\rangle\right)_{j} \in \mathbb{Z}^{\mathrm{Spec} k \otimes F}$. For a component $K_{j}$ in $k \otimes F=$ $\prod_{j} K_{j}$, we take isomorphisms $\hat{\mathbb{Z}}(1)_{\bar{k}} \simeq \hat{\mathbb{Z}}(1)_{\bar{K}_{j}}$ and $\hat{\mathbb{Z}}(1)_{\bar{F}} \simeq \hat{\mathbb{Z}}(1)_{\bar{K}_{j}}$ extending the inclusions and $\rho_{j}: \operatorname{Hom}\left(\hat{\mathbb{Z}}(1)_{\bar{k}}, \mathbb{Q} / \mathbb{Z}(1)_{\bar{F}}\right) \rightarrow \operatorname{Hom}\left(\hat{\mathbb{Z}}(1)_{\bar{K}_{j}}, \mathbb{Q} / \mathbb{Z}(1)_{\bar{K}_{j}}\right)=$ $\mathbb{Q} / \mathbb{Z}$ denotes the induced isomorphism.

In (2), since $\mathbf{a}$ is fixed by $G_{k}$ and $G_{F}$, the image $(\sigma, \tau)(\mathbf{a}) \in \mathbb{B}$ is independent of the choice of isomorphisms $\hat{\mathbb{Z}}(1)_{\bar{k}} \simeq \hat{\mathbb{Z}}(1)_{\mathbb{C}}$ and $\hat{\mathbb{Z}}(1)_{\bar{F}} \simeq \hat{\mathbb{Z}}(1)_{\mathbb{C}}$ extending $\sigma$ and $\tau$. Similarly in (3), the image $\rho_{j}(\mathbf{a}) \in \mathbb{B}$ is independent of the choice of isomorphisms $\hat{\mathbb{Z}}(1)_{\bar{k}} \simeq \hat{\mathbb{Z}}(1)_{\bar{K}_{j}}$ and $\hat{\mathbb{Z}}(1)_{\bar{F}} \simeq \hat{\mathbb{Z}}(1)_{\bar{K}_{j}}$ extending the inclusions.

The main result in this section is a combination of Theorem 1 in Section 4 and of Theorem S for a triple $\tilde{\mathcal{M}}=\left(V_{\lambda}, \mathcal{M}, F i l\right) \in \tilde{\mathcal{M}}_{F, \lambda}(U)$.

Definition 14. (1) The determinant of the cohomology $\operatorname{det}_{F} R \Gamma_{c}(U, \tilde{\mathcal{M}}) \in$ $\tilde{\mathcal{M}}_{F, \lambda}(k)$ is the triple $\left(\operatorname{det}_{F_{\lambda}} R \Gamma_{c}\left(U_{\bar{k}}, V_{\lambda}\right), \operatorname{det}_{F} R \Gamma_{c}(U, \mathcal{M}), F i l\right)$ described as follows. The first two components are defined in Sections 5(b) and 4(f) respectively. The filtration $F i l$ on $\operatorname{det}_{F} R \Gamma_{c}(U, D R(\mathcal{E}))$ is induced by the filtration on the de Rham complex $D R\left(\mathcal{E}_{X}^{(F)}\right)$ by the isomorphism $R \Gamma_{c}(U, D R(\mathcal{E})) \simeq$ $R \Gamma\left(X^{(F)}, D R\left(\mathcal{E}_{X}^{(F)}\right)\right)$ of perfect complexes of $k \otimes F$-modules.

(2) For an element $c \in C H^{n}(X \bmod D)$, the pairing $\left(\operatorname{det}_{F} \tilde{\mathcal{M}}, c\right) \in \tilde{\mathcal{M}}_{F, \lambda}(U)$ is the triple $\left(\left(\operatorname{det}_{F_{\lambda}} V_{\lambda}, c\right),\left(\operatorname{det}_{F} \mathcal{M}, c\right), F i l\right)$ as follows. The first two components are defined in Sections 3(a) and 5(a) respectively. The filtration is defined by the function $n: \operatorname{Spec} k \otimes F \rightarrow \mathbb{Z}, n(y)=\sum_{X_{j} \subset f^{*}(y)} n_{j} \operatorname{deg}_{j}\left(\left.c\right|_{X_{j}}\right)$. Here, $f$ denotes the map $X^{(F)} \rightarrow$ Spec $k \otimes F$ and for a connected component $X_{j}$ of $X^{(F)}, n_{j}$ denotes the unique integer such that $G r^{n_{j}}\left(\operatorname{det} \mathcal{E}_{X}^{(F)}\right) \neq 0$ at the generic point of $X_{j}$ and $\operatorname{deg}_{j}$ denotes the map $C H^{n}\left(X_{j}\right) \rightarrow C H^{0}\left(f\left(X_{j}\right)\right)=\mathbb{Z}$.

(3) The Jacobi sum $J_{D, \tilde{\mathcal{M}}} \in \tilde{\mathcal{M}}_{F, \lambda}(k)$ is the triple $\tilde{J}\left(\mathbf{a}_{D, \tilde{\mathcal{M}}}\right)$ for $\mathbf{a}_{D, \tilde{\mathcal{M}}}=\mathbf{a}_{D, V_{\lambda}} \in$ $B_{F}^{0}(k)$ where $\mathbf{a}_{D, V_{\lambda}} \in B_{F_{\lambda}}^{0}(k)^{G_{k}}$ is the element defined for the $F_{\lambda}$-sheaf $V_{\lambda}$ (Definition 13).

By the condition (2) in Definition 12, $\mathbf{a}_{D, V_{\lambda}} \in B_{F_{\lambda}}^{0}(k)^{G_{k}}$ comes from an element $\mathbf{a}_{D, \tilde{\mathcal{M}}} \in B_{F}^{0}(k)$ fixed also by $G_{F}$.

We define the notion that a triple $\tilde{\mathcal{M}}$ is determinantally motivic. If $U=\operatorname{Spec} k$ and $\operatorname{rank} \tilde{\mathcal{M}}=1$, a triple is called motivic if it comes from an algebraic Hecke character. In general, it is called motivic if the determinant $\operatorname{det} \tilde{\mathcal{M}}$ is motivic at each point $x \in U$.

Definition 15. Let $k$ and $F$ be finite extensions of $\mathbb{Q}$.

(1) A triple $\tilde{\mathcal{M}}=\left(V_{\lambda}, \mathcal{M}\right.$, Fil $)$ of rank 1 on $U=\operatorname{Spec} k$ and is called motivic if there exists an algebraic Hecke character $\chi$ of $k$ with value in $F$ such that the following conditions (1a)-(1c) are satisfied.

(1a) For a finite place $p$ of $k$ prime to the conductor of $\chi$ and to the characteristic of $\lambda$, the $\lambda$-adic representation $V_{\lambda}$ of the absolute Galois group $G_{k}$ of $k$ is unramified and the action of the geometric Frobenius $F r_{p}$ on $V_{\lambda}$ is the multiplication by $\chi(p)$. 
(1b) The class $[\mathcal{M}] \in M P i c_{F}(k)=(k \otimes F)^{\times} \backslash(k \otimes F \otimes \mathbb{C})^{\times} /\left(F^{\times}\right)^{\operatorname{Hom}(k, \mathbb{C})}$ is equal to the period $p^{\prime}(\chi)$ ([D4], 8.7) of a motive $M(\chi)$ for $\chi$ in the category of motives generated by potentially $\mathrm{CM}$ abelian varieties.

(1c) If we decompose $k \otimes F \simeq \prod_{i} K_{i}$ into products of fields and let $\chi_{a l g}=$ $\prod_{i} N_{K_{i} / F}^{n_{i}} \circ$ can, then $\operatorname{Gr}^{p}\left(\mathcal{E}_{X}^{(F)} \otimes_{k \otimes F} K_{i}\right) \neq 0$ if and only if $p=n_{i}$.

(2) A triple $\tilde{\mathcal{M}}=\left(V_{\lambda}, \mathcal{M}, F i l\right)$ on a smooth variety $U$ over $k$ is said to be determinantally motivic if for any closed point $x \in U$, the fiber at $x$ of the determinant $\operatorname{det}_{F} \tilde{\mathcal{M}}=\left(\operatorname{det}_{F_{\lambda}} V_{\lambda}\right.$, $\left.\operatorname{det}_{F} \mathcal{M}, F i l\right)$ is motivic.

Theorem 2. Let $k$ and $F$ be number fields and $U$ be the complement of a normal crossing divisor $D$ in a projective smooth scheme $X$ over $k$. Let $\tilde{\mathcal{M}}=\left(V_{\lambda}, \mathcal{M}, F i l\right)$ be a triple of a smooth $F_{\lambda}$-sheaf $V_{\lambda}$ on $U^{\text {et }}$, an object $\mathcal{M}$ of $M_{F}(U)$ of rank $r$ satisfying conditions (1) and (2) and a filtration Fil on the canonical extension $\mathcal{E}_{X}^{(F)}$. Assume that the spectral sequence $E_{1}^{p, q}=H^{p+q}\left(X^{(F)}, G r^{p} D R\left(\mathcal{E}_{X}^{(F)}\right)\right) \Rightarrow$ $H^{p+q}\left(X^{(F)}, D R\left(\mathcal{E}_{X}^{(F)}\right)\right)$ degenerates at $E_{1}$-terms and that the Gersten conjecture holds for discrete valuation rings which are local rings of a smooth model of $X$ over some open subscheme of $\mathcal{O}_{k}$. Then

(1) There is an isomorphism

$$
\operatorname{det}_{F} R \Gamma_{c}(U, \tilde{\mathcal{M}}) \otimes\left(\operatorname{det}_{F} R \Gamma_{c}(U, 1)\right)^{\otimes-r} \simeq\left(\operatorname{det}_{F} \tilde{\mathcal{M}}, c_{X \bmod D}\right) \otimes J_{D, \tilde{\mathcal{M}}}^{\otimes-1} .
$$

(2) Further if $\tilde{\mathcal{M}}$ is determinantally motivic, then $\operatorname{det}_{F} R \Gamma_{c}(U, \tilde{\mathcal{M}})$ is also motivic.

Proof. (1) The $F_{\lambda}$-sheaves in the first component are isomorphic to each other by Theorem S. By Theorem $1^{\prime}$ in Section 4, to prove the second components, the objects of $P_{F}(k)$, are isomorphic, it is sufficient to show that

$$
\Gamma\left(\nabla: \mathcal{E}_{X}\right)=\Gamma\left(c \mathbf{a}_{D, \tilde{\mathcal{M}}}\right)
$$

$\in(k \otimes F \otimes \mathbb{C})^{\times}$. Let $n_{c}(U, \tilde{\mathcal{M}}), n\left(\operatorname{det} \tilde{\mathcal{M}}, c_{X \bmod D}\right)$ etc. be the element $\mathbb{Z}^{\text {Spec } k \otimes F}$ corresponding to the filtration on $\operatorname{det}_{F} R \Gamma\left(X, D R\left(\mathcal{E}_{X}\right)\right)$, $\left(\operatorname{det} \mathcal{E}, c_{X \bmod D}\right)$ etc. To prove the filtration are the same, it is enough to show

$$
n_{c}(U, \tilde{\mathcal{M}})-r n_{c}(U, 1)=n\left(\operatorname{det} \tilde{\mathcal{M}}, c_{X \bmod D}\right)-\left\langle\mathbf{a}_{D, \tilde{\mathcal{M}}}\right\rangle
$$

in $\mathbb{Z}^{\mathrm{Spec} k \otimes F}$

Let $\mathbf{a}_{i}=\sum_{a} n_{i, a}[a] \in B_{k_{i}}(F)$ be the element which appears in the definition of $\mathbf{a}_{D, V_{\lambda}}$. By $T_{i}=\exp \left(-2 \pi \sqrt{-1} \cdot \operatorname{res}_{i} \nabla\right)$ and since the eigenvalues of $\operatorname{res}_{i} \nabla$ are in $(0,1]$, the image of $\Phi_{\mathcal{E}_{X}, i}(T) \in k_{i}(T)^{\times}$in $\left(k_{i} \otimes F \otimes \mathbb{C}(T)\right)^{\times}=\left(\mathbb{C}(T)^{\times}\right)^{\operatorname{Hom}\left(k_{i}, \mathbb{C}\right) \times \operatorname{Hom}(F, \mathbb{C})}$ is equal to $\left(\prod_{a}(T-(1-\langle(\sigma, \tau)(a)\rangle))^{n_{i, a}}\right)_{(\sigma, \tau)}$. By $\Phi_{\mathcal{E}_{X}}(T)=\prod_{i} N_{k_{i} / k} \Phi_{\mathcal{E}_{X}, i}(T)^{c_{i}}$ and $\mathbf{a}_{D, \tilde{\mathcal{M}}}=\sum_{i} c_{i} \operatorname{Tr}_{k_{i} / k} \mathbf{a}_{i}$, the image of $\Phi_{\mathcal{E}_{X}}(T) \in k(T)^{\times}$in $(k \otimes F \otimes \mathbb{C}(T))^{\times}=$ $\left(\mathbb{C}(T)^{\times}\right)^{\operatorname{Hom}(k, \mathbb{C}) \times \operatorname{Hom}(F, \mathbb{C})}$ is equal to $\left(\prod_{a}(T-(1-\langle(\sigma, \tau)(a)\rangle))^{n_{a}}\right)_{(\sigma, \tau)}$ for $\mathbf{a}_{D, \tilde{\mathcal{M}}}=$ $\sum_{a} n_{a}[a]$. From this the equality $\Gamma\left(\nabla: \mathcal{E}_{X}\right)=\Gamma\left(c \mathbf{a}_{D, \tilde{\mathcal{M}}}\right)$ follows immediately. Also we have $\operatorname{Tr} \operatorname{res}_{i} \nabla=\left(r-\left\langle(\sigma, \tau)\left(\mathbf{a}_{i}\right)\right\rangle\right)_{(\sigma, \tau)}$ in $k_{i}^{\times} \subset\left(k_{i} \otimes F \otimes \mathbb{C}\right)^{\times}$. Hence we have $-\left\langle\mathbf{a}_{D, \tilde{\mathcal{M}}}\right\rangle=\sum_{i} c_{i} \cdot \operatorname{Tr}_{k_{i} / k}\left(\operatorname{Tr} \operatorname{res}_{i} \nabla-r\right)$ in $\mathbb{Z}^{\operatorname{Spec} k \otimes F} \subset k \otimes F$. Therefore the second equality becomes

$$
n_{c}(U, \tilde{\mathcal{M}})-r n_{c}(U, 1)=n\left(\operatorname{det} \tilde{\mathcal{M}}, c_{X \bmod D}\right)+\sum_{i} c_{i} \cdot \operatorname{Tr}_{k_{i} / k}\left(\operatorname{Tr} \operatorname{res}_{i} \nabla-r\right) .
$$

Proof of this equality will complete the proof of (1).

We compute $n_{c}(U, \tilde{\mathcal{M}})$. To simplify the notation, we write dim for the rank of the $k \otimes F$-module, which is a function on Spec $k \otimes F$ and write $\chi$ for the alternating 
sum of dim. By the assumption that the spectral sequence degenerates at $E_{1}$ and by the definition of the filtration on $\operatorname{det}_{F} R \Gamma\left(X^{(F)}, D R\left(\mathcal{E}_{X}^{(F)}\right)\right)$, we have $n_{c}(U, \tilde{\mathcal{M}})=$ $\sum_{p} p \cdot \chi\left(X^{(F)}, G r^{p} D R\left(\mathcal{E}_{X}^{(F)}\right)\right)$. Since $\left(G r^{p} D R\left(\mathcal{E}_{X}^{(F)}\right)\right)^{q}=G r^{p-q} \mathcal{E}_{X}^{(F)} \otimes \Omega_{X}^{q}(\log D)$, by putting $r=p-q$, we have

$$
\begin{aligned}
n_{c}(U, \tilde{\mathcal{M}}) & =\sum_{q, r}(-1)^{q}(q+r) \chi\left(X^{(F)}, G r^{r} \mathcal{E}_{X}^{(F)} \otimes \Omega_{X}^{q}(\log D)\right) \\
=\sum_{r} & \left(r \sum_{q}(-1)^{q} \chi\left(X^{(F)}, G r^{r} \mathcal{E}_{X}^{(F)} \otimes \Omega_{X}^{q}(\log D)\right)\right) \\
& +\sum_{q}(-1)^{q} q \chi\left(X^{(F)}, \mathcal{E}_{X}^{(F)} \otimes \Omega_{X}^{q}(\log D)\right) .
\end{aligned}
$$

We write $n_{c}^{1}(U, \tilde{\mathcal{M}})$ and $n_{c}^{2}(U, \tilde{\mathcal{M}})$ for the first term and the second term respectively. We prove the equalities

$$
\begin{gathered}
n_{c}^{1}(U, \tilde{\mathcal{M}})=n\left(\operatorname{det}_{F} \tilde{\mathcal{M}}, c_{X \bmod D}\right), \\
n_{c}^{2}(U, \tilde{\mathcal{M}})-r n_{c}^{2}(U, 1)=\sum_{i} c_{i} \cdot \operatorname{Tr}_{k_{i} / k}\left(\operatorname{res}_{i} \nabla-r\right) .
\end{gathered}
$$

Since $n_{c}^{1}(U, 1)=0$, the equalities prove the assertion (1) of Theorem 2. We prove the equality (I). For a connected component $X_{j}$ of $X^{(F)}$, let $n_{j}^{r}$ be the rank of $G r^{r}$ at the generic point and put $c_{j}=\operatorname{deg}_{j}\left(\left.c_{X \bmod D}\right|_{X_{j}}\right)$ where $\operatorname{deg}_{j}: C H^{n}\left(X_{j}\right) \rightarrow$ $C H^{n}(y)=\mathbb{Z}$ for $y=f\left(X_{j}\right) \in \operatorname{Spec} k \otimes F$. By Riemann-Roch, we have

$$
\sum_{q}(-1)^{q} \chi_{\kappa(y)}\left(X_{j},\left.G r^{r} \mathcal{E}_{X}^{(F)} \otimes \Omega_{X}^{q}(\log D)\right|_{X_{j}}\right)=n_{j}^{r} \cdot c_{j} .
$$

Since the integer $n_{j}$ appearing in the definition of $n\left(\operatorname{det}_{F} \tilde{\mathcal{M}}, c_{X \bmod D}\right)$ is $n_{j}=$ $\sum_{r} r n_{j}^{r}$, the function $n_{c}^{1}(U, \tilde{\mathcal{M}}): \operatorname{Spec} k \otimes F \rightarrow \mathbb{Z}$ is $y \mapsto \sum_{X_{j} \subset f^{*}(y)} n_{j} c_{j}$. It is $n\left(\operatorname{det}_{F} \tilde{\mathcal{M}}, c_{X \bmod D}\right)$ by definition.

To prove (II), we show

Lemma 5.2. Let $X$ be a proper smooth scheme over a field $k$ of dimension $n$ and $\mathcal{F}$ be a locally free $\mathcal{O}_{X}$-module of rank $n$. Let $\mathcal{E}_{1}$ and $\mathcal{E}_{2}$ be coherent $\mathcal{O}_{X}$-modules whose ranks are the same on a dense open subscheme of $X$. Then

$$
\begin{gathered}
\sum_{q}(-1)^{q} q \chi\left(X, \mathcal{E}_{1} \otimes \wedge^{q} \mathcal{F}\right)-\sum_{q}(-1)^{q} q \chi\left(X, \mathcal{E}_{2} \otimes \wedge^{q} \mathcal{F}\right) \\
=(-1)^{n}\left(c_{1}\left(\mathcal{E}_{1}\right)-c_{1}\left(\mathcal{E}_{2}\right), c_{n-1}(\mathcal{F})\right) .
\end{gathered}
$$

Proof. By Riemann-Roch, the left hand side is the degree 0 part of

$$
\left(\operatorname{ch}\left(\mathcal{E}_{1}\right)-\operatorname{ch}\left(\mathcal{E}_{2}\right)\right) \cdot\left(\sum_{q}(-1)^{q} q \cdot \operatorname{ch}\left(\wedge^{q} \mathcal{F}\right)\right) \cdot t d\left(\Omega_{X}^{1}\right)
$$

Since $\operatorname{ch}\left(\mathcal{E}_{1}\right)-\operatorname{ch}\left(\mathcal{E}_{2}\right)$ is $c_{1}\left(\mathcal{E}_{1}\right)-c_{1}\left(\mathcal{E}_{2}\right)+$ higher terms, it is sufficient to show that $\sum_{q}(-1)^{q} q \cdot \operatorname{ch}\left(\wedge^{q} \mathcal{F}\right)$ is $(-1)^{n} c_{n-1}(\mathcal{F})+$ higher terms. By the splitting principle, we put $\sum c_{i}(\mathcal{F}) T^{n-i}=\prod_{i=1}^{n}\left(T+\alpha_{i}\right)$. If we put $a_{i}=\exp \alpha_{i}$, the Chern character $\operatorname{ch}\left(\wedge^{q} \mathcal{F}\right)$ is the $q$-th fundamental symmetric polynomial $s_{q}$ of $a_{i}$. We consider a polynomial $f(T)=\sum(-1)^{q} s_{q} T^{n-q}=\prod\left(T-a_{i}\right)$ and compute the derivative $f^{\prime}(1)$ in two ways. First we have $f^{\prime}(1)=\sum(-1)^{q}(n-q) s_{q}=n \sum(-1)^{q} s_{q}-\sum(-1)^{q} q s_{q}$. Since $\sum(-1)^{q} s_{q}=f(1)=(-1)^{n} c_{n}(\mathcal{F})+$ higher terms, we have $\sum(-1)^{q} q s_{q}=$ 
$-f^{\prime}(1)$ modulo codimension $n$. On the other hand $f^{\prime}(1)=\sum_{i} \prod_{j \neq i}\left(1-a_{j}\right)=$ $(-1)^{n-1} c_{n-1}(\mathcal{F})+$ higher terms. Hence we have $\sum(-1)^{q} q s_{q}=(-1)^{n} c_{n-1}(\mathcal{F})+$ higher terms and the Lemma is proved.

By the Lemma applied to $\mathcal{E}_{1}=\mathcal{E}_{X}^{(F)}, \mathcal{E}_{2}=\mathcal{O}_{X}(-D)^{(F)}$ and $\mathcal{F}=\Omega_{X}^{1}(\log D)$, we have

$$
\begin{aligned}
n_{c}^{2}(U, \tilde{\mathcal{M}})-r n_{c}^{2}(U, 1)= & -\left(c_{1}\left(\mathcal{E}_{X}^{(F)}\right)-r c_{1}\left(\mathcal{O}_{X}(-D)^{(F)}\right)\right) \\
& \cdot(-1)^{n-1} c_{n-1}\left(\Omega_{X}^{1}(\log D)\right) .
\end{aligned}
$$

Therefore to complete the proof it is enough to show

$$
\left(-c_{1}\left(\mathcal{E}_{X}^{(F)}\right)\right) \cdot(-1)^{n-1} c_{n-1}\left(\Omega_{X}^{1}(\log D)\right)=\sum_{i} c_{i} \cdot \operatorname{Tr}_{k_{i} / k} \operatorname{Tr} \operatorname{res}_{i} \nabla
$$

in $\mathbb{Z}^{\text {Spec } k \otimes F} \subset k \otimes F$. We compute $c_{1}\left(\mathcal{E}_{X}^{(F)}\right)$ by using the residue.

Lemma 5.3. Let $X$ be a proper smooth scheme over a field $k$ of characteristic 0 and $U$ be the complement of a divisor $D$ with simple normal crossings. Let $\nabla: \mathcal{E}_{X} \rightarrow$ $\mathcal{E}_{X} \otimes \Omega_{X}^{1}(\log D)$ be a logarithmic integrable connection. Let $k_{i}$ be the constant field of an irreducible component $D_{i}$ of $D$ and $\operatorname{Tr} \operatorname{res}_{i} \nabla \in k_{i}$. Let $\partial: \bigoplus_{i} k_{i} \rightarrow H^{1}\left(X, \Omega_{X}^{1}\right)$ be the boundary map of the exact sequence $0 \rightarrow \Omega_{X}^{1} \rightarrow \Omega_{X}^{1}(\log D) \rightarrow \bigoplus_{i} \mathcal{O}_{D_{i}} \rightarrow 0$. Then we have

$$
c_{1}\left(\mathcal{E}_{X}\right)=-\partial\left(\operatorname{Tr} \operatorname{res}_{i} \nabla\right)_{i} .
$$

Proof. The first Chern class $c_{1}\left(\mathcal{E}_{X}\right)$ is the class of the push of the $\mathbb{G}_{m}$-torsor $\operatorname{det} \mathcal{E}_{X}$ by $d \log : \mathbb{G}_{m} \rightarrow \Omega_{X}^{1}$. The $\Omega_{X}^{1}$-torsor is trivialized locally by $\nabla \log e$ for a local basis $e$ of $\operatorname{det} \mathcal{E}_{X}$. Hence it is the inverse image of $\sum_{i} \operatorname{Tr} \operatorname{res}_{i} \nabla \in \bigoplus_{i} \mathcal{O}_{D_{i}}$ by $\Omega_{X}^{1}(\log D) \rightarrow \bigoplus_{i} \mathcal{O}_{D_{i}}$. Since the boundary map is the minus of the class of torsor, the Lemma is proved.

By Lemma 5.3, we have

$$
\begin{aligned}
& \left(-c_{1}\left(\mathcal{E}_{X}^{(F)}\right)\right) \cdot(-1)^{n-1} c_{n-1}\left(\Omega_{X}^{1}(\log D)\right) \\
= & \sum_{i} \operatorname{Tr}_{k_{i} / k}\left(\operatorname{res}_{i} \nabla \cdot \operatorname{deg}_{k_{i}}\left((-1)^{n-1} c_{n-1}\left(\left.\Omega_{X}^{1}(\log D)\right|_{D_{i}}\right)\right)\right) \\
= & \sum_{i} c_{i} \cdot \operatorname{Tr}_{k_{i} / k} \operatorname{Tr} \operatorname{res}_{i} \nabla .
\end{aligned}
$$

Thus the proof of Theorem 2 (1) is now complete.

(2) By the Corollary of Proposition 1 in Section 2 and by the definition of determinantally motivic, $\left(\operatorname{det}_{F} \tilde{\mathcal{M}}, c\right)$ is motivic for $c \in C H^{n}(X \bmod D)$. Hence it is sufficient to prove

Lemma 5.4. For $\mathbf{a} \in \mathbb{B}^{0}$, the triple $\tilde{J}(\mathbf{a})$ over the abelian number field $k=F=F_{\mathbf{a}}$ is motivic.

Proof. We may assume the coefficient $n_{a}$ of $\mathbf{a}=\sum_{a} n_{a}[a]$ is positive, $n_{a} \geq 0$, for all $a \in \mathbb{Q} / \mathbb{Z}$. In fact we put $\mathbf{a}^{+}=\sum_{n_{a}>0} n_{a}[a]+\left[-\sum_{n_{a}>0} n_{a} a\right]$ and $\mathbf{a}^{-}=$ $\sum_{n_{a}<0}\left(-n_{a}\right)[a]+\left[\sum_{n_{a}<0} n_{a} a\right] \in \mathbb{B}^{0}$. Then $\mathbf{a}^{+}$and $\mathbf{a}^{-}$satisfy the condition $n_{a} \geq 0$, fixed by $G_{\mathbf{a}}$ and $\mathbf{a}=\mathbf{a}^{+}-\mathbf{a}^{-}$. Since the algebraic Hecke character $J(\mathbf{a})$ is the quotient $J\left(\mathbf{a}^{+}\right) J\left(\mathbf{a}^{-}\right)^{-1}$, we may assume $n_{a} \geq 0$. Further since $J(\mathbf{a})=J\left(\mathbf{a}_{0}\right)$ for $\mathbf{a}_{0}=\mathbf{a}-n_{0}[0]$, we may assume $n_{0}=0$. 
Let $\mathbf{a}=\sum_{i=0}^{n+1}\left[a_{i}\right]$. Clearly we may assume $n \geq 0$. First we consider the case $n=0$. Let $\mathbf{a}=[a / m]+[-a / m]$ for integers $(a, m)=1$. The field $F=$ $F_{\mathbf{a}}$ is then $\mathbb{Q}\left(\cos \frac{2 \pi}{m}\right)$. We compute $\tilde{J}(\mathbf{a})$. For even $m$, let $\epsilon$ be the character of order 2 corresponding to the quadratic extension $\mathbb{Q}\left(\cos \frac{\pi}{m}\right)$ of $F$ and $\tilde{\epsilon}$ be the triple $\left(\epsilon,\left(\tau \circ \sigma^{-1}\left(\exp \frac{a \pi \sqrt{-1}}{m}\right)\right)_{\sigma, \tau}, 0\right)$. For odd $m$, we put $\tilde{\epsilon}=\mathbf{1}$. We show $\tilde{J}(\mathbf{a})=\tilde{\epsilon} \otimes \mathbf{1}(-1)$ and this proves the Lemma for $n=0$. First we compute the algebraic Hecke character $J(\mathbf{a})$. Let $q$ be a finite place of $F=F_{\mathbf{a}}$ prime to $2 m$. The norm $N q$ satisfies $N q= \pm 1 \bmod m$ since $F r_{q}$ fixes a. If $N q \equiv 1 \bmod m$, we have $J(\mathbf{a})(q)=$ $\tau(\chi, \psi) \tau\left(\chi^{-1}, \psi\right)=\chi(-1) N q$ for the $m$-th power residue symbol $\chi=\left(\begin{array}{c}q_{m}^{a}: \kappa(q)^{\times} \\ \mu_{m}\end{array}\right)$. Further

$$
\chi(-1)=(-1)^{\frac{N q-1}{m} a}= \begin{cases}1 & \text { if } m \text { is odd or if } m \text { is even and } N q \equiv 1 \bmod 2 m, \\ -1 & \text { if } m \text { is even and } N q \equiv 1+m \bmod 2 m .\end{cases}
$$

If $N q \equiv-1 \bmod m$, we have $J(\mathbf{a})(q)=\tau_{E_{2}}\left(\chi, \psi \circ \operatorname{Tr}_{E_{2} / \kappa(q)}\right)$ where $E_{2}$ is the quadratic extension of $\kappa(q)$ and $\chi=\left(E_{2}^{\times}\right) \mu_{m}$. Using $\left.\chi\right|_{\kappa(q) \times}=1$, an elementary computation yields $J(\mathbf{a})(q)=(-1)^{\frac{N q+1}{m} a} N q$. Hence we have

$$
J(\mathbf{a})(q)= \begin{cases}N q & \text { if } m \text { is odd or if } m \text { is even and } N q \equiv-1 \bmod 2 m, \\ -N q & \text { if } m \text { is even and } N q \equiv-1+m \bmod 2 m\end{cases}
$$

Thus $J(\mathbf{a})=\epsilon \cdot N$, where $N$ is the norm, is proved. By $\Gamma(s) \Gamma(1-s)=2 \pi \sqrt{-1}$. $\exp (\pi \sqrt{-1} \cdot s) /(\exp (2 \pi \sqrt{-1} \cdot s)-1)$, we have

$$
\Gamma(c \mathbf{a})=2 \pi \sqrt{-1} \times \begin{cases}1 & \text { if } m \text { is odd } \\ \left(\tau \circ \sigma^{-1}\left(\exp \frac{a \pi \sqrt{-1}}{m}\right)\right)_{\sigma, \tau} & \text { if } m \text { is even }\end{cases}
$$

in $(F \otimes F)^{\times} \backslash(F \otimes F \otimes \mathbb{C})^{\times}$. Finally it is clear that $\langle\mathbf{a}\rangle=1$. Thus we have proved $\tilde{J}(\mathbf{a})=\tilde{\epsilon} \otimes \mathbf{1}(-1)$.

Assume $n \geq 1$. We show $\tilde{J}(\mathbf{a})$ appears in the cohomology of a Fermat hypersurface and hence is in the category of motives generated by CM abelian varieties. Let $m$ be an integer satisfying $m a_{i}=0$ for $0 \leq i \leq n+1$. Let $U$ be the complement in $X=\operatorname{Proj} F\left[T_{0}, \cdots, T_{n+1}\right] /\left(\sum_{i} T_{i}\right) \simeq \mathbb{P}_{F}^{n}$ of the divisor $D=\bigcup_{i} D_{i}$ with simple normal crossings, where $D_{i}=\left(T_{i}=0\right)$. Let $X_{m}^{n}$ be the Fermat hypersurface Proj $F\left[T_{0}, \cdots, T_{n}\right] /\left(\sum_{i} T_{i}^{m}\right)$, and $\pi: X_{m}^{n} \rightarrow X$ be the covering $T_{i} \mapsto T_{i}^{m}$ etale over $U$. Over the field $\mathbb{Q}\left(\zeta_{m}\right)$, it is a Galois covering with the Galois group $G=\mu_{m}^{n+1} / \Delta\left(\mu_{m}\right)$. The element a defines a character a : $G \rightarrow \mathbb{Q}\left(\mu_{m}\right)^{\times}$ by $\left(\zeta_{i}\right)_{i} \mapsto \prod_{i} \zeta_{i}^{m\left\langle a_{i}\right\rangle}$. Let $p_{\mathbf{a}}=\sum_{\zeta \in G} \mathbf{a}(\zeta)^{-1} \zeta \in \mathbb{Q}\left(\mu_{m}\right)[G]$ be the projector. Since $\mathbf{a}$ is fixed by $G_{F_{\mathbf{a}}}$, it is in $F_{\mathbf{a}}[G]$. Further the algebraic correspondence $\left[p_{\mathbf{a}}\right]=\sum_{\zeta \in G} \mathbf{a}(\zeta)^{-1}\left[\Gamma_{\zeta}\right]$ where $\left[\Gamma_{\zeta}\right]$ is the graph of the automorphism $\zeta \in G$ on $X_{m, \mathbb{Q}\left(\mu_{m}\right)}^{n}$ is stable by $G_{F_{\mathbf{a}}}$ and is defined over $F_{\mathbf{a}}$. Let $\tilde{\mathcal{M}}_{\mathbf{a}}$ be the $p_{\mathbf{a}}$-component of the direct image $\pi_{*} 1$ on $U$ with the trivial filtration $G r^{0}=\tilde{\mathcal{M}}_{\mathbf{a}}$. We apply Theorem 2 (1) to $\tilde{\mathcal{M}}_{\mathbf{a}}$ on $U$ and show that

$$
H_{c}^{n}\left(U, \tilde{\mathcal{M}}_{\mathbf{a}}\right)(-1) \simeq \tilde{J}(\mathbf{a})
$$

Since the left hand side is in the category generated by CM abelian varieties, this completes the proof of (2). 
It is well-known that $H_{c}^{q}\left(U, \tilde{\mathcal{M}}_{\mathbf{a}}\right)=0$ for $q \neq n$ and $H_{c}^{n}\left(U, \tilde{\mathcal{M}}_{\mathbf{a}}\right)$ is of rank 1 . Also it is easy to check that $\operatorname{det}_{F} R \Gamma_{c}(U, \mathbf{1}) \simeq \mathbf{1}\left((-1)^{n+1}\right)$. Hence the left hand side of Theorem 2 (1) for $\tilde{\mathcal{M}}_{\mathbf{a}}$ on $U$ is $\left(H_{c}^{n}\left(U, \tilde{\mathcal{M}}_{\mathbf{a}}\right)(-1)\right)^{(-1)^{n}}$. We compute the right hand side. The rank of $\tilde{\mathcal{M}}_{\mathbf{a}}$ over $F_{\mathbf{a}}$ is 1 . We compute $c_{X \bmod D}$. The exact sequence

$$
H^{n-1}\left(X, \mathcal{K}_{n}\right) \rightarrow \bigoplus_{i} H^{n-1}\left(D_{i}, \mathcal{K}_{n}\right) \rightarrow C H^{n}(X \bmod D) \rightarrow H^{n}\left(X, \mathcal{K}_{n}\right) \rightarrow 0
$$

is

$$
F^{\times} \rightarrow \bigoplus_{i} F^{\times} \rightarrow C H^{n}(X \bmod D) \rightarrow \mathbb{Z} \rightarrow 0
$$

and a section $\mathbb{Z} \rightarrow C H^{n}(X \bmod D): 1 \mapsto[x]$ is defined for an $F$-rational point $x=\left(t_{0}: \cdots: t_{n+1}\right) \in U(F)$. We show that $c_{n}\left(\Omega_{X}^{1}(\log D)\right.$, res $)=[x]+\left(t_{i}\right)_{i}$. Let $\omega=\sum_{i=1}^{n+1} t_{i} d \log \left(T_{i} / T_{0}\right)$. The zero locus of $\omega$ is $[x]$ and $\operatorname{res}_{D_{i}} \omega=t_{i}$ for $0 \leq i \leq n+1$. Using Lemma 2.1, we have

$$
c_{n}\left(\Omega_{X}^{1}(\log D), \text { res }\right)=[x]+\sum_{i}\left\{t_{i}\right\} \cdot c_{n-1}\left(\Omega_{D_{i}}\left(\left.\log D\right|_{D_{i}}\right)\right)=[x]+\left(t_{i}\right)_{i} .
$$

From this, by an elementary computation, we have $\left(\tilde{\mathcal{M}}_{\mathbf{a}}, c_{X \bmod D}\right)=1$. We also see that the formation of $c_{X \bmod D}$ commutes with base change and we do not need to assume the Gersten resolution in the proof of (1) for this case. The assumption that the spectral sequence degenerates at $E_{1}$ is satisfied by the degeneracy of the Hodge

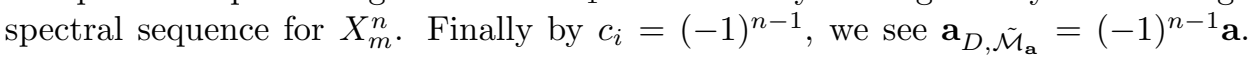
Therefore the right hand side of $(1)$ is $\tilde{J}(\mathbf{a})^{(-1)^{n}}$. Thus $H_{c}^{n}\left(U, \tilde{\mathcal{M}}_{\mathbf{a}}\right)(-1) \simeq \tilde{J}(\mathbf{a})$ is proved and the proof of Theorem 2 is completed.

\section{REFinEMENT FOR CURVES}

In this section, we prove a stronger version, Theorem 3 , of the main result, Theorem 1, for curves. It gives a canonical isomorphism in terms of Deligne's RiemannRoch while Theorem 1 means only the existence of an isomorphism. Throughout in this section, we keep the following notation as in Section 4 . Let $k_{0}, F$ be subfields of $\mathbb{C}$ and $k$ be a finite extension of $k_{0}$. Let $U$ be a smooth curve, $X \supset U$ be a smooth proper curve over $k$ and the reduced divisor $D=X-U$ be the complement.

(a) Preliminaries. For an object $\mathcal{M} \in M_{k_{0}, F}(U)$, we define objects

$$
\operatorname{det}^{\Gamma} R \Gamma_{c}(U, \mathcal{M}), \quad\left(\operatorname{det} \mathcal{M}, \mathbf{c}_{X \bmod D}\right) \in M_{k_{0}, F}(k)
$$

of rank 1, whose isomorphism classes $\operatorname{are~}_{\operatorname{per}}^{\Gamma}(\mathcal{M}),\left(\operatorname{det} \mathcal{M}, c_{X \bmod D}\right) \in M P i c_{k_{0}, F}(k)$ respectively and a canonical isomorphism

$$
\operatorname{det}^{\Gamma} R \Gamma_{c}(U, D R(\mathcal{E})) \otimes \operatorname{det}^{\Gamma} R \Gamma_{c}\left(U, D R\left(\mathcal{O}_{U}\right)\right)^{\otimes-r} \simeq\left(\operatorname{det} \mathcal{E}, \mathbf{c}_{X \bmod D}\right)
$$

of the de Rham components as follows.

Definition 16. Let $\mathcal{M}=((\mathcal{E}, \nabla), V, \rho)$ be an object of $M_{k_{0}, F}(U)$. We define $\operatorname{det}^{\Gamma} R \Gamma_{c}(U, \mathcal{M})$ to be

$$
\lim _{\left(\mathcal{E}_{X}, \overleftarrow{\nabla}\right): \text { small }}\left(\operatorname{det} R \Gamma\left(X, D R\left(\mathcal{E}_{X}\right)\right), \operatorname{det} R \Gamma_{c}\left(U^{a n}, V\right), \Gamma\left(\nabla: \mathcal{E}_{X}\right)^{-1} \times \operatorname{det} R \Gamma_{c}(U, \rho)\right)
$$


Here the transition map $\operatorname{det} R \Gamma\left(X, D R\left(\mathcal{E}_{X}^{\prime}\right)\right) \rightarrow \operatorname{det} R \Gamma\left(X, D R\left(\mathcal{E}_{X}\right)\right)$ for small extensions $\mathcal{E}_{X}^{\prime} \subset \mathcal{E}_{X}$ of $\mathcal{E}$ is $\operatorname{det}\left(\nabla, \mathcal{E}_{X} / \mathcal{E}_{X}^{\prime}\right) \in k^{\times}$times the natural map induced by the quasi-isomorphism $D R\left(\mathcal{E}_{X}^{\prime}\right) \rightarrow D R\left(\mathcal{E}_{X}\right)$. They form an inverse system by Lemma 1.8. The isomorphism class of $\operatorname{det}^{\Gamma} R \Gamma_{c}(U, \mathcal{M})$ is $\operatorname{per}_{c}^{\Gamma}(\mathcal{M})=\left[\operatorname{det}^{\Gamma} R \Gamma_{c}(U, \mathcal{M})\right] \in$ $M \operatorname{Pic}_{k_{0}, F}(k)$. We let

$$
\operatorname{det}^{\Gamma} R \Gamma_{c}(U, D R(\mathcal{E}))=\varliminf_{\left(\mathcal{E}_{X}, \overleftarrow{\nabla}\right): \text { small }} \operatorname{det} R \Gamma\left(X, D R\left(\mathcal{E}_{X}\right)\right)
$$

denote the de Rham component of $\operatorname{det}^{\Gamma} R \Gamma_{c}(U, \mathcal{M})$ which is a $k$-vector space of dimension 1.

To define $\left(\operatorname{det} \mathcal{M}, \mathbf{c}_{X \bmod D}\right)$, we refine the theory of tame symbol in Section 3. For a pair of an invertible $\mathcal{O}_{X}$-module $\mathcal{L}$ and an isomorphism $r: \mathcal{L} \otimes_{\mathcal{O}_{X}} \mathcal{O}_{D} \rightarrow \mathcal{O}_{D}$, we define a functor $(,(\mathcal{L}, r)): P_{k_{0}, F}(U) \rightarrow P_{k_{0}, F}(k)$ called the tame symbol. Applying this functor to $\operatorname{det} \mathcal{M}\left(\mathcal{M} \in M_{k_{0}, F}(U)\right)$ and the dual $\mathbf{c}_{X \bmod D}=\left(\Omega_{X}^{1}(\log D) \text {, res }\right)^{*}$ of $\left(\Omega_{X}^{1}(\log D)\right.$, res), we will define the tame symbol $\left(\operatorname{det} \mathcal{M}, \mathbf{c}_{X \bmod D}\right) \in P_{k_{0}, F}(k)$. We let $\left(\operatorname{det} \mathcal{E}, \mathbf{c}_{X \bmod D}\right)$ denote its de Rham component. Before defining the tame symbol, we define the norm functor $N_{L / k}: P_{k_{0}, F}(L) \rightarrow P_{k_{0}, F}(k)$ and the tame symbol $(, f)_{x}$ for $x \in D$ and $f \in A_{x}$.

For a finite extension $L$ of $k$, we define the norm functor $N_{L / k}: P_{k_{0}, F}(L) \rightarrow$ $P_{k_{0}, F}(k)$. Let $\mathcal{M}=(E, V, \rho) \in P_{k_{0}, F}(L)$ and we define its norm $N_{L / k}(\mathcal{M})=$ $(N E, N V, N \rho) \in P_{k_{0}, F}(k)$. The $k$-vector space $N E$ of dimension 1 is given in [D7], 7.1. For a basis $e$ of $E$, the vector space $N E$ has the symbol $N e$ as a basis. For another basis $e^{\prime}=f e, f \in L^{\times}$, we require $N e^{\prime}=N_{L / k} f N e$. The constant sheaf $N V$ of $F$-vector spaces of dimension 1 on Spec $k^{a n}$ is defined similarly. For a basis $v$ of $V$, the constant sheaf $N V$ has the symbol $N v$ as a basis. For another basis $v^{\prime}=f v, f \in\left(F^{\times}\right)^{H_{o m} m_{k_{0}}(L, \mathbb{C})}$, we impose $N v^{\prime}=N_{L / k} f N v$. For $\sigma: k \rightarrow \mathbb{C}$, $N_{L / k} f_{\sigma}=\prod_{\tau:\left.\tau\right|_{k}=\sigma} f_{\tau}$, the comparison map $N \rho$ is defined by $N \rho(N v)=N_{X / Y} \varphi N e$ for $\varphi=\rho(v) / e$.

For $x \in U$, the restriction $\left.\mathcal{M}\right|_{x}$ denotes the triple $\left(\mathcal{E} \otimes \kappa(x),\left.V\right|_{x^{a n}},\left.\rho\right|_{x}\right) \in P_{k_{0}, F}(x)$ and $N_{x / k}\left(\left.\mathcal{M}\right|_{x}\right) \in P_{k_{0}, F}(k)$ denotes its norm.

Let $x \in D$ and $f \in A_{x}$. We define the tame symbol functor $(, f)_{x}: P_{k_{0}, F}(U) \rightarrow$ $P_{k_{0}, F}(x)$. Let $\mathcal{M}=((\mathcal{E}, \nabla), V, \rho) \in P_{k_{0}, F}(U)$ and we define the tame symbol $(\mathcal{M}, f)=((\mathcal{E}, f),(V, f),(\rho, f)) \in P_{k_{0}, F}(x)$. We define a $\kappa(x)$-vector space $(\mathcal{E}, f)$ of dimension 1. For a non-zero rational section $e$ of $\mathcal{E}$ the vector space $(\mathcal{E}, f)$ has the symbol $(e, f)$ as a basis. For another basis $e^{\prime}=g e, g \in k(X)^{\times}$, we require $\left(e^{\prime}, f\right)=(g, f)(e, f)$ where $(g, f)=(-1)^{\operatorname{ord}_{x} f \operatorname{ord}_{x} g} g^{\operatorname{ord}_{x} f} f^{-\operatorname{ord}_{x} g}$ is the classical tame symbol. For an extension $\mathcal{E}_{X}$ of $\mathcal{E}$, there is a canonical isomorphism $(\mathcal{E}, f) \rightarrow\left(\mathcal{E}_{X} \otimes \kappa(x)\right)^{\operatorname{ord}_{x} f}:(e, f) \mapsto e^{\otimes \operatorname{ord}_{x} f}$ for a local basis $e$ of $\mathcal{E}_{X}$ at $x$ which is independent of the choice of $e$. The constant sheaf $(V, f)$ of $F$-vector spaces of dimension 1 is the restriction at Spec $k^{a n}$ of the unramified subsheaf $(V, \tilde{f})^{\sim}=$ $(-1)^{-\operatorname{ord}_{x} f \cdot \nabla_{x}}(\tilde{f})^{\nabla_{x}} V^{\otimes \operatorname{ord}_{x} f} \subset V^{\otimes \operatorname{ord}_{x} f} \otimes_{F} \mathcal{O}_{X}^{a n}$ defined in Section 3. Here $\nabla_{x}$ is the residue of $\mathcal{E}_{X}$ at $x$ and $\tilde{f} \in k(X)^{\times}$is a lifting of $f \in A_{x}$. The comparison map $(\rho, f)$ is induced by the restriction of $(V, \tilde{f})^{\sim} \subset V^{\otimes \operatorname{ord}_{x} f} \otimes_{F} \mathcal{O}_{X}^{a n} \stackrel{\rho}{\rightarrow} \mathcal{E}_{X}^{a n \otimes \operatorname{ord}_{x} f}$. Thus a triple $(\mathcal{M}, f)=((\mathcal{E}, f),(V, f),(\rho, f)) \in P_{k_{0}, F}(x)$ is defined and is independent of the choices made in the definition.

Definition 17. Let $(\mathcal{L}, r)$ be as above and $\ell$ be a non-zero rational section of $\mathcal{L}$. For an object $\mathcal{M}=((\mathcal{E}, \nabla), V, \rho)$ of $P_{k_{0}, F}(U)$, we will define $(\mathcal{M}, \ell)$ as the tensor 
product

$$
\bigotimes_{x \in U} N_{x / k}\left(\left.\mathcal{M}\right|_{x}\right)^{\operatorname{ord}_{x} \ell} \otimes \bigotimes_{x \in D} N_{x / k}\left(\mathcal{M}, f_{x}\right)_{x}
$$

where $f_{x} \in A_{x}=k(X)^{\times} / 1+m_{x}$ is the class of a rational function $\tilde{f}_{x}$ satisfying that $\operatorname{ord}_{x} \ell=\operatorname{ord}_{x} \tilde{f}_{x}$ and $r_{x}\left(\tilde{f}_{x}^{-1} \ell\right)=1$.

We defined a functor $(, \ell): P_{k_{0}, F}(U) \rightarrow P_{k_{0}, F}(k)$ for a non-zero rational section $\ell$ of $\mathcal{L}$. We will define an isomorphism of the functor $(, f):(, \ell) \rightarrow(, f \ell)$ for a non-zero rational function $f$ on $X$ satisfying $(, f g)=(, f) \circ(, g)$ in Proposition 5. Therefore, we get the functor $(,(\mathcal{L}, r))$ as the limit of the functors $(, \ell)$ with respect to the transition isomorphisms $(, f)$.

Thus each term in (1) is defined and the tame symbol functor $(, \ell)$ is defined as the tensor product. The reciprocity law, Proposition 2 in Section 3, is refined as follows.

Proposition 5. Let $U$ be a smooth curve, $X$ be the compactification and $D=$ $X-U$ be the complement. Let $\mathcal{L}$ be an invertible $\mathcal{O}_{X}$-module with a trivialization $r: \mathcal{L} \otimes \mathcal{O}_{D} \rightarrow \mathcal{O}_{D}$. For a non-zero rational section $\ell$ of $\mathcal{L}$ and a non-zero rational function $f$ on $X$, there is a canonical isomorphism of the functor $(, f):(, \ell) \rightarrow$ $(, f \ell)$ satisfying $(, f g)=(, f) \circ(, g)$ for another non-zero rational function $g$.

Proof. By considering a rational function $f$ as a rational section of $\mathcal{O}_{X}$, it is reduced to the case $\mathcal{L}=\mathcal{O}_{X}$. Since a morphism in $M_{k_{0}, F}(U)$ is determined by its de Rham component, it is enough to prove

Proposition $5^{\prime}$. For $\mathcal{M}=((\mathcal{E}, \nabla), V, \rho) \in P_{k_{0}, F}(U)$, the isomorphism

$$
(\mathcal{E}, f) \rightarrow k:(e, f)=\bigotimes_{x \in U} N\left(\left.(-1)^{\operatorname{ord}_{x} e \cdot \operatorname{ord}_{x} f} e^{\otimes \operatorname{ord}_{x} f} f^{-\operatorname{ord}_{x} e}\right|_{x}\right) \otimes \bigotimes_{x \in D} N(e, f)_{x} \mapsto 1
$$

for a non-zero rational section $e$ of $\mathcal{E}$ is independent of the choice of $e$ and defines an isomorphism of functors $(, f) \rightarrow \mathbf{1}$ where $\mathbf{1}$ is the constant functor.

By Weil's reciprocity law $(g, f)=\prod_{x}(g, f)_{x}=1$, the isomorphism is independent of the choice of $e$. Since the morphism of $M_{k_{0}, F}(k)$ is determined by the de Rham component, it is enough to show that the map on the de Rham component defined above induces an isomorphism in $M_{k_{0}, F}(k)$.

When $f$ is a constant or the coordinate $t$ of $\mathbb{P}_{k}^{1}$, the argument in the proof as for Proposition 2 in Section 3 shows Proposition $5^{\prime}$. Let $f$ be an arbitrary nonconstant function and $f: X \rightarrow \mathbb{P}^{1}$ be the map defined by $f$. We compare $(\mathcal{M}, f)$ and $(N \mathcal{M}, t)$. We define an isomorphism $(\mathcal{E}, f) \rightarrow(N \mathcal{E}, t)$ of 1 -dimensional $k$-vector spaces as the tensor product of

$$
\bigotimes_{x \mapsto y} N_{x / y}(\mathcal{E}, f)_{x} \rightarrow(N \mathcal{E}, t)_{y}: \bigotimes_{x \mapsto y}(e, f)_{x} \mapsto \bigotimes_{x \mapsto y}(e, f)_{x} .
$$

It is compatible with the trivialization in the statement. The proof of the Claim in Section 3 shows that the isomorphism (1) induces an isomorphism

$$
\bigotimes_{x \mapsto y} N_{x / y}(\mathcal{M}, f)_{x} \rightarrow(N \mathcal{M}, t)_{y} .
$$

Hence the above isomorphism $(\mathcal{E}, f) \rightarrow(N \mathcal{E}, t)$ induces an isomorphism $(\mathcal{M}, f) \rightarrow$ $(N \mathcal{M}, t)$. Since it is proved already for $X=\mathbb{P}^{1}$ and $f=t$, Proposition $5^{\prime}$ follows. 
For an integrable connection $(\mathcal{E}, \nabla)$ of rank $r$ on $U$ regular along $D$, we define a canonical isomorphism

$$
D R R: \operatorname{det}^{\Gamma} R \Gamma_{c}(U, D R(\mathcal{E})) \otimes \operatorname{det}^{\Gamma} R \Gamma_{c}\left(U, D R\left(\mathcal{O}_{U}\right)\right)^{\otimes-r} \simeq\left(\operatorname{det} \mathcal{E}, \mathbf{c}_{X \bmod D}\right)
$$

using the isomorphism of Deligne-Riemann-Roch. Take an extension $\left(\mathcal{E}_{X}, \nabla\right)$ of $(\mathcal{E}, \nabla)$. By the isomorphism of the pairings above, we identify $\left(\operatorname{det} \mathcal{E}, \mathbf{c}_{X \bmod D}\right)=$ $\left(\operatorname{det} \mathcal{E}_{X}(-r D), \Omega_{X}^{1}(\log D)^{\otimes-1}\right)$. We define a canonical isomorphism

$$
\begin{aligned}
\operatorname{det} R \Gamma\left(X, D R\left(\mathcal{E}_{X}\right)\right) & \otimes \operatorname{det} R \Gamma\left(X, D R\left(\mathcal{O}_{X}(-D)\right)\right)^{\otimes-r} \\
& \simeq\left(\operatorname{det} \mathcal{E}_{X}(-r D), \Omega_{X}^{1}(\log D)^{\otimes-1}\right) .
\end{aligned}
$$

Here $D R\left(\mathcal{O}_{X}(-D)\right)=\left[\mathcal{O}_{X}(-D) \stackrel{d}{\rightarrow} \mathcal{O}_{X}(-D) \otimes \Omega_{X}^{1}(\log D)\right]$. In the notation of [D7], the left hand side is

$$
\operatorname{det} R \Gamma\left(X,\left(\left[\mathcal{E}_{X}\right]-r\left[\mathcal{O}_{X}(-D)\right]\right) \otimes\left(\left[\mathcal{O}_{X}\right]-\left[\Omega_{X}^{1}(\log D)\right]\right)\right)
$$

Therefore by Deligne-Riemann-Roch (Construction 7.2 loc.cit.), it is canonically isomorphic to $\left(\operatorname{det} \mathcal{E}_{X}(-r D), \Omega_{X}^{1}(\log D)^{\otimes-1}\right)$.

We write $\lambda\left(\mathcal{E}_{X}\right)=\operatorname{det} R \Gamma\left(X, D R\left(\mathcal{E}_{X}\right)\right)$ for short.

Lemma 6.1. Let $\mathcal{E}_{X}^{\prime} \subset \mathcal{E}_{X}$ be extensions. Assume the canonical map $\operatorname{DR}\left(\mathcal{E}_{X}^{\prime}\right) \rightarrow$ $\operatorname{DR}\left(\mathcal{E}_{X}\right)$ is a quasi-isomorphism. Then the diagram

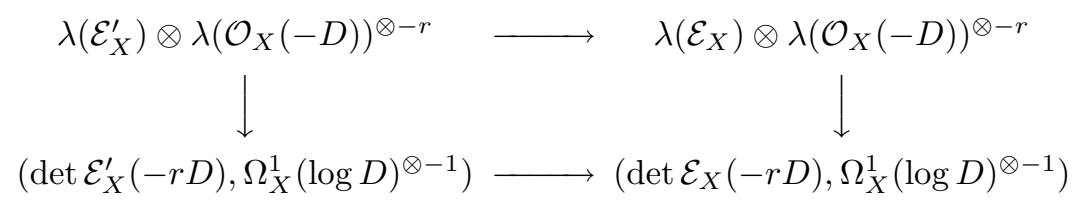

is commutative. Here the upper horizontal map is $\operatorname{det}\left(\operatorname{res} \nabla ; \mathcal{E}_{X} / \mathcal{E}_{X}^{\prime}\right)$-times the isomorphism induced by the quasi-isomorphism $D R\left(\mathcal{E}_{X}^{\prime}\right) \rightarrow D R\left(\mathcal{E}_{X}\right)$ and the lower one is defined by the isomorphism $\left.\mathcal{E}_{X}^{\prime}\right|_{U}=\left.\mathcal{E}_{X}\right|_{U}$. The vertical arrows are defined by Deligne-Riemann-Roch.

Definition 18. By the above Lemma, the isomorphisms are compatible with the change of small extensions $\mathcal{E}_{X}$ and induce an isomorphism

$$
D R R: \operatorname{det}^{\Gamma} R \Gamma_{c}(U, D R(\mathcal{E})) \otimes \operatorname{det}^{\Gamma} R \Gamma_{c}\left(U, D R\left(\mathcal{O}_{U}\right)\right)^{\otimes-r} \simeq\left(\operatorname{det} \mathcal{E}, \mathbf{c}_{X \bmod D}\right) .
$$

Proof. By induction on the length of $\mathcal{E}_{X} / \mathcal{E}_{X}^{\prime}$, we may assume $\mathcal{F}=\mathcal{E}_{X} / \mathcal{E}_{X}^{\prime}$ is a $\kappa(x)$-vector space for some $x \in D$ and $\operatorname{det}\left(\nabla: \mathcal{E}_{X} / \mathcal{E}_{X}^{\prime}\right)=N_{x / k}\left(\operatorname{det}\left(\operatorname{res}_{x} \nabla: \mathcal{F}\right)\right)$. By definition of the isomorphisms, it is sufficient to show the commutativity of the diagram

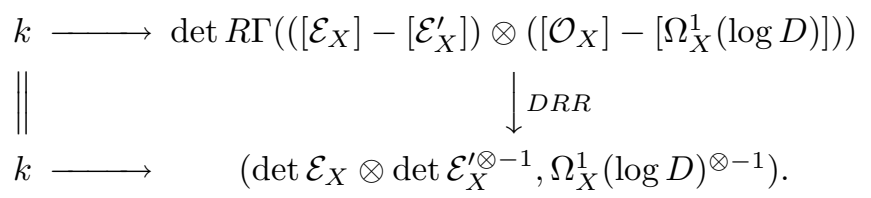

Here the upper horizontal arrow is $\operatorname{det}\left(\nabla: \mathcal{E}_{X} / \mathcal{E}_{X}^{\prime}\right)$-times the isomorphism induced by the isomorphism $\nabla: \mathcal{E}_{X} / \mathcal{E}_{X}^{\prime} \rightarrow \mathcal{E}_{X} / \mathcal{E}_{X}^{\prime} \otimes \Omega_{X}^{1}(\log D)$ and the lower one is defined by the isomorphism $\left.\mathcal{E}_{X}\right|_{U}=\left.\mathcal{E}_{X}^{\prime}\right|_{U}$. By the definition of the isomorphism of Deligne-Riemann-Roch 7.1 and 7.2 loc.cit., the diagram is commutative if we replace the upper horizontal arrow by the isomorphism induced by $i d \otimes \operatorname{res}_{x}^{-1}: \mathcal{E}_{X} / \mathcal{E}_{X}^{\prime}$ $\rightarrow \mathcal{E}_{X} / \mathcal{E}_{X}^{\prime} \otimes \Omega_{X}^{1}(\log D)$. The assertion follows from this and the equality $\operatorname{det}\left(\nabla: \mathcal{E}_{X} / \mathcal{E}_{X}^{\prime}\right)=N_{x / k}\left(\operatorname{det}\left(\operatorname{res}_{x} \nabla: \mathcal{F}\right)\right)$. 


\section{(b) Main result.}

Theorem 3. Let $X$ be a proper smooth curve over $k$ and $\mathcal{M} \in M_{k_{0}, F}(U)$ on a open subscheme $U \subset X$. Then the morphisim DRR (Definition 18) induces an isomorphism

$$
\operatorname{det}^{\Gamma} R \Gamma_{c}(U, \mathcal{M}) \otimes \operatorname{det}^{\Gamma} R \Gamma_{c}(U, \mathbf{1})^{\otimes-\operatorname{rank} \mathcal{M}} \simeq\left(\operatorname{det} \mathcal{M}, \mathbf{c}_{X \bmod D}\right)
$$

in $P_{k_{0}, F}(k)$.

We deduce Theorem 3 for $X=\mathbb{P}_{k}^{1}$ from Theorem $\mathrm{T}$ in Section 4 and then prove it for general $X$ by taking a finite map $X \rightarrow \mathbb{P}_{k}^{1}$. Because the idea of the proof is the same as that of Theorem 1, we only give arguments which require special care for isomorphisms.

Remark. For an arbitrary dimension, it seems possible to define a canonical isomorphism of Deligne-Riemann-Roch:

$$
\begin{aligned}
\operatorname{det}^{\Gamma} R \Gamma\left(X, D R\left(\mathcal{E}_{X}\right)\right) & \otimes\left(\operatorname{det}^{\Gamma} R \Gamma\left(X, D R\left(\mathcal{O}_{X}(-D)\right)\right)\right)^{\otimes-r} \\
& \simeq\left(\operatorname{det} \mathcal{E}, \mathbf{c}_{X \bmod D}\right) .
\end{aligned}
$$

Note that $\operatorname{ch}\left(\left[D R\left(\mathcal{E}_{X}\right)\right]-r\left[D R\left(\mathcal{O}_{X}(-D)\right)\right]\right)=c_{1}\left(\mathcal{E}_{X}(-D)\right) \cdot c_{n}\left(\Omega_{X}^{1}(\log D)\right)$ modulo higher terms. It should induce an isomorphism

$$
\operatorname{det}^{\Gamma} R \Gamma_{c}(U, \mathcal{M}) \otimes \operatorname{det}^{\Gamma} R \Gamma_{c}(U, \mathbf{1})^{\otimes-r} \simeq\left(\operatorname{det} \mathcal{M}, \mathbf{c}_{X \bmod D}\right)
$$

as in Theorem 3 for curves by a similar argument.

Proof of Theorem 3. Similarly as Lemma 4.1, we may shrink $U$.

Lemma 6.2. Let $x \in U$ be a closed point and put $U^{\prime}=U-\{x\}$. Then Theorem 3 for $\mathcal{M}$ and $\left.\mathcal{M}\right|_{U^{\prime}}$ are equivalent.

Proof. By the proof of Lemma 4.1, it is enough to check the commutativity of the diagram of isomorphisms

$$
\begin{gathered}
\lambda_{X}\left(\mathcal{E}_{X}\right) \otimes \lambda_{X}\left(\mathcal{O}_{X}(-D)\right)^{\otimes-r} \longrightarrow \begin{array}{r}
\lambda_{X}^{\prime}\left(\mathcal{E}_{X}(-x)\right) \otimes \lambda_{X}^{\prime}\left(\mathcal{O}_{X}\left(-D^{\prime}\right)\right)^{\otimes-r} \\
\otimes \operatorname{det}_{k}(\mathcal{E} \otimes \kappa(x)) \otimes \operatorname{det}_{k}(\kappa(x))^{\otimes-r} \\
\downarrow \\
\left(\operatorname{det} \mathcal{E}, \mathbf{c}_{X \bmod D}\right)
\end{array} \quad \longrightarrow\left(\left.\operatorname{det} \mathcal{E}\right|_{U^{\prime}}, \mathbf{c}_{X \bmod D^{\prime}}\right) \otimes N_{x / k}(\operatorname{det} \mathcal{E} \otimes \kappa(x))
\end{gathered}
$$

where $D^{\prime}=D \cup\{x\}$ and $\lambda_{X}^{\prime}$ denotes the determinant for the de Rham complex defined by $\Omega_{X}^{1}\left(\log D^{\prime}\right)$. The vertical isomorphisms in the diagram are given by the isomorphisms

$$
\begin{gathered}
\operatorname{det} R \Gamma\left(X,\left(\left[\mathcal{E}_{X}\right]-r\left[\mathcal{O}_{X}(-D)\right]\right) \otimes\left(\left[\mathcal{O}_{X}\right]-\left[\Omega_{X}^{1}(\log D)\right]\right)\right) \\
\rightarrow\left(\operatorname{det} \mathcal{E}_{X}(-r D), \Omega_{X}^{1}(\log D)^{\otimes-1}\right), \\
\operatorname{det} R \Gamma\left(X,\left(\left[\mathcal{E}_{X}(-x)\right]-r\left[\mathcal{O}_{X}\left(-D^{\prime}\right)\right]\right) \otimes\left(\left[\mathcal{O}_{X}\right]-\left[\Omega_{X}^{1}\left(\log D^{\prime}\right)\right]\right)\right) \\
\rightarrow\left(\operatorname{det} \mathcal{E}_{X}(-r D), \Omega_{X}^{1}\left(\log D^{\prime}\right)^{\otimes-1}\right), \\
\operatorname{det} R \Gamma\left(X,\left(\left[\mathcal{E}_{X}\right]-r\left[\mathcal{O}_{X}(-D)\right]\right) \otimes\left(\left[\Omega_{X}^{1}\left(\log D^{\prime}\right)\right]-\left[\Omega_{X}^{1}(\log D)\right]\right)\right) \\
\rightarrow\left(\operatorname{det} \mathcal{E}_{X}(-r D), \mathcal{O}_{X}(x)\right)=N_{x / k}(\mathcal{E} \otimes \kappa(x))
\end{gathered}
$$


Since the second isomorphism is the same as

$$
\begin{aligned}
\operatorname{det} R \Gamma\left(X,\left(\left[\mathcal{E}_{X}\right]-r\left[\mathcal{O}_{X}(-D)\right]\right)\right. & \left.\otimes\left(\left[\mathcal{O}_{X}\right]-\left[\Omega_{X}^{1}\left(\log D^{\prime}\right)\right]\right)\right) \\
& \rightarrow\left(\operatorname{det} \mathcal{E}_{X}(-r D), \Omega_{X}^{1}\left(\log D^{\prime}\right)^{\otimes-1}\right),
\end{aligned}
$$

the diagram is commutative.

We deduce Theorem 3 for $\mathbb{P}_{k}^{1}$ from Theorem $\mathrm{T}$.

Proof of Theorem 3 for $X=\mathbb{P}_{k}^{1}$. First, we give a refinement of Claim 1 in subsection 4(b). Let the notation $U^{\prime}=U-\{\infty\}$ etc. be as in loc.cit. For an object $\mathcal{M}=((\mathcal{E}, \nabla), V, \rho)$ of $M_{k_{0}, F, X}\left(U^{\prime}\right)$, we define a variant $\operatorname{det}^{\Gamma} R \Gamma^{\prime}\left(U^{\prime}, \mathcal{M}\right)$ as the triple

$$
\varliminf_{\left(\mathcal{E}_{X}, \nabla\right)}^{\lim }\left(\operatorname{det} R \Gamma\left(X, D R\left(\mathcal{E}_{X}\right)\right), \operatorname{det} R \Gamma\left(A^{a n}, j_{!} V\right), \Gamma^{\prime}\left(\nabla: \mathcal{E}_{X}\right)^{-1} \times \operatorname{det} R \Gamma(\rho)\right)
$$

$\in P_{k_{0}, F}(k)$ similarly as in the definition of $\operatorname{det}^{\Gamma} R \Gamma_{c}\left(U^{\prime}, \mathcal{M}\right)$. Here the extensions $\left(\mathcal{E}_{X}, \nabla\right)$ of $(\mathcal{E}, \nabla)$ are small at $D$ and big at $\infty$ and the transition morphism for $\mathcal{E}_{X}^{\prime} \subset \mathcal{E}_{X}$ is $\left(\operatorname{det}\left(\nabla: \mathcal{E}_{X} / \mathcal{E}_{X}^{\prime}\right)\right)$-times the isomorphism induced by the inclusion. Recall that, for an extension $\mathcal{E}_{X}$ of $\mathcal{E}$, we put

$$
\Gamma^{\prime}\left(\nabla: \mathcal{E}_{X}\right)=\prod_{x \in D} N_{x / k} \Gamma\left(\operatorname{res}_{x} \nabla\right) \times(-1)^{\operatorname{Tr}\left(\nabla_{\infty}\right)} \Gamma\left(1-\operatorname{res}_{\infty} \nabla\right)^{-1}
$$

$\in\left(k \otimes_{k_{0}} \mathbb{C}\right)^{\times}$. By the equality $s(-1)^{-(s-1)} \Gamma(1-(s-1))=(-1)^{-s} \Gamma(1-s)$, they form a projective system.

We show Theorem $\mathrm{T}$ implies

Claim 1. Assume $\infty \in U$. Let $\mathcal{M}=((\mathcal{E}, \nabla), V, \rho)$ be an object of $M_{k_{0}, F, X}(U)$. Assume that there exists an extension $\left(\mathcal{E}_{X}, \nabla\right)$ of $(\mathcal{E}, \nabla)$ such that $\mathcal{E}_{X} \simeq \mathcal{O}_{X}(-m)^{r}$ for some $m \in \mathbb{Z}$. Then the isomorphism of Deligne-Riemann-Roch

$$
\begin{aligned}
\operatorname{det} R \Gamma\left(X, D R\left(\mathcal{E}_{X}\right)\right) & \otimes \operatorname{det} R \Gamma\left(X, D R\left(\mathcal{O}_{X}(-D)\right)\right)^{\otimes-r} \\
& \simeq\left(\operatorname{det} \mathcal{E}_{X}(-r D), \Omega_{X}^{1}\left(\log D^{\prime}\right)^{\otimes-1}\right)
\end{aligned}
$$

induces an isomorphism

$$
\operatorname{det}^{\Gamma} R \Gamma^{\prime}\left(U^{\prime},\left.\mathcal{M}\right|_{U^{\prime}}\right) \otimes \operatorname{det}^{\Gamma} R \Gamma^{\prime}\left(U^{\prime}, \mathbf{1}\right)^{\otimes-r} \simeq\left(\left.\operatorname{det} \mathcal{M}\right|_{U^{\prime}}, \mathbf{c}_{X \bmod D^{\prime}}\right)
$$

in $P_{k_{0}, F}(k)$.

Proof of Claim 1. By the same argument as in Section 4, we may assume $\mathcal{E}_{X} \simeq \mathcal{O}_{X}^{r}$ and the condition (2) loc.cit. is verified. It is sufficient to show that for each embedding $k \rightarrow \mathbb{C}$ over $k_{0}$, there is an isomorphism

$$
\operatorname{det} R \Gamma\left(A^{a n}, j_{!} V\right) \otimes \operatorname{det} R \Gamma\left(A^{a n}, j_{!} F\right)^{\otimes-r} \rightarrow\left(\operatorname{det} V \otimes F^{\otimes-r}, \mathbf{c}_{X \bmod D^{\prime}}\right)
$$

of $F$-vector spaces such that the diagram

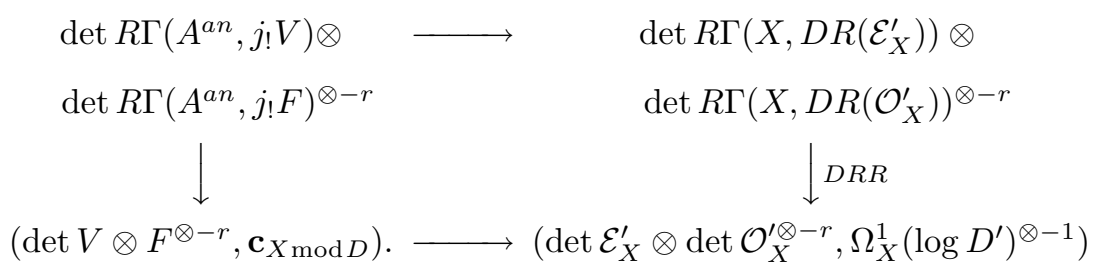


is commutative. Here the upper horizontal arrow is

$$
\Gamma^{\prime}\left(\nabla: \mathcal{E}_{X}\right)^{-1} \times \Gamma^{\prime}\left(\nabla: \mathcal{O}_{X}(-D)\right)^{r} \times \operatorname{det} R \Gamma(\rho) \otimes \operatorname{det} R \Gamma(\text { canonical })^{\otimes-r}
$$

and the lower one is the comparison map of $\left(\left.\operatorname{det} \mathcal{M}\right|_{U^{\prime}}, \mathbf{c}_{X \bmod D^{\prime}}\right)$.

By the definition of the tame symbol given in Section 3 and by the definition of the map $(\operatorname{det} \rho, d t)$ (and $(\operatorname{det} \operatorname{can}, d t)$ ) in Theorem $\mathrm{T}$, we have a commutative diagram

$$
\begin{array}{cc}
\left(\operatorname{det} \bigoplus_{x \in D^{a n}} V_{b_{x}}\right)^{\otimes-1} \otimes \operatorname{det} V_{b_{\infty}} \otimes & \operatorname{det}\left(\left.\mathcal{E}_{X}^{\prime}\right|_{D}\right)^{\otimes-1} \otimes \operatorname{det}\left(\mathcal{E}_{X}^{\prime}(\infty)\right) \otimes \\
\left(\left(\operatorname{det} \bigoplus_{x \in D^{a n}} F\right)^{\otimes-1} \otimes F\right)^{\otimes-r} & \left(\operatorname{det}\left(\left.\mathcal{O}_{X}^{\prime}\right|_{D}\right)^{\otimes-1} \otimes \operatorname{det}\left(\mathcal{O}_{X}^{\prime}(\infty)\right)\right)^{\otimes-r} \\
\downarrow & \downarrow \\
\left(\operatorname{det} V \otimes F^{\otimes-r}, \mathbf{c}_{X \bmod D^{\prime}}\right) & \longrightarrow\left(\operatorname{det} \mathcal{E}_{X}^{\prime} \otimes \operatorname{det} \mathcal{O}_{X}^{\prime \otimes-r}, \Omega_{X}^{1}\left(\log D^{\prime}\right)^{\otimes-1}\right) .
\end{array}
$$

Here the upper horizontal arrow is $(\operatorname{det} \rho, d t) \otimes(\operatorname{det} \text { can }, d t)^{\otimes-r}$ and the lower one is $\left(\operatorname{det} \rho \otimes \operatorname{can}^{\otimes-r}, \mathbf{c}_{X \bmod D^{\prime}}\right)$. The left vertical arrow is induced by the tame symbols $(, t-t(x))_{x}$ for $x \in D^{a n}$ and $\left(,-t^{-1}\right)_{\infty}$ and the right one is defined by a property of the norm $\operatorname{det}\left(\left.\mathcal{E}_{X}^{\prime}\right|_{D}\right) \otimes \operatorname{det}\left(\left.\mathcal{O}_{X}^{\prime}\right|_{D}\right)^{\otimes-r} \simeq N_{D / k}\left(\operatorname{det}\left(\left.\mathcal{E}_{X}^{\prime}\right|_{D}\right)\right)$ and the isomorphism

$$
\begin{gathered}
N_{D / k}\left(\operatorname{det}\left(\left.\mathcal{E}_{X}^{\prime}\right|_{D}\right)\right) \otimes\left(\operatorname{det}\left(\mathcal{E}_{X}^{\prime}(\infty)\right) \otimes \operatorname{det}\left(\mathcal{O}_{X}^{\prime}(\infty)\right)^{\otimes-r}\right) \\
\rightarrow\left(\operatorname{det} \mathcal{E}_{X}^{\prime} \otimes \operatorname{det} \mathcal{O}_{X}^{\prime \otimes-r}, \Omega_{X}^{1}\left(\log D^{\prime}\right)^{\otimes-1}\right) .
\end{gathered}
$$

The isomorphism of Deligne-Riemann-Roch is the composition of this and

$$
\begin{gathered}
\operatorname{det} R \Gamma\left(X, D R\left(\mathcal{E}_{X}^{\prime}\right)\right) \otimes \operatorname{det} R \Gamma\left(X, D R\left(\mathcal{O}_{X}^{\prime}\right)\right)^{\otimes-r} \\
\stackrel{\beta_{\mathcal{E}_{X}^{\prime}} \otimes \beta_{\mathcal{O}_{X}^{\prime}}^{\otimes-r}}{\longrightarrow} \operatorname{det}\left(\left.\mathcal{E}_{X}^{\prime}\right|_{D}\right)^{\otimes-1} \otimes \operatorname{det}\left(\mathcal{E}_{X}^{\prime}(\infty)\right) \otimes\left(\operatorname{det}\left(\left.\mathcal{O}_{X}^{\prime}\right|_{D}\right)^{\otimes-1} \otimes \operatorname{det}\left(\mathcal{O}_{X}^{\prime}(\infty)\right)\right)^{\otimes-r} .
\end{gathered}
$$

Therefore by Theorem $\mathrm{T}$ for $\mathcal{E}_{X}^{\prime}$ and $\mathcal{O}_{X}^{\prime}$, the composite of $\alpha_{V} \otimes \alpha_{F}^{\otimes-r}$ and the left vertical arrow of the diagram above gives the required isomorphism of the $F$-vector spaces

$$
\operatorname{det} R \Gamma\left(A^{a n}, j_{!} V\right) \otimes \operatorname{det} R \Gamma\left(A^{a n}, j_{!} F\right)^{\otimes-r} \rightarrow\left(\operatorname{det} V \otimes F^{\otimes-r},\left(\Omega_{X}^{1}\left(\log D^{\prime}\right), \operatorname{res}\right)^{*}\right) .
$$

Thus Claim 1 is proved.

We prove Theorem 3 for $X=\mathbb{P}^{1}$. Let $\mathcal{M}=((\mathcal{E}, \nabla), V, \rho)$ be an object of $M_{k_{0}, F, X}(U)$. As in Section 4, by shrinking $U$, we may take an extension $\mathcal{E}_{X}$ of $\mathcal{E}$ as in the assumption of Claim 1. It is sufficient to define canonical isomorphisms

$$
\begin{gathered}
\operatorname{det}^{\Gamma} R \Gamma_{c}(U, \mathcal{M}) \simeq \operatorname{det}^{\Gamma} R \Gamma^{\prime}\left(U^{\prime},\left.\mathcal{M}\right|_{U^{\prime}}\right) \otimes \operatorname{det}\left(\left.\mathcal{M}\right|_{\infty}(-1)\right), \\
\left(\operatorname{det} \mathcal{M}, \mathbf{c}_{X \bmod D^{\prime}}\right) \simeq\left(\left.\operatorname{det} \mathcal{M}\right|_{U^{\prime}}, \mathbf{c}_{X \bmod D^{\prime}}\right) \otimes \operatorname{det}\left(\left.\mathcal{M}\right|_{\infty}\right)
\end{gathered}
$$

satisfying the following condition.

The de Rham components of the isomorphisms form a commutative diagram

$$
\begin{aligned}
& \lambda\left(\mathcal{E}_{X}\right) \otimes \lambda\left(\mathcal{O}_{X}(-D)\right)^{\otimes-r} \rightarrow \lambda^{\prime}\left(\mathcal{E}_{X}\right) \otimes \lambda^{\prime}\left(\mathcal{O}_{X}(-D)\right)^{\otimes-r} \otimes \operatorname{det} \mathcal{E}_{X}(\infty) \\
& \qquad D R R \downarrow \\
& \left(\operatorname{det} \mathcal{E}_{X}(-r D), \Omega_{X}^{1}(\log D)^{\otimes-1}\right) \rightarrow\left(\operatorname{det} \mathcal{E}_{X}(-r D), \Omega_{X}\left(\log D^{\prime}\right)^{\otimes-1}\right) \otimes \operatorname{det} \mathcal{E}_{X}(\infty) \\
& \text { for small extensions }\left(\mathcal{E}_{X}, \nabla\right) .
\end{aligned}
$$


Here we write

$$
\begin{aligned}
\lambda\left(\mathcal{E}_{X}\right) & =\operatorname{det} R \Gamma\left(X,\left[\mathcal{E}_{X} \stackrel{\nabla}{\rightarrow} \mathcal{E}_{X} \otimes \Omega_{X}^{1}(\log D)\right]\right), \\
\lambda^{\prime}\left(\mathcal{E}_{X}\right) & =\operatorname{det} R \Gamma\left(X,\left[\mathcal{E}_{X} \stackrel{\nabla}{\rightarrow} \mathcal{E}_{X} \otimes \Omega_{X}\left(\log D^{\prime}\right)\right]\right)
\end{aligned}
$$

for short. Note that $\Gamma\left(\nabla: \mathcal{E}_{X}\right)=\Gamma^{\prime}\left(\nabla: \mathcal{E}_{X}\right)$ since $\operatorname{res}_{\infty} \nabla=0$. We define the isomorphisms. The isomorphism (1) is defined as in the text by the distinguished triangle

$$
\rightarrow R \Gamma\left(X, D R\left(\mathcal{E}_{X}\right)\right) \rightarrow R \Gamma\left(X, D R^{\prime}\left(\mathcal{E}_{X}\right)\right) \rightarrow \mathcal{E}_{X}(\infty)[-1] \rightarrow
$$

The isomorphism (2) follows from $\Omega_{X}^{1}(\log D)=\Omega_{X}^{1}\left(\log D^{\prime}\right) \otimes \mathcal{O}(-x)$. The commutativity is proved in the same way as in Lemma 4.2. Thus the proof of Theorem 3 for $X=\mathbb{P}_{k}^{1}$ is completed.

Now we prove Theorem 3 for an arbitrary proper smooth curve $X$. Let $f: X \rightarrow$ $Y=\mathbb{P}_{k}^{1}$ be a finite flat morphism. Let $W$ be an open subscheme of $Y$ such that on the inverse image $U=f^{*}(W)$ the restriction $\left.f\right|_{U}: U \rightarrow W$ is etale. We put $D=X-U$ and $E=Y-W$. We have a canonical isomorphism $f^{*} \Omega_{Y}^{1}(\log E) \simeq$ $\Omega_{X}^{1}(\log D)$. For an extension $\left(\mathcal{E}_{X}, \nabla\right)$ to $X$ of an integrable connection $(\mathcal{E}, \nabla)$ on $U$ of rank $r$, the canonical isomorphism $f_{*} D R_{X}\left(\mathcal{E}_{X}\right) \simeq D R_{Y}\left(f_{*} \mathcal{E}_{X}\right)$ induces an isomorphism

$$
\operatorname{det} R \Gamma\left(X, D R\left(\mathcal{E}_{X}\right)\right) \simeq \operatorname{det} R \Gamma\left(Y, D R\left(f_{*} \mathcal{E}_{X}\right)\right)
$$

The canonical isomorphisms

$$
N_{X / Y}\left(\operatorname{det} \mathcal{E}_{X} \otimes \mathcal{O}_{X}(-D)^{\otimes-r}\right) \simeq \operatorname{det} f_{*} \mathcal{E}_{X} \otimes \operatorname{det} f_{*} \mathcal{O}_{X}(-D)^{\otimes-r}
$$

and $f^{*} \Omega_{Y}^{1}(\log E) \simeq \Omega_{X}^{1}(\log D)$ induce an isomorphism

$$
\begin{aligned}
\left(\operatorname{det} \mathcal{E}_{X}(-r D), \Omega_{X}^{1}(\log D)\right) & \simeq\left(N_{X / Y} \operatorname{det} \mathcal{E}_{X}(-r D), \Omega_{Y}^{1}(\log E)\right) \\
& \simeq\left(\operatorname{det} f_{*} \mathcal{E}_{X} \otimes \operatorname{det} f_{*} \mathcal{O}_{X}(-D)^{\otimes-r}, \Omega_{Y}^{1}(\log E)\right) .
\end{aligned}
$$

They are compatible with the isomorphisms of Deligne-Riemann-Roch and the following diagram is commutative:

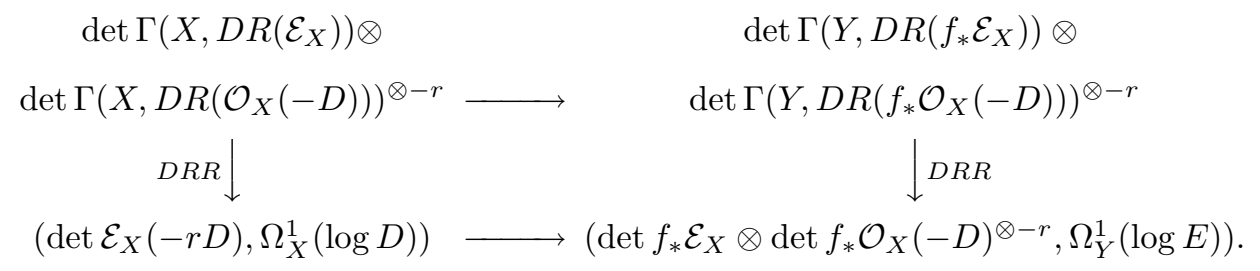

Let $\mathcal{M}=((\mathcal{E}, \nabla), V, \rho)$ be an object of $M_{k_{0}, F, X}(U)$ and $\left(\mathcal{E}_{X}, \nabla\right)$ be an extension of $(\mathcal{E}, \nabla)$ as above. Let $m_{\mathcal{E}_{X}}^{\nabla}=\left(\exp \left(\sum_{x \in D} \sigma\left(\operatorname{Tr}_{x / k}\left(\operatorname{Tr}\left(\operatorname{res}_{x} \nabla\right)\right)\right) \times \log m_{x}\right)\right)_{\sigma} \in$ $\left(k \otimes_{k_{i}} \mathbb{C}\right)^{\times}$where $m_{x}$ is the ramification index at $x$ and $\log m_{x} \in \mathbb{R}$ and let $\left[m_{\mathcal{E}_{X}}^{\nabla}\right]$ be the corresponding object of $P_{k_{0}, F}(k)$. For extensions $\mathcal{E}_{X} \subset \mathcal{E}_{X}^{\prime}$, the multiplication by the inverse of $\prod_{x \in D} m_{x}^{\operatorname{dim}_{k}\left(\mathcal{E}_{X, x}^{\prime} / \mathcal{E}_{X, x}\right)}$ on $k$ induces an isomorphism $\left[m_{\mathcal{E}_{X}}^{\nabla}\right] \simeq$ $\left[m_{\mathcal{E}_{X}^{\prime}}^{\nabla}\right]$. Define an object $\left[m_{\mathcal{M}}^{\nabla}\right]$ of $P_{k_{0}, F}(k)$ to be the projective limit of $\left[m_{\mathcal{E}_{X}}^{\nabla}\right]$ for small extensions $\mathcal{E}_{X}$. 
Claim 2. The canonical isomorphisms above induce isomorphisms

$$
\begin{gathered}
\operatorname{det}^{\Gamma} R \Gamma_{c}(U, \mathcal{M}) \otimes \operatorname{det}^{\Gamma} R \Gamma_{c}(U, 1)^{\otimes-r} \\
\simeq \operatorname{det}^{\Gamma} R \Gamma_{c}\left(W, f_{*} \mathcal{M}\right) \otimes \operatorname{det}^{\Gamma} R \Gamma_{c}\left(W, f_{*} 1\right)^{\otimes-r} \otimes\left[m_{\mathcal{M}}^{\nabla}\right], \\
\left(\operatorname{det} \mathcal{M}, \mathbf{c}_{X \bmod D}\right) \simeq\left(\operatorname{det} f_{*} \mathcal{M} \otimes\left(\operatorname{det} f_{*} \mathbf{1}\right)^{\otimes-r}, \mathbf{c}_{Y \bmod E}\right) \otimes\left[m_{\mathcal{M}}^{\nabla}\right] .
\end{gathered}
$$

Proof of Claim 2. The isomorphism (1) is proved in the same way as in Lemma 4.6 in Section 4. We show the isomorphism (2). We have

$$
\left(\Omega_{X}^{1}(\log D), \text { res }\right) \simeq f^{*}\left(\Omega_{Y}^{1}(\log E), \text { res }\right) \otimes\left(\mathcal{O}_{X},\left(m_{x} \times: \kappa(x) \rightarrow \kappa(x)\right)_{x \in D}\right) .
$$

Hence there is a canonical isomorphism

$$
\left(\operatorname{det} \mathcal{M}, \mathbf{c}_{X \bmod D}\right) \simeq\left(N_{X / Y} \operatorname{det} \mathcal{M}, \mathbf{c}_{Y \bmod E}\right) \otimes \bigotimes_{x \in D}\left(\operatorname{det} \mathcal{M}, m_{x}\right)_{x}^{\otimes-1} .
$$

By definition, there is a natural isomorphism $\bigotimes_{x \in D}\left(\operatorname{det} \mathcal{M}, m_{x}\right)_{x}^{\otimes-1} \simeq\left[m_{\mathcal{M}}^{\nabla}\right]$. Further by the canonical isomorphism $N_{X / Y} \operatorname{det} \mathcal{M} \simeq \operatorname{det} f_{*} \mathcal{M} \otimes \operatorname{det} f_{*} 1^{\otimes-r}$, the right hand sides of the above isomorphism and of the Claim are canonically isomorphic. It is easy to check that the de Rham component of the canonical isomorphism defined here is the same as that given there. Thus the Claim is proved.

We show that the Claim implies Theorem 3. Take a finite flat morphism $f: X \rightarrow$ $Y=\mathbb{P}_{k}^{1}$. Shrinking $U$ if necessary, we assume that $\left.f\right|_{U}$ is etale and $U=f^{*} f(U)$. By Theorem 3 for $Y=\mathbb{P}_{k}^{1}$ already proved, the isomorphism of Deligne-Riemann-Roch defines an isomorphism on the right hand sides of the isomorphisms in the Claim. Therefore by the commutativity above, the isomorphism of Deligne-Riemann-Roch also defines an isomorphism on the left hand sides of the isomorphisms. Thus Theorem 3 is proved.

\section{Appendix. Proof of Proposition 1}

We consider the spectral sequence

$$
E_{1}^{p, q}=\bigoplus_{x \in X^{p}} H_{x}^{p+q}\left(X, \mathcal{K}_{n}(X \bmod D)\right) \Rightarrow H^{p+q}\left(X, \mathcal{K}_{n}(X \bmod D)\right) .
$$

By the long exact sequence

$$
\rightarrow \bigoplus_{i} H_{x}^{r-1}\left(X, \mathcal{K}_{n}\left(D_{i}\right)\right) \rightarrow H_{x}^{r}\left(X, \mathcal{K}_{n}(X \bmod D)\right) \rightarrow H_{x}^{r}\left(X, \mathcal{K}_{n}(X)\right) \rightarrow
$$

and by the purity

$$
\begin{gathered}
H_{x}^{r}\left(X, \mathcal{K}_{n}(X)\right)= \begin{cases}K_{n-r}(x), & x \in X^{r}, \\
0 & \text { otherwise, }\end{cases} \\
H_{x}^{r-1}\left(X, \mathcal{K}_{n}\left(D_{i}\right)\right)= \begin{cases}K_{n-r+1}(x), & x \in X^{r} \cap D_{i}, \\
0 & \text { otherwise, }\end{cases}
\end{gathered}
$$

we have $E_{1}^{p, q}=0$ for $q \neq 0$ and an exact sequence

$$
0 \rightarrow \bigoplus_{x \in X^{p}}\left(\bigoplus_{i \in I_{x}} K_{n-p+1}(x)\right) \rightarrow E_{1}^{p, 0} \rightarrow \bigoplus_{x \in X^{p}} K_{n-p}(x) \rightarrow 0 .
$$


Therefore the spectral sequence degenerates at $E_{2}$-terms and we have an exact sequence

$$
\begin{aligned}
& \bigoplus_{y \in X_{1}} H_{y}^{n-1}\left(X, \mathcal{K}_{n}(X \bmod D)\right) \rightarrow \bigoplus_{x \in X_{0}} H_{x}^{n}\left(X, \mathcal{K}_{n}(X \bmod D)\right) \\
& \rightarrow C H^{n}(X \bmod D) \rightarrow 0 .
\end{aligned}
$$

Hence it is sufficient to define isomorphisms $\alpha_{x}: A_{x} \simeq H_{x}^{n}=H_{x}^{n}\left(X, \mathcal{K}_{n}(X \bmod D)\right)$ for $x \in X_{0}$ and $\beta_{y}: B_{y} \simeq H_{y}^{n-1}=H_{y}^{n-1}\left(X, \mathcal{K}_{n}(X \bmod D)\right)$ for $y \in X_{1}$ such that the diagram

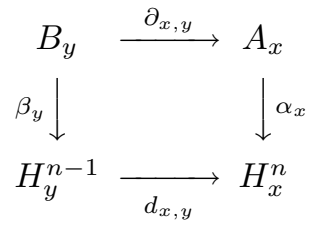

is commutative.

First we prove

Lemma A.1. Let $X$ be a smooth scheme over a field $k$ and $i: D \rightarrow X$ be the closed immersion of a smooth divisor. Then there is a canonical pairing

$$
R^{1} i^{!} \mathcal{K}_{p}(X \bmod D) \times \mathcal{K}_{q}(D) \rightarrow R^{1} i^{!} \mathcal{K}_{p+q}(X \bmod D)
$$

It is compatible with the natural pairing

$$
R^{1} i^{!} \mathcal{K}_{p}(X \bmod D) \times i^{*} \mathcal{K}_{q}(X) \rightarrow R^{1} i^{!} \mathcal{K}_{p+q}(X \bmod D)
$$

induced by the cup-product.

Proof. It is sufficient to define a functorial pairing

$$
H_{D \cap U}^{1}\left(U, \mathcal{K}_{p}(X \bmod D)\right) \times H^{0}\left(D \cap U, \mathcal{K}_{q}(D)\right) \rightarrow H_{D \cap U}^{1}\left(U, \mathcal{K}_{p+q}(X \bmod D)\right)
$$

for open subsets $U \subset X$ such that the diagram

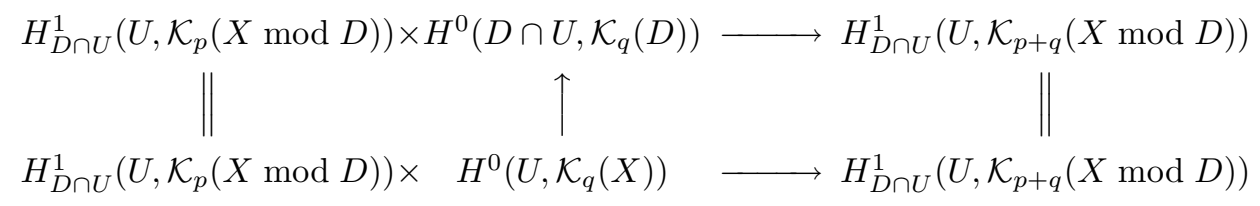

is commutative. Since it is easy to check the functoriality, we write $U=X$ for simplicity.

We consider the deformation to the normal bundle of $D$ in $X$. Let $\tilde{X}$ be the complement of the proper transform of $X \times\{0\}$ in the blowing-up of $\mathbb{A}_{X}^{1}$ at the center $D \times\{0\}$. Let $V=\mathbb{V}\left(N_{D / X}\right) \subset \tilde{X}$ be the exceptional divisor and $\tilde{D} \simeq \mathbb{A}_{D}^{1}$ be the proper transform of $\mathbb{A}_{D}^{1} \subset \mathbb{A}_{X}^{1}$. We obtain a commutative diagram

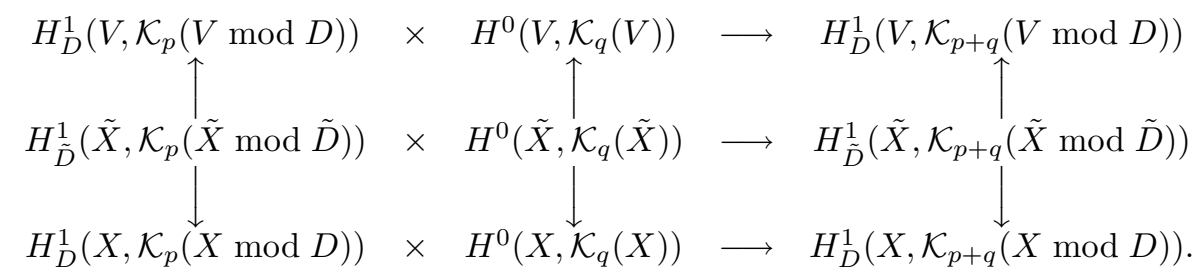


Here the upper vertical arrows are induced by the inclusion $V \rightarrow \tilde{X}$ at 0 and the lower ones are induced by $X \rightarrow \tilde{X}$ at 1 .

We show that the vertical arrows in the left and right columns are isomorphisms. By the purity of $K$-groups $R^{q} i{ }^{!} \mathcal{K}_{p}(X)=\mathcal{K}_{p-1}(D)$ for $q=1$ and $=0$ for otherwise, we have an exact sequence

$$
0 \rightarrow \mathcal{K}_{p}(D) \rightarrow R^{1} i^{!} \mathcal{K}_{p}(X \bmod D) \rightarrow \mathcal{K}_{p-1}(D) \rightarrow 0
$$

and $R^{q} i^{!} \mathcal{K}_{p}(X \bmod D)=0$ for $q \neq 1$. By the spectral sequence

$$
E_{2}^{p, q}=H^{p}\left(D, R^{q} i^{!} \mathcal{K}_{p}(X \bmod D)\right) \Rightarrow H_{D}^{p+q}\left(X, \mathcal{K}_{p}(X \bmod D)\right),
$$

we have an exact sequence

$$
\begin{aligned}
0 \rightarrow H^{0}\left(D, \mathcal{K}_{p}(D)\right) \rightarrow H_{D}^{1}\left(X, \mathcal{K}_{p}(X \bmod D)\right) & \rightarrow H^{0}\left(D, \mathcal{K}_{p-1}(D)\right) \\
& \rightarrow H^{1}\left(D, \mathcal{K}_{p}(D)\right) .
\end{aligned}
$$

We have similar exact sequences for $\tilde{D} \subset \tilde{X}$ and $D \subset V$. Now by the homotopy property of $K$-cohomology, the vertical arrows in the left and right columns are isomorphisms. Further by the homotopy property, the upper middle $H^{0}\left(V, \mathcal{K}_{q}(V)\right)$ is canonically isomorphic to $H^{0}\left(D, \mathcal{K}_{q}(D)\right)$. By these isomorphisms, the upper row gives the pairing

$$
H_{D}^{1}\left(X, \mathcal{K}_{p}(X \bmod D)\right) \times H^{0}\left(D, \mathcal{K}_{q}(D)\right) \rightarrow H_{D}^{1}\left(X, \mathcal{K}_{p+q}(X \bmod D)\right) .
$$

We check the compatibility. The lower arrow $H^{0}\left(\tilde{X}, \mathcal{K}_{q}(\tilde{X})\right) \rightarrow H^{0}\left(X, \mathcal{K}_{q}(X)\right)$ in the middle column is a surjection since it is induced by a section of the canonical map $\tilde{X} \rightarrow X$. Therefore it is sufficient to prove the commutativity of the diagram

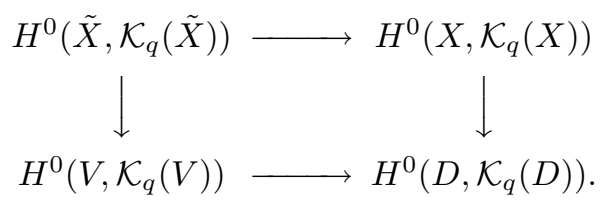

This follows from the commutative diagram

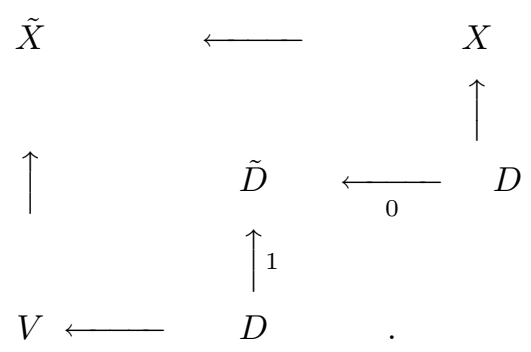

Here the arrows with tags 0 and 1 are the 0 -section and 1-section respectively and they induce the same isomorphisms $H^{0}\left(\tilde{D}, \mathcal{K}_{q}(\tilde{D})\right) \rightarrow H^{0}\left(D, \mathcal{K}_{q}(D)\right)$. Thus Lemma A.1 is proved.

We define the isomorphisms $\alpha_{x}$ and $\beta_{y}$ using Lemma A.1. First we consider $\alpha_{x}$ : $A_{x} \rightarrow H_{x}^{n}\left(X, \mathcal{K}_{n}(X \bmod D)\right)$ for $x \in X_{0}$. It is sufficient to define an isomorphism

$$
\alpha_{x, i}: A_{x, i} \rightarrow H_{x}^{n}\left(X, \mathcal{K}_{n}\left(X \bmod D_{i}\right)\right)=H_{x, i}^{n}
$$


such that the diagram

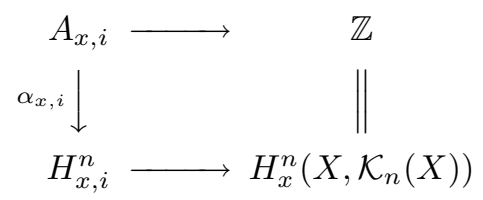

is commutative for each $i \in I_{x}$. Hence we may assume $D=D_{i}$ is irreducible and $x \in D$. By Lemma A.1, we obtain a pairing

$$
\begin{aligned}
H^{0}\left(D_{x}, R^{1} i^{!} \mathcal{K}_{1}(X \bmod D)\right) & \times H_{x}^{n-1}\left(D, \mathcal{K}_{n-1}(D)\right) \\
& \rightarrow H_{x}^{n-1}\left(D_{x}, R^{1} i^{!} \mathcal{K}_{n}(X \bmod D)\right)
\end{aligned}
$$

We show that

$$
\begin{gathered}
\Gamma\left(X_{x}-D_{x}, \mathbb{G}_{m}\right) /\left(1+\mathcal{I}_{x}\right) \simeq H^{0}\left(D_{x}, R^{1} i^{!} \mathcal{K}_{1}(X \bmod D)\right), \\
\mathbb{Z} \simeq H_{x}^{n-1}\left(D, \mathcal{K}_{n-1}(D)\right), \\
H_{x}^{n}\left(X_{x}, \mathcal{K}_{n}(X \bmod D)\right) \simeq H_{x}^{n-1}\left(D_{x}, R^{1} i^{!} \mathcal{K}_{n}(X \bmod D)\right)
\end{gathered}
$$

for $\mathcal{I}=\mathcal{O}(-D)$. Under the natural identification $A_{x} \simeq \Gamma\left(X_{x}-D_{x}, \mathbb{G}_{m}\right) / 1+m_{x}$, we also show that the pairing induces the required map $\alpha_{x}: A_{x} \rightarrow H_{x}^{n}\left(\mathcal{K}_{n}(X \bmod D)\right)$.

By purity, we have $H_{x}^{n-1}\left(D, \mathcal{K}_{n-1}(D)\right)=\mathbb{Z}$. Since $R^{q} i^{!} \mathcal{K}_{n}(X \bmod D)=0$ for $q \neq 1$, the spectral sequences

$$
\begin{gathered}
E_{2}^{p, q}=H^{p}\left(D_{x}, R^{q} i^{!} \mathcal{K}_{1}(X \bmod D)\right) \Rightarrow H_{D_{x}}^{p+q}\left(X \mathcal{K}_{x}, \mathcal{K}_{1}(X \bmod D)\right), \\
E_{2}^{p, q}=H_{x}^{p}\left(D_{x}, R^{q} i^{!} \mathcal{K}_{n}(X \bmod D)\right) \Rightarrow H_{x}^{p+q}\left(X, \mathcal{K}_{n}(X \bmod D)\right)
\end{gathered}
$$

degenerate at $E_{2}$ and we have isomorphisms

$$
\begin{aligned}
H^{0}\left(D_{x}, R^{1} i^{!} \mathcal{K}_{1}(X \bmod D)\right) & \simeq H_{D_{x}}^{1}\left(X_{x}, \mathcal{K}_{1}(X \bmod D)\right), \\
H_{x}^{n-1}\left(D_{x}, R^{1} i^{!} \mathcal{K}_{n}(X \bmod D)\right) & \simeq H_{x}^{n}\left(X, \mathcal{K}_{n}(X \bmod D)\right) .
\end{aligned}
$$

Further by the long exact sequence

$\rightarrow H^{p}\left(X_{x}, \mathcal{K}_{1}(X \bmod D)\right) \rightarrow H^{p}\left(X_{x}-D_{x}, \mathcal{K}_{1}(X)\right) \rightarrow H_{D_{x}}^{p+1}\left(X_{x}, \mathcal{K}_{1}(X \bmod D)\right) \rightarrow$ and by the equalities $H^{0}\left(X_{x}, \mathcal{K}_{1}(X \bmod D)\right)=1+\mathcal{I}_{x}$ for $\mathcal{I}=\mathcal{O}(-D)$, $H^{0}\left(X_{x}-D_{x}, \mathcal{K}_{1}(X)\right)=\Gamma\left(X_{x}-D_{x}, \mathbb{G}_{m}\right)$ and $H^{1}\left(X_{x}, \mathcal{K}_{1}(X \bmod D)\right)=0$, we have $H_{D_{x}}^{1}\left(X_{x}, \mathcal{K}_{1}(X \bmod D)\right)=\Gamma\left(X_{x}-D_{x}, \mathbb{G}_{m}\right) / 1+\mathcal{I}_{x}$. Hence the pairing above is a homomorphism $\tilde{\alpha}_{x}: \Gamma\left(X_{x}-D_{x}, \mathbb{G}_{m}\right) / 1+\mathcal{I}_{x} \rightarrow H_{x}^{n}\left(X, \mathcal{K}_{n}(X \bmod D)\right)$.

By the exact sequence

$$
0 \rightarrow \mathcal{K}_{q}(D) \rightarrow R^{1} i^{!} \mathcal{K}_{q}(X \bmod D) \rightarrow \mathcal{K}_{q-1}(D) \rightarrow 0
$$

the map $\tilde{\alpha}_{x}$ sits in the commutative diagram

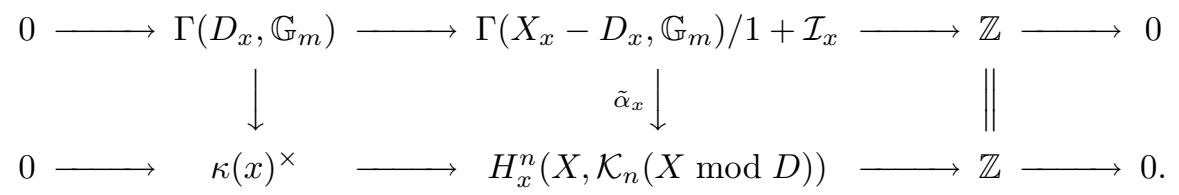

Therefore $\tilde{\alpha}_{x}$ annihilates $1+m_{x}$. Since $A_{x} \simeq \Gamma\left(X_{x}-D_{x}, \mathbb{G}_{m}\right) / 1+m_{x}$, the map $\tilde{\alpha}_{x}$ induces the required isomorphism $\alpha_{x}: A_{x} \rightarrow H_{x}^{n}$. 
Next we consider $\beta_{y}$ for $y \in X_{1}$. Similarly as above, it is sufficient to define an isomorphism $\beta_{y}: B_{y} \rightarrow H_{y}^{n-1}\left(X, \mathcal{K}_{n}(X \bmod D)\right)$ assuming $D$ is irreducible and $y \in D$ such that the diagram

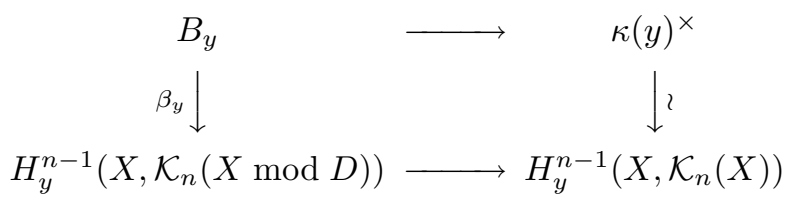

is commutative. By Lemma A.1, we obtain a pairing

$$
\begin{aligned}
H^{0}\left(D_{y}, R^{1} i^{!} \mathcal{K}_{1}(X \bmod D)\right) & \times H_{y}^{n-2}\left(D, \mathcal{K}_{n-1}(D)\right) \\
& \rightarrow H_{y}^{n-2}\left(D_{y}, R^{1} i^{!} \mathcal{K}_{n}(X \bmod D)\right) .
\end{aligned}
$$

Similarly as above, we have isomorphisms

$$
\begin{gathered}
H^{0}\left(D_{y}, R^{1} i^{!} \mathcal{K}_{1}(X \bmod D)\right)=\Gamma\left(X_{y}-D_{y}, \mathbb{G}_{m}\right) / 1+\mathcal{I}_{y}, \\
H_{y}^{n-2}\left(D, \mathcal{K}_{n-1}(D)\right)=\kappa(y)^{\times}
\end{gathered}
$$

and

$$
H_{y}^{n-2}\left(D_{y}, R^{1} i^{!} \mathcal{K}_{n}(X \bmod D)\right)=H_{y}^{n-1}\left(X, \mathcal{K}_{n}(X \bmod D)\right)
$$

The same argument as above shows that the pairing induces a morphism $\tilde{\beta}_{y}$ : $A_{y} \otimes \kappa(y)^{\times} \rightarrow H_{y}^{n-1}\left(X, \mathcal{K}_{n}(X \bmod D)\right)$ and further an isomorphism $\beta_{y}: B_{y} \rightarrow$ $H_{y}^{n-1}\left(X, \mathcal{K}_{n}(X \bmod D)\right)$.

Finally we show that the boundary map $d=d_{1}^{n-1,0}$ of the spectral sequence coincides with $\partial: \bigoplus B_{y} \rightarrow \bigoplus A_{x}$ under the identification by $\alpha$ and $\beta$. It is sufficient to show that the $(x, y)$-components $d_{x, y}$ and $\partial_{x, y}$ are the same for each $x \in X_{0}$ and $y \in X_{1}$. If $x$ is not in the closure $Y$ of $\{y\}$, we have $d_{x, y}=\partial_{x, y}=0$. Hence we assume $x \in Y$. If $x \notin D$, then $y \notin D$ and $d_{x, y}=\partial_{x, y}=\operatorname{ord}_{x}: \kappa(y)^{\times} \rightarrow \mathbb{Z}$. Hence we assume $x \in D$. By considering each $i$-th component for $i \in I_{x}$, we may assume $D$ is irreducible. First we check the case where $Y$ meets $D$ transversally at $x$. Then by Lemma A.1, we obtain a commutative diagram

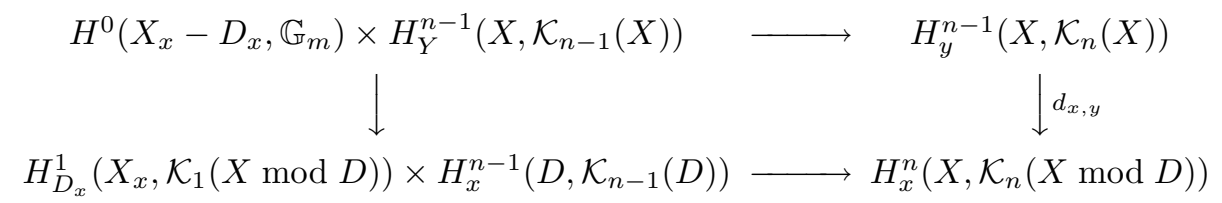

since

$$
\begin{aligned}
H_{D_{x}}^{1}\left(X_{x}, \mathcal{K}_{1}(X \bmod D)\right) & =H^{0}\left(D_{x}, R^{1} i^{!} \mathcal{K}_{1}(X \bmod D)\right), \\
H_{x}^{n}\left(X, \mathcal{K}_{n}(X \bmod D)\right) & =H_{x}^{n-1}\left(D_{x}, R^{1} i^{!} \mathcal{K}_{n}(X \bmod D)\right) .
\end{aligned}
$$

Hence the diagram

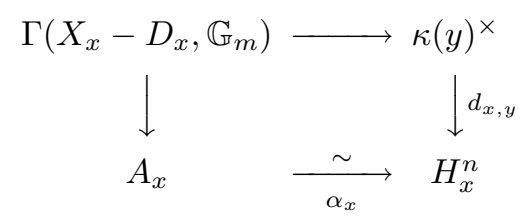


is commutative. Since the diagram

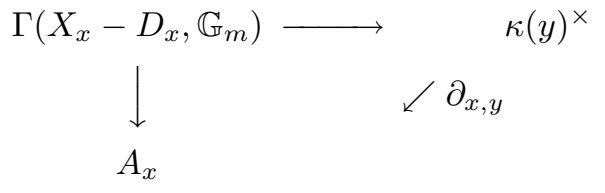

is commutative by definition and since $\Gamma\left(X_{x}-D_{x}, \mathbb{G}_{m}\right) \rightarrow \kappa(y)^{\times}$is surjective, the differentials $d_{x, y}$ and $\partial_{x, y}$ are the same.

We consider the general case. First we show that the problem is etale local. Let $\varphi: X^{\prime} \rightarrow X$ be an etale morphism $\varphi\left(x^{\prime}\right)=x$ and $\eta_{x^{\prime}}=\left\{y^{\prime} \in X^{\prime} ; \varphi\left(y^{\prime}\right)=\right.$ $y$ and $\left.\overline{\left\{y^{\prime}\right\}} \ni x^{\prime}\right\}$. Then we have commutative diagrams

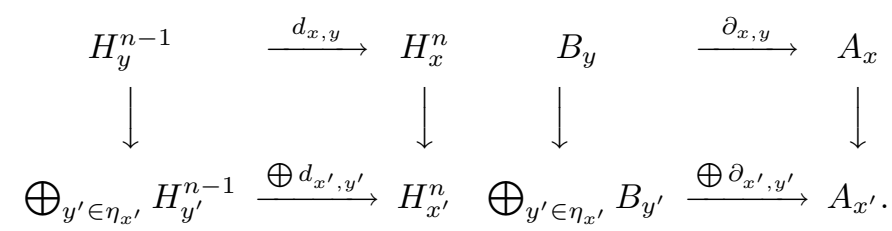

Since $A_{x} \rightarrow A_{x^{\prime}}$ is injective, to prove $d_{x, y}=\partial_{x, y}$, it is enough to show $d_{x^{\prime}, y^{\prime}}=\partial_{x^{\prime}, y^{\prime}}$ for $y^{\prime} \in \eta_{x^{\prime}}$.

Claim 1. By taking an etale neighborhood, we find closed integral subschemes $W \subset$ $Z \subset X$ satisfying the following conditions:

(1) $x \in W$ and $y \in Z$.

(2) $W$ is regular of dimension 1 and meets transversally with $D$ at $x$.

(3) $Z$ is of dimension 2. $Z-W$ is regular and meets transversally with $D$.

(4) The normalization $\tilde{Z}$ is regular and the inverse image $\tilde{T}$ of $D$ in $\tilde{Z}$ is regular.

(5) The reduced inverse image $\tilde{W}$ of $W$ in $\tilde{Z}$ is regular and $\tilde{W}$ meets transversally with $\tilde{T}$.

(6) $\tilde{T} \cap \tilde{W}=($ inverse image of $x$ in $\tilde{Z})$ contains only one point $\tilde{x}$.

Proof of Claim 1. By localizing if necessary, we may take a smooth projection $\pi$ : $X \rightarrow D$ such that the immersion $D \rightarrow X$ is a section. Since it is trivial if $\pi(y)=x$, we assume $\pi(Y)=T$ is a curve. By further localizing if necessary, we may assume that $T-\{x\}$ is normal and that the inverse image of $x$ in the normalization $\tilde{T}$ of $T$ consists of only one point $\tilde{x}$. Then $W=\pi^{*}(x)$ and $Z=\pi^{*}(T)$ satisfy the conditions.

Changing the notation, we use the same characters $X, Z, W$ etc. in Claim 1 for their inverse images in the localization $X_{x}$ at $x$. We consider the canonical morphism

$$
d: H_{Z-x}^{n-1}\left(X, \mathcal{K}_{n}(X \bmod D)\right) \rightarrow H_{x}^{n}\left(X, \mathcal{K}_{n}(X \bmod D)\right) .
$$

Let $\zeta$ be the generic point of $Z$ and $Z^{*}=Z-x$. By the spectral sequence $E_{1}^{p, q}=$ $\bigoplus_{z \in X^{p} \cap Z^{*}} H_{z}^{p+q}\left(X, \mathcal{K}_{n}(X \bmod D)\right) \Rightarrow H_{Z^{*}}^{p+q}\left(X, \mathcal{K}_{n}(X \bmod D)\right)$ degenerating at $E_{2}$-terms, we have an exact sequence

$$
K_{2}(\kappa(\zeta)) \rightarrow \bigoplus_{z \in Z_{1}} H_{z}^{n-1}\left(X, \mathcal{K}_{n}(X \bmod D)\right) \rightarrow H_{Z^{*}}^{n-1}\left(X, \mathcal{K}_{n}(X \bmod D)\right) \rightarrow 0
$$

To complete the proof of Proposition 1, it is sufficient to prove the following 
Claim 2. (1) By identifying $\beta: \bigoplus_{z \in Z_{1}} B_{z} \rightarrow \bigoplus_{z \in Z_{1}} H_{z}^{n-1}\left(X, \mathcal{K}_{n}(X \bmod D)\right)$, the map $\bigoplus \partial_{x, z}: \bigoplus_{z \in Z_{1}} B_{z} \rightarrow A_{x}$ annihilates the image of $K_{2}(\kappa(\zeta)) \rightarrow$ $\bigoplus_{z \in Z_{1}} H_{z}^{n-1}\left(X, \mathcal{K}_{n}(X \bmod D)\right)$.

(2) For the generic point $w \in Z_{1}$ of $W$, the canonical map

$$
H_{w}^{n-1}\left(X, \mathcal{K}_{n}(X \bmod D)\right) \rightarrow H_{Z^{*}}^{n-1}\left(X, \mathcal{K}_{n}(X \bmod D)\right)
$$

is surjective.

We show that Claim 2 proves Proposition 1. In fact Claim 2 (1) implies $\bigoplus \partial_{x, z}$ induces a map $\partial: H_{Z^{*}}^{n-1}\left(X, \mathcal{K}_{n}(X \bmod D)\right) \rightarrow A_{x}$. By identifying $\alpha_{x}: A_{x} \rightarrow$ $H_{x}^{n}\left(X, \mathcal{K}_{n}(X \bmod D)\right)$, we see $d=\partial$ on the image of $H_{w}^{n-1}\left(X, \mathcal{K}_{n}(X \bmod D)\right)$ since $W$ meets $D$ transversally and we have $d_{x, w}=\partial_{x, w}$. By Claim $2(2)$, we have $d=\partial$ on the whole $H_{Z^{*}}^{n-1}\left(X, \mathcal{K}_{n}(X \bmod D)\right)$. This proves $d_{x, z}=\partial_{x, z}$ for all $z \in Z_{1}$, in particular for $y$.

Proof of Claim 2. (1) We take an arbitrary element $a \in K_{2}(\zeta)$ and compute its image by

$$
K_{2}(\zeta) \rightarrow \bigoplus_{z \in Z_{1}} B_{z} \rightarrow A_{x}
$$

By the condition (6) in Claim 1, the intersection $T=D \cap Z$ is integral. Let $t$ be the generic point of $T$. For $z \in Z_{1}$, we have $B_{z}=\kappa(z)^{\times}$for $z \neq t$. We take a prime element $\pi \in \mathcal{O}_{X, x}$ of $D$. Then it defines splittings $A_{x}=\mathbb{Z} \oplus \kappa(x)^{\times}$and $B_{t}=\kappa(t)^{\times} \oplus K_{2}(t)$. For $z \in Z_{1}, \neq t$, let $a_{z}=\partial_{z, \zeta}(a) \in \kappa(z)^{\times}$and for $z=t$, let $\left(a_{t}^{1}, a_{t}^{2}\right)=\partial_{t, \zeta}(a) \in \kappa(t)^{\times} \oplus K_{2}(t)=B_{t}$. The first component $a_{t}^{1} \in \kappa(t)^{\times}$is the usual tame symbol of $a \in K_{2}(\zeta)$. Then by definition of $\partial$, we have

$$
\partial_{x, z} \circ \partial_{z, \zeta}(a)= \begin{cases}\left(\operatorname{ord}_{x}\left(a_{z}\right),(-1)^{\operatorname{ord}_{x}\left(a_{z}\right)}\left(\pi, a_{z}\right)_{x}\right), & z \neq t, \\ \left(\operatorname{ord}_{x}\left(a_{t}^{1}\right), \partial a_{t}^{2}\right), & z=t .\end{cases}
$$

The second component $\partial a_{t}^{2} \in \kappa(x)^{\times}$is the usual tame symbol of $a_{t}^{2} \in K_{2}(t)$. Therefore it is sufficient to prove

$$
\begin{array}{r}
\sum_{z \in Z_{1}, \neq t} \operatorname{ord}_{x}\left(a_{z}\right)+\operatorname{ord}_{x}\left(a_{t}^{1}\right)=0 \\
\prod_{z \in Z_{1}, \neq t}(-1)^{\operatorname{ord}_{x}\left(a_{z}\right)}\left(\pi, a_{z}\right)_{x} \times \partial\left(a_{t}^{2}\right)=1 .
\end{array}
$$

The first equality is the usual reciprocity law for $a \in K_{2}(\zeta)$. By the first one, the second is equivalent to

$$
\prod_{z \in Z_{1}, \neq t}\left(\pi, a_{z}\right)_{x} \times(-1)^{\operatorname{ord}_{x}\left(a_{t}^{1}\right)} \partial\left(a_{t}^{2}\right)=1 .
$$

To prove this, we use the reciprocity law for $\{\pi, a\} \in K_{3}(\zeta)$. Since $\partial_{z, \zeta}(\{\pi, a\})=$ $\left\{\pi, a_{z}\right\} \in K_{2}(z)$ for $z \neq t$, it is sufficient to show that

$$
\partial_{x, t} \circ \partial_{t, \zeta}(\{\pi, a\})=(-1)^{\operatorname{ord}_{x}\left(a_{t}^{1}\right)} \partial a_{t}^{2}
$$

in $\kappa(x)^{\times}$. We prove this by showing

$$
\partial_{t, \zeta}(\{\pi, a\})=\left\{-1, a_{t}^{1}\right\}+a_{t}^{2}
$$


in $K_{2}(t)$. Let $\tilde{a}_{1} \in \Gamma\left(X_{t}, \mathbb{G}_{m}\right)$ be a lifting of $a_{t}^{1} \in \kappa(t)^{\times}$. Then $\tilde{a}_{2}=a-\left\{\pi, \tilde{a}_{1}\right\} \in$ $K_{2}(\zeta)$ is in the kernel of the tame symbol $K_{2}(\zeta) \rightarrow \kappa(t)^{\times}$and hence in $K_{2}\left(\mathcal{O}_{Z, t}\right)$. By the commutativity of the diagram

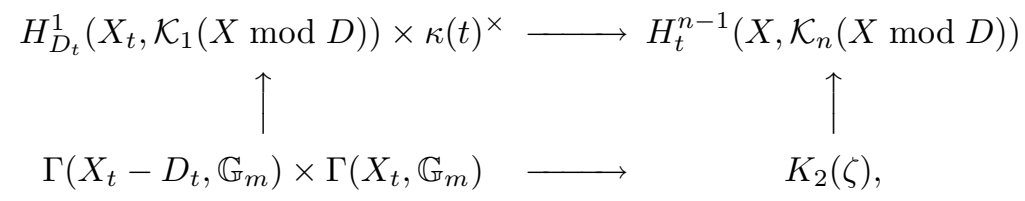

we have $\partial_{t, \zeta}\left(\left\{\pi, \tilde{a}_{1}\right\}\right)=\left(a_{t}^{1}, 0\right)$. By the commutative diagram

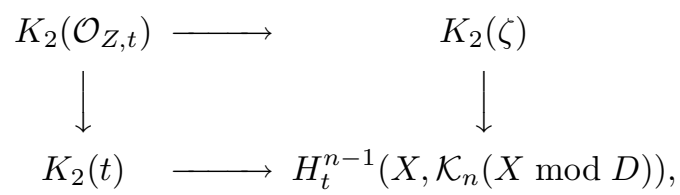

$a_{t}^{2}$ is the reduction of $\tilde{a}_{2}$. Now we have

$$
\{\pi, a\}=\left\{\pi, \pi, \tilde{a}_{1}\right\}+\left\{\pi, \tilde{a}_{2}\right\}=\left\{\pi,\left(\left\{-1, \tilde{a}_{1}\right\}+\tilde{a}_{2}\right)\right\} .
$$

Therefore we have

$$
\partial_{t, \zeta}(\{\pi, a\})=\left\{-1, a_{t}^{1}\right\}+a_{t}^{2} .
$$

Thus the assertion (1) is proved.

(2) By the exact sequence

$$
\begin{aligned}
H_{w}^{n-1}\left(X, \mathcal{K}_{n}(X \bmod D)\right) & \rightarrow H_{Z-x}^{n-1}\left(X, \mathcal{K}_{n}(X \bmod D)\right) \\
& \rightarrow H_{Z-W}^{n-1}\left(X, \mathcal{K}_{n}(X \bmod D)\right)
\end{aligned}
$$

and by the purity

$$
H_{Z-W}^{n-1}\left(X, \mathcal{K}_{n}(X \bmod D)\right) \simeq H^{1}\left(Z-W, \mathcal{K}_{2}(Z-W \bmod T-x)\right),
$$

it is sufficient to show that $H^{1}\left(Z-W, \mathcal{K}_{2}(Z-W \bmod T-x)\right)=0$. By the exact sequence

$$
\begin{aligned}
H^{1}\left(\tilde{Z}, \mathcal{K}_{2}(\tilde{Z} \bmod \tilde{T})\right) & \rightarrow H^{1}\left(Z-W, \mathcal{K}_{2}(Z-W \bmod T-x)\right) \\
& \rightarrow H_{\tilde{W}}^{2}\left(\tilde{Z}, \mathcal{K}_{2}(\tilde{Z} \bmod \tilde{T})\right)
\end{aligned}
$$

and by the purity

$$
H_{\tilde{W}}^{2}\left(\tilde{Z}, \mathcal{K}_{2}(\tilde{Z} \bmod \tilde{T})\right) \simeq H^{1}\left(\tilde{W}, \mathcal{K}_{1}(\tilde{W} \bmod \tilde{x})\right),
$$

it is reduced to showing that

$$
\begin{aligned}
H^{1}\left(\tilde{Z}, \mathcal{K}_{2}(\tilde{Z} \bmod \tilde{T})\right) & =\operatorname{Coker}\left(K_{2}\left(\mathcal{O}_{\tilde{Z}}\right) \rightarrow\left(K_{2}\left(\mathcal{O}_{\tilde{T}}\right)\right)\right)=0, \\
H^{1}\left(\tilde{W}, \mathcal{K}_{1}(\tilde{W} \bmod \tilde{x})\right) & =\operatorname{Coker}\left(\mathcal{O}_{\tilde{W}}^{\times}\right) \rightarrow \kappa(\tilde{x})^{\times}=0 .
\end{aligned}
$$

Since $K_{2}\left(\mathcal{O}_{\tilde{T}}\right)$ is generated by symbols, the maps are surjective. Thus Claim 2 and hence Proposition 1 are proved. 


\section{REFERENCES}

[A] G.Anderson, Cyclotomy and a covering of the Taniyama group, Compositio Math. 57 (1985), 153-217. MR 88e:11045

[D1] P.Deligne, Equations différentielles à points singuliers réguliers, LNM 163, Springer - Verlag, Berlin-Heidelberg-New York, 1970. MR 54:5232

[D2] Théorie de Hodge II, Publ. Math. IHES 40 (1973), 5-58. MR 58:16653a

[D3] L La formule de dualité globale, SGA 4 Exp. XVIII, LNM, vol. 305, Springer-Verlag, Berlin-Heidelberg-New York, 1973, pp. 481-587.

[D4] Valeurs de fonctions L et périodes d'integrales, Proceedings of Symposia in Pure Math., vol. 33 Part 2, Amer. Math. Soc., 1979, pp. 313-346. MR 81d:12009

[D5] — La conjecture de Weil II, Publ. Math. IHES 52 (1981), 313-428. MR 83c:14017

[D6] Le groupe fondamental de la droite projective moin trois points, Galois groups over $\mathbb{Q}$, Springer-Verlag, Berlin-Heidelberg-New York, 1986, pp. 79-297. MR 90m:14016

[D7] Le déterminant de la cohomologie, Contemporary Math., vol. 67, Amer. Math. Soc., Providence, RI, 1987, pp. 93-177. MR 89b:32038

[G] H.Gillet, Gersten's conjecture for the K-theory with torsion coefficients of a discrete valuation ring, Journal of algebra 103 (1986), 377-380. MR 87 m:18017

[K-M] F.Knudsen-D.Mumford, The projectivity of the moduli space of stable curves I, preliminaries on "det" and "Div", Math. Scand. 39-1 (1976), 19-55. MR 55:10465

[O] C.Okonek-M.Schneider-H.Spindler, Vector bundles on complex projective spaces., Progress in Math., vol. 3, Birkheauser, Boston, Mass., 1980. MR 81b:14001

[Q] D.Quillen, Higher algebraic K-theory I, LNM 341, Springer-Verlag, Berlin - Heidelberg New York, 1972, pp. 85-147. MR 49:2895

[Sa] N.Saavedra, Catégorie tannakienne, LNM, vol. 265, Springer-Verlag, Berlin-HeidelbergNew York, 1972.

[S1] T.Saito, $\epsilon$-factor of tamely ramified sheaf on a variety, Inventiones Math. 113 (1993), 389-417. MR 94m:14025

[S2] _ Jacobi sum Hecke character, de Rham discriminant and determinant of $\ell$-adic cohomologies, Journal of algebraic geometry 30 (1994), 411-434. MR 95f:14036

[ST] T.Saito, T.Terasoma, A determinant formula for period integrals, Proc. Japan academy Ser. A 69 (1993), 131-135. MR 94m:14009

[T] T.Terasoma, A product formula for period integrals, Math. Ann. 298 (1994), 577-589. MR 95b:32035

Department of Mathematical Sciences, University of Tokyo, 3-8-1 Komaba Meguro, 153 TOKYO, JAPAN

E-mail address: t-saito@ms.u-tokyo.ac.jp

E-mail address: terasoma@ms.u-tokyo.ac.jp 